\title{
Hydrosilylation-Promoted Furan Diels-Alder Cycloadditions with Stereoselectivity Controlled by the Silyl Group
}

\author{
Zhi-Yun Liu ${ }^{\dagger}$, Ming Zhang ${ }^{\dagger}$, Xiao-Chen Wang* \\ State Key Laboratory and Institute of Elemento-Organic Chemistry, College of Chemistry, \\ Nankai University, Tianjin 300071, China
}

\section{Table of Contents}

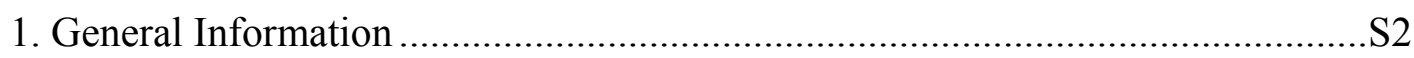

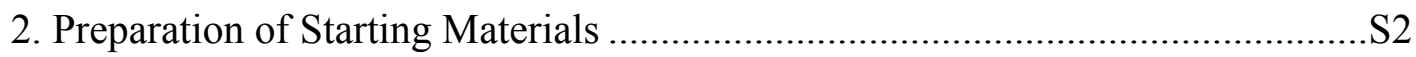

3. Optimization of the Reaction Conditions......................................................S8

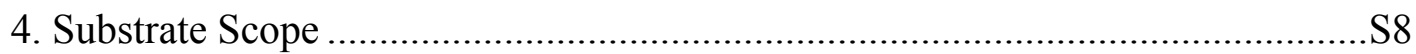

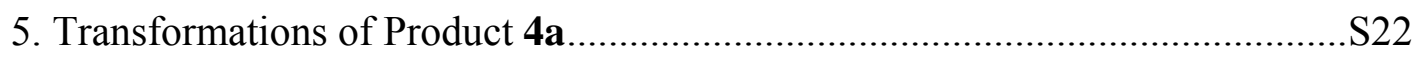

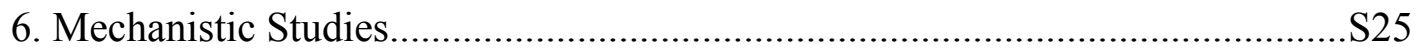

6.1 Deuterium-Labelling Experiments.................................................S25

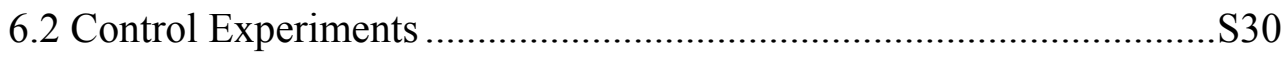

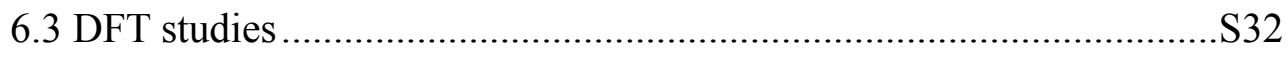

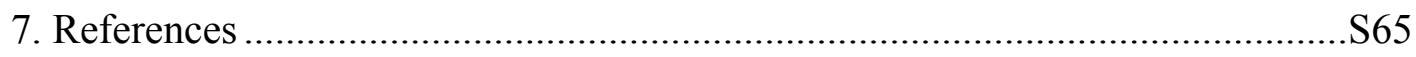

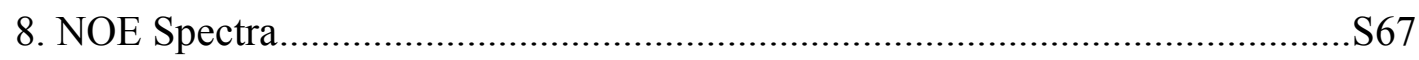

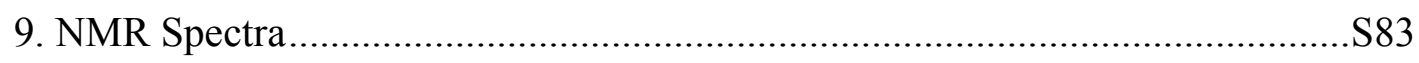




\section{General Information}

Unless otherwise noted, all materials were used as received from commercial sources without further purification. Dry solvents were obtained by distillation from drying reagents according to procedures described in Purification of Laboratory Chemicals (5th Edition) written by Wilfred L.F. Armarego and Christina L.L. Chai (Elsevier, 2003). Analytical thin layer chromatography (TLC) was performed on Huanghai precoated ( $0.25 \mathrm{~mm}$ thickness) silica gel plates with F254 indicator. Visualization was accomplished with UV light $(254 \mathrm{~nm})$ or the potassium permanganate stain solution. Flash chromatography was performed with silica gel $(32-63 \mu \mathrm{m})$ supplied by Sanpont Group. ${ }^{1} \mathrm{H}$ NMR spectra were recorded on a Bruker DRX-400 (400 MHz) spectrometer and chemical shifts were reported in ppm. The peak information was described as: $\mathrm{br}=$ broad, $\mathrm{s}=$ singlet, $\mathrm{d}=$ doublet, $\mathrm{t}=$ triplet, $\mathrm{q}=$ quartet, $\mathrm{m}=$ multiplet; coupling constants in Hz. ${ }^{13} \mathrm{C}$ NMR spectra were recorded on a Bruker DRX-400 (101 $\mathrm{MHz})$ spectrometer with complete proton decoupling. Chemical shifts of the NMR spectra were calibrated by the literature values of the solvent residual peaks. High-resolution mass spectra (HRMS) were obtained on a Varian 7.0T FTMS (ESI or MALDI $). B\left(\mathrm{C}_{6} \mathrm{~F}_{5}\right)_{3}$ was prepared according to the previous literature. ${ }^{[1]}$

\section{Preparation of Starting Materials}

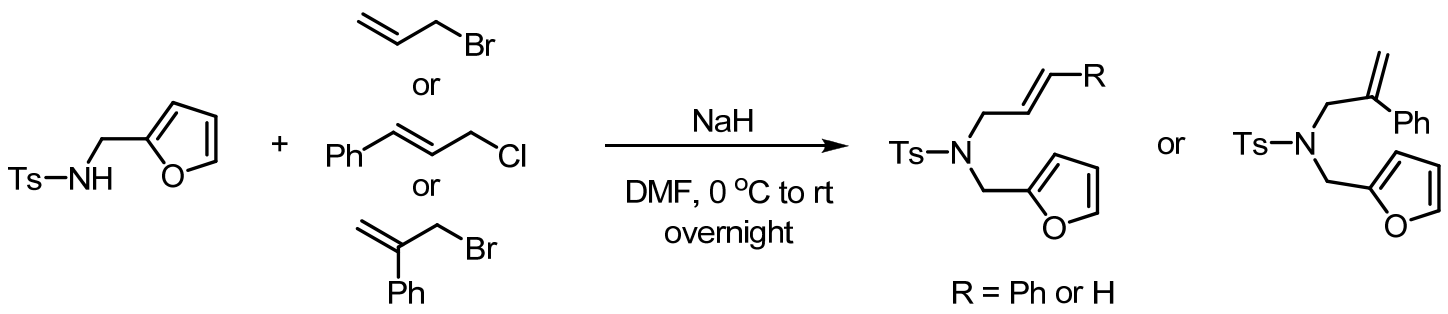

General procedure A: The tosylated furfurylamine ${ }^{[2]}$ (10 mmol, 1 equiv) was added to a solution of $\mathrm{NaH}$ ( $60 \%$ in mineral oil, $15 \mathrm{mmol}, 1.5$ equiv) in dry DMF (30 mL). The solution was stirred under argon at room temperature for $1 \mathrm{~h}$, followed by the addition of the corresponding allylic halide (12 mmol, 1.2 equiv). The resulting mixture was stirred at room temperature for $20 \mathrm{~h}$, and then was poured into a mixture of ice and water $(60 \mathrm{~mL})$. The mixture was extracted with dichloromethane $(3 \times 30$ $\mathrm{mL}$ ). The combined organic layers were dried over $\mathrm{MgSO}_{4}$, filtered and concentrated in vacuo. The residue was subjected to column chromatography on silica gel (PE/EA $=20: 1$ as eluent) to give the corresponding product. Substrates $1 \mathbf{g}, \mathbf{1 h}$ and $\mathbf{1 k}$ were prepared using this procedure.

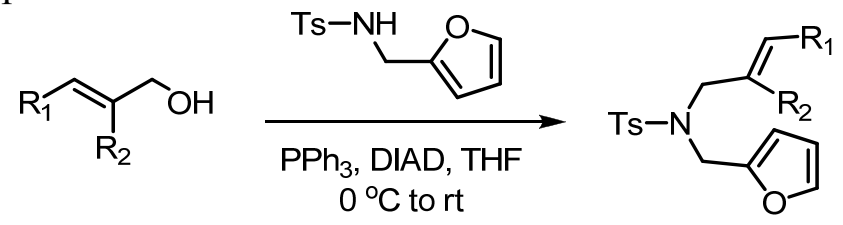


General procedure B: The corresponding allylic alcohol ( $7.2 \mathrm{mmol}, 1.2$ equiv) and $\mathrm{PPh}_{3}$ (8.4 mmol, 1.4 equiv) were added to a solution of the tosylated furfurylamine (6 mmol, 1 equiv) in dry THF $(20 \mathrm{~mL})$ under argon. Diisopropyl azodicarboxylate $(8.4$ mmol, 1.4 equiv) was then added dropwise at $0{ }^{\circ} \mathrm{C}$, and the resulting mixture was stirred at room temperature for $5 \mathrm{~h}$. The mixture was concentrated in vacuo. The residue was subjected to column chromatography on silica gel (PE/EA $=20: 1$ as eluent) to give the corresponding product. Substrates 1a, 1b, 1c, 1d, 1e, 1f, 1i, 1j, 1l, 1m, 1n, 1o, 1p, 1q, 1r were prepared using this procedure. The allylic alcohol precursors for preparation of $\mathbf{1} \mathbf{a}^{[3]}, \mathbf{1} \mathbf{c}^{[4]}, \mathbf{1 d}^{[5]}, \mathbf{1 e}^{[6]}, \mathbf{1} \mathbf{j}^{[7]}, \mathbf{1} \mathbf{m}^{[8]}, \mathbf{1 n}^{[8]}$ and $\mathbf{1} \mathbf{p}^{[8]}$ had been synthesized and characterized in the previous literatures. The allylic alcohol precursors for preparation of $\mathbf{1 l}$ was prepared according to the previous literature ${ }^{[9]}$, The allylic alcohol precursors for preparation of $\mathbf{1 b}, \mathbf{1 f}, \mathbf{1 i}, \mathbf{1 0}, \mathbf{1 q}$ and $\mathbf{1 r}$ were purchased from commercial sources.

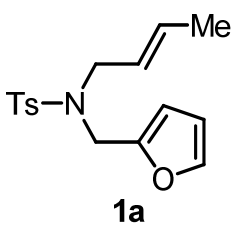

(E)- $N$-(But-2-en-1-yl)- $N$-(furan-2-ylmethyl)-4-methylbenzenesulfonamide (1a). Colorless oil. ${ }^{1} \mathrm{H}$ NMR (400 MHz, $\left.\mathrm{CDCl}_{3}\right) \delta 7.62(\mathrm{~d}, J=8.3 \mathrm{~Hz}, 2 \mathrm{H}), \quad 7.25-7.20$ (m, $3 \mathrm{H}), 6.23(\mathrm{dd}, J=3.1,1.9 \mathrm{~Hz}, 1 \mathrm{H}), 6.11(\mathrm{~d}, J=3.1 \mathrm{~Hz}, 1 \mathrm{H}), 5.62-5.51(\mathrm{~m}, 1 \mathrm{H})$, 5.29-5.20 (m, 1H), $4.36(\mathrm{~s}, 2 \mathrm{H}), 3.69(\mathrm{~d}, J=6.7 \mathrm{~Hz}, 2 \mathrm{H}), 2.38(\mathrm{~s}, 3 \mathrm{H}), 1.62(\mathrm{dd}, J=6.5$, $1.1 \mathrm{~Hz}, 3 \mathrm{H}) .{ }^{13} \mathrm{C}$ NMR $\left(101 \mathrm{MHz}, \mathrm{CDCl}_{3}\right) \delta 149.9,143.1,142.3,137.3,131.0,129.5$, 127.2, 125.1, 110.3, 109.3, 49.0, 42.4, 21.5, 17.7. HRMS (ESI) calcd. for $\mathrm{C}_{16} \mathrm{H}_{20} \mathrm{NO}_{3} \mathrm{~S}^{+}$ $(\mathrm{M}+\mathrm{H})^{+}:$306.1158, Found: 306.1158 .

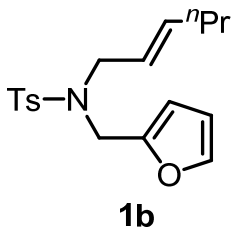

(E)- $N$-(Furan-2-ylmethyl)- $N$-(hex-2-en-1-yl)-4-methylbenzenesulfonamide (1b). Colorless oil. ${ }^{1} \mathrm{H}$ NMR $\left(400 \mathrm{MHz}, \mathrm{CDCl}_{3}\right) \delta 7.65(\mathrm{~d}, J=8.2 \mathrm{~Hz}, 2 \mathrm{H}), 7.27-7.22(\mathrm{~m}$, $3 \mathrm{H}), 6.25(\mathrm{dd}, J=3.0,1.9 \mathrm{~Hz}, 1 \mathrm{H}), 6.13(\mathrm{~d}, J=3.0 \mathrm{~Hz}, 1 \mathrm{H}), 5.56(\mathrm{dt}, J=13.8,6.7 \mathrm{~Hz}$, $1 \mathrm{H}), 5.32-5.19(\mathrm{~m}, 1 \mathrm{H}), 4.38(\mathrm{~s}, 2 \mathrm{H}), 3.72(\mathrm{~d}, J=6.7 \mathrm{~Hz}, 2 \mathrm{H}), 2.41(\mathrm{~s}, 3 \mathrm{H}), 1.95(\mathrm{q}, J$ $=7.0 \mathrm{~Hz}, 2 \mathrm{H}), 1.43-1.25(\mathrm{~m}, 2 \mathrm{H}), 0.86(\mathrm{t}, J=7.4 \mathrm{~Hz}, 3 \mathrm{H}) .{ }^{13} \mathrm{C}$ NMR $(101 \mathrm{MHz}$, $\left.\mathrm{CDCl}_{3}\right) \delta 150.1,143.1,142.4,137.5,136.4,129.6,127.4,124.0,110.4,109.4,49.1$, 42.4, 34.3, 22.3, 21.6, 13.7. HRMS (ESI) calcd. for $\mathrm{C}_{18} \mathrm{H}_{24} \mathrm{NO}_{3} \mathrm{~S}^{+}(\mathrm{M}+\mathrm{H})^{+}$: 334.1471 , Found: 334.1472.

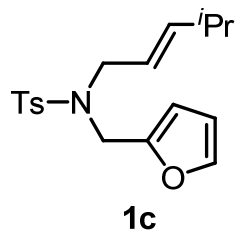


(E)- $N$-(Furan-2-ylmethyl)-4-methyl- $N$-(4-methylpent-2-en-1-yl)benzenesulfonami de (1c). Colorless oil. ${ }^{1} \mathrm{H}$ NMR $\left(400 \mathrm{MHz}, \mathrm{CDCl}_{3}\right) \delta 7.65(\mathrm{~d}, J=8.2 \mathrm{~Hz}, 2 \mathrm{H})$, 7.28-7.21 (m, 3H), $6.25(\mathrm{dd}, J=3.1,1.9 \mathrm{~Hz}, 1 \mathrm{H}), 6.13(\mathrm{~d}, J=3.1 \mathrm{~Hz}, 1 \mathrm{H}), 5.56-5.46(\mathrm{~m}$, $1 \mathrm{H}), 5.23-5.12(\mathrm{~m}, 1 \mathrm{H}), 4.37(\mathrm{~s}, 2 \mathrm{H}), 3.72(\mathrm{~d}, J=6.7 \mathrm{~Hz}, 2 \mathrm{H}), 2.41(\mathrm{~s}, 3 \mathrm{H}), 2.32-2.13$ (m, 1H), 0.94-0.88 (m, 6H). $\left.{ }^{13} \mathrm{C} \mathrm{NMR} \mathrm{(101} \mathrm{MHz,} \mathrm{CDCl}_{3}\right) \delta 150.0,143.4,143.1,142.4$, 137.5, 129.6, 127.3, 120.9, 110.4, 109.4, 49.2, 42.5, 30.8, 22.2, 21.6. HRMS (ESI) calcd. for $\mathrm{C}_{18} \mathrm{H}_{24} \mathrm{NO}_{3} \mathrm{~S}^{+}(\mathrm{M}+\mathrm{H})^{+}: 334.1471$, Found: 334.1469 .<smiles>[13CH3]N(C/C=C\Br)Cc1ccco1</smiles>

$\mathbf{N}$-(Furan-2-ylmethyl)-4-methyl- $\mathbf{N}$-(4-phenylbut-2-en-1-yl)benzenesulfonamide (1d). Colorless oil. $E / Z=8.5: 1 .{ }^{1} \mathrm{H} \mathrm{NMR}\left(400 \mathrm{MHz}, \mathrm{CDCl}_{3}\right) \delta(E): 7.63(\mathrm{~d}, J=8.2 \mathrm{~Hz}$, 2H), 7.31-7.16 (m, 6H), 7.10 (d, $J=7.2 \mathrm{~Hz}, 2 \mathrm{H}), 6.24-6.20(\mathrm{~m}, 0.89 \mathrm{H}), 6.11-6.09$ (m, $0.89 \mathrm{H}), 5.78-5.64(\mathrm{~m}, 1 \mathrm{H}), 5.39-5.28(\mathrm{~m}, 1 \mathrm{H}), 4.37(\mathrm{~s}, 1.79 \mathrm{H}), 3.75(\mathrm{~d}, J=6.7 \mathrm{~Hz}$, $1.79 \mathrm{H}), 3.30(\mathrm{~d}, J=6.7 \mathrm{~Hz}, 2 \mathrm{H}), 2.39(\mathrm{~s}, 3 \mathrm{H}) .(\mathrm{Z})$ (distinguishable peaks): 6.26-6.24 (m, $0.11 \mathrm{H}), 6.15-6.12(\mathrm{~m}, 0.11 \mathrm{H}), 4.41(\mathrm{~s}, 0.21 \mathrm{H}), 3.94(\mathrm{~d}, J=7.0 \mathrm{~Hz}, 0.21 \mathrm{H}) .{ }^{13} \mathrm{C} \mathrm{NMR}$ (101 MHz, $\mathrm{CDCl}_{3}$, signals of the dominant $E$ isomer) $\delta 149.8,143.1,142.4,139.7$, 137.3, 134.7, 129.5, 128.6, 128.5, 127.3, 126.2, 125.4, 110.3, 109.4, 48.9, 42.7, 38.7, 21.6. HRMS (ESI) calcd. for $\mathrm{C}_{22} \mathrm{H}_{24} \mathrm{NO}_{3} \mathrm{~S}^{+}(\mathrm{M}+\mathrm{H})^{+}: 382.1471$, Found: 382.1470 .<smiles>[B]N(C/C=C\CCBr)Cc1ccco1</smiles>

(E)- $\boldsymbol{N}$-(5-Bromopent-2-en-1-yl)- $\boldsymbol{N}$-(furan-2-ylmethyl)-4-methylbenzenesulfonami de (1e). White solid. ${ }^{1} \mathrm{H}$ NMR (400 MHz, $\left.\mathrm{CDCl}_{3}\right) \delta 7.66(\mathrm{~d}, J=8.3 \mathrm{~Hz}, 2 \mathrm{H}), 7.30-7.23$ (m, 3H), 6.29-6.24 (m, 1H), $6.17(\mathrm{~d}, J=2.9 \mathrm{~Hz}, 1 \mathrm{H}), 5.61-5.48(\mathrm{~m}, 1 \mathrm{H}), 5.42-5.34(\mathrm{~m}$, $1 \mathrm{H}), 4.40(\mathrm{~s}, 2 \mathrm{H}), 3.74(\mathrm{~d}, J=6.5 \mathrm{~Hz}, 2 \mathrm{H}), 3.34(\mathrm{t}, J=6.8 \mathrm{~Hz}, 2 \mathrm{H}), 2.54$ (q, $J=6.5 \mathrm{~Hz}$, 2H), $2.42(\mathrm{~s}, 3 \mathrm{H}) .{ }^{13} \mathrm{C} \mathrm{NMR}\left(101 \mathrm{MHz}, \mathrm{CDCl}_{3}\right) \delta 149.8,143.3,142.5,137.3,132.2$, 129.6, 127.4, 127.3, 110.4, 109.6, 48.8, 42.7, 35.3, 32.2, 21.6. HRMS (ESI) calcd. for $\mathrm{C}_{17} \mathrm{H}_{21} \mathrm{BrNO}_{3} \mathrm{~S}^{+}(\mathrm{M}+\mathrm{H})^{+}:$398.0420, Found: 398.0422 .<smiles>C=C(C)CN([As])Cc1ccco1</smiles>

$1 f$

$\boldsymbol{N}$-(Furan-2-ylmethyl)-4-methyl- $\boldsymbol{N}$-(2-methylallyl)benzenesulfonamide

(1). Colorless oil. ${ }^{1} \mathrm{H}$ NMR $\left(400 \mathrm{MHz}, \mathrm{CDCl}_{3}\right) \delta 7.62(\mathrm{~d}, J=8.2 \mathrm{~Hz}, 2 \mathrm{H}), 7.26-7.16(\mathrm{~m}$, $3 \mathrm{H}), 6.24-6.17(\mathrm{~m}, 1 \mathrm{H}), 6.08(\mathrm{~d}, J=3.2 \mathrm{~Hz}, 1 \mathrm{H}), 4.91(\mathrm{~d}, J=17.3 \mathrm{~Hz}, 2 \mathrm{H}), 4.36$ (s, 2H), $3.70(\mathrm{~s}, 2 \mathrm{H}), 2.39(\mathrm{~s}, 3 \mathrm{H}), 1.69(\mathrm{~s}, 3 \mathrm{H}) .{ }^{13} \mathrm{C} \mathrm{NMR}\left(101 \mathrm{MHz}, \mathrm{CDCl}_{3}\right) \delta 149.7$, 143.0, 142.3, 139.9, 137.2, 129.4, 127.3, 114.8, 110.2, 109.7, 53.2, 42.4, 21.5, 19.7. HRMS (ESI) calcd. for $\mathrm{C}_{16} \mathrm{H}_{20} \mathrm{NO}_{3} \mathrm{~S}^{+}(\mathrm{M}+\mathrm{H})^{+}$: 306.1158, Found: 306.1157. 


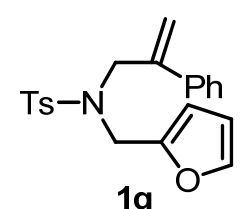

$\mathbf{N}$-(Furan-2-ylmethyl)-4-methyl- $\boldsymbol{N}$-(2-phenylallyl)benzenesulfonamide (1g). White solid. ${ }^{1} \mathrm{H}$ NMR $\left(400 \mathrm{MHz}, \mathrm{CDCl}_{3}\right) \delta 7.59(\mathrm{~d}, J=8.3 \mathrm{~Hz}, 2 \mathrm{H}), 7.42-7.35(\mathrm{~m}, 2 \mathrm{H})$, 7.34-7.28 (m, 3H), $7.22(\mathrm{~d}, J=8.1 \mathrm{~Hz}, 2 \mathrm{H}), 7.18(\mathrm{~d}, J=1.0 \mathrm{~Hz}, 1 \mathrm{H}), 6.21(\mathrm{dd}, J=3.1$, $1.9 \mathrm{~Hz}, 1 \mathrm{H}), 6.05(\mathrm{~d}, J=3.1 \mathrm{~Hz}, 1 \mathrm{H}), 5.48(\mathrm{~s}, 1 \mathrm{H}), 5.28(\mathrm{~d}, J=0.7 \mathrm{~Hz}, 1 \mathrm{H}), 4.35(\mathrm{~s}, 2 \mathrm{H})$, 4.25 (s, 2H), 2.41 (s, 3H). $\left.{ }^{13} \mathrm{C} \mathrm{NMR} \mathrm{(101} \mathrm{MHz,} \mathrm{CDCl}_{3}\right) \delta 149.7,143.2,142.5,142.4$, 138.6, 136.9, 129.5, 128.5, 128.1, 127.5, 126.6, 116.5, 110.4, 109.8, 50.8, 42.7, 21.6. HRMS (ESI) calcd. for $\mathrm{C}_{21} \mathrm{H}_{22} \mathrm{NO}_{3} \mathrm{~S}^{+}(\mathrm{M}+\mathrm{H})^{+}: 368.1315$, Found: 368.1313 .

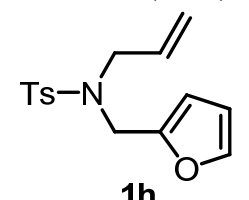

$\mathrm{N}$-Allyl- $\mathrm{N}$-(furan-2-ylmethyl)-4-methylbenzenesulfonamide (1h). Colorless oil. ${ }^{1} \mathrm{H}$ NMR $\left(400 \mathrm{MHz}, \mathrm{CDCl}_{3}\right) \delta 7.66(\mathrm{~d}, J=8.3 \mathrm{~Hz}, 2 \mathrm{H}), 7.28-7.23(\mathrm{~m}, 3 \mathrm{H}), 6.25(\mathrm{dd}, J=$ $3.1,1.9 \mathrm{~Hz}, 1 \mathrm{H}), 6.14(\mathrm{~d}, J=3.1 \mathrm{~Hz}, 1 \mathrm{H}), 5.70-5.54(\mathrm{~m}, 1 \mathrm{H}), 5.21-5.16(\mathrm{~m}, 1 \mathrm{H})$, 5.16-5.12 (m, 1H), 4.39 (s, 2H), $3.77(\mathrm{~d}, J=6.3 \mathrm{~Hz}, 2 \mathrm{H}), 2.40(\mathrm{~s}, 3 \mathrm{H}) .{ }^{13} \mathrm{C}$ NMR $(101$ $\left.\mathrm{MHz}, \mathrm{CDCl}_{3}\right) \delta 149.7,143.2,142.4,137.2,132.5,129.6,127.2,119.2,110.3,109.5$, 49.6, 42.7, 21.5. HRMS (ESI) calcd. for $\mathrm{C}_{15} \mathrm{H}_{18} \mathrm{NO}_{3} \mathrm{~S}^{+}(\mathrm{M}+\mathrm{H})^{+}:$292.1002, Found: 292.1000. This compound was characterized by previous literature ${ }^{[10]}$.

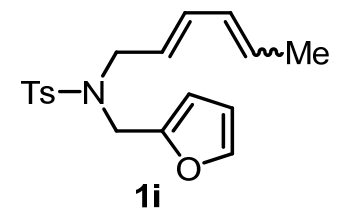

$\boldsymbol{N}$-(Furan-2-ylmethyl)- $\boldsymbol{N}$-((2E)-hexa-2,4-dien-1-yl)-4-methylbenzenesulfonamide (1i). Colorless oil. $E / Z=5.1: 1 .{ }^{1} \mathrm{H} \mathrm{NMR}\left(400 \mathrm{MHz}, \mathrm{CDCl}_{3}\right) \delta(E): 7.65(\mathrm{~d}, J=7.1 \mathrm{~Hz}$, 2H), 7.28-7.21 (m, 3H), 6.31-6.21 (m, 1H), 6.17-6.12 (m, 1H), 6.10-5.90 (m, 1.85H), $5.72-5.61(\mathrm{~m}, 0.84 \mathrm{H}), 5.35-5.26(\mathrm{~m}, 0.84 \mathrm{H}), 4.38(\mathrm{~s}, 2 \mathrm{H}), 3.78(\mathrm{~d}, J=6.8 \mathrm{~Hz}, 1.67 \mathrm{H})$, $2.41(\mathrm{~s}, 3 \mathrm{H}), 1.74(\mathrm{~d}, J=6.7 \mathrm{~Hz}, 3 \mathrm{H})$. (Z) (distinguishable peaks): 5.54-5.37 (m, $0.33 \mathrm{H}), 5.18-5.02(\mathrm{~m}, 0.14 \mathrm{H}), 3.83(\mathrm{~d}, J=6.9 \mathrm{~Hz}, 0.33 \mathrm{H}) .{ }^{13} \mathrm{C}$ NMR $(101 \mathrm{MHz}$, $\mathrm{CDCl}_{3}$, signals of the dominant $E$ isomer) $\delta 149.9,143.2,142.5,137.4,135.1,130.6$, 130.5, 129.6, 127.4, 124.1, 110.4, 109.5, 48.9, 42.5, 21.6, 18.2. HRMS (ESI) calcd. for $\mathrm{C}_{18} \mathrm{H}_{22} \mathrm{NO}_{3} \mathrm{~S}^{+}(\mathrm{M}+\mathrm{H})^{+}: 332.1315$, Found: 332.1314 .

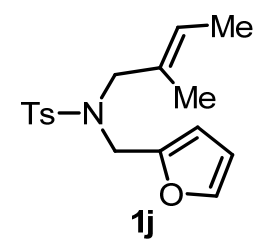

(E)- $N$-(Furan-2-ylmethyl)-4-methyl- $N$-(2-methylbut-2-en-1-yl)benzenesulfonami de (1j). Colorless oil. ${ }^{1} \mathrm{H}$ NMR $\left(400 \mathrm{MHz}, \mathrm{CDCl}_{3}\right) \delta 7.59(\mathrm{~d}, J=8.2 \mathrm{~Hz}, 2 \mathrm{H}), 7.21$ (d, $J=8.2 \mathrm{~Hz}, 2 \mathrm{H}), 7.18-7.13(\mathrm{~m}, 1 \mathrm{H}), 6.20(\mathrm{dd}, J=3.0,1.8 \mathrm{~Hz}, 1 \mathrm{H}), 6.04(\mathrm{~d}, J=3.0 \mathrm{~Hz}$, $1 \mathrm{H}), 5.39(\mathrm{q}, J=6.6 \mathrm{~Hz}, 1 \mathrm{H}), 4.31(\mathrm{~s}, 2 \mathrm{H}), 3.66(\mathrm{~s}, 2 \mathrm{H}), 2.38(\mathrm{~s}, 3 \mathrm{H}), 1.59(\mathrm{~d}, J=6.6$ 
$\mathrm{Hz}, 3 \mathrm{H}), 1.55(\mathrm{~s}, 3 \mathrm{H}) .{ }^{13} \mathrm{C} \mathrm{NMR}\left(101 \mathrm{MHz}, \mathrm{CDCl}_{3}\right) \delta 150.1,142.9,142.2,137.3$, 130.4, 129.4, 127.3, 124.7, 110.2, 109.4, 55.3, 42.1, 21.6, 13.6. HRMS (ESI) calcd. for $\mathrm{C}_{17} \mathrm{H}_{22} \mathrm{NO}_{3} \mathrm{~S}^{+}(\mathrm{M}+\mathrm{H})^{+}: 320.1315$, Found: 320.1312.<smiles>[As]N(C/C=C\c1ccccc1)Cc1ccccc1</smiles>

$\boldsymbol{N}$-Cinnamyl- $\boldsymbol{N}$-(furan-2-ylmethyl)-4-methylbenzenesulfonamide (1k). White solid. ${ }^{1} \mathrm{H}$ NMR (400 MHz, $\left.\mathrm{CDCl}_{3}\right) \delta 7.68(\mathrm{~d}, J=8.3 \mathrm{~Hz}, 2 \mathrm{H}), 7.35-7.18(\mathrm{~m}, 8 \mathrm{H}), 6.43(\mathrm{~d}, J$ $=15.9 \mathrm{~Hz}, 1 \mathrm{H}), 6.26(\mathrm{dd}, J=3.1,1.9 \mathrm{~Hz}, 1 \mathrm{H}), 6.17(\mathrm{~d}, J=3.1 \mathrm{~Hz}, 1 \mathrm{H}), 5.96(\mathrm{dt}, J=$ 15.9, $6.7 \mathrm{~Hz}, 1 \mathrm{H}), 4.43(\mathrm{~s}, 2 \mathrm{H}), 3.93(\mathrm{~d}, J=6.7 \mathrm{~Hz}, 2 \mathrm{H}), 2.41(\mathrm{~s}, 3 \mathrm{H}) .{ }^{13} \mathrm{C}$ NMR $(101$ $\left.\mathrm{MHz}_{2} \mathrm{CDCl}_{3}\right) \delta 149.9,143.3,142.6,137.3,136.3,134.3,129.7,128.7,128.0,127.4$, 126.6, 123.7, 110.5, 109.6, 49.3, 42.9, 21.6. HRMS (ESI) calcd. for $\mathrm{C}_{21} \mathrm{H}_{22} \mathrm{NO}_{3} \mathrm{~S}^{+}$ $(\mathrm{M}+\mathrm{H})^{+}:$368.1315, Found: 368.1316 .

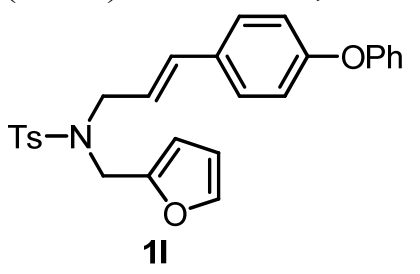

(E)- $N$-(Furan-2-ylmethyl)-4-methyl- $N$-(3-(4-phenoxyphenyl)allyl)benzenesulfona mide (1l). Colorless oil. ${ }^{1} \mathrm{H}$ NMR (400 MHz, $\left.\mathrm{CDCl}_{3}\right) \delta 7.68(\mathrm{~d}, J=8.2 \mathrm{~Hz}, 2 \mathrm{H}), 7.33$ (t, $J=8.0 \mathrm{~Hz}, 2 \mathrm{H}), 7.28-7.21(\mathrm{~m}, 5 \mathrm{H}), 7.11(\mathrm{t}, J=7.4 \mathrm{~Hz}, 1 \mathrm{H}), 7.00(\mathrm{~d}, J=7.7 \mathrm{~Hz}, 2 \mathrm{H})$, $6.93(\mathrm{~d}, J=8.6 \mathrm{~Hz}, 2 \mathrm{H}), 6.40(\mathrm{~d}, J=15.8 \mathrm{~Hz}, 1 \mathrm{H}), 6.26(\mathrm{dd}, J=3.0,1.9 \mathrm{~Hz}, 1 \mathrm{H}), 6.16$ $(\mathrm{d}, J=3.0 \mathrm{~Hz}, 1 \mathrm{H}), 5.87(\mathrm{dt}, J=15.8,6.8 \mathrm{~Hz}, 1 \mathrm{H}), 4.43(\mathrm{~s}, 2 \mathrm{H}), 3.92(\mathrm{~d}, J=6.8 \mathrm{~Hz}, 2 \mathrm{H})$, $2.40(\mathrm{~s}, 3 \mathrm{H}) .{ }^{13} \mathrm{C} \mathrm{NMR}\left(101 \mathrm{MHz}, \mathrm{CDCl}_{3}\right) \delta 157.2,157.0,149.9,143.3,142.6,137.3$, 133.5, 131.5, 129.9, 129.6, 127.9, 127.4, 123.6, 122.7, 119.1, 118.9, 110.4, 109.6, 49.4, 42.9, 21.6. HRMS (ESI) calcd. for $\mathrm{C}_{27} \mathrm{H}_{25} \mathrm{NO}_{4} \mathrm{SNa}^{+}(\mathrm{M}+\mathrm{Na})^{+}: 482.1397$, Found: 482.1394 .

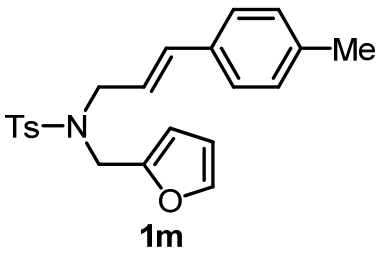

(E)- $\boldsymbol{N}$-(Furan-2-ylmethyl)-4-methyl- $\boldsymbol{N}$-(3-(p-tolyl)allyl)benzenesulfonamide (1m). White solid. ${ }^{1} \mathrm{H}$ NMR (400 MHz, $\left.\mathrm{CDCl}_{3}\right) \delta 7.68(\mathrm{~d}, J=8.2 \mathrm{~Hz}, 2 \mathrm{H}), 7.29-7.23(\mathrm{~m}, 3 \mathrm{H})$, $7.17(\mathrm{~d}, J=8.1 \mathrm{~Hz}, 2 \mathrm{H}), 7.11(\mathrm{~d}, J=8.1 \mathrm{~Hz}, 2 \mathrm{H}), 6.40(\mathrm{~d}, J=15.8 \mathrm{~Hz}, 1 \mathrm{H}), 6.26(\mathrm{dd}, J$ $=3.1,1.9 \mathrm{~Hz}, 1 \mathrm{H}), 6.16(\mathrm{~d}, J=3.1 \mathrm{~Hz}, 1 \mathrm{H}), 5.90(\mathrm{dt}, J=15.8,6.8 \mathrm{~Hz}, 1 \mathrm{H}), 4.43(\mathrm{~s}, 2 \mathrm{H})$, $3.92(\mathrm{~d}, J=6.8 \mathrm{~Hz}, 2 \mathrm{H}), 2.42(\mathrm{~s}, 3 \mathrm{H}), 2.33(\mathrm{~s}, 3 \mathrm{H}) .{ }^{13} \mathrm{C} \mathrm{NMR}\left(101 \mathrm{MHz}, \mathrm{CDCl}_{3}\right) \delta$ $149.9,143.3,142.6,137.9,137.4,134.3,133.5,129.6,129.4,127.4,126.5,122.6$, 110.4, 109.6, 49.4, 42.8, 21.6, 21.3. HRMS (ESI) calcd. for $\mathrm{C}_{22} \mathrm{H}_{24} \mathrm{NO}_{3} \mathrm{~S}^{+}(\mathrm{M}+\mathrm{H})^{+}$: 382.1471, Found: 382.1473. 


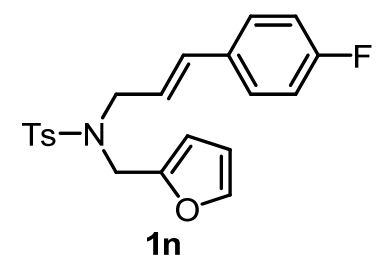

(E)- $N$-(3-(4-Fluorophenyl)allyl)- $N$-(furan-2-ylmethyl)-4-methylbenzenesulfonami de (1n). White solid. ${ }^{1} \mathrm{H}$ NMR $\left(400 \mathrm{MHz}, \mathrm{CDCl}_{3}\right) \delta 7.68(\mathrm{~d}, J=8.3 \mathrm{~Hz}, 2 \mathrm{H})$, 7.30-7.18 (m, 5H), 7.03-6.93 (m, 2H), 6.39 (d, $J=15.8 \mathrm{~Hz}, 1 \mathrm{H}), 6.28-6.24(\mathrm{~m}, 1 \mathrm{H})$, $6.16(\mathrm{~d}, J=3.0 \mathrm{~Hz}, 1 \mathrm{H}), 5.88(\mathrm{dt}, J=15.8,6.7 \mathrm{~Hz}, 1 \mathrm{H}), 4.42(\mathrm{~s}, 2 \mathrm{H}), 3.92(\mathrm{~d}, J=6.7$ $\mathrm{Hz}, 2 \mathrm{H}), 2.41(\mathrm{~s}, 3 \mathrm{H}) .{ }^{13} \mathrm{C} \mathrm{NMR}\left(101 \mathrm{MHz}, \mathrm{CDCl}_{3}\right) \delta 162.5(\mathrm{~d}, J=247.3 \mathrm{~Hz}), 149.8$, 143.3, 142.6, 137.2, 132.9, $132.5(\mathrm{~d}, J=3.1 \mathrm{~Hz}), 129.6,128.1$ (d, $J=8.0 \mathrm{~Hz}), 127.4$, $123.6(\mathrm{~d}, J=1.9 \mathrm{~Hz}), 115.5(\mathrm{~d}, J=21.6 \mathrm{~Hz}), 110.4,109.6,49.3,43.0,21.6$. HRMS (ESI) calcd. for $\mathrm{C}_{21} \mathrm{H}_{21} \mathrm{FNO}_{3} \mathrm{~S}^{+}(\mathrm{M}+\mathrm{H})^{+}$: 386.1221 , Found: 386.1222 .

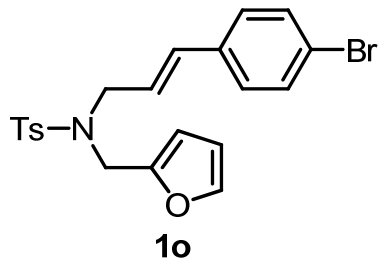

(E)- $N$-(3-(4-Bromophenyl)allyl)- $\boldsymbol{N}$-(furan-2-ylmethyl)-4-methylbenzenesulfonami de (1o). White solid. ${ }^{1} \mathrm{H}$ NMR (400 MHz, $\left.\mathrm{CDCl}_{3}\right) \delta 7.67$ (d, J=8.1 Hz, 2H), 7.39 (d, $J$ $=8.3 \mathrm{~Hz}, 2 \mathrm{H}), 7.33-7.19(\mathrm{~m}, 3 \mathrm{H}), 7.11(\mathrm{~d}, J=8.3 \mathrm{~Hz}, 2 \mathrm{H}), 6.35(\mathrm{~d}, J=15.9 \mathrm{~Hz}, 1 \mathrm{H})$, $6.29-6.20(\mathrm{~m}, 1 \mathrm{H}), 6.18-6.12(\mathrm{~m}, 1 \mathrm{H}), 5.95(\mathrm{dt}, J=15.7,6.6 \mathrm{~Hz}, 1 \mathrm{H}), 4.41(\mathrm{~s}, 2 \mathrm{H}), 3.91$ $(\mathrm{d}, J=6.6 \mathrm{~Hz}, 2 \mathrm{H}), 2.39$ (s, 3H). ${ }^{13} \mathrm{C} \mathrm{NMR}\left(101 \mathrm{MHz}, \mathrm{CDCl}_{3}\right) \delta 149.7,143.3,142.6$, 137.1, 135.2, 132.7, 131.6, 129.6, 128.0, 127.3, 124.7, 121.6, 110.4, 109.6, 49.2, 43.1, 21.5. HRMS (ESI) calcd. for $\mathrm{C}_{21} \mathrm{H}_{21} \mathrm{BrNO}_{3} \mathrm{~S}^{+}(\mathrm{M}+\mathrm{H})^{+}$: 446.0420 , Found: 446.0418 .

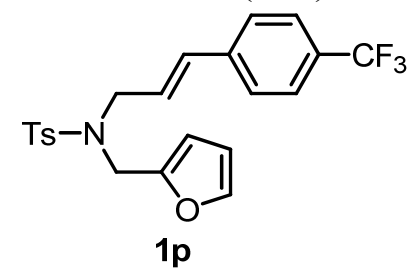

(E)- $N$-(Furan-2-ylmethyl)-4-methyl- $N$-(3-(4-(trifluoromethyl)phenyl)allyl)benzen esulfonamide (1p). White solid. ${ }^{1} \mathrm{H}$ NMR $\left(400 \mathrm{MHz}, \mathrm{CDCl}_{3}\right) \delta 7.69(\mathrm{~d}, J=8.0 \mathrm{~Hz}, 2 \mathrm{H})$, $7.55(\mathrm{~d}, J=8.0 \mathrm{~Hz}, 2 \mathrm{H}), 7.36(\mathrm{~d}, J=8.0 \mathrm{~Hz}, 2 \mathrm{H}), 7.29-7.25(\mathrm{~m}, 3 \mathrm{H}), 6.46(\mathrm{~d}, J=15.9$ $\mathrm{Hz}, 1 \mathrm{H}), 6.28-6.24(\mathrm{~m}, 1 \mathrm{H}), 6.20-6.15(\mathrm{~m}, 1 \mathrm{H}), 6.13-6.01(\mathrm{~m}, 1 \mathrm{H}), 4.43(\mathrm{~s}, 2 \mathrm{H}), 3.95(\mathrm{~d}$, $J=6.4 \mathrm{~Hz}, 2 \mathrm{H}), 2.41(\mathrm{~s}, 3 \mathrm{H}) .{ }^{13} \mathrm{C} \mathrm{NMR}\left(101 \mathrm{MHz}, \mathrm{CDCl}_{3}\right) \delta 149.7,143.5,142.7,139.9$, $137.2,132.5,129.7$ (q, $J=32.5 \mathrm{~Hz}), 129.7,127.5,126.9,126.7,125.6$ (q, $J=3.8 \mathrm{~Hz})$, $124.2(\mathrm{q}, J=271.9 \mathrm{~Hz}), 110.6,109.8,49.3,43.4,21.6$. HRMS (ESI) calcd. for $\mathrm{C}_{22} \mathrm{H}_{21} \mathrm{~F}_{3} \mathrm{NO}_{3} \mathrm{~S}^{+}(\mathrm{M}+\mathrm{H})^{+}: 436.1189$, Found: 436.1190 .<smiles>[B]N(C/C=C\CC)Cc1ccco1</smiles> 
(Z)- $\mathbf{N}$-(Furan-2-ylmethyl)-4-methyl- $\boldsymbol{N}$-(pent-2-en-1-yl)benzenesulfonamide (1q). Colorless oil. ${ }^{1} \mathrm{H}$ NMR $\left(400 \mathrm{MHz}, \mathrm{CDCl}_{3}\right) \delta 7.66(\mathrm{~d}, J=8.3 \mathrm{~Hz}, 2 \mathrm{H}), 7.29-7.21(\mathrm{~m}, 3 \mathrm{H})$, $6.26(\mathrm{dd}, J=3.2,1.9 \mathrm{~Hz}, 1 \mathrm{H}), 6.14(\mathrm{~d}, J=3.2 \mathrm{~Hz}, 1 \mathrm{H}), 5.59-5.48(\mathrm{~m}, 1 \mathrm{H}), 5.23-5.12(\mathrm{~m}$, $1 \mathrm{H}), 4.38(\mathrm{~s}, 2 \mathrm{H}), 3.83(\mathrm{~d}, J=7.0 \mathrm{~Hz}, 2 \mathrm{H}), 2.40(\mathrm{~s}, 3 \mathrm{H}), 1.96(\mathrm{pd}, J=7.5,1.3 \mathrm{~Hz}, 2 \mathrm{H})$, $0.92(\mathrm{t}, J=7.5 \mathrm{~Hz}, 3 \mathrm{H}) .{ }^{13} \mathrm{C}$ NMR $\left(101 \mathrm{MHz}, \mathrm{CDCl}_{3}\right) \delta 150.0,143.1,142.4,137.3$, 136.6, 129.5, 127.3, 122.9, 110.4, 109.2, 43.7, 42.7, 21.5, 20.5, 14.1. HRMS (ESI) calcd. for $\mathrm{C}_{17} \mathrm{H}_{22} \mathrm{NO}_{3} \mathrm{~S}^{+}(\mathrm{M}+\mathrm{H})^{+}: 320.1315$, Found: 320.1313 .

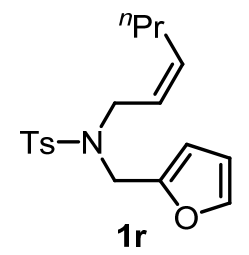

(Z)- $\mathbf{N}$-(Furan-2-ylmethyl)- $\boldsymbol{N}$-(hex-2-en-1-yl)-4-methylbenzenesulfonamide (1r). Colorless oil. ${ }^{1} \mathrm{H}$ NMR $\left(400 \mathrm{MHz}, \mathrm{CDCl}_{3}\right) \delta 7.66(\mathrm{~d}, J=8.3 \mathrm{~Hz}, 2 \mathrm{H}), 7.30-7.19(\mathrm{~m}, 3 \mathrm{H})$, $6.26(\mathrm{dd}, J=3.1,1.9 \mathrm{~Hz}, 1 \mathrm{H}), 6.14(\mathrm{~d}, J=3.1 \mathrm{~Hz}, 1 \mathrm{H}), 5.61-5.45(\mathrm{~m}, 1 \mathrm{H}), 5.27-5.16(\mathrm{~m}$, $1 \mathrm{H}), 4.38(\mathrm{~s}, 2 \mathrm{H}), 3.84(\mathrm{~d}, J=7.0 \mathrm{~Hz}, 2 \mathrm{H}), 2.40(\mathrm{~s}, 3 \mathrm{H}), 1.93(\mathrm{q}, J=7.0 \mathrm{~Hz}, 2 \mathrm{H})$, 1.39-1.23 (m, 2H), $0.85(\mathrm{t}, J=7.4 \mathrm{~Hz}, 3 \mathrm{H}) .{ }^{13} \mathrm{C} \mathrm{NMR}\left(101 \mathrm{MHz}, \mathrm{CDCl}_{3}\right) \delta 149.9,143.1$, 142.3, 137.3, 134.8, 129.5, 127.2, 123.7, 110.3, 109.2, 43.7, 42.6, 29.1, 22.6, 21.5, 13.7. HRMS (ESI) calcd. for $\mathrm{C}_{18} \mathrm{H}_{24} \mathrm{NO}_{3} \mathrm{~S}^{+}(\mathrm{M}+\mathrm{H})^{+}: 334.1471$, Found: 334.1473 .

\section{Optimization of the Reaction Conditions}

General procedure: In a glovebox, hydrosilane $(0.15 \mathrm{mmol}, 1.5$ equiv) and 1a $(0.1$ mmol, 1 equiv) were dissolved in dry toluene $(0.25 \mathrm{~mL})$ in an $8 \mathrm{~mL}$ vial. $\mathrm{B}\left(\mathrm{C}_{6} \mathrm{~F}_{5}\right)_{3}$ ( $0.005 \mathrm{mmol}, 5 \mathrm{~mol} \%$ ) was then added before the vial was capped and closed tightly. The reaction mixture was then stirred at $120{ }^{\circ} \mathrm{C}$ for $24 \mathrm{~h}$. After being allowed to cool to room temperature, one drop of $\mathrm{Et}_{3} \mathrm{~N}$ was added to the solution. After evaporation of the solvent in vacuo, the residue was analyzed with ${ }^{1} \mathrm{H}$ NMR spectroscopy to determine the yields of products using $\mathrm{CH}_{2} \mathrm{Br}_{2}(5 \mu \mathrm{L}, 0.071 \mathrm{mmol})$ as the internal standard.

\section{Substrate Scope}

General procedure: In a glovebox, ${ }^{i} \mathrm{Pr}_{3} \mathrm{SiH}(61 \mu \mathrm{L}, 0.3 \mathrm{mmol}, 1.5$ equiv $)$ or $\mathrm{Et}_{3} \mathrm{SiH}$ (48 $\mu \mathrm{L}, 0.3 \mathrm{mmol}, 1.5$ equiv) and a furan substrate $(0.2 \mathrm{mmol}, 1$ equiv) were dissolved in a dry solvent $\left(0.5 \mathrm{~mL}\right.$ of toluene or DCE) in an $8 \mathrm{~mL}$ vial. $\mathrm{B}\left(\mathrm{C}_{6} \mathrm{~F}_{5}\right)_{3}(5.1$ $\mathrm{mg}, 0.01 \mathrm{mmol}, 5 \mathrm{~mol} \%$ ) was then added before the vial was capped and closed tightly. The reaction mixture was stirred at the corresponding temperature for $24 \mathrm{~h}$. After being allowed to cool to room temperature, one drop of $\mathrm{Et}_{3} \mathrm{~N}$ was added to the solution. After evaporation of the solvent in vacuo, the residue was subjected to column chromatography on silica gel to give the corresponding product. The relative stereochemistry was determined by NOE experiment, X-ray crystallography or analogy to that of similar products. 


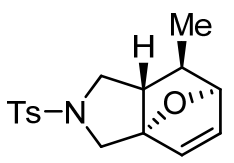

2a

( \pm )-7-Methyl-2-tosyl-1,2,3,6,7,7a-hexahydro-3a,6-epoxyisoindole (2a). Reaction conditions: no $\mathrm{B}\left(\mathrm{C}_{6} \mathrm{~F}_{5}\right)_{3}$, no hydrosilane, toluene, $120{ }^{\circ} \mathrm{C}$. White solid (4 mg, $7 \%$ yield). ${ }^{1} \mathrm{H}$ NMR (400 MHz, $\left.\mathrm{CDCl}_{3}\right) \delta 7.70(\mathrm{~d}, J=7.9 \mathrm{~Hz}, 2 \mathrm{H}), 7.31$ (d, J=7.9 Hz, 2H), 6.41 (d, $J=5.7 \mathrm{~Hz}, 1 \mathrm{H}), 6.35-6.28(\mathrm{~m}, 1 \mathrm{H}), 4.78-4.71(\mathrm{~m}, 1 \mathrm{H}), 3.97-3.84(\mathrm{~m}, 2 \mathrm{H}), 3.42(\mathrm{~d}, J=$ $12.1 \mathrm{~Hz}, 1 \mathrm{H}), 2.72(\mathrm{t}, J=9.3 \mathrm{~Hz}, 1 \mathrm{H}), 2.41(\mathrm{~s}, 3 \mathrm{H}), 2.10-2.01(\mathrm{~m}, 1 \mathrm{H}), 1.67-1.58(\mathrm{~m}$, $1 \mathrm{H}), 0.81(\mathrm{~d}, J=7.0 \mathrm{~Hz}, 3 \mathrm{H}) .{ }^{13} \mathrm{C} \mathrm{NMR}\left(101 \mathrm{MHz}, \mathrm{CDCl}_{3}\right) \delta 143.6,135.3,135.3$, 133.6, 129.8, 127.5, 96.0, 83.8, 53.0, 51.0, 49.7, 38.8, 21.6, 16.5. HRMS (ESI) calcd. for $\mathrm{C}_{16} \mathrm{H}_{20} \mathrm{NO}_{3} \mathrm{~S}^{+}(\mathrm{M}+\mathrm{H})^{+}:$306.1158, Found: 306.1158 .<smiles>[M]C1C2CN([3H])CC2=CC[C@@H]1OCC</smiles>

$3 a$

( \pm )-4-Methyl-2-tosyl-5-((triethylsilyl)oxy)-2,3,3a,4,5,6-hexahydro-1H-isoindole

(3a). Reaction conditions: $\mathrm{Et}_{3} \mathrm{SiH}$ (1.5 equiv), $\mathrm{B}\left(\mathrm{C}_{6} \mathrm{~F}_{5}\right)_{3}(5 \mathrm{~mol} \%)$, toluene, $120{ }^{\circ} \mathrm{C}$. White solid (38 mg, 45\% yield). ${ }^{1} \mathrm{H}$ NMR (400 MHz, $\left.\mathrm{CDCl}_{3}\right) \delta 7.69(\mathrm{~d}, J=8.0 \mathrm{~Hz}, 2 \mathrm{H})$, $7.32(\mathrm{~d}, J=8.0 \mathrm{~Hz}, 2 \mathrm{H}), 5.37(\mathrm{~s}, 1 \mathrm{H}), 3.99-3.91(\mathrm{~m}, 1 \mathrm{H}), 3.82-3.76(\mathrm{~m}, 1 \mathrm{H}), 3.67-3.59$ $(\mathrm{m}, 1 \mathrm{H}), 3.52-3.44(\mathrm{~m}, 1 \mathrm{H}), 2.59(\mathrm{t}, J=9.8 \mathrm{~Hz}, 1 \mathrm{H}), 2.42(\mathrm{~s}, 3 \mathrm{H}), 2.38-2.18(\mathrm{~m}, 2 \mathrm{H})$, 2.01-1.90 (m, 1H), 1.30-1.22 (m, 1H), 0.99-0.90 (m, 12H), 0.63-0.52 (m, 6H). ${ }^{13} \mathrm{C}$ NMR (101 MHz, $\left.\mathrm{CDCl}_{3}\right) \delta 143.6,136.1,133.8,129.9,127.7,118.1,72.6,53.5,51.1$, 46.3, 40.3, 35.9, 21.7, 16.3, 7.0, 5.1. HRMS (MALDI) calcd. for $\mathrm{C}_{22} \mathrm{H}_{35} \mathrm{NNaO}_{3} \mathrm{SSi}^{+}$ $(\mathrm{M}+\mathrm{Na})^{+}:$444.1999, Found: 444.2002. The relative stereochemistry of this compound was assigned by NOE experiment.

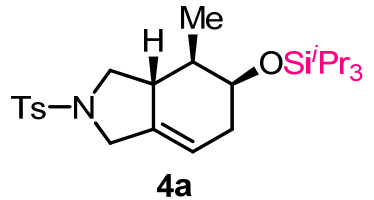

( \pm )-4-Methyl-2-tosyl-5-((triisopropylsilyl)oxy)-2,3,3a,4,5,6-hexahydro-1H-isoindol e (4a). Reaction conditions: ${ }^{i} \mathrm{Pr}_{3} \mathrm{SiH}$ (1.5 equiv), $\mathrm{B}\left(\mathrm{C}_{6} \mathrm{~F}_{5}\right)_{3}(5 \mathrm{~mol} \%)$, toluene, $110{ }^{\circ} \mathrm{C}$. White solid (79 mg, 85\% yield). ${ }^{1} \mathrm{H}$ NMR $\left(400 \mathrm{MHz}, \mathrm{CDCl}_{3}\right) \delta 7.70(\mathrm{~d}, J=8.0 \mathrm{~Hz}$, $2 \mathrm{H}), 7.30(\mathrm{~d}, J=8.0 \mathrm{~Hz}, 2 \mathrm{H}), 5.31(\mathrm{~s}, 1 \mathrm{H}), 4.09-3.95(\mathrm{~m}, 2 \mathrm{H}), 3.86-3.79(\mathrm{~m}, 1 \mathrm{H}), 3.67$ $(\mathrm{d}, J=13.5 \mathrm{~Hz}, 1 \mathrm{H}), 2.66-2.54(\mathrm{~m}, 1 \mathrm{H}), 2.55-2.38(\mathrm{~m}, 4 \mathrm{H}), 2.27-2.07(\mathrm{~m}, 2 \mathrm{H})$, $1.27-1.17(\mathrm{~m}, 1 \mathrm{H}), 1.12-0.84(\mathrm{~m}, 24 \mathrm{H}) .{ }^{13} \mathrm{C} \mathrm{NMR}\left(101 \mathrm{MHz}, \mathrm{CDCl}_{3}\right) \delta 143.4,136.6$, 133.9, 129.8, 127.6, 115.8, 70.2, 53.4, 51.4, 40.2, 38.5, 35.2, 21.6, 18.3, 18.2, 17.2, 12.9. HRMS (MALDI) calcd. for $\mathrm{C}_{25} \mathrm{H}_{41} \mathrm{NNaO}_{3} \mathrm{SSi}^{+}(\mathrm{M}+\mathrm{Na})^{+}: 486.2469$, Found: 486.2473. The structure and relative stereochemistry of this compound was determined by X-ray crystallography and NOE experiment.<smiles>[3H]N1CC2=CC[C@H](OCC)C([C@H](C)OC)C2C1</smiles> 
( \pm )-4-Propyl-2-tosyl-5-((triethylsilyl)oxy)-2,3,3a,4,5,6-hexahydro-1H-isoindole

(3b). Reaction conditions: $\mathrm{Et}_{3} \mathrm{SiH}$ (2.0 equiv), $\mathrm{B}\left(\mathrm{C}_{6} \mathrm{~F}_{5}\right)_{3}(5 \mathrm{~mol} \%), \mathrm{DCE}, 120{ }^{\circ} \mathrm{C}$. White solid (49 mg, 54\% yield). ${ }^{1} \mathrm{H} \mathrm{NMR}\left(400 \mathrm{MHz}, \mathrm{CDCl}_{3}\right) \delta 7.69$ (d, J = 8.1 Hz, 2H), $7.32(\mathrm{~d}, J=8.1 \mathrm{~Hz}, 2 \mathrm{H}), 5.32-5.38(\mathrm{~m}, 1 \mathrm{H}), 3.92(\mathrm{~d}, J=13.3 \mathrm{~Hz}, 1 \mathrm{H}), 3.85-3.79$ (m, 1H), 3.66- $3.55(\mathrm{~m}, 2 \mathrm{H}), 2.60(\mathrm{dd}, J=10.5,9.3 \mathrm{~Hz}, 1 \mathrm{H}), 2.43(\mathrm{~s}, 3 \mathrm{H}), 2.39-2.25(\mathrm{~m}$, 2H), 2.00-1.90 (m, 1H), 1.55-1.47 (m, 1H), 1.40-1.19 (m, 4H), 0.96-0.86 (m, 12H), 0.57 (q, $J=7.9 \mathrm{~Hz}, 6 \mathrm{H}) .{ }^{13} \mathrm{C}$ NMR $\left(101 \mathrm{MHz}, \mathrm{CDCl}_{3}\right) \delta 143.6,136.4,133.8,129.8$, $127.7,117.9,70.8,53.7,50.8,44.7,44.1,36.1,32.5,21.7,19.7,14.8,7.0,5.2$. HRMS (MALDI) calcd. for $\mathrm{C}_{24} \mathrm{H}_{39} \mathrm{NNaO}_{3} \mathrm{SSi}^{+}(\mathrm{M}+\mathrm{Na})^{+}$: 472.2312, Found: 472.2316. The structure and relative stereochemistry of this compound was determined by X-ray crystallography.

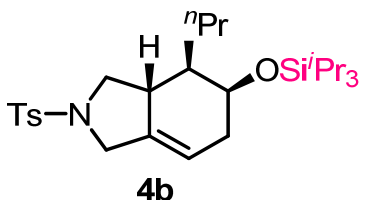

( \pm )-4-Propyl-2-tosyl-5-((triisopropylsilyl)oxy)-2,3,3a,4,5,6-hexahydro-1H-isoindol e (4b). Reaction conditions: ${ }^{i} \mathrm{Pr}_{3} \mathrm{SiH}$ (1.5 equiv), $\mathrm{B}\left(\mathrm{C}_{6} \mathrm{~F}_{5}\right)_{3}(5 \mathrm{~mol} \%)$, toluene, $110{ }^{\circ} \mathrm{C}$. White solid (91 mg, 93\% yield). ${ }^{1} \mathrm{H} \mathrm{NMR}\left(400 \mathrm{MHz}, \mathrm{CDCl}_{3}\right) \delta 7.71(\mathrm{~d}, J=8.0 \mathrm{~Hz}$, $2 \mathrm{H}), 7.30(\mathrm{~d}, J=8.0 \mathrm{~Hz}, 2 \mathrm{H}), 5.31(\mathrm{~s}, 1 \mathrm{H}), 4.18-4.13(\mathrm{~m}, 1 \mathrm{H}), 4.00(\mathrm{~d}, J=13.5 \mathrm{~Hz}$, $1 \mathrm{H}), 3.86-3.80(\mathrm{~m}, 1 \mathrm{H}), 3.67(\mathrm{~d}, J=13.5 \mathrm{~Hz}, 1 \mathrm{H}), 2.68-2.56(\mathrm{~m}, 1 \mathrm{H}), 2.55-2.45(\mathrm{~m}$, $1 \mathrm{H}), 2.41(\mathrm{~s}, 3 \mathrm{H}), 2.17-2.10(\mathrm{~m}, 2 \mathrm{H}), 1.56-1.44(\mathrm{~m}, 1 \mathrm{H}), 1.44-1.29(\mathrm{~m}, 1 \mathrm{H}), 1.20-1.10$ $(\mathrm{m}, 2 \mathrm{H}), 1.07-0.90(\mathrm{~m}, 22 \mathrm{H}), 0.86(\mathrm{t}, J=7.0 \mathrm{~Hz}, 3 \mathrm{H}) .{ }^{13} \mathrm{C} \mathrm{NMR}\left(101 \mathrm{MHz}, \mathrm{CDCl}_{3}\right) \delta$ 143.4, 136.9, 133.9, 129.8, 127.6, 115.8, 66.6, 53.6, 51.3, 43.5, 39.7, 35.2, 32.9, 21.6, 20.4, 18.3, 18.2, 14.4, 13.0. HRMS (MALDI) calcd. for $\mathrm{C}_{27} \mathrm{H}_{45} \mathrm{NNaO}_{3} \mathrm{SSi}^{+}(\mathrm{M}+\mathrm{Na})^{+}$: 514.2782, Found: 514.2783.<smiles>[3H]N1CC2=CC[C@H](OCC)C(C(C)C)C2C1</smiles>

( \pm )-4-Isopropyl-2-tosyl-5-((triethylsilyl)oxy)-2,3,3a,4,5,6-hexahydro-1H-isoindole (3c). Reaction conditions: $\mathrm{Et}_{3} \mathrm{SiH}$ (1.5 equiv), $\mathrm{B}\left(\mathrm{C}_{6} \mathrm{~F}_{5}\right)_{3}(5 \mathrm{~mol} \%)$, toluene, $120{ }^{\circ} \mathrm{C}$. White solid (48 mg, 53\% yield). ${ }^{1} \mathrm{H}$ NMR (400 MHz, $\left.\mathrm{CDCl}_{3}\right) \delta 7.70(\mathrm{~d}, J=8.2 \mathrm{~Hz}$, $2 \mathrm{H}), 7.32(\mathrm{~d}, J=8.2 \mathrm{~Hz}, 2 \mathrm{H}), 5.38-5.33(\mathrm{~m}, 1 \mathrm{H}), 3.93-3.81(\mathrm{~m}, 2 \mathrm{H}), 3.67(\mathrm{td}, J=9.7$, $5.8 \mathrm{~Hz}, 1 \mathrm{H}), 3.58(\mathrm{~d}, J=13.4 \mathrm{~Hz}, 1 \mathrm{H}), 2.67-2.56(\mathrm{~m}, 1 \mathrm{H}), 2.43(\mathrm{~s}, 3 \mathrm{H}), 2.40-2.20(\mathrm{~m}$, $3 \mathrm{H}), 2.02-1.91(\mathrm{~m}, 1 \mathrm{H}), 1.27-1.17(\mathrm{~m}, 1 \mathrm{H}), 0.95-0.89(\mathrm{~m}, 12 \mathrm{H}), 0.81(\mathrm{~d}, J=7.0 \mathrm{~Hz}, 3 \mathrm{H})$, 0.57 (q, $J=7.8 \mathrm{~Hz}, 6 \mathrm{H}) .{ }^{13} \mathrm{C}$ NMR $\left(101 \mathrm{MHz}, \mathrm{CDCl}_{3}\right) \delta 143.6,136.8,133.9,129.9$, $127.7,117.5,69.3,54.0,50.3,50.2,40.2,36.2,25.3,21.9,21.7,17.0,7.0,5.3$. HRMS (MALDI) calcd. for $\mathrm{C}_{24} \mathrm{H}_{39} \mathrm{NNaO}_{3} \mathrm{SSi}^{+}(\mathrm{M}+\mathrm{Na})^{+}: 472.2312$, Found: 472.2316 .

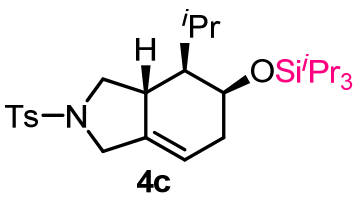

( \pm )-4-Isopropyl-2-tosyl-5-((triisopropylsilyl)oxy)-2,3,3a,4,5,6-hexahydro-1H-isoin dole (4c). Reaction conditions: ${ }^{i} \mathrm{Pr}_{3} \mathrm{SiH}$ (1.5 equiv), $\mathrm{B}\left(\mathrm{C}_{6} \mathrm{~F}_{5}\right)_{3}(5 \mathrm{~mol} \%)$, toluene, 
$120{ }^{\circ} \mathrm{C}$. Colorless oil. (42 mg, 43\% yield). ${ }^{1} \mathrm{H}$ NMR (400 MHz, $\left.\mathrm{CDCl}_{3}\right) \delta 7.71(\mathrm{~d}, J=$ $7.7 \mathrm{~Hz}, 2 \mathrm{H}), 7.31(\mathrm{~d}, J=7.7 \mathrm{~Hz}, 2 \mathrm{H}), 5.33(\mathrm{~s}, 1 \mathrm{H}), 4.31-4.18(\mathrm{~m}, 1 \mathrm{H}), 4.02-3.88(\mathrm{~m}$, $2 \mathrm{H}), 3.67(\mathrm{~d}, J=13.5 \mathrm{~Hz}, 1 \mathrm{H}), 2.73-2.62(\mathrm{~m}, 1 \mathrm{H}), 2.58-2.49(\mathrm{~m}, 1 \mathrm{H}), 2.41(\mathrm{~s}, 3 \mathrm{H})$, 2.18-2.09 (m, 2H), 1.82-1.68 (m, 1H), 1.36-1.25 (m, 1H), 1.00-0.88 (m, 27H). ${ }^{13} \mathrm{C}$ NMR $\left(101 \mathrm{MHz}, \mathrm{CDCl}_{3}\right) \delta 143.5,137.4,134.0,129.8,127.6,115.9,67.9,54.4,50.8$, 49.7, 38.2, 35.7, 29.8, 21.7, 21.6, 21.5, 18.4, 18.2, 13.3. HRMS (MALDI) calcd. for $\mathrm{C}_{27} \mathrm{H}_{45} \mathrm{NNaO}_{3} \mathrm{SSi}^{+}(\mathrm{M}+\mathrm{Na})^{+}:$514.2782, Found: 514.2785.<smiles>CCO[C@H]1CC=C2CN([As])CC2C1Br</smiles>

(士)-4-Benzyl-2-tosyl-5-((triethylsilyl)oxy)-2,3,3a,4,5,6-hexahydro-1H-isoindole

(3d). Reaction conditions: $\mathrm{Et}_{3} \mathrm{SiH}$ (1.5 equiv), $\mathrm{B}\left(\mathrm{C}_{6} \mathrm{~F}_{5}\right)_{3}(5 \mathrm{~mol} \%)$, toluene, $120{ }^{\circ} \mathrm{C}$. White solid (41 mg, 41\% yield). ${ }^{1} \mathrm{H}$ NMR (400 MHz, $\left.\mathrm{CDCl}_{3}\right) \delta 7.50$ (d, $J=7.9 \mathrm{~Hz}$, $2 \mathrm{H}), 7.34-7.22(\mathrm{~m}, 5 \mathrm{H}), 7.15(\mathrm{~d}, J=7.0 \mathrm{~Hz}, 2 \mathrm{H}), 5.38(\mathrm{~s}, 1 \mathrm{H}), 3.78(\mathrm{~d}, J=13.2 \mathrm{~Hz}, 1 \mathrm{H})$, 3.67-3.59 (m, 1H), $3.53(\mathrm{~d}, J=13.4 \mathrm{~Hz}, 1 \mathrm{H}), 3.31(\mathrm{dd}, J=13.6,2.5 \mathrm{~Hz}, 1 \mathrm{H}), 2.95-2.88$ $(\mathrm{m}, 1 \mathrm{H}), 2.43(\mathrm{~s}, 3 \mathrm{H}), 2.41-2.32(\mathrm{~m}, 1 \mathrm{H}), 2.25(\mathrm{dd}, J=13.4,9.7 \mathrm{~Hz}, 1 \mathrm{H}), 2.16-2.01(\mathrm{~m}$, $3 \mathrm{H}), 1.66-1.57(\mathrm{~m}, 1 \mathrm{H}), 0.97(\mathrm{t}, J=7.9 \mathrm{~Hz}, 9 \mathrm{H}), 0.62(\mathrm{q}, J=7.9 \mathrm{~Hz}, 6 \mathrm{H}) .{ }^{13} \mathrm{C} \mathrm{NMR}$ $\left(101 \mathrm{MHz}, \mathrm{CDCl}_{3}\right) \delta 143.4,140.6,136.4,133.9,129.7,129.4,128.5,127.7,126.5$, 117.9, 71.3, 53.4, 50.3, 47.3, 44.6, 37.6, 35.6, 21.7, 7.1, 5.4. HRMS (MALDI) calcd. for $\mathrm{C}_{28} \mathrm{H}_{39} \mathrm{NNaO}_{3} \mathrm{SSi}^{+}(\mathrm{M}+\mathrm{Na})^{+}:$520.2312, Found: 520.2315 .<smiles>[B]N1CC2=CCC(O[GaH2])C(Br)C2C1</smiles>

( \pm )-4-Benzyl-2-tosyl-5-((triisopropylsilyl)oxy)-2,3,3a,4,5,6-hexahydro-1H-isoindol e (4d). Reaction conditions: ${ }^{i} \mathrm{Pr}_{3} \mathrm{SiH}$ (1.5 equiv), $\mathrm{B}\left(\mathrm{C}_{6} \mathrm{~F}_{5}\right)_{3}(5 \mathrm{~mol} \%)$, toluene, $110{ }^{\circ} \mathrm{C}$. White solid (90 mg, 84\% yield). ${ }^{1} \mathrm{H}$ NMR (400 MHz, $\left.\mathrm{CDCl}_{3}\right) \delta 7.64$ (d, J=8.0 Hz, 2H), 7.34-7.22 (m, 5H), $7.12(\mathrm{~d}, J=7.2 \mathrm{~Hz}, 2 \mathrm{H}), 5.34(\mathrm{~s}, 1 \mathrm{H}), 4.04-3.92(\mathrm{~m}, 2 \mathrm{H}), 3.66$ $(\mathrm{d}, J=13.7 \mathrm{~Hz}, 1 \mathrm{H}), 3.51-3.44(\mathrm{~m}, 1 \mathrm{H}), 2.79(\mathrm{dd}, J=13.8,8.4 \mathrm{~Hz}, 1 \mathrm{H}), 2.63(\mathrm{dd}, J=$ $13.8,6.4 \mathrm{~Hz}, 1 \mathrm{H}), 2.56-2.48(\mathrm{~m}, 1 \mathrm{H}), 2.44-2.38(\mathrm{~m}, 4 \mathrm{H}), 2.18-2.08(\mathrm{~m}, 2 \mathrm{H}), 1.51-1.43$ (m, 1H), 1.10-0.94 (m, 21H). $\left.{ }^{13} \mathrm{C} \mathrm{NMR} \mathrm{(101} \mathrm{MHz,} \mathrm{CDCl}_{3}\right) \delta 143.4,140.2,136.9,133.8$, 129.7, 129.1, 128.5, 127.6, 126.4, 116.0, 67.4, 53.4, 51.1, 45.7, 39.5, 37.7, 35.1, 21.7, 18.4, 18.3, 13.1. HRMS (MALDI) calcd. for $\mathrm{C}_{31} \mathrm{H}_{45} \mathrm{NNaO}_{3} \mathrm{SSi}^{+}(\mathrm{M}+\mathrm{Na})^{+}: 562.2782$, Found: 562.2785.<smiles></smiles>

( \pm )-4-(2-Bromoethyl)-2-tosyl-5-((triisopropylsilyl)oxy)-2,3,3a,4,5,6-hexahydro-1H -isoindole (4e). Reaction conditions: ${ }^{i} \mathrm{Pr}_{3} \mathrm{SiH}$ (1.5 equiv), $\mathrm{B}\left(\mathrm{C}_{6} \mathrm{~F}_{5}\right)_{3}$ (5 mol \%), toluene, $110{ }^{\circ} \mathrm{C}$. Colorless oil (69 mg, $62 \%$ yield). ${ }^{1} \mathrm{H}$ NMR $\left(400 \mathrm{MHz}, \mathrm{CDCl}_{3}\right) \delta 7.71$ 
(d, $J=8.1 \mathrm{~Hz}, 2 \mathrm{H}), 7.32(\mathrm{~d}, J=8.1 \mathrm{~Hz}, 2 \mathrm{H}), 5.38-5.33(\mathrm{~m}, 1 \mathrm{H}), 4.17-4.13(\mathrm{~m}, 1 \mathrm{H}), 4.03$ $(\mathrm{d}, J=13.5 \mathrm{~Hz}, 1 \mathrm{H}), 3.86-3.79(\mathrm{~m}, 1 \mathrm{H}), 3.67(\mathrm{~d}, J=13.5 \mathrm{~Hz}, 1 \mathrm{H}), 3.50-3.42(\mathrm{~m}, 1 \mathrm{H})$, 3.42-3.34 (m, 1H), $2.66(\mathrm{dd}, J=11.1,8.9 \mathrm{~Hz}, 1 \mathrm{H}), 2.60-2.50(\mathrm{~m}, 1 \mathrm{H}), 2.42(\mathrm{~s}, 3 \mathrm{H})$, 2.24-2.17 (m, 2H), 2.16-2.07 (m, 1H), 1.84-1.73 (m, 1H), $1.38(\mathrm{td}, J=9.5,3.7 \mathrm{~Hz}, 1 \mathrm{H})$, 1.03-0.94 (m, 21H). $\left.{ }^{13} \mathrm{C} \mathrm{NMR} \mathrm{(101} \mathrm{MHz,} \mathrm{CDCl}_{3}\right) \delta 143.6,136.5,133.7,129.8,127.6$, 115.9, 66.3, 53.1, 51.2, 41.9, 39.1, 34.9, 33.7, 31.5, 21.7, 18.3, 18.2, 13.0. HRMS (MALDI) calcd. for $\mathrm{C}_{26} \mathrm{H}_{43} \mathrm{BrNO}_{3} \mathrm{SSi}^{+}(\mathrm{M}+\mathrm{H})^{+}$: 556.1911, Found: 556.1915.

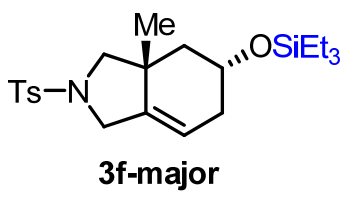

( \pm )-3a-Methyl-2-tosyl-5-((triethylsilyl)oxy)-2,3,3a,4,5,6-hexahydro-1H-isoindole

(3f). Reaction conditions: $\mathrm{Et}_{3} \mathrm{SiH}$ (1.5 equiv), $\mathrm{B}\left(\mathrm{C}_{6} \mathrm{~F}_{5}\right)_{3}(5 \mathrm{~mol} \%), \mathrm{DCE}, 110{ }^{\circ} \mathrm{C}$. Colorless oil (77 mg, 91\% yield), $\mathrm{dr}=8.2: 1.0$. Major: ${ }^{1} \mathrm{H}$ NMR $\left(400 \mathrm{MHz}, \mathrm{CDCl}_{3}\right) \delta$ $7.69(\mathrm{~d}, J=8.2 \mathrm{~Hz}, 2 \mathrm{H}), 7.30(\mathrm{~d}, J=8.2 \mathrm{~Hz}, 2 \mathrm{H}), 5.32-5.28(\mathrm{~m}, 1 \mathrm{H}), 4.03-3.93(\mathrm{~m}, 2 \mathrm{H})$, $3.64(\mathrm{dd}, J=12.9,1.1 \mathrm{~Hz}, 1 \mathrm{H}), 3.39(\mathrm{~d}, J=8.9 \mathrm{~Hz}, 1 \mathrm{H}), 2.70$ (d, $J=8.9 \mathrm{~Hz}, 1 \mathrm{H}), 2.41$ (s, 3H), 2.35-2.25 (m, 1H), 1.94-1.84 (m, 1H), $1.78(\mathrm{dd}, J=11.9,3.6 \mathrm{~Hz}, 1 \mathrm{H}), 1.27(\mathrm{t}, J$ $=11.9 \mathrm{~Hz}, 1 \mathrm{H}), 0.99(\mathrm{~s}, 3 \mathrm{H}), 0.92(\mathrm{t}, J=7.9 \mathrm{~Hz}, 9 \mathrm{H}), 0.56(\mathrm{q}, J=7.9 \mathrm{~Hz}, 6 \mathrm{H}) .{ }^{13} \mathrm{C} \mathrm{NMR}$ $\left(101 \mathrm{MHz}, \mathrm{CDCl}_{3}\right) \delta 143.5,139.2,134.1,129.7,127.5,118.0,65.4,61.5,49.9,42.7$, 42.5, 35.4, 24.5, 21.6, 6.9, 4.9. Minor (distinguishable peaks): ${ }^{1} \mathrm{H}$ NMR (400 MHz, $\left.\mathrm{CDCl}_{3}\right) \delta 5.35(\mathrm{~s}, 1 \mathrm{H}), 3.32(\mathrm{~d}, J=8.8 \mathrm{~Hz}, 1 \mathrm{H}), 1.46-1.38(\mathrm{~m}, 1 \mathrm{H}), 1.03(\mathrm{~s}, 3 \mathrm{H})$. HRMS (MALDI) calcd. for $\mathrm{C}_{22} \mathrm{H}_{35} \mathrm{NNaO}_{3} \mathrm{SSi}^{+}(\mathrm{M}+\mathrm{Na})^{+}$: 444.1999, Found: 444.2002. The relative stereochemistry of this major compound was assigned by NOE experiment.

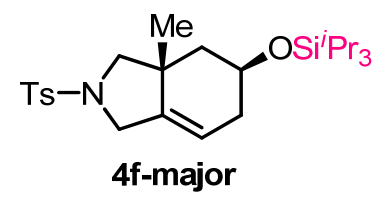

( \pm )-3a-Methyl-2-tosyl-5-((triisopropylsilyl)oxy)-2,3,3a,4,5,6-hexahydro-1H-isoind ole (4f). Reaction conditions: ${ }^{i} \mathrm{Pr}_{3} \mathrm{SiH}$ (1.5 equiv), $\mathrm{B}\left(\mathrm{C}_{6} \mathrm{~F}_{5}\right)_{3}(5 \mathrm{~mol} \%)$, toluene, $110{ }^{\circ} \mathrm{C}$. Colorless oil ( $85 \mathrm{mg}, 92 \%$ yield), $\mathrm{dr}=3.3: 1.0$. Major: ${ }^{1} \mathrm{H}$ NMR $\left(400 \mathrm{MHz}, \mathrm{CDCl}_{3}\right) \delta$ $7.70(\mathrm{~d}, J=8.1 \mathrm{~Hz}, 2 \mathrm{H}), 7.30(\mathrm{~d}, J=8.1 \mathrm{~Hz}, 2 \mathrm{H}), 5.38-5.32(\mathrm{~m}, 1 \mathrm{H}), 4.13-4.05(\mathrm{~m}, 1 \mathrm{H})$, 4.02-3.95 (m, 1H), $3.72(\mathrm{~d}, J=12.5 \mathrm{~Hz}, 1 \mathrm{H}), 3.33(\mathrm{~d}, J=8.9 \mathrm{~Hz}, 1 \mathrm{H}), 2.76(\mathrm{~d}, J=8.9$ $\mathrm{Hz}, 1 \mathrm{H}), 2.41(\mathrm{~s}, 3 \mathrm{H}), 2.30-2.20(\mathrm{~m}, 1 \mathrm{H}), 2.12-2.02(\mathrm{~m}, 1 \mathrm{H}), 1.81(\mathrm{dd}, J=13.6,5.2 \mathrm{~Hz}$, $1 \mathrm{H}), 1.45(\mathrm{dd}, J=13.6,3.5 \mathrm{~Hz}, 1 \mathrm{H}), 1.07-0.96(\mathrm{~m}, 24 \mathrm{H}) .{ }^{13} \mathrm{C} \mathrm{NMR}\left(101 \mathrm{MHz}, \mathrm{CDCl}_{3}\right)$ $\delta 143.4,139.4,134.5,129.7,127.5,117.0,65.9,62.0,50.5,40.0,39.9,34.3,25.3,21.6$, 18.2, 18.1, 12.3. HRMS (MALDI) calcd. for $\mathrm{C}_{25} \mathrm{H}_{41} \mathrm{NNaO}_{3} \mathrm{SSi}^{+}(\mathrm{M}+\mathrm{Na})^{+}$: 486.2469, Found: 486.2470. The relative stereochemistry of this compound was assigned by NOE experiment.

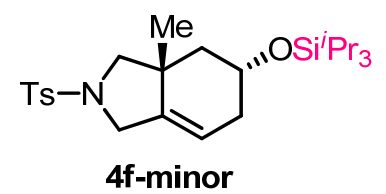

Minor: ${ }^{1} \mathrm{H}$ NMR $\left(400 \mathrm{MHz}, \mathrm{CDCl}_{3}\right) \delta 7.70(\mathrm{~d}, J=8.1 \mathrm{~Hz}, 2 \mathrm{H}), 7.31(\mathrm{~d}, J=8.1 \mathrm{~Hz}, 2 \mathrm{H})$, 5.34-5.30 (m, 1H), 4.16-4.06 (m, 1H), 3.99 (dd, $J=13.0,2.6 \mathrm{~Hz} 1 \mathrm{H}), 3.65$ (d, $J=13.0$ 
$\mathrm{Hz}, 1 \mathrm{H}), 3.41(\mathrm{~d}, J=8.9 \mathrm{~Hz}, 1 \mathrm{H}), 2.72(\mathrm{~d}, J=8.9 \mathrm{~Hz}, 1 \mathrm{H}), 2.45-2.34(\mathrm{~m}, 4 \mathrm{H}), 1.97-1.85$ $(\mathrm{m}, 2 \mathrm{H}), 1.32-1.25(\mathrm{~m}, 1 \mathrm{H}), 1.06-1.02(\mathrm{~m}, 21 \mathrm{H}), 1.00(\mathrm{~s}, 3 \mathrm{H}) .{ }^{13} \mathrm{C}$ NMR $(101 \mathrm{MHz}$, $\left.\mathrm{CDCl}_{3}\right) \delta 143.5,139.4,134.6,129.8,127.6,118.1,65.7,61.6,50.1,42.8,42.7,35.8$, 24.6, 21.6, 18.2, 12.5. HRMS (MALDI) calcd. for $\mathrm{C}_{25} \mathrm{H}_{41} \mathrm{NNaO}_{3} \mathrm{SSi}^{+}(\mathrm{M}+\mathrm{Na})^{+}$: 486.2469, Found: 486.2472.

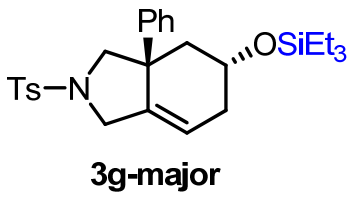

\section{( \pm )-3a-Phenyl-2-tosyl-5-((triethylsilyl)oxy)-2,3,3a,4,5,6-hexahydro-1H-isoindole}

(3g). Reaction conditions: $\mathrm{Et}_{3} \mathrm{SiH}$ (1.5 equiv), $\mathrm{B}\left(\mathrm{C}_{6} \mathrm{~F}_{5}\right)_{3}(5 \mathrm{~mol} \%)$, toluene, $120{ }^{\circ} \mathrm{C}$. White solid (62 mg, 64\% yield), $\mathrm{dr}=6.8: 1.0$. Major: ${ }^{1} \mathrm{H}$ NMR $\left(400 \mathrm{MHz}, \mathrm{CDCl}_{3}\right) \delta$ $7.39(\mathrm{~d}, J=8.1 \mathrm{~Hz}, 2 \mathrm{H}), 7.19-7.12(\mathrm{~m}, 3 \mathrm{H}), 7.08-7.01(\mathrm{~m}, 4 \mathrm{H}), 5.76(\mathrm{~s}, 1 \mathrm{H}), 4.04(\mathrm{~d}, J$ $=9.7 \mathrm{~Hz}, 1 \mathrm{H}), 3.93(\mathrm{dd}, J=12.9,2.4 \mathrm{~Hz} 1 \mathrm{H}), 3.83(\mathrm{~d}, J=12.9 \mathrm{~Hz}, 1 \mathrm{H}), 3.49-3.39(\mathrm{~m}$, $1 \mathrm{H}), 3.19(\mathrm{~d}, J=9.7 \mathrm{~Hz}, 1 \mathrm{H}), 2.37-2.28(\mathrm{~m}, 4 \mathrm{H}), 2.09(\mathrm{dd}, J=11.7,3.0 \mathrm{~Hz}, 1 \mathrm{H})$, 2.06-1.95 (m, 1H), $1.58(\mathrm{t}, J=11.7 \mathrm{~Hz}, 1 \mathrm{H}), 0.71(\mathrm{t}, J=7.9 \mathrm{~Hz}, 9 \mathrm{H}), 0.30(\mathrm{q}, J=7.9 \mathrm{~Hz}$, $6 \mathrm{H}) .{ }^{13} \mathrm{C}$ NMR $\left(101 \mathrm{MHz}, \mathrm{CDCl}_{3}\right) \delta 143.9,143.0,136.9,133.9,129.4,128.4,127.1$, $126.8,126.6,122.4,64.7,62.2,52.0,50.8,44.4,35.3,21.5,6.6,4.6$. HRMS (MALDI) calcd. for $\mathrm{C}_{27} \mathrm{H}_{37} \mathrm{NNaO}_{3} \mathrm{SSi}^{+}(\mathrm{M}+\mathrm{Na})^{+}$: 506.2156, Found: 506.2158. The relative stereochemistry of this compound was assigned by NOE experiment.<smiles>CCOC1CC2CC3(CCCC3)CN([As])CC2(c2ccccc2)C1</smiles>

Minor: ${ }^{1} \mathrm{H}$ NMR (400 MHz, $\left.\mathrm{CDCl}_{3}\right) \delta 7.35(\mathrm{~d}, J=8.2 \mathrm{~Hz}, 2 \mathrm{H}), 7.10-7.01(\mathrm{~m}, 7 \mathrm{H})$, 5.82-5.77 (m, 1H), $4.02(\mathrm{~d}, J=9.5 \mathrm{~Hz}, 1 \mathrm{H}), 3.97-3.85(\mathrm{~m}, 3 \mathrm{H}), 3.19(\mathrm{~d}, J=9.5 \mathrm{~Hz}, 1 \mathrm{H})$, 2.37-2.24 (m, 4H), 2.15-2.01 (m, 2H), $1.78(\mathrm{dd}, J=13.5,3.1 \mathrm{~Hz}, 1 \mathrm{H}), 0.73(\mathrm{t}, J=7.9$ $\mathrm{Hz}, 9 \mathrm{H}), 0.26(\mathrm{q}, J=7.9 \mathrm{~Hz}, 6 \mathrm{H}) .{ }^{13} \mathrm{C} \mathrm{NMR}\left(101 \mathrm{MHz}, \mathrm{CDCl}_{3}\right) \delta 145.4,142.9,137.1$, 134.5, 129.4, 128.1, 127.1, 126.6, 125.8, 121.6, 65.5, 62.7, 51.4, 48.6, 42.0, 34.5, 21.5, 6.8, 4.5. HRMS (ESI) calcd. for $\mathrm{C}_{27} \mathrm{H}_{37} \mathrm{NNaO}_{3} \mathrm{SSi}^{+}(\mathrm{M}+\mathrm{Na})^{+}: 506.2156$, Found: 506.2160 .

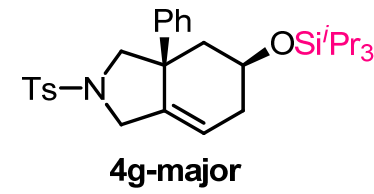

( \pm )-3a-Phenyl-2-tosyl-5-((triisopropylsilyl)oxy)-2,3,3a,4,5,6-hexahydro-1H-isoind ole (4g). Reaction conditions: ${ }^{i} \mathrm{Pr}_{3} \mathrm{SiH}$ (1.5 equiv), $\mathrm{B}\left(\mathrm{C}_{6} \mathrm{~F}_{5}\right)_{3}$ (5 mol \%), toluene, $110{ }^{\circ} \mathrm{C}$. White solid (95 mg, 90\% yield), $\mathrm{dr}=8.4: 1.0$. Major: ${ }^{1} \mathrm{H}$ NMR (400 MHz, $\left.\mathrm{CDCl}_{3}\right) \delta 7.37(\mathrm{~d}, J=7.9 \mathrm{~Hz}, 2 \mathrm{H}), 7.10-7.02(\mathrm{~m}, 7 \mathrm{H}), 5.84-5.78(\mathrm{~m}, 1 \mathrm{H}), 4.07-3.97(\mathrm{~m}$, $2 \mathrm{H}), 3.90-3.85(\mathrm{~m}, 2 \mathrm{H}), 3.19(\mathrm{~d}, J=9.5 \mathrm{~Hz}, 1 \mathrm{H}), 2.39-2.29(\mathrm{~m}, 4 \mathrm{H}), 2.15-2.03(\mathrm{~m}, 2 \mathrm{H})$, $1.89(\mathrm{dd}, J=13.7,3.5 \mathrm{~Hz}, 1 \mathrm{H}), 0.91-0.78(\mathrm{~m}, 21 \mathrm{H}) .{ }^{13} \mathrm{C} \mathrm{NMR}\left(101 \mathrm{MHz}, \mathrm{CDCl}_{3}\right) \delta$ $145.1,142.9,137.3,134.5,129.4,128.4,127.1,126.4,126.0,121.6,66.2,62.0,51.3$, 49.0, 42.4, 34.5, 21.5, 18.1, 18.0, 12.1. Minor (distinguishable peaks): ${ }^{1} \mathrm{H}$ NMR (400 $\left.\mathrm{MHz}_{\mathrm{CDCl}}\right) \delta 7.40(\mathrm{~d}, J=8.2 \mathrm{~Hz}, 2 \mathrm{H}), 5.78-5.75(\mathrm{~m}, 1 \mathrm{H}), 4.07(\mathrm{~d}, J=10.1 \mathrm{~Hz}, 1 \mathrm{H})$, 
$1.82(\mathrm{dd}, J=13.6,3.1 \mathrm{~Hz}, 1 \mathrm{H}), 1.62-1.54(\mathrm{~m}, 1 \mathrm{H})$. HRMS (MALDI) calcd. for $\mathrm{C}_{30} \mathrm{H}_{43} \mathrm{NNaO}_{3} \mathrm{SSi}^{+} \quad(\mathrm{M}+\mathrm{Na})^{+}:$548.2625, Found: 548.2630. The relative stereochemistry of this major compound was assigned by NOE experiment.

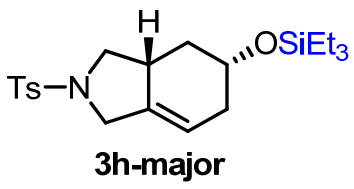

( \pm )-2-Tosyl-5-((triethylsilyl)oxy)-2,3,3a,4,5,6-hexahydro-1H-isoindole

(3h). Reaction conditions: $\mathrm{Et}_{3} \mathrm{SiH}$ (1.5 equiv), $\mathrm{B}\left(\mathrm{C}_{6} \mathrm{~F}_{5}\right)_{3}(5 \mathrm{~mol} \%), \mathrm{DCE}, 80^{\circ} \mathrm{C}$. White solid (50 mg, 61\% yield) $, \mathrm{dr}=12.0: 1.0$. Major: ${ }^{1} \mathrm{H}$ NMR $\left(400 \mathrm{MHz}, \mathrm{CDCl}_{3}\right) \delta 7.69(\mathrm{~d}, J=$ $8.2 \mathrm{~Hz}, 2 \mathrm{H}), 7.31(\mathrm{~d}, J=8.2 \mathrm{~Hz}, 2 \mathrm{H}), 5.43-5.38(\mathrm{~m}, 1 \mathrm{H}), 3.96-3.89(\mathrm{~m}, 1 \mathrm{H}), 3.88-3.79$ $(\mathrm{m}, 1 \mathrm{H}), 3.78-3.68(\mathrm{~m}, 1 \mathrm{H}), 3.62(\mathrm{~d}, J=13.4 \mathrm{~Hz}, 1 \mathrm{H}), 2.62-2.52(\mathrm{~m}, 2 \mathrm{H}), 2.42(\mathrm{~s}, 3 \mathrm{H})$, 2.33-2.23 (m, 1H), 2.01-1.87 (m, 2H), 1.18-1.08 (m, 1H), $0.92(\mathrm{t}, J=7.9 \mathrm{~Hz}, 9 \mathrm{H}), 0.56$ $(\mathrm{q}, J=7.9 \mathrm{~Hz}, 6 \mathrm{H}) .{ }^{13} \mathrm{C} \mathrm{NMR}\left(101 \mathrm{MHz}, \mathrm{CDCl}_{3}\right) \delta 143.6,136.3,133.8,129.8,127.7$, $118.4,67.7,53.9,50.6,39.4,35.8,35.2,21.7,6.9,4.9$. Minor (distinguishable peaks): ${ }^{1} \mathrm{H}$ NMR (400 MHz, $\left.\mathrm{CDCl}_{3}\right) \delta 5.37(\mathrm{~s}, 1 \mathrm{H}), 4.01(\mathrm{~d}, J=13.5 \mathrm{~Hz}, 1 \mathrm{H}), 2.23-2.14(\mathrm{~m}$, 1H). HRMS (MALDI) calcd. for $\mathrm{C}_{21} \mathrm{H}_{33} \mathrm{NNaO}_{3} \mathrm{SSi}^{+}(\mathrm{M}+\mathrm{Na})^{+}: 430.1843$, Found: 430.1846. The relative stereochemistry of this compound was assigned by NOE experiment.

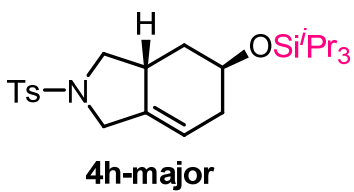

( \pm )-2-Tosyl-5-((triisopropylsilyl)oxy)-2,3,3a,4,5,6-hexahydro-1H-isoindole (4h). Reaction conditions: ${ }^{i} \mathrm{Pr}_{3} \mathrm{SiH}$ (1.5 equiv), $\mathrm{B}\left(\mathrm{C}_{6} \mathrm{~F}_{5}\right)_{3}(5 \mathrm{~mol} \%)$, toluene, $80{ }^{\circ} \mathrm{C}$. White solid (79 mg, 88\% yield), dr = 2.4:1.0. Major: ${ }^{1} \mathrm{H}$ NMR $\left(400 \mathrm{MHz}, \mathrm{CDCl}_{3}\right) \delta 7.71(\mathrm{~d}, J$ $=8.0 \mathrm{~Hz}, 2 \mathrm{H}), 7.30(\mathrm{~d}, J=8.0 \mathrm{~Hz}, 2 \mathrm{H}), 5.36(\mathrm{~s}, 1 \mathrm{H}), 4.23(\mathrm{~s}, 1 \mathrm{H}), 4.03(\mathrm{~d}, J=13.5 \mathrm{~Hz}$, $1 \mathrm{H}), 3.78(\mathrm{t}, J=8.3 \mathrm{~Hz}, 1 \mathrm{H}), 3.68(\mathrm{~d}, J=13.5 \mathrm{~Hz}, 1 \mathrm{H}), 2.78-2.66(\mathrm{~m}, 1 \mathrm{H}), 2.65-2.56(\mathrm{~m}$, $1 \mathrm{H}), 2.41(\mathrm{~s}, 3 \mathrm{H}), 2.18(\mathrm{~d}, J=18.5 \mathrm{~Hz}, 1 \mathrm{H}), 2.06(\mathrm{~d}, J=18.5 \mathrm{~Hz}, 1 \mathrm{H}), 2.01-1.93(\mathrm{~m}$, 1H), 1.05-0.94 (m, 22H). ${ }^{13} \mathrm{C}$ NMR $\left(101 \mathrm{MHz}, \mathrm{CDCl}_{3}\right) \delta 143.5,136.6,133.9,129.8$, 127.7, 116.3, 64.7, 54.1, 51.2, 34.2, 33.4, 33.2, 21.7, 18.2, 12.3. HRMS (MALDI) calcd. for $\mathrm{C}_{24} \mathrm{H}_{39} \mathrm{NNaO}_{3} \mathrm{SSi}^{+}(\mathrm{M}+\mathrm{Na})^{+}$: 472.2312, Found: 472.2315 .

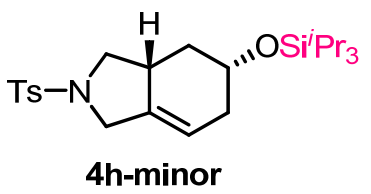

Minor: ${ }^{1} \mathrm{H}$ NMR $\left(400 \mathrm{MHz}, \mathrm{CDCl}_{3}\right) \delta 7.69(\mathrm{~d}, J=8.1 \mathrm{~Hz}, 2 \mathrm{H}), 7.32(\mathrm{~d}, J=8.1 \mathrm{~Hz}, 2 \mathrm{H})$, 5.44-5.39 (m, 1H), 3.99-3.89 (m, 2H), 3.79-3.69 (m, 1H), $3.62(\mathrm{~d}, J=13.4 \mathrm{~Hz}, 1 \mathrm{H})$, 2.63-2.51 (m, 2H), $2.43(\mathrm{~s}, 3 \mathrm{H}), 2.40-2.30(\mathrm{~m}, 1 \mathrm{H}), 2.04(\mathrm{dt}, J=11.9,3.5 \mathrm{~Hz}, 1 \mathrm{H})$, 1.99-1.89 (m, 1H), 1.19-1.10 (m, 1H), 1.07-0.96 (m, 21H). $\left.{ }^{13} \mathrm{C} \mathrm{NMR} \mathrm{(101} \mathrm{MHz,} \mathrm{CDCl}_{3}\right)$ $\delta 143.6,136.3,133.7,129.8,127.7,118.5,67.9,53.9,50.7,39.4,35.9,35.4,21.7,18.2$, 12.4. HRMS (ESI) calcd. for $\mathrm{C}_{24} \mathrm{H}_{39} \mathrm{NNaO}_{3} \mathrm{SSi}^{+}(\mathrm{M}+\mathrm{Na})^{+}$: 472.2312, Found: 472.2318 . 
<smiles>[3H]N1CC2=CC[C@H](OCC)[C@H](/C=C/C)[C@@H]2C1</smiles>

( \pm )-4-(Prop-1-en-1-yl)-2-tosyl-5-((triethylsilyl)oxy)-2,3,3a,4,5,6-hexahydro-1H-iso indole (3i). Reaction conditions: $\mathrm{Et}_{3} \mathrm{SiH}$ (2.0 equiv), $\mathrm{B}\left(\mathrm{C}_{6} \mathrm{~F}_{5}\right)_{3}(5 \mathrm{~mol} \%), \mathrm{DCE}$, $120{ }^{\circ} \mathrm{C}$. White solid (41 mg, 46\% yield), $E / Z=4.5: 1.0 .{ }^{1} \mathrm{H} \mathrm{NMR}\left(400 \mathrm{MHz}, \mathrm{CDCl}_{3}\right) \delta$ (E): $7.70(\mathrm{~d}, J=8.1 \mathrm{~Hz}, 2 \mathrm{H}), 7.32(\mathrm{~d}, J=8.1 \mathrm{~Hz}, 2 \mathrm{H}), 5.49-5.34(\mathrm{~m}, 1.85 \mathrm{H}), 5.26-5.18$ (m, 0.81H), $3.95(\mathrm{~d}, J=13.4 \mathrm{~Hz}, 1 \mathrm{H}), 3.75-3.56(\mathrm{~m}, 3 \mathrm{H}), 2.60-2.51(\mathrm{~m}, 1 \mathrm{H}), 2.47-2.29$ (m, 5H), 2.05-1.94 (m, 1H), $1.79(\mathrm{dd}, J=18.9,9.9 \mathrm{~Hz}, 1 \mathrm{H}), 1.67-1.64(\mathrm{~m}, 2.48 \mathrm{H})$, 0.95-0.87 (m, 9H), 0.57-0.48 (m, 6H). (Z) (distinguishable peaks): 5.64-5.53 (m, $0.18 \mathrm{H}), 5.14-5.03(\mathrm{~m}, 0.18 \mathrm{H}), 1.60-1.56(\mathrm{~m}, 0.54 \mathrm{H}) .{ }^{13} \mathrm{C} \mathrm{NMR}\left(101 \mathrm{MHz}, \mathrm{CDCl}_{3}\right) \delta$ 143.6, 135.6, 133.7, 131.5, 129.8, 127.7, 127.2, 118.0, 70.9, 53.5, 51.1, 49.5, 44.2, 35.9, 21.7, 18.2, 6.9, 5.0. HRMS (MALDI) calcd. for $\mathrm{C}_{24} \mathrm{H}_{37} \mathrm{NNaO}_{3} \mathrm{SSi}^{+}(\mathrm{M}+\mathrm{Na})^{+}$: 470.2156, Found: 470.2158 .

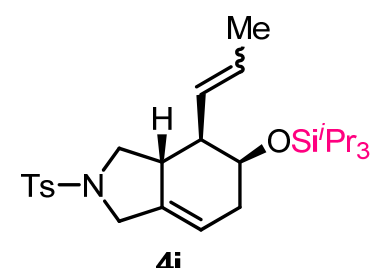

( \pm )-4-(Prop-1-en-1-yl)-2-tosyl-5-((triisopropylsilyl)oxy)-2,3,3a,4,5,6-hexahydro-1 H-isoindole (4i). Reaction conditions: ${ }^{i} \mathrm{Pr}_{3} \mathrm{SiH}$ (1.5 equiv), $\mathrm{B}\left(\mathrm{C}_{6} \mathrm{~F}_{5}\right)_{3}(5 \mathrm{~mol} \%)$, toluene, $110{ }^{\circ} \mathrm{C}$. White solid (66 mg, 67\% yield), $E / Z=5.5: 1.0 .{ }^{1} \mathrm{H}$ NMR $(400 \mathrm{MHz}$, $\left.\mathrm{CDCl}_{3}\right) \delta(E): 7.70(\mathrm{~d}, J=8.1 \mathrm{~Hz}, 2 \mathrm{H}), 7.31(\mathrm{~d}, J=8.1 \mathrm{~Hz}, 2 \mathrm{H}), 5.56-5.47(\mathrm{~m}, 1 \mathrm{H})$, 5.45-5.36 (m, 1H), $5.32(\mathrm{~s}, 1 \mathrm{H}), 4.08-4.00(\mathrm{~m}, 2 \mathrm{H}), 3.76-3.65(\mathrm{~m}, 2 \mathrm{H}), 2.74-2.63(\mathrm{~m}$, $1 \mathrm{H}), 2.62-2.51(\mathrm{~m}, 1 \mathrm{H}), 2.41(\mathrm{~s}, 3 \mathrm{H}), 2.26-2.08(\mathrm{~m}, 2 \mathrm{H}), 1.72-1.66(\mathrm{~m}, 1 \mathrm{H}), 1.66-1.61$ $(\mathrm{m}, 2.54 \mathrm{H}), 1.03-0.90(\mathrm{~m}, 21 \mathrm{H}) .(\mathrm{Z})$ (distinguishable peaks): $1.59-1.51(\mathrm{~m}, 0.43 \mathrm{H}) .{ }^{13} \mathrm{C}$ NMR $\left(101 \mathrm{MHz}, \mathrm{CDCl}_{3}\right) \delta 143.4,136.3,133.9,132.4,129.8,127.7,126.1,115.8,70.0$, 53.5, 51.5, 47.8, 38.1, 35.1, 21.6, 18.3, 18.2, 18.1, 12.8. HRMS (MALDI) calcd. for $\mathrm{C}_{27} \mathrm{H}_{43} \mathrm{NNaO}_{3} \mathrm{SSi}^{+}(\mathrm{M}+\mathrm{Na})^{+}:$512.2625, Found: 512.2630.<smiles>[M]C12CN([3H])CC1=CC[C@H](OCC)C2C</smiles>

3j

( \pm )-3a,4-Dimethyl-2-tosyl-5-((triethylsilyl)oxy)-2,3,3a,4,5,6-hexahydro-1H-isoindo le (3j). Reaction conditions: $\mathrm{Et}_{3} \mathrm{SiH}$ (1.5 equiv), $\mathrm{B}\left(\mathrm{C}_{6} \mathrm{~F}_{5}\right)_{3}(5 \mathrm{~mol} \%)$, toluene, $120{ }^{\circ} \mathrm{C}$. Colorless oil (47 mg, 54\% yield). ${ }^{1} \mathrm{H}$ NMR $\left(400 \mathrm{MHz}, \mathrm{CDCl}_{3}\right) \delta 7.69(\mathrm{~d}, J=8.1 \mathrm{~Hz}$, 2H), $7.31(\mathrm{~d}, J=8.1 \mathrm{~Hz}, 2 \mathrm{H}), 5.27(\mathrm{~s}, 1 \mathrm{H}), 4.07-3.98(\mathrm{~m}, 1 \mathrm{H}), 3.71-3.60(\mathrm{~m}, 2 \mathrm{H}), 3.42$ $(\mathrm{d}, J=8.9 \mathrm{~Hz}, 1 \mathrm{H}), 2.71(\mathrm{~d}, J=8.9 \mathrm{~Hz}, 1 \mathrm{H}), 2.42(\mathrm{~s}, 3 \mathrm{H}), 2.40-2.30(\mathrm{~m}, 1 \mathrm{H}), 1.99-1.88$ (m, 1H), 1.43-1.33 (m, 1H), 0.93 (t, $J=7.9 \mathrm{~Hz}, 12 \mathrm{H}), 0.84$ (s, 3H), 0.57 (q, $J=7.9 \mathrm{~Hz}$, 
$6 \mathrm{H}) .{ }^{13} \mathrm{C} \mathrm{NMR}\left(101 \mathrm{MHz}, \mathrm{CDCl}_{3}\right) \delta 143.5,140.4,134.1,129.8,127.5,117.4,69.5,61.4$, 50.5, 45.7, 43.6, 36.2, 21.7, 18.9, 12.7, 7.0, 5.1. HRMS (MALDI) calcd. for $\mathrm{C}_{23} \mathrm{H}_{37} \mathrm{NNaO}_{3} \mathrm{SSi}^{+} \quad(\mathrm{M}+\mathrm{Na})^{+}:$458.2156, Found: 458.2158. The relative stereochemistry of this compound was assigned by NOE experiment.

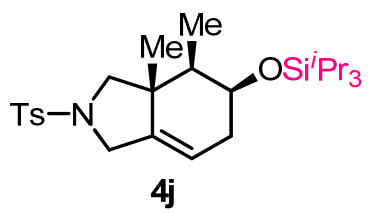

( \pm )-3a,4-Dimethyl-2-tosyl-5-((triisopropylsilyl)oxy)-2,3,3a,4,5,6-hexahydro-1H-iso indole (4j). Reaction conditions: ${ }^{i} \mathrm{Pr}_{3} \mathrm{SiH}$ (1.5 equiv), $\mathrm{B}\left(\mathrm{C}_{6} \mathrm{~F}_{5}\right)_{3}(10 \mathrm{~mol} \%)$, toluene, $130{ }^{\circ} \mathrm{C}$. White solid (34 mg, 36\% yield). ${ }^{1} \mathrm{H}$ NMR (400 MHz, $\left.\mathrm{CDCl}_{3}\right) \delta 7.71$ (d, $J=7.9$ $\mathrm{Hz}, 2 \mathrm{H}), 7.31(\mathrm{~d}, J=7.9 \mathrm{~Hz}, 2 \mathrm{H}), 5.31(\mathrm{~s}, 1 \mathrm{H}), 4.10-3.91(\mathrm{~m}, 2 \mathrm{H}), 3.75(\mathrm{~d}, J=12.5 \mathrm{~Hz}$, $1 \mathrm{H}), 3.37(\mathrm{~d}, J=8.8 \mathrm{~Hz}, 1 \mathrm{H}), 2.74(\mathrm{~d}, J=8.8 \mathrm{~Hz}, 1 \mathrm{H}), 2.42(\mathrm{~s}, 3 \mathrm{H}), 2.34-2.25(\mathrm{~m}, 1 \mathrm{H})$, $2.18(\mathrm{~d}, J=19.1 \mathrm{~Hz}, 1 \mathrm{H}), 1.40-1.33(\mathrm{~m}, 1 \mathrm{H}), 1.07-0.95(\mathrm{~m}, 27 \mathrm{H}) .{ }^{13} \mathrm{C} \mathrm{NMR}(101 \mathrm{MHz}$, $\left.\mathrm{CDCl}_{3}\right) \delta 143.3,140.3,134.7,129.7,127.5,116.0,70.6,62.6,51.0,42.9,41.3,35.3$, 21.7, 19.1, 18.4, 18.4, 14.4, 12.9. HRMS (MALDI) calcd. for $\mathrm{C}_{26} \mathrm{H}_{43} \mathrm{NNaO}_{3} \mathrm{SSi}^{+}$ $(\mathrm{M}+\mathrm{Na})^{+}: 500.2625$, Found: 500.2628 . The relative stereochemistry of this compound was assigned by NOE experiment.<smiles>[3H]N1CC2=CC[C@H](OCC)C(c3ccccc3)C2C1</smiles>

(士)-4-Phenyl-2-tosyl-5-((triethylsilyl)oxy)-2,3,3a,4,5,6-hexahydro-1H-isoindole

(3k). Reaction conditions: $\mathrm{Et}_{3} \mathrm{SiH}\left(1.5\right.$ equiv), $\mathrm{B}\left(\mathrm{C}_{6} \mathrm{~F}_{5}\right) 3(5 \mathrm{~mol} \%)$, DCE, $90{ }^{\circ} \mathrm{C}$. White solid (60 mg, 62\% yield). ${ }^{1} \mathrm{H}$ NMR (400 MHz, $\left.\mathrm{CDCl}_{3}\right) \delta 7.64(\mathrm{~d}, J=8.2 \mathrm{~Hz}, 2 \mathrm{H}), 7.31$ $(\mathrm{d}, J=8.2 \mathrm{~Hz}, 2 \mathrm{H}), 7.27(\mathrm{~d}, J=6.5 \mathrm{~Hz}, 2 \mathrm{H}), 7.24-7.18(\mathrm{~m}, 1 \mathrm{H}), 7.13-7.09(\mathrm{~m}, 2 \mathrm{H})$, 5.49-5.45 (m, 1H), 4.08-4.01 (m, 1H), 3.98-3.90 (m, 1H), 3.73-3.66 (m, 1H), $3.46(\mathrm{dd}, J$ $=9.0,8.0 \mathrm{~Hz}, 1 \mathrm{H}), 2.95-2.84(\mathrm{~m}, 1 \mathrm{H}), 2.56-2.46(\mathrm{~m}, 2 \mathrm{H}), 2.44(\mathrm{~s}, 3 \mathrm{H}), 2.33(\mathrm{t}, J=10.6$ $\mathrm{Hz}, 1 \mathrm{H}), 2.19-2.07(\mathrm{~m}, 1 \mathrm{H}), 0.67(\mathrm{t}, J=7.9 \mathrm{~Hz}, 9 \mathrm{H}), 0.34-0.12(\mathrm{~m}, 6 \mathrm{H}) .{ }^{13} \mathrm{C}$ NMR $(101$ $\left.\mathrm{MHz}, \mathrm{CDCl}_{3}\right) \delta 143.6,141.0,136.2,133.6,129.8,128.4,128.3,127.7,126.9,118.0$, 71.7, 53.3, 52.4, 51.1, 45.3, 36.9, 21.7, 6.7, 4.7. HRMS (MALDI) calcd. for $\mathrm{C}_{27} \mathrm{H}_{37} \mathrm{NNaO}_{3} \mathrm{SSi}^{+}(\mathrm{M}+\mathrm{Na})^{+}: 506.2156$, Found: 506.2160. The structure and relative stereochemistry of this compound was determined by X-ray crystallography and NOE experiment.<smiles>[B]N1CC2=CCC(O[As])C(c3ccccc3)C2C1</smiles>

( \pm )-4-Phenyl-2-tosyl-5-((triisopropylsilyl)oxy)-2,3,3a,4,5,6-hexahydro-1H-isoindol e (4k). Reaction conditions: ${ }^{i} \mathrm{Pr}_{3} \mathrm{SiH}\left(1.5\right.$ equiv), $\mathrm{B}\left(\mathrm{C}_{6} \mathrm{~F}_{5}\right)_{3}(5 \mathrm{~mol} \%)$, toluene, $95{ }^{\circ} \mathrm{C}$. Colorless oil (58 mg, 55\% yield). ${ }^{1} \mathrm{H}$ NMR (400 MHz, $\left.\mathrm{CDCl}_{3}\right) \delta 7.68(\mathrm{~d}, J=8.2 \mathrm{~Hz}$, $2 \mathrm{H}), 7.31(\mathrm{~d}, J=8.2 \mathrm{~Hz}, 2 \mathrm{H}), 7.29-7.25(\mathrm{~m}, 2 \mathrm{H}), 7.24-7.16(\mathrm{~m}, 3 \mathrm{H}), 5.43(\mathrm{~s}, 1 \mathrm{H})$, 4.21-4.06 (m, 2H), 3.78-3.70 (m, 2H), 3.34-3.23 (m, 1H), 2.50 (dd, $J=10.9,9.7 \mathrm{~Hz}$, 1H), 2.45-2.33 (m, 5H), 2.31-2.22 (m, 1H), 0.87-0.72 (m, 21H). ${ }^{13} \mathrm{C}$ NMR (101 MHz, 
$\left.\mathrm{CDCl}_{3}\right) \delta 143.5,141.5,136.8,133.8,129.8,128.4,128.3,127.8,126.9,115.9,71.3$, 53.3, 51.5, 49.9, 37.0, 35.9, 21.7, 18.1, 17.9, 13.0. HRMS (MALDI) calcd. for $\mathrm{C}_{30} \mathrm{H}_{43} \mathrm{NNaO}_{3} \mathrm{SSi}^{+} \quad(\mathrm{M}+\mathrm{Na})^{+}:$548.2625, Found: 548.2630. The relative stereochemistry of this compound was assigned by NOE experiment.<smiles>[3H]N1CC2=CC[C@H](OCC)[C@H](c3ccc(Oc4ccccc4)cc3)[C@H]2C1</smiles>

31

( \pm )-4-(4-Phenoxyphenyl)-2-tosyl-5-((triethylsilyl)oxy)-2,3,3a,4,5,6-hexahydro-1Hisoindole (3l). Reaction conditions: $\mathrm{Et}_{3} \mathrm{SiH}$ (1.5 equiv), $\mathrm{B}\left(\mathrm{C}_{6} \mathrm{~F}_{5}\right)_{3}(5 \mathrm{~mol} \%), \mathrm{DCE}$, $95{ }^{\circ} \mathrm{C}$. White solid (59 mg, 51\% yield). ${ }^{1} \mathrm{H}$ NMR (400 $\left.\mathrm{MHz}, \mathrm{CDCl}_{3}\right) \delta 7.71-7.63(\mathrm{~m}$, 2H), 7.36-7.30 (m, 4H), 7.12-7.06 (m, 3H), 7.01-6.93 (m, 4H), $5.47(\mathrm{~s}, 1 \mathrm{H}), 4.05(\mathrm{~d}, J=$ $13.5 \mathrm{~Hz}, 1 \mathrm{H}), 3.95-3.86(\mathrm{~m}, 1 \mathrm{H}), 3.69(\mathrm{~d}, J=13.5 \mathrm{~Hz}, 1 \mathrm{H}), 3.50(\mathrm{t}, J=8.4 \mathrm{~Hz}, 1 \mathrm{H})$, 2.95-2.83 (m, 1H), 2.58-2.40 (m, 5H), $2.33(\mathrm{t}, J=10.5 \mathrm{~Hz}, 1 \mathrm{H}), 2.20-2.08(\mathrm{~m}, 1 \mathrm{H}), 0.73$ $(\mathrm{t}, J=7.9 \mathrm{~Hz}, 9 \mathrm{H}), 0.38-0.20(\mathrm{~m}, 6 \mathrm{H}) .{ }^{13} \mathrm{C} \mathrm{NMR}\left(101 \mathrm{MHz}, \mathrm{CDCl}_{3}\right) \delta 157.8,156.0$, 143.7, 136.2, 136.1, 133.5, 129.8, 129.8, 129.5, 127.7, 123.0, 119.3, 118.5, 118.0, 71.8, 53.3, 51.6, 51.1, 45.3, 36.8, 21.7, 6.8, 4.8. HRMS (ESI) calcd. for $\mathrm{C}_{33} \mathrm{H}_{41} \mathrm{NNaO}_{4} \mathrm{SSi}^{+}$ $(\mathrm{M}+\mathrm{Na})^{+}:$598.2418, Found: 598.2422.<smiles>[GaH2]O[C@H]1CC=C2CN([AsH3-])C[C@@H]2[C@H]1c1ccc(Oc2ccccc2)cc1</smiles>

( \pm )-4-(4-Phenoxyphenyl)-2-tosyl-5-((triisopropylsilyl)oxy)-2,3,3a,4,5,6-hexahydro -1H-isoindole (4l). Reaction conditions: ${ }^{i} \mathrm{Pr}_{3} \mathrm{SiH}$ (1.5 equiv), $\mathrm{B}\left(\mathrm{C}_{6} \mathrm{~F}_{5}\right)_{3}(5 \mathrm{~mol} \%)$, toluene, $95{ }^{\circ} \mathrm{C}$. Colorless oil $\left(68 \mathrm{mg}, 55 \%\right.$ yield). ${ }^{1} \mathrm{H}$ NMR $\left(400 \mathrm{MHz}, \mathrm{CDCl}_{3}\right) \delta$ 7.75-7.64 (m, 2H), 7.37-7.28 (m, 4H), 7.20-7.12 (m, 2H), 7.12-7.05 (m, 1H), 7.00-6.93 (m, 4H), $5.44(\mathrm{~s}, 1 \mathrm{H}), 4.24-4.08(\mathrm{~m}, 2 \mathrm{H}), 3.80-3.70(\mathrm{~m}, 2 \mathrm{H}), 3.31-3.17(\mathrm{~m}, 1 \mathrm{H}), 2.54(\mathrm{t}$, $J=10.3 \mathrm{~Hz}, 1 \mathrm{H}), 2.46-2.33(\mathrm{~m}, 5 \mathrm{H}), 2.32-2.23(\mathrm{~m}, 1 \mathrm{H}), 0.91-0.82(\mathrm{~m}, 21 \mathrm{H}) .{ }^{13} \mathrm{C} \mathrm{NMR}$ $\left(101 \mathrm{MHz}, \mathrm{CDCl}_{3}\right) \delta 157.9,155.8,143.6,136.7,133.6,129.8,129.8,129.6,127.7$, 123.0, 119.3, 118.4, 115.9, 71.3, 53.3, 51.4, 49.2, 37.2, 35.8, 21.7, 18.2, 18.0, 13.0. HRMS (ESI) calcd. for $\mathrm{C}_{36} \mathrm{H}_{47} \mathrm{NNaO}_{4} \mathrm{SSi}^{+}(\mathrm{M}+\mathrm{Na})^{+}$: 640.2887, Found: 640.2892 .<smiles>[3H]N1C[C@@H]2CC[C@H](OCC)[C@H](c3ccccc3)[C@]2(c2ccc([N+](=O)[O-])cc2)C1</smiles> 
( \pm )-4-(p-Tolyl)-2-tosyl-5-((triethylsilyl)oxy)-2,3,3a,4,5,6-hexahydro-1H-isoindole

(3m). Reaction conditions: $\mathrm{Et}_{3} \mathrm{SiH}$ (1.5 equiv), $\mathrm{B}\left(\mathrm{C}_{6} \mathrm{~F}_{5}\right)_{3}(5 \mathrm{~mol} \%), \mathrm{DCE}, 95{ }^{\circ} \mathrm{C}$. White solid (56 mg, 56\% yield). ${ }^{1} \mathrm{H}$ NMR (400 MHz, $\left.\mathrm{CDCl}_{3}\right) \delta 7.64(\mathrm{~d}, J=8.1 \mathrm{~Hz}$, 2H), $7.30(\mathrm{~d}, J=8.1 \mathrm{~Hz}, 2 \mathrm{H}), 7.10-7.05(\mathrm{~m}, 2 \mathrm{H}), 7.02-6.96(\mathrm{~m}, 2 \mathrm{H}), 5.45(\mathrm{~s}, 1 \mathrm{H}), 4.03$ $(\mathrm{d}, J=13.5 \mathrm{~Hz}, 1 \mathrm{H}), 3.96-3.86(\mathrm{~m}, 1 \mathrm{H}), 3.69(\mathrm{~d}, J=13.5 \mathrm{~Hz}, 1 \mathrm{H}), 3.46(\mathrm{t}, J=8.5 \mathrm{~Hz}$, $1 \mathrm{H}), 2.92-2.80(\mathrm{~m}, 1 \mathrm{H}), 2.55-2.49(\mathrm{~m}, 1 \mathrm{H}), 2.48-2.38(\mathrm{~m}, 4 \mathrm{H}), 2.35-2.24(\mathrm{~m}, 4 \mathrm{H})$, 2.19-2.06 (m, 1H), $0.67(\mathrm{t}, J=7.9 \mathrm{~Hz}, 9 \mathrm{H}), 0.35-0.15(\mathrm{~m}, 6 \mathrm{H}) .{ }^{13} \mathrm{C} \mathrm{NMR}(101 \mathrm{MHz}$, $\left.\mathrm{CDCl}_{3}\right) \delta 143.6,137.9,136.3,136.2,133.6,129.8,129.0,128.1,127.7,117.9,71.7$, 53.3, 51.9, 51.1, 45.3, 36.8, 21.7, 21.1, 6.7, 4.7. HRMS (MALDI) calcd. for $\mathrm{C}_{28} \mathrm{H}_{39} \mathrm{NNaO}_{3} \mathrm{SSi}^{+}(\mathrm{M}+\mathrm{Na})^{+}: 520.2312$, Found: 520.2316 .

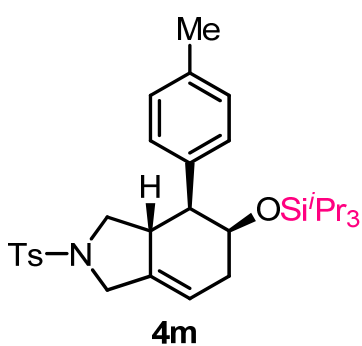

( \pm )-4-(p-Tolyl)-2-tosyl-5-((triisopropylsilyl)oxy)-2,3,3a,4,5,6-hexahydro-1H-isoind ole (4m). Reaction conditions: ${ }^{i} \mathrm{Pr}_{3} \mathrm{SiH}$ (1.5 equiv), $\mathrm{B}\left(\mathrm{C}_{6} \mathrm{~F}_{5}\right)_{3}(5 \mathrm{~mol} \%)$, toluene, $95{ }^{\circ} \mathrm{C}$ White solid (65 mg, 60\% yield). ${ }^{1} \mathrm{H}$ NMR (400 MHz, $\left.\mathrm{CDCl}_{3}\right) \delta 7.68(\mathrm{~d}, J=8.1 \mathrm{~Hz}$, $2 \mathrm{H}), 7.31(\mathrm{~d}, J=8.1 \mathrm{~Hz}, 2 \mathrm{H}), 7.10-7.05(\mathrm{~m}, 4 \mathrm{H}), 5.42(\mathrm{~s}, 1 \mathrm{H}), 4.20-4.10(\mathrm{~m}, 2 \mathrm{H})$, 3.78-3.68 (m, 2H), 3.31-3.20 (m, 1H), 2.49 (dd, $J=10.9,9.8 \mathrm{~Hz}, 1 \mathrm{H}), 2.42(\mathrm{~s}, 3 \mathrm{H})$, 2.38-2.21 (m, 6H), 0.88-0.79 (m, 21H). ${ }^{13} \mathrm{C} \mathrm{NMR}\left(101 \mathrm{MHz}, \mathrm{CDCl}_{3}\right) \delta$ 143.5, 138.4, 136.8, 136.3, 133.6, 129.8, 128.9, 128.2, 127.7, 115.8, 71.3, 53.3, 51.5, 49.5, 37.1, 35.9, 21.7, 21.1, 18.1, 17.9, 13.0. HRMS (MALDI) calcd. for $\mathrm{C}_{31} \mathrm{H}_{45} \mathrm{NNaO}_{3} \mathrm{SSi}^{+}(\mathrm{M}+\mathrm{Na})^{+}$: 562.2782, Found: 562.2785 .

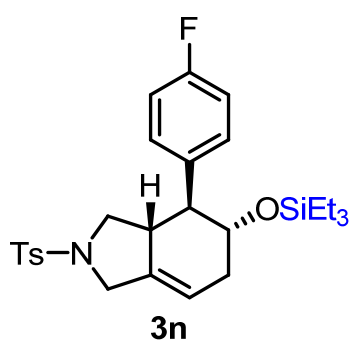

( \pm )-4-(4-Fluorophenyl)-2-tosyl-5-((triethylsilyl)oxy)-2,3,3a,4,5,6-hexahydro-1H-is

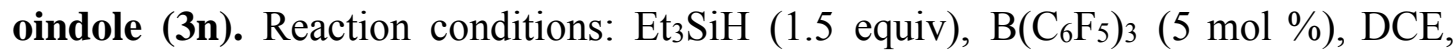
$90{ }^{\circ} \mathrm{C}$. White solid (67 mg, 67\% yield). ${ }^{1} \mathrm{H}$ NMR (400 MHz, $\left.\mathrm{CDCl}_{3}\right) \delta 7.64(\mathrm{~d}, J=8.1$ $\mathrm{Hz}, 2 \mathrm{H}), 7.31(\mathrm{~d}, J=8.1 \mathrm{~Hz}, 2 \mathrm{H}), 7.11-7.04(\mathrm{~m}, 2 \mathrm{H}), 7.01-6.95(\mathrm{~m}, 2 \mathrm{H}), 5.46(\mathrm{~s}, 1 \mathrm{H})$, 4.03 (d, $J=13.5 \mathrm{~Hz}, 1 \mathrm{H}), 3.88$ (ddd, $J=10.0,8.9,6.2 \mathrm{~Hz}, 1 \mathrm{H}), 3.68$ (d, $J=13.5 \mathrm{~Hz}$, $1 \mathrm{H}), 3.44(\mathrm{dd}, J=9.0,7.9 \mathrm{~Hz}, 1 \mathrm{H}), 2.90-2.79(\mathrm{~m}, 1 \mathrm{H}), 2.54-2.40(\mathrm{~m}, 5 \mathrm{H}), 2.32(\mathrm{t}, J$ $=10.6 \mathrm{~Hz}, 1 \mathrm{H}), 2.19-2.06(\mathrm{~m}, 1 \mathrm{H}), 0.68(\mathrm{t}, J=7.9 \mathrm{~Hz}, 9 \mathrm{H}), 0.34-0.15(\mathrm{~m}, 6 \mathrm{H}) .{ }^{13} \mathrm{C}$ NMR $\left(101 \mathrm{MHz}, \mathrm{CDCl}_{3}\right) \delta 162.0(\mathrm{~d}, J=244.4 \mathrm{~Hz}), 143.7,136.8(\mathrm{~d}, J=3.1 \mathrm{~Hz})$, 136.0, 133.5, 129.8, $129.6(\mathrm{~d}, J=7.9 \mathrm{~Hz}), 127.7,118.0,115.2(\mathrm{~d}, J=21.1 \mathrm{~Hz}), 71.7$, 53.2, 51.6, 51.0, 45.3, 36.8. 21.7, 6.7, 4.7. HRMS (MALDI) calcd. for $\mathrm{C}_{27} \mathrm{H}_{36} \mathrm{FNNaO}_{3} \mathrm{SSi}^{+}(\mathrm{M}+\mathrm{Na})^{+}:$524.2061, Found: 524.2065. 
<smiles></smiles>

( \pm )-4-(4-Fluorophenyl)-2-tosyl-5-((triisopropylsilyl)oxy)-2,3,3a,4,5,6-hexahydro-1 H-isoindole (4n). Reaction conditions: ${ }^{i} \mathrm{Pr}_{3} \mathrm{SiH}$ (1.5 equiv), $\mathrm{B}\left(\mathrm{C}_{6} \mathrm{~F}_{5}\right)_{3}$ (5 mol \%), toluene, $95{ }^{\circ} \mathrm{C}$. White solid ( $75 \mathrm{mg}, 69 \%$ yield). ${ }^{1} \mathrm{H}$ NMR $\left(400 \mathrm{MHz}, \mathrm{CDCl}_{3}\right) \delta 7.67$ (d, $J=8.1 \mathrm{~Hz}, 2 \mathrm{H}), 7.31$ (d, $J=8.1 \mathrm{~Hz}, 2 \mathrm{H}), 7.18-7.12(\mathrm{~m}, 2 \mathrm{H}), 7.00-6.93(\mathrm{~m}, 2 \mathrm{H}), 5.43$ (s, 1H), 4.18-4.02 (m, 2H), $3.73(\mathrm{~d}, J=13.7 \mathrm{~Hz}, 1 \mathrm{H}), 3.70-3.64(\mathrm{~m}, 1 \mathrm{H}), 3.27-3.15(\mathrm{~m}$, 1H), 2.51-2.45 (m, 1H), $2.42(\mathrm{~s}, 3 \mathrm{H}), 2.39-2.21(\mathrm{~m}, 3 \mathrm{H}), 0.90-0.72(\mathrm{~m}, 21 \mathrm{H}) .{ }^{13} \mathrm{C} \mathrm{NMR}$ $\left(101 \mathrm{MHz}, \mathrm{CDCl}_{3}\right) \delta 161.9(\mathrm{~d}, J=244.7 \mathrm{~Hz}), 143.6,137.2(\mathrm{~d}, J=3.1 \mathrm{~Hz}), 136.5,133.6$, $129.8,129.7$ (d, $J=7.9 \mathrm{~Hz}), 127.7,115.9,115.0$ (d, $J=21.0 \mathrm{~Hz}), 71.1,53.1,51.4,49.2$, 37.3, 35.8, 21.6, 18.1, 17.9, 12.9. HRMS (MALDI) calcd. for $\mathrm{C}_{30} \mathrm{H}_{42} \mathrm{FNNaO}_{3} \mathrm{SSi}^{+}$ $(\mathrm{M}+\mathrm{Na})^{+}: 566.2531$, Found: 566.2536 . The structure and relative stereochemistry of this compound was determined by X-ray crystallography.<smiles>[3H]N1CC2=CC[C@H](OCC)[C@H](CC)[C@]2(c2ccc(Br)cc2)C1</smiles>

( \pm )-4-(4-Bromophenyl)-2-tosyl-5-((triethylsilyl)oxy)-2,3,3a,4,5,6-hexahydro-1H-is oindole (3o). Reaction conditions: $\mathrm{Et}_{3} \mathrm{SiH}$ (1.5 equiv), $\mathrm{B}\left(\mathrm{C}_{6} \mathrm{~F}_{5}\right)_{3}(5 \mathrm{~mol} \%), \mathrm{DCE}$, $90{ }^{\circ} \mathrm{C}$. White solid (74 mg, 66\% yield). ${ }^{1} \mathrm{H}$ NMR (400 MHz, $\left.\mathrm{CDCl}_{3}\right) \delta 7.64$ (d, $J=8.1$ $\mathrm{Hz}, 2 \mathrm{H}), 7.41$ (d, $J=8.4 \mathrm{~Hz}, 2 \mathrm{H}), 7.31$ (d, $J=8.1 \mathrm{~Hz}, 2 \mathrm{H}), 7.00$ (d, $J=8.4 \mathrm{~Hz}, 2 \mathrm{H})$, 5.49-5.44 (m, 1H), $4.03(\mathrm{~d}, J=13.6 \mathrm{~Hz}, 1 \mathrm{H}), 3.93-3.85(\mathrm{~m}, 1 \mathrm{H}), 3.69(\mathrm{~d}, J=13.6 \mathrm{~Hz}$, $1 \mathrm{H}), 3.42(\mathrm{dd}, J=9.1,7.9 \mathrm{~Hz}, 1 \mathrm{H}), 2.88-2.78(\mathrm{~m}, 1 \mathrm{H}), 2.53-2.42(\mathrm{~m}, 5 \mathrm{H}), 2.30(\mathrm{t}, J=$ $10.6 \mathrm{~Hz}, 1 \mathrm{H}), 2.18-2.06(\mathrm{~m}, 1 \mathrm{H}), 0.68(\mathrm{t}, J=7.9 \mathrm{~Hz}, 9 \mathrm{H}), 0.35-0.18(\mathrm{~m}, 6 \mathrm{H}) .{ }^{13} \mathrm{C} \mathrm{NMR}$ $\left(101 \mathrm{MHz}, \mathrm{CDCl}_{3}\right) \delta 143.7,140.2,136.0,133.6,131.5,130.0,129.9,127.7,120.6$, 118.1, 71.6, 53.1, 51.9, 51.0, 45.1, 36.8, 21.7, 6.7, 4.8. HRMS (MALDI) calcd. for $\mathrm{C}_{27} \mathrm{H}_{36} \mathrm{BrNNaO}_{3} \mathrm{SSi}^{+}(\mathrm{M}+\mathrm{Na})^{+}:$584.1261, Found: 584.1265.

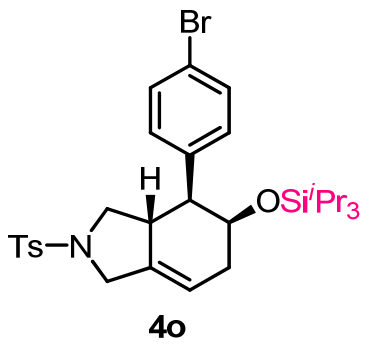

( \pm )-4-(4-Bromophenyl)-2-tosyl-5-((triisopropylsilyl)oxy)-2,3,3a,4,5,6-hexahydro-1 H-isoindole (4o). Reaction conditions: ${ }^{i} \mathrm{Pr}_{3} \mathrm{SiH}$ (1.5 equiv), $\mathrm{B}\left(\mathrm{C}_{6} \mathrm{~F}_{5}\right)_{3}(5 \mathrm{~mol} \%$ ), 
toluene, $95{ }^{\circ} \mathrm{C}$. White solid ( $83 \mathrm{mg}, 69 \%$ yield). ${ }^{1} \mathrm{H} \mathrm{NMR}\left(400 \mathrm{MHz}, \mathrm{CDCl}_{3}\right) \delta 7.67$ (d, $J=8.2 \mathrm{~Hz}, 2 \mathrm{H}), 7.43-7.37(\mathrm{~m}, 2 \mathrm{H}), 7.31(\mathrm{~d}, J=8.0 \mathrm{~Hz}, 2 \mathrm{H}), 7.09-7.03(\mathrm{~m}, 2 \mathrm{H}), 5.43(\mathrm{~s}$, $1 \mathrm{H}), 4.19-4.02(\mathrm{~m}, 2 \mathrm{H}), 3.77-3.70(\mathrm{~m}, 1 \mathrm{H}), 3.66(\mathrm{dd}, J=9.3,7.9 \mathrm{~Hz}, 1 \mathrm{H}), 3.26-3.13(\mathrm{~m}$, $1 \mathrm{H}), 2.51-2.43(\mathrm{~m}, 1 \mathrm{H}), 2.42(\mathrm{~s}, 3 \mathrm{H}), 2.38-2.21(\mathrm{~m}, 3 \mathrm{H}), 0.89-0.73(\mathrm{~m}, 21 \mathrm{H}) .{ }^{13} \mathrm{C}$ NMR $\left(101 \mathrm{MHz}, \mathrm{CDCl}_{3}\right) \delta 143.6,140.6,136.5,133.6,131.3,130.1,129.8,127.7,120.7$, 115.9, 71.0, 53.0, 51.4, 49.4, 37.1, 35.8, 21.7, 18.1, 17.9, 13.0. HRMS (MALDI) calcd. for $\mathrm{C}_{30} \mathrm{H}_{42} \mathrm{BrNNaO}_{3} \mathrm{SSi}^{+}(\mathrm{M}+\mathrm{Na})^{+}$: 626.1730, Found: 626.1735 .<smiles>[3H]N1CC2=CC[C@H](OCC)[C@H](C)[C@]2(c2ccc(C(F)(F)F)cc2)C1</smiles>

( \pm )-2-Tosyl-5-((triethylsilyl)oxy)-4-(4-(trifluoromethyl)phenyl)-2,3,3a,4,5,6-hexah ydro-1H-isoindole (3p). Reaction conditions: $\mathrm{Et}_{3} \mathrm{SiH}$ (1.5 equiv), $\mathrm{B}\left(\mathrm{C}_{6} \mathrm{~F}_{5}\right)_{3}(5 \mathrm{~mol} \%)$, DCE, $75{ }^{\circ} \mathrm{C}$. White solid (68 mg, 62\% yield). ${ }^{1} \mathrm{H}$ NMR $\left(400 \mathrm{MHz}, \mathrm{CDCl}_{3}\right) \delta 7.65$ (d, $J$ $=8.2 \mathrm{~Hz}, 2 \mathrm{H}), 7.56(\mathrm{~d}, J=8.1 \mathrm{~Hz}, 2 \mathrm{H}), 7.32(\mathrm{~d}, J=8.0 \mathrm{~Hz}, 2 \mathrm{H}), 7.25(\mathrm{~d}, J=7.5 \mathrm{~Hz}, 2 \mathrm{H})$, 5.52-5.47 (m, 1H), $4.04(\mathrm{~d}, J=13.6 \mathrm{~Hz}, 1 \mathrm{H}), 3.96(\mathrm{td}, J=9.0,6.2 \mathrm{~Hz}, 1 \mathrm{H}), 3.70(\mathrm{~d}, J=$ $13.6 \mathrm{~Hz}, 1 \mathrm{H}), 3.42(\mathrm{dd}, J=9.0,8.0 \mathrm{~Hz}, 1 \mathrm{H}), 2.97-2.85(\mathrm{~m}, 1 \mathrm{H}), 2.55-2.48(\mathrm{~m}, 2 \mathrm{H})$, 2.45-2.39 (m, 4H), 2.22- $2.09(\mathrm{~m}, 1 \mathrm{H}), 0.65(\mathrm{t}, J=7.9 \mathrm{~Hz}, 9 \mathrm{H}), 0.35-0.13(\mathrm{~m}, 6 \mathrm{H}) .{ }^{13} \mathrm{C}$ NMR $\left(101 \mathrm{MHz}, \mathrm{CDCl}_{3}\right) \delta 145.4(\mathrm{q}, J=1.2 \mathrm{~Hz}), 143.7,135.9,133.5,129.9,129.4(\mathrm{q}, J$ $=32.3), 128.6,127.7,125.3(\mathrm{q}, J=3.8), 124.3(\mathrm{q}, J=271.9 \mathrm{~Hz}), 118.1,71.6,53.0,52.3$, 51.0, 45.1, 36.7, 21.7, 6.6, 4.7. HRMS (MALDI) calcd. for $\mathrm{C}_{28} \mathrm{H}_{36} \mathrm{~F}_{3} \mathrm{NNaO}_{3} \mathrm{SSi}^{+}$ $(\mathrm{M}+\mathrm{Na})^{+}:$574.2029, Found: 574.2033.

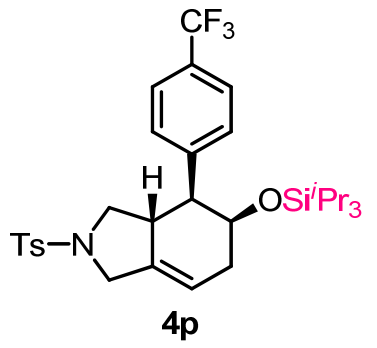

( \pm )-2-Tosyl-4-(4-(trifluoromethyl)phenyl)-5-((triisopropylsilyl)oxy)-2,3,3a,4,5,6-h exahydro-1H-isoindole (4p). Reaction conditions: ${ }^{i} \mathrm{Pr}_{3} \mathrm{SiH}$ (1.5 equiv), $\mathrm{B}\left(\mathrm{C}_{6} \mathrm{~F}_{5}\right)_{3}$ (5 mol \%), toluene, $95{ }^{\circ} \mathrm{C}$. White solid (70 mg, 59\% yield). ${ }^{1} \mathrm{H}$ NMR (400 MHz, $\mathrm{CDCl}_{3}$ ) $\delta 7.68(\mathrm{~d}, J=8.0 \mathrm{~Hz}, 2 \mathrm{H}), 7.55(\mathrm{~d}, J=7.9 \mathrm{~Hz}, 2 \mathrm{H}), 7.32(\mathrm{~d}, J=7.9 \mathrm{~Hz}, 4 \mathrm{H}), 5.50-5.43$ (m, 1H), 4.23-4.06 (m, 2H), $3.76(\mathrm{~d}, J=13.7 \mathrm{~Hz}, 1 \mathrm{H}), 3.69(\mathrm{dd}, J=9.3,8.0 \mathrm{~Hz}, 1 \mathrm{H})$, 3.34-3.22 (m, 1H), 2.54-2.47 (m, 1H), 2.45-2.39 (m, 5H), 2.33-2.24 (m, 1H), 0.88-0.74 $(\mathrm{m}, 21 \mathrm{H}) .{ }^{13} \mathrm{C} \mathrm{NMR}\left(101 \mathrm{MHz}, \mathrm{CDCl}_{3}\right) \delta 145.7,143.7,136.4,133.5,129.8,129.3$ (q, $J$ $=32.4 \mathrm{~Hz}), 128.7,127.7,125.2(\mathrm{q}, J=3.6 \mathrm{~Hz}), 124.3$ (q, $J=271.9 \mathrm{~Hz}), 116.0,71.0$, 53.0, 51.3, 49.8, 36.9, 35.8, 21.7, 18.0, 17.8, 12.9. HRMS (MALDI) calcd. for $\mathrm{C}_{31} \mathrm{H}_{42} \mathrm{~F}_{3} \mathrm{NNaO}_{3} \mathrm{SSi}^{+}(\mathrm{M}+\mathrm{Na})^{+}$: 616.2499, Found: 616.2502. 


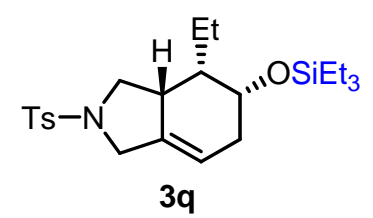

( \pm )-4-Ethyl-2-tosyl-5-((triethylsilyl)oxy)-2,3,3a,4,5,6-hexahydro-1H-isoindole (3q). Reaction conditions: $\mathrm{Et}_{3} \mathrm{SiH}$ (2.0 equiv), $\mathrm{B}\left(\mathrm{C}_{6} \mathrm{~F}_{5}\right)_{3}(5 \mathrm{~mol} \%), \mathrm{DCE}, 120{ }^{\circ} \mathrm{C}$. White solid (33 mg, 38\% yield). ${ }^{1} \mathrm{H}$ NMR (400 MHz, $\left.\mathrm{CDCl}_{3}\right) \delta 7.70(\mathrm{~d}, J=8.2 \mathrm{~Hz}, 2 \mathrm{H}), 7.32$ $(\mathrm{d}, J=8.2 \mathrm{~Hz}, 2 \mathrm{H}), 5.37(\mathrm{~s}, 1 \mathrm{H}), 3.92(\mathrm{~d}, J=13.5 \mathrm{~Hz}, 1 \mathrm{H}), 3.87-3.80(\mathrm{~m}, 1 \mathrm{H}), 3.64-3.58$ (m, 1H), $3.55(\mathrm{~d}, J=13.5 \mathrm{~Hz}, 1 \mathrm{H}), 2.82(\mathrm{dd}, J=11.2,8.9 \mathrm{~Hz}, 1 \mathrm{H}), 2.74-2.65(\mathrm{~m}, 1 \mathrm{H})$, $2.43(\mathrm{~s}, 3 \mathrm{H}), 2.12-2.01(\mathrm{~m}, 1 \mathrm{H}), 1.99-1.87(\mathrm{~m}, 1 \mathrm{H}), 1.71-1.64(\mathrm{~m}, 1 \mathrm{H}), 1.54-1.47$ (m, $1 \mathrm{H}), 0.93-0.82(\mathrm{~m}, 13 \mathrm{H}), 0.52(\mathrm{q}, J=7.9 \mathrm{~Hz}, 6 \mathrm{H}) .{ }^{13} \mathrm{C} \mathrm{NMR}\left(101 \mathrm{MHz}, \mathrm{CDCl}_{3}\right) \delta 143.6$, 135.1, 133.6, 129.8, 127.7, 117.6, 70.2, 50.6, 50.5, 43.1, 42.5, 31.4, 21.7, 16.0, 14.8, 7.0, 5.0. HRMS (MALDI) calcd. for $\mathrm{C}_{23} \mathrm{H}_{37} \mathrm{NNaO}_{3} \mathrm{SSi}^{+}(\mathrm{M}+\mathrm{Na})^{+}:$458.2156, Found: 458.2160. The relative stereochemistry of this compound was assigned by NOE experiment.

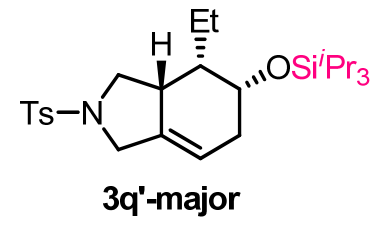

( \pm )-4-Ethyl-2-tosyl-5-((triisopropylsilyl)oxy)-2,3,3a,4,5,6-hexahydro-1H-isoindole (3q'). Reaction conditions: ${ }^{i} \mathrm{Pr}_{3} \mathrm{SiH}$ (1.5 equiv), $\mathrm{B}\left(\mathrm{C}_{6} \mathrm{~F}_{5}\right)_{3}(5 \mathrm{~mol} \%)$, toluene, $115^{\circ} \mathrm{C}$. White solid (44 mg, 46\% yield), $\mathrm{dr}=4.1: 1.0$. Major: ${ }^{1} \mathrm{H} \mathrm{NMR}\left(400 \mathrm{MHz}, \mathrm{CDCl}_{3}\right) \delta$ $7.70(\mathrm{~d}, J=8.2 \mathrm{~Hz}, 2 \mathrm{H}), 7.32(\mathrm{~d}, J=8.2 \mathrm{~Hz}, 2 \mathrm{H}), 5.37(\mathrm{~s}, 1 \mathrm{H}), 3.97-3.87(\mathrm{~m}, 2 \mathrm{H})$, 3.67-3.60 (m, 1H), $3.55(\mathrm{~d}, J=13.6 \mathrm{~Hz}, 1 \mathrm{H}), 2.86-2.77(\mathrm{~m}, 1 \mathrm{H}), 2.75-2.66(\mathrm{~m}, 1 \mathrm{H})$, $2.43(\mathrm{~s}, 3 \mathrm{H}), 2.18-2.08(\mathrm{~m}, 1 \mathrm{H}), 1.98-1.87(\mathrm{~m}, 1 \mathrm{H}), 1.79-1.73(\mathrm{~m}, 1 \mathrm{H}), 1.60-1.51(\mathrm{~m}$, $1 \mathrm{H}), 1.05-0.97(\mathrm{~m}, 21 \mathrm{H}), 0.88-0.83(\mathrm{~m}, 4 \mathrm{H}) .{ }^{13} \mathrm{C} \mathrm{NMR}\left(101 \mathrm{MHz}, \mathrm{CDCl}_{3}\right) \delta 143.6$, 134.9, 133.6, 129.8, 127.7, 117.7, 70.6, 50.6, 50.5, 43.3, 42.6, 31.4, 21.7, 18.2, 15.4, 15.0, 12.4. Minor (distinguishable peaks): ${ }^{1} \mathrm{H}$ NMR (400 MHz, $\left.\mathrm{CDCl}_{3}\right) \delta 5.32(\mathrm{~s}, 1 \mathrm{H})$, 4.07-3.97 (m, 1H). HRMS (MALDI) calcd. for $\mathrm{C}_{26} \mathrm{H}_{43} \mathrm{NNaO}_{3} \mathrm{SSi}^{+}(\mathrm{M}+\mathrm{Na})^{+}: 500.2625$, Found: 500.2630 . The relative stereochemistry of this major compound was assigned by NOE experiment.<smiles>[3H]N1CC2=CC[C@H](OCC)[C@H](C(C)C)[C@@H]2C1</smiles>

( \pm )-4-Propyl-2-tosyl-5-((triethylsilyl)oxy)-2,3,3a,4,5,6-hexahydro-1H-isoindole (3r). Reaction conditions: $\mathrm{Et}_{3} \mathrm{SiH}$ (2.0 equiv), $\mathrm{B}\left(\mathrm{C}_{6} \mathrm{~F}_{5}\right)_{3}(5 \mathrm{~mol} \%), \mathrm{DCE}, 120{ }^{\circ} \mathrm{C}$. White solid (33 mg, 37\% yield). ${ }^{1} \mathrm{H}$ NMR (400 MHz, $\left.\mathrm{CDCl}_{3}\right) \delta 7.70(\mathrm{~d}, J=8.2 \mathrm{~Hz}, 2 \mathrm{H})$, $7.32(\mathrm{~d}, J=8.2 \mathrm{~Hz}, 2 \mathrm{H}), 5.39-5.34(\mathrm{~m}, 1 \mathrm{H}), 3.92(\mathrm{~d}, J=13.5 \mathrm{~Hz}, 1 \mathrm{H}), 3.85-3.78(\mathrm{~m}$, $1 \mathrm{H}), 3.60(\mathrm{t}, J=7.9 \mathrm{~Hz}, 1 \mathrm{H}), 3.55(\mathrm{~d}, J=13.5 \mathrm{~Hz}, 1 \mathrm{H}), 2.78(\mathrm{dd}, J=11.2,8.6 \mathrm{~Hz}, 1 \mathrm{H})$, 2.74-2.64 (m, 1H), $2.43(\mathrm{~s}, 3 \mathrm{H}), 2.12-2.01(\mathrm{~m}, 1 \mathrm{H}), 1.98-1.87(\mathrm{~m}, 1 \mathrm{H}), 1.78-1.71(\mathrm{~m}$, $1 \mathrm{H}), 1.47-1.34(\mathrm{~m}, 1 \mathrm{H}), 1.23-1.14(\mathrm{~m}, 2 \mathrm{H}), 0.90(\mathrm{t}, J=7.9 \mathrm{~Hz}, 9 \mathrm{H}), 0.82(\mathrm{t}, J=7.3 \mathrm{~Hz}$, 4H), 0.57-0.49 (m, 6H). $\left.{ }^{13} \mathrm{C} \mathrm{NMR} \mathrm{(101} \mathrm{MHz,} \mathrm{CDCl}_{3}\right) \delta 143.6,135.0,133.5,129.8$, 
127.8, 117.7, 70.1, 50.7, 50.5, 43.1, 40.3, 31.4, 25.1, 23.4, 21.7, 14.5, 7.0, 5.0. HRMS (MALDI) calcd. for $\mathrm{C}_{24} \mathrm{H}_{39} \mathrm{NNaO}_{3} \mathrm{SSi}^{+}(\mathrm{M}+\mathrm{Na})^{+}: 472.2312$, Found: 472.2318 .

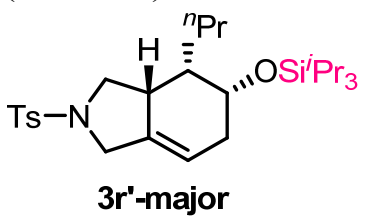

( \pm )-4-Propyl-2-tosyl-5-((triisopropylsilyl)oxy)-2,3,3a,4,5,6-hexahydro-1H-isoindol e (3r'). Reaction conditions: ${ }^{i} \operatorname{Pr}_{3} \mathrm{SiH}$ (1.5 equiv), $\mathrm{B}\left(\mathrm{C}_{6} \mathrm{~F}_{5}\right)_{3}(5 \mathrm{~mol} \%)$, toluene, $115^{\circ} \mathrm{C}$. White solid (43 mg, 44\% yield), $\mathrm{dr}=7.0: 1.0$. Major: ${ }^{1} \mathrm{H}$ NMR (400 MHz, $\left.\mathrm{CDCl}_{3}\right) \delta$ $7.70(\mathrm{~d}, J=8.1 \mathrm{~Hz}, 2 \mathrm{H}), 7.32(\mathrm{~d}, J=8.1 \mathrm{~Hz}, 2 \mathrm{H}), 5.40-5.34(\mathrm{~m}, 1 \mathrm{H}), 3.97-3.88(\mathrm{~m}, 2 \mathrm{H})$, $3.62(\mathrm{t}, J=7.6 \mathrm{~Hz}, 1 \mathrm{H}), 3.55(\mathrm{t}, J=13.7 \mathrm{~Hz}, 1 \mathrm{H}), 2.80-2.65(\mathrm{~m}, 2 \mathrm{H}), 2.43(\mathrm{~s}, 3 \mathrm{H})$, 2.18-2.09 (m, 1H), 1.97-1.88 (m, 1H), 1.86-1.79 (m, 1H), 1.51-1.42 (m, 1H), 1.23-1.15 $(\mathrm{m}, 2 \mathrm{H}), 1.05-0.94(\mathrm{~m}, 22 \mathrm{H}), 0.82(\mathrm{t}, J=7.3 \mathrm{~Hz}, 3 \mathrm{H}) .{ }^{13} \mathrm{C} \mathrm{NMR}\left(101 \mathrm{MHz}, \mathrm{CDCl}_{3}\right) \delta$ 143.6, 134.9, 133.5, 129.8, 127.8, 117.8, 70.5, 50.6, 50.6, 43.3, 40.6, 31.4, 23.7, 21.7, $18.2,18.2,14.7,12.4$. Minor (distinguishable peaks): ${ }^{1} \mathrm{H} \mathrm{NMR}\left(400 \mathrm{MHz}, \mathrm{CDCl}_{3}\right) \delta$ $5.32(\mathrm{~s}, 1 \mathrm{H}), 4.01-4.98(\mathrm{~m}, 1 \mathrm{H}), 2.94-2.86(\mathrm{~m}, 2 \mathrm{H})$. HRMS (MALDI) calcd. for $\mathrm{C}_{27} \mathrm{H}_{45} \mathrm{NNaO}_{3} \mathrm{SSi}^{+}(\mathrm{M}+\mathrm{Na})^{+}:$514.2782, Found: 514.2786.

\section{Transformations of Product 4a}

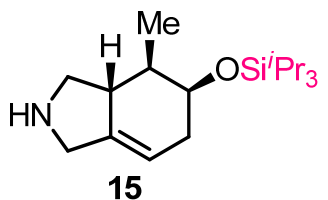

( \pm )-4-Methyl-5-((triisopropylsilyl)oxy)-2,3,3a,4,5,6-hexahydro-1H-isoindole (15). Magnesium turnings ( $84 \mathrm{mg}, 3.84 \mathrm{mmol}, 20$ equiv) were added to a solution of $\mathbf{4 a}$ (80 $\mathrm{mg}, 0.19 \mathrm{mmol}, 1$ equiv) in dry $\mathrm{MeOH}(4 \mathrm{~mL})$, and the mixture was refluxed for 1 hours under argon. After being allowed to cool to room temperature, the suspension was filtered through a short plug of Celite. The filtrate was concentrated in vacuo, and the residue was purified by flash column chromatography (from $\mathrm{MeOH} / \mathrm{DCM}=10: 1$ to $\left.\mathrm{MeOH} / \mathrm{DCM} / \mathrm{NH}_{4} \mathrm{OH}=8: 1: 0.08\right)$ to afford the title compound as a colorless oil (41 mg, $70 \%$ yield). ${ }^{1} \mathrm{H}$ NMR $\left(400 \mathrm{MHz}, \mathrm{CDCl}_{3}\right) \delta 5.35(\mathrm{~s}, 1 \mathrm{H}), 4.07-3.93(\mathrm{~m}, 1 \mathrm{H}), 3.69(\mathrm{~d}$, $J=14.4 \mathrm{~Hz}, 1 \mathrm{H}), 3.55-3.42(\mathrm{~m}, 2 \mathrm{H}), 3.40-3.31(\mathrm{~m}, 1 \mathrm{H}), 2.46-2.33(\mathrm{~m}, 2 \mathrm{H}), 2.33-2.14$ (m, 2H), 1.33-1.25 (m, 1H), 1.08-0.98 (m, 24H). $\left.{ }^{13} \mathrm{C} \mathrm{NMR} \mathrm{(101} \mathrm{MHz,} \mathrm{CDCl}_{3}\right) \delta 141.0$, 114.1, 70.9, 52.2, 49.9, 41.8, 39.1, 35.8, 18.4, 18.3, 17.5, 13.1. HRMS (ESI) calcd. for $\mathrm{C}_{15} \mathrm{H}_{36} \mathrm{NOSi}^{+}(\mathrm{M}+\mathrm{H})^{+}: 310.2561$, Found: 310.2565 .

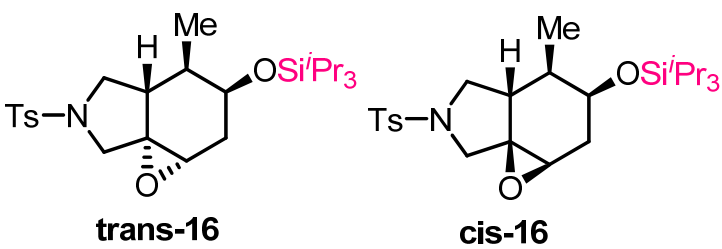

( \pm )-4-Methyl-6-tosyl-3-((triisopropylsilyl)oxy)octahydrooxireno[2,3-d]isoindole (16). $m$-CPBA ( $34.5 \mathrm{mg}, 0.2 \mathrm{mmol}, 2$ equiv) was added in portions to a solution of $4 \mathbf{a}$ (46.4 mg, $0.1 \mathrm{mmol}, 1$ equiv) in dry DCM $(8 \mathrm{~mL})$ at $0{ }^{\circ} \mathrm{C}$. The reaction mixture was 
allowed to warm to room temperature and stirred for $16 \mathrm{~h}$. The mixture was washed with aqueous $\mathrm{Na}_{2} \mathrm{~S}_{2} \mathrm{O}_{3}$. The organic layer was dried, filtered and concentrated in vacuo. The residue was purified by column chromatography $(\mathrm{PE} / \mathrm{EA}=10: 1)$ to afford ( \pm ) trans-16 (32 mg, 67\% yield) and ( \pm ) cis-16 (12 mg, 25\% yield).

( \pm ) trans-16: White solid. ${ }^{1} \mathrm{H}$ NMR $\left(400 \mathrm{MHz}, \mathrm{CDCl}_{3}\right) \delta 7.71(\mathrm{~d}, J=8.2 \mathrm{~Hz}, 2 \mathrm{H}), 7.31$ $(\mathrm{d}, J=8.2 \mathrm{~Hz}, 2 \mathrm{H}), 3.82-3.71(\mathrm{~m}, 3 \mathrm{H}), 3.40(\mathrm{~d}, J=3.5 \mathrm{~Hz}, 1 \mathrm{H}), 3.14(\mathrm{~d}, J=11.4 \mathrm{~Hz}$, $1 \mathrm{H}), 2.90(\mathrm{dd}, J=12.3,8.8 \mathrm{~Hz}, 1 \mathrm{H}), 2.41(\mathrm{~s}, 3 \mathrm{H}), 2.35(\mathrm{td}, J=11.8,7.3 \mathrm{~Hz}, 1 \mathrm{H})$, 2.06-1.91 (m, 2H), $1.55(\mathrm{dq}, J=13.7,6.9 \mathrm{~Hz}, 1 \mathrm{H}), 1.02-0.95(\mathrm{~m}, 21 \mathrm{H}), 0.90(\mathrm{~d}, J=6.9$ $\mathrm{Hz}, 3 \mathrm{H}) .{ }^{13} \mathrm{C} \mathrm{NMR}\left(101 \mathrm{MHz}, \mathrm{CDCl}_{3}\right) \delta 143.6,134.3,129.9,127.5,70.1,67.1,53.4$, 52.4, 50.2, 39.1, 34.5, 32.7, 21.7, 18.3, 18.2, 16.3, 12.9. HRMS (MALDI) calcd. for $\mathrm{C}_{25} \mathrm{H}_{41} \mathrm{NNaO}_{4} \mathrm{SSi}^{+} \quad(\mathrm{M}+\mathrm{Na})^{+}:$502.2418, Found: 502.2423. The relative stereochemistry of this compound was assigned by NOE experiment.

( \pm ) cis-16: White solid. ${ }^{1} \mathrm{H}$ NMR $\left(400 \mathrm{MHz}, \mathrm{CDCl}_{3}\right) \delta 7.71(\mathrm{~d}, J=8.1 \mathrm{~Hz}, 2 \mathrm{H}), 7.35$ (d, $J=8.1 \mathrm{~Hz}, 2 \mathrm{H}), 3.89-3.77(\mathrm{~m}, 1 \mathrm{H}), 3.61(\mathrm{t}, J=9.5 \mathrm{~Hz}, 1 \mathrm{H}), 3.33-3.25(\mathrm{~m}, 2 \mathrm{H})$, 3.14-3.09 (m, 1H), $2.94(\mathrm{dd}, J=10.0,8.0 \mathrm{~Hz}, 1 \mathrm{H}), 2.44(\mathrm{~s}, 3 \mathrm{H}), 2.34-2.19(\mathrm{~m}, 2 \mathrm{H})$, 1.87-1.77 (m, 1H), 1.29-1.24 (m, 1H), 1.06-0.91 (m, 24H). $\left.{ }^{13} \mathrm{C} \mathrm{NMR} \mathrm{(101} \mathrm{MHz,} \mathrm{CDCl}_{3}\right)$ $\delta 144.1,132.1,129.9,128.2,69.3,63.8,56.1,52.4,52.0,39.0,37.9,33.1,21.7,18.3$, 18.2, 17.0, 12.8. HRMS (MALDI) calcd. for $\mathrm{C}_{25} \mathrm{H}_{41} \mathrm{NNaO}_{4} \mathrm{SSi}^{+}(\mathrm{M}+\mathrm{Na})^{+}$: 502.2418, Found: 502.2422 .

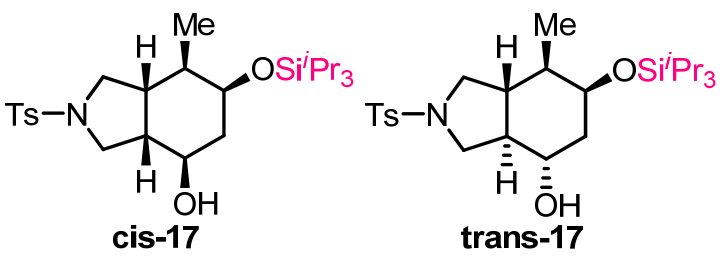

( \pm )-7-Methyl-2-tosyl-6-((triisopropylsilyl)oxy)octahydro-1H-isoindol-4-ol (17). 4a (120 mg, $0.26 \mathrm{mmol}, 1$ equiv) was dissolved in dry THF $(8 \mathrm{~mL})$. The solution was cooled to $0{ }^{\circ} \mathrm{C}$, and then $\mathrm{BH}_{3} \cdot \mathrm{THF}(1.29 \mathrm{~mL}, 1.29 \mathrm{mmol}, 5$ equiv) was added dropwise. The mixture was stirred overnight at room temperature under argon. The mixture was then cooled to $0{ }^{\circ} \mathrm{C}$, and treated dropwise with aqueous $\mathrm{NaOH}(10 \%, 2$ $\mathrm{mL})$ and subsequently aqueous $\mathrm{H}_{2} \mathrm{O}_{2}(30 \%, 2 \mathrm{~mL})$. This mixture was heated to $60{ }^{\circ} \mathrm{C}$ for $1 \mathrm{~h}$. The volatiles were removed in vacuo, followed by the addition of $\mathrm{H}_{2} \mathrm{O}(30$ $\mathrm{mL})$. The mixture was extracted with ethyl acetate $(3 \times 5 \mathrm{~mL})$. The combined organic layers were dried, filtered, and concentrated to give a viscous liquid which was chromatographed $(\mathrm{PE} / \mathrm{EA}=5: 1)$ on silica gel to afford $( \pm)$ cis-17 (43.9 mg, 35\% yield) and $( \pm)$ trans-17 (43.8 $\mathrm{mg}, 35 \%$ yield).

( \pm ) cis-17: Colorless oil. ${ }^{1} \mathrm{H}$ NMR $\left(400 \mathrm{MHz}, \mathrm{CDCl}_{3}\right) \delta 7.71(\mathrm{~d}, J=8.1 \mathrm{~Hz}, 2 \mathrm{H}), 7.32$ $(\mathrm{d}, J=8.1 \mathrm{~Hz}, 2 \mathrm{H}), 4.01-3.94(\mathrm{~m}, 1 \mathrm{H}), 3.49-3.41(\mathrm{~m}, 1 \mathrm{H}), 3.31-3.19(\mathrm{~m}, 4 \mathrm{H}$,$) ,$ 3.16-3.04 (m, 1H), $2.42(\mathrm{~s}, 3 \mathrm{H}), 2.23-2.05(\mathrm{~m}, 2 \mathrm{H}), 1.92-1.79(\mathrm{~m}, 1 \mathrm{H}), 1.66(\mathrm{dt}, J=$ 13.7, $3.3 \mathrm{~Hz}, 1 \mathrm{H}), 1.57-1.46(\mathrm{~m}, 1 \mathrm{H}), 1.11-0.99(\mathrm{~m}, 21 \mathrm{H}), 0.96(\mathrm{~d}, J=6.9 \mathrm{~Hz}, 3 \mathrm{H})$. ${ }^{13} \mathrm{C}$ NMR $\left(101 \mathrm{MHz}, \mathrm{CDCl}_{3}\right) \delta 143.6,133.9,129.8,127.5,70.8,67.1,51.0,49.5,44.4$, 40.2, 35.6, 34.4, 21.7, 18.3, 18.2, 15.5, 12.6. HRMS (ESI) calcd. for $\mathrm{C}_{25} \mathrm{H}_{43} \mathrm{NNaO}_{4} \mathrm{SSi}^{+} \quad(\mathrm{M}+\mathrm{Na})^{+}:$504.2574, Found: 504.2578. The relative stereochemistry of this compound was assigned by NOE experiment. 
( \pm ) trans-17: White solid. ${ }^{1} \mathrm{H}$ NMR $\left(400 \mathrm{MHz}, \mathrm{CDCl}_{3}\right) \delta 7.70(\mathrm{~d}, J=8.1 \mathrm{~Hz}, 2 \mathrm{H}), 7.30$ $(\mathrm{d}, J=8.1 \mathrm{~Hz}, 2 \mathrm{H}), 4.07-4.03(\mathrm{~m}, 1 \mathrm{H}), 3.79-3.66(\mathrm{~m}, 2 \mathrm{H}), 3.58(\mathrm{dd}, J=9.1,7.1 \mathrm{~Hz}, 1 \mathrm{H})$, $3.03(\mathrm{t}, J=10.2 \mathrm{~Hz}, 1 \mathrm{H}), 2.79(\mathrm{dd}, J=11.4,9.1 \mathrm{~Hz}, 1 \mathrm{H}), 2.41$ (s, 3H), 2.11 (dt, $J=13.2$, $3.8 \mathrm{~Hz}, 1 \mathrm{H}), 1.79(\mathrm{~s}, 1 \mathrm{H}), 1.77-1.63(\mathrm{~m}, 1 \mathrm{H}), 1.47-1.37(\mathrm{~m}, 1 \mathrm{H}), 1.37-1.25(\mathrm{~m}, 2 \mathrm{H})$, 1.07-0.94 (m, 21H), $0.89(\mathrm{~d}, J=6.8 \mathrm{~Hz}, 3 \mathrm{H}) .{ }^{13} \mathrm{C} \mathrm{NMR}\left(101 \mathrm{MHz}, \mathrm{CDCl}_{3}\right) \delta 143.4$, 134.6, 129.8, 127.4, 73.3, 69.3, 52.2, 52.1, 51.6, 43.8, 42.0, 40.3, 21.7, 18.3, 18.3, 16.2, 12.9. HRMS (ESI) calcd. for $\mathrm{C}_{25} \mathrm{H}_{43} \mathrm{NNaO}_{4} \mathrm{SSi}^{+}(\mathrm{M}+\mathrm{Na})^{+}: 504.2574$, Found: 504.2578.<smiles>[Y5]N1CC2=CC[C@@H](O)[C@H]([C@@H]2C)[C@H]1C</smiles>

18

( \pm )-4-Methyl-2-tosyl-2,3,3a,4,5,6-hexahydro-1H-isoindol-5-ol (18). 4a (50 mg, 0.11 mmol, 1 equiv) was dissolved in dry THF $(5 \mathrm{~mL})$. The solution was cooled to $0{ }^{\circ} \mathrm{C}$, and then TBAF in THF $(0.22 \mathrm{~mL}, 1 \mathrm{M}, 0.216 \mathrm{mmol}, 2$ equiv) was added dropwise. The resulting mixture was stirred overnight at room temperature. After the addition of $\mathrm{H}_{2} \mathrm{O}(5 \mathrm{~mL})$, the mixture was extracted with ethyl acetate $(3 \times 5 \mathrm{~mL})$. The combined organic layers were dried, filtered, and concentrated in vacuo. The residue was purified by column chromatography $(\mathrm{PE} / \mathrm{EA}=3: 2)$ to afford the title compound as a colorless oil $(28 \mathrm{mg}, 84 \%) .{ }^{1} \mathrm{H}$ NMR $\left(400 \mathrm{MHz}, \mathrm{CDCl}_{3}\right) \delta 7.70(\mathrm{~d}, J=8.1 \mathrm{~Hz}, 2 \mathrm{H})$, $7.31(\mathrm{~d}, J=8.1 \mathrm{~Hz}, 2 \mathrm{H}), 5.38(\mathrm{~s}, 1 \mathrm{H}), 3.99(\mathrm{~d}, J=13.3 \mathrm{~Hz}, 1 \mathrm{H}), 3.85-3.78(\mathrm{~m}, 2 \mathrm{H}), 3.70$ $(\mathrm{d}, J=13.3 \mathrm{~Hz}, 1 \mathrm{H}), 2.63(\mathrm{dd}, J=10.6,9.1 \mathrm{~Hz}, 1 \mathrm{H}), 2.48-2.38(\mathrm{~m}, 4 \mathrm{H}), 2.37-2.27(\mathrm{~m}$, 1H), $2.16(\mathrm{~d}, J=18.8 \mathrm{~Hz}, 1 \mathrm{H}), 1.69(\mathrm{br}, 1 \mathrm{H}), 1.34-1.26(\mathrm{~m}, 1 \mathrm{H}), 1.01(\mathrm{~d}, J=6.8 \mathrm{~Hz}$, $3 \mathrm{H}) .{ }^{13} \mathrm{C} \mathrm{NMR}\left(101 \mathrm{MHz}, \mathrm{CDCl}_{3}\right) \delta 143.6,136.2,133.8,129.8,127.6,116.0,69.1,53.3$, 51.4, 39.8, 37.2, 34.7, 21.6, 16.7. HRMS (ESI) calcd. for $\mathrm{C}_{16} \mathrm{H}_{21} \mathrm{NNaO}_{3} \mathrm{~S}^{+}(\mathrm{M}+\mathrm{Na})^{+}$: 330.1134, Found: 330.1138.<smiles>[3H]N1CC2=CCC(=O)[CH][C@@H]2C1</smiles>

12

( \pm )-4-Methyl-2-tosyl-1,2,3,3a,4,6-hexahydro-5H-isoindol-5-one. (12). PCC (86.2 $\mathrm{mg}, 0.4 \mathrm{mmol}, 4$ equiv) was added in portions to a solution of $18(30.7 \mathrm{mg}, 0.1 \mathrm{mmol}$, 1 equiv) in dry DCM $(5 \mathrm{~mL})$. The resulting mixture was stirred at room temperature for $16 \mathrm{~h}$. The reaction mixture was then filtered through a plug of Celite. The filtrate was concentrated in vacuo, and the residue was purified by column chromatography $(\mathrm{PE} / \mathrm{EA}=4: 1)$ to afford the title compound as a white solid (30 mg, 98\% yield). ${ }^{1} \mathrm{H}$ NMR (400 MHz, $\left.\mathrm{CDCl}_{3}\right) \delta 7.72(\mathrm{~d}, J=8.1 \mathrm{~Hz}, 2 \mathrm{H}), 7.34(\mathrm{~d}, J=8.1 \mathrm{~Hz}, 2 \mathrm{H}), 5.65-5.59$ $(\mathrm{m}, 1 \mathrm{H}), 4.06(\mathrm{~d}, J=14.0 \mathrm{~Hz}, 1 \mathrm{H}), 3.89(\mathrm{t}, J=8.6 \mathrm{~Hz}, 1 \mathrm{H}), 3.77(\mathrm{~d}, J=14.0 \mathrm{~Hz}, 1 \mathrm{H})$, 2.96-2.84 (m, 1H), 2.84-2.72 (m, 2H), 2.65-2.43 (m, 1H), $2.45(\mathrm{~s}, 3 \mathrm{H}), 2.23-2.14(\mathrm{~m}$, $1 \mathrm{H}), 1.05(\mathrm{~d}, J=6.5 \mathrm{~Hz}, 3 \mathrm{H}) .{ }^{13} \mathrm{C} \mathrm{NMR}\left(101 \mathrm{MHz}, \mathrm{CDCl}_{3}\right) \delta 208.9,144.0,137.9,133.4$, 130.0, 127.8, 116.5, 54.1, 51.0, 46.6, 45.9, 39.0, 21.7, 12.1. HRMS (ESI) calcd. for $\mathrm{C}_{16} \mathrm{H}_{19} \mathrm{NNaO}_{3} \mathrm{~S}^{+}(\mathrm{M}+\mathrm{Na})^{+}$: 328.0978, Found: 328.0984 . 


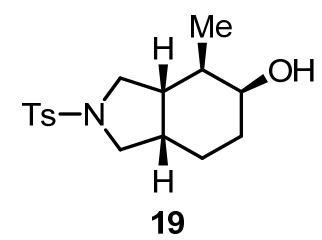

( \pm )-4-Methyl-2-tosyloctahydro-1H-isoindol-5-ol (19). 18 (38 mg, $0.12 \mathrm{mmol}, 1$ equiv) and $\mathrm{Pd} / \mathrm{C}(3.8 \mathrm{mg}, 10 \%)$ were dissolved in $\mathrm{DCM}(3 \mathrm{~mL})$ in a $10 \mathrm{~mL}$ glass test tube. The tube was then placed in a stainless-steel autoclave. After being sealed, the autoclave was purged three times with $\mathrm{H}_{2}$ and the final pressure of hydrogen was adjusted to 5 bar. After being stirred at room temperature for $4 \mathrm{~h}$, the reaction mixture was filtered through a short plug of Celite. The filtrate was then concentrated, and purified by column chromatography $(\mathrm{PE} / \mathrm{EA}=1.5: 1)$ to afford the title compound as a colorless oil (37 mg, 99\% yield), $\mathrm{dr}=13.0: 1.0$. Major $\left(( \pm)\right.$ cis-19): ${ }^{1} \mathrm{H}$ NMR (400 MHz, $\left.\mathrm{CDCl}_{3}\right) \delta 7.70(\mathrm{~d}, J=8.1 \mathrm{~Hz}, 2 \mathrm{H}), 7.30(\mathrm{~d}, J=8.1 \mathrm{~Hz}, 2 \mathrm{H}), 3.74-3.68(\mathrm{~m}, 1 \mathrm{H}), 3.32(\mathrm{dd}$, $J=10.0,2.7 \mathrm{~Hz}, 1 \mathrm{H}), 3.26-3.16(\mathrm{~m}, 2 \mathrm{H}), 3.12(\mathrm{t}, J=9.5 \mathrm{~Hz}, 1 \mathrm{H}), 2.42(\mathrm{~s}, 3 \mathrm{H})$, 2.21-2.12 (m, 1H), 1.98-1.91 (m, 1H), 1.96-1.74 (m, 1H), 1.62-1.54 (m, 1H), 1.52-1.41 $(\mathrm{m}, 2 \mathrm{H}), 1.33-1.24(\mathrm{~m}, 1 \mathrm{H}), 1.24-1.17(\mathrm{~m}, 1 \mathrm{H}), 0.88(\mathrm{~d}, J=6.8 \mathrm{~Hz}, 3 \mathrm{H}) .{ }^{13} \mathrm{C}$ NMR $(101$ $\left.\mathrm{MHz}, \mathrm{CDCl}_{3}\right) \delta 143.4,134.2,129.7,127.4,69.6,51.7,49.6,41.0,36.1,33.6,27.7,21.6$, 19.3, 16.1. Minor (( \pm ) trans-19, distinguishable peaks): ${ }^{1} \mathrm{H}$ NMR $\left(400 \mathrm{MHz}, \mathrm{CDCl}_{3}\right) \delta$ $3.76(\mathrm{~s}, 1 \mathrm{H}), 3.53-3.46(\mathrm{~m}, 2 \mathrm{H}), 2.83-2.74(\mathrm{~m}, 2 \mathrm{H}), 0.97$ (d, J=7.3 Hz, 3H). HRMS (ESI) calcd. for $\mathrm{C}_{16} \mathrm{H}_{22} \mathrm{NO}_{3} \mathrm{~S}^{+}(\mathrm{M}+\mathrm{H})^{+}: 310.1471$, Found: 310.1478 . The relative stereochemistry of this major compound was assigned by NOE experiment.

\section{Mechanistic Studies}

\subsection{Deuterium-Labelling Experiments}

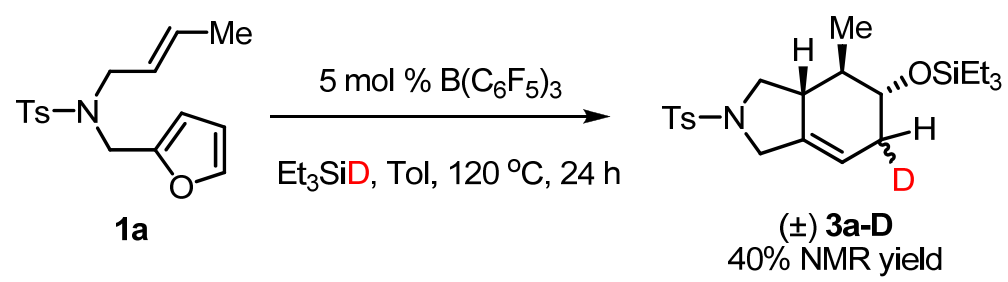

In a glovebox, Et ${ }_{3} \mathrm{SiD}$ ( $48 \mu \mathrm{L}, 0.3 \mathrm{mmol}, 1.5$ equiv) was added to a solution of $1 \mathrm{a}(61$ $\mathrm{mg}, 0.2 \mathrm{mmol}, 1$ equiv) in dry toluene $(0.5 \mathrm{~mL})$ in an $8 \mathrm{~mL}$ vial. $\mathrm{B}\left(\mathrm{C}_{6} \mathrm{~F}_{5}\right)_{3}(5.1 \mathrm{mg}$, $0.01 \mathrm{mmol}, 5 \mathrm{~mol} \%$ ) was then added before the vial was capped and closed tightly. The reaction mixture was stirred at $120{ }^{\circ} \mathrm{C}$ for $24 \mathrm{~h}$. After being allowed to cool to room temperature, one drop Et $3 \mathrm{~N}$ was added to the solution. After evaporation of the solvent in vacuo, the residue was subjected to column chromatography on silica gel ( $\mathrm{PE} / \mathrm{EA}=30: 1$ to $20: 1$ as eluent) to give the product. 
<smiles>[2H][C@H]1C=C2CN([As])C[C@H]2[C@H](OCC)[C@H]1OC</smiles>

3a-D-major

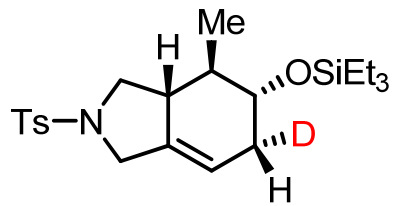

3a-D-minor

3a-D: $\mathrm{dr}=7: 3$. White solid. ${ }^{1} \mathrm{H}$ NMR $\left(400 \mathrm{MHz}, \mathrm{CDCl}_{3}\right.$ ) (mixture of two diastereomers) $\delta 7.69(\mathrm{~d}, J=7.9 \mathrm{~Hz}, 2 \mathrm{H}), 7.32(\mathrm{~d}, J=7.9 \mathrm{~Hz}, 2 \mathrm{H}), 5.37(\mathrm{~s}, 1 \mathrm{H})$, 3.99-3.91 (m, 1H), 3.82-3.76 (m, 1H), 3.67-3.59 (m, 1H), 3.52-3.44 (m, 1H), $2.59(\mathrm{t}, J$ $=9.8 \mathrm{~Hz}, 1 \mathrm{H}), 2.43(\mathrm{~s}, 3 \mathrm{H}), 2.32-2.18(\mathrm{~m}, 1.3 \mathrm{H}), 2.00-1.90(\mathrm{~m}, 0.7 \mathrm{H}), 1.30-1.22(\mathrm{~m}$, $1 \mathrm{H}), 0.99-0.87(\mathrm{~m}, 12 \mathrm{H}), 0.63-0.52(\mathrm{~m}, 6 \mathrm{H})$.

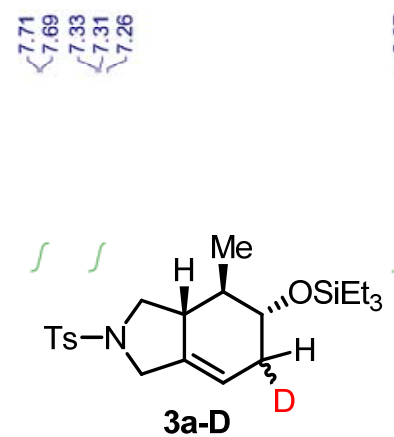

ले

.

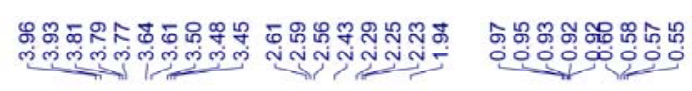

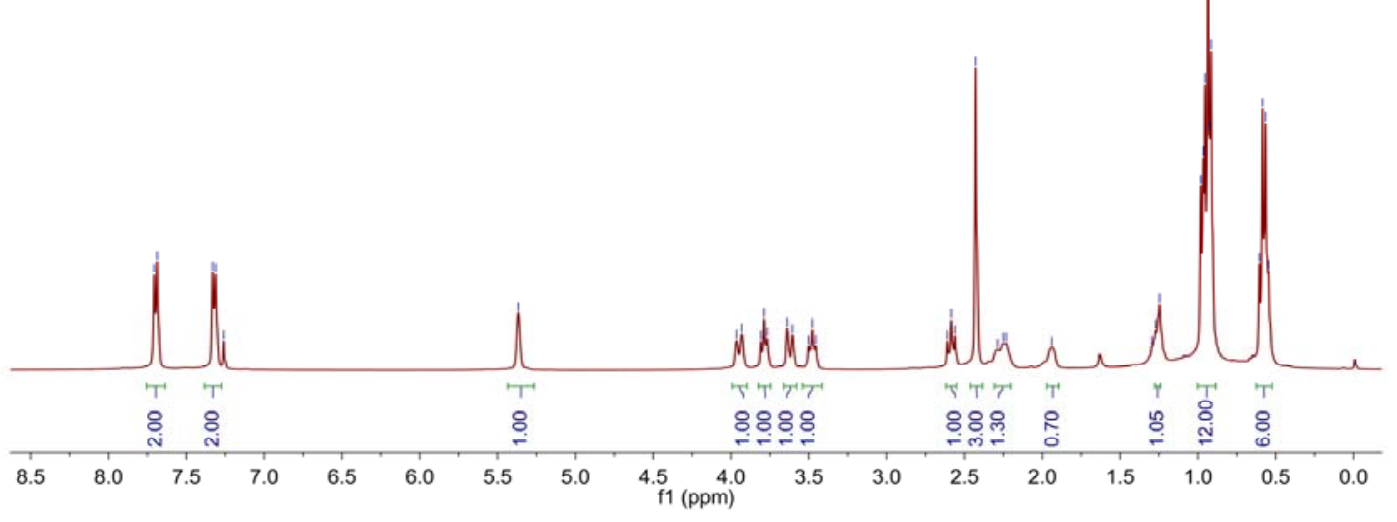

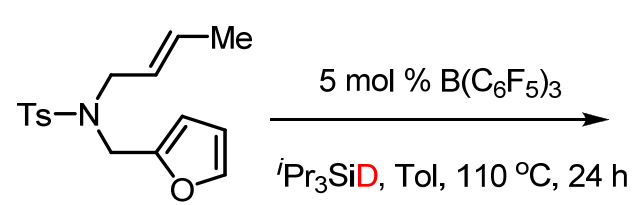

$1 a$

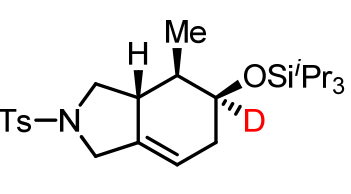

( \pm ) 4a-D

$86 \%$ NMR yield

In a glovebox, ${ }^{i} \mathrm{Pr}_{3} \mathrm{SiD}(61 \mu \mathrm{L}, 0.3 \mathrm{mmol}, 1.5$ equiv) was added to a solution of $1 \mathbf{a}$ (61 $\mathrm{mg}, 0.2 \mathrm{mmol}, 1$ equiv) in dry toluene $(0.5 \mathrm{~mL})$ in an $8 \mathrm{~mL}$ vial. $\mathrm{B}\left(\mathrm{C}_{6} \mathrm{~F}_{5}\right)_{3}(5.1 \mathrm{mg}$, $0.01 \mathrm{mmol}, 5 \mathrm{~mol} \%$ ) was then added before the vial was capped and closed tightly. The reaction mixture was stirred at $110{ }^{\circ} \mathrm{C}$ for $24 \mathrm{~h}$. After being allowed to cool to room temperature, one drop of $\mathrm{Et}_{3} \mathrm{~N}$ was added to the solution. After evaporation of the solvent in vacuo, the residue was subjected to column chromatography on silica gel (PE/EA $=30: 1$ to 20:1 as eluent) to give the product. 


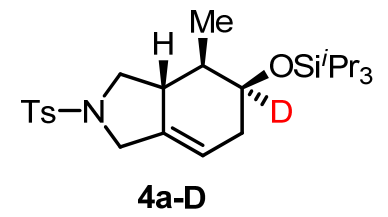

4a-D: White solid. ${ }^{1} \mathrm{H}$ NMR $\left(400 \mathrm{MHz}, \mathrm{CDCl}_{3}\right) \delta 7.72(\mathrm{~d}, J=8.0 \mathrm{~Hz}, 2 \mathrm{H}), 7.31(\mathrm{~d}, J$ $=8.0 \mathrm{~Hz}, 2 \mathrm{H}), 5.31(\mathrm{~s}, 1 \mathrm{H}), 4.09-3.95(\mathrm{~m}, 1 \mathrm{H}), 3.86-3.79(\mathrm{~m}, 1 \mathrm{H}), 3.67(\mathrm{~d}, J=13.5$ $\mathrm{Hz}, 1 \mathrm{H}), 2.66-2.54(\mathrm{~m}, 1 \mathrm{H}), 2.55-2.38(\mathrm{~m}, 4 \mathrm{H}), 2.27-2.07(\mathrm{~m}, 2 \mathrm{H}), 1.27-1.17(\mathrm{~m}, 1 \mathrm{H})$, $1.12-0.84(\mathrm{~m}, 24 \mathrm{H})$.

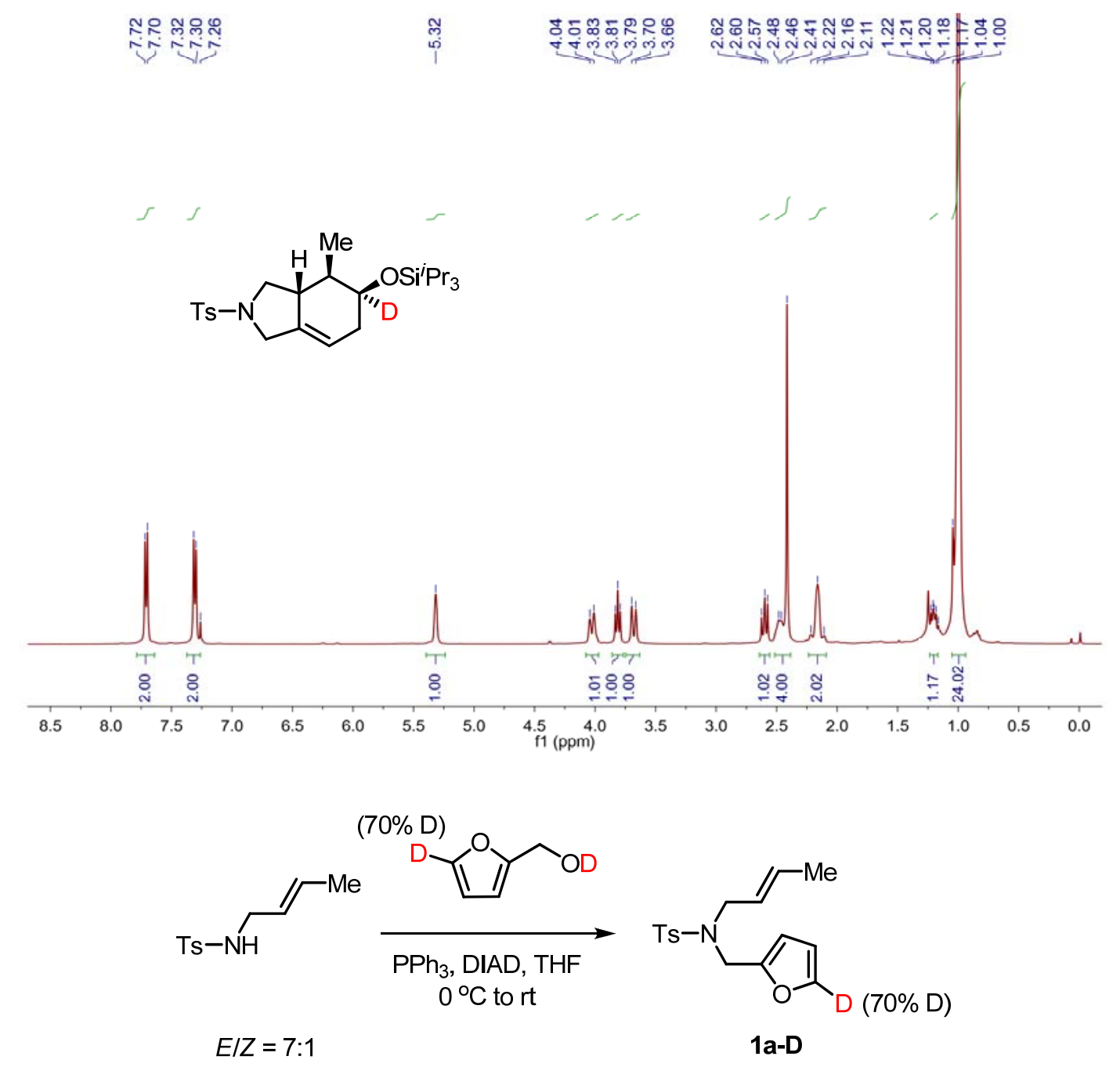

The deuterated furfuryl alcohol ${ }^{[11]}\left(0.72 \mathrm{~g}, 7.2 \mathrm{mmol}, 1.2\right.$ equiv) and $\mathrm{PPh}_{3}(2.20 \mathrm{~g}, 8.4$ mmol, 1.4 equiv) were added to a solution of the tosylated but-2-en-1-amine ${ }^{[12]}(1.35 \mathrm{~g}$, $6 \mathrm{mmol}, 1$ equiv) in dry THF $(20 \mathrm{~mL})$ under argon. Diisopropyl azodicarboxylate ( $1.70 \mathrm{~g}, 8.4 \mathrm{mmol}, 1.4$ equiv) was then added dropwise at $0{ }^{\circ} \mathrm{C}$, and the resulting mixture was stirred for $5 \mathrm{~h}$ at room temperature. The mixture was concentrated in vacuo. The residue was then subjected to column chromatography on silica gel $(\mathrm{PE} / \mathrm{EA}=20: 1$ as eluent) to give the product. 


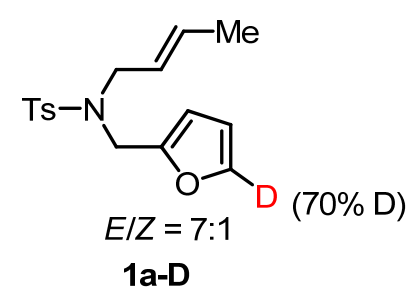

1a-D: Colorless oil, $E / Z=7: 1 .{ }^{1} \mathrm{H}$ NMR $\left(400 \mathrm{MHz}, \mathrm{CDCl}_{3}\right) \delta(E): 7.62(\mathrm{~d}, J=8.3 \mathrm{~Hz}$, $2 \mathrm{H})$, 7.25-7.20 (m, 2.3H), 6.26-6.20 (m, 1H), 6.19-6.01 (m, 1H), 5.62-5.53 (m, 1H), $5.35-5.19(\mathrm{~m}, 1 \mathrm{H}), 4.38(\mathrm{~s}, 2 \mathrm{H}), 3.71(\mathrm{~d}, J=6.6 \mathrm{~Hz}, 1.75 \mathrm{H}), 2.41(\mathrm{~s}, 3 \mathrm{H}), 1.65(\mathrm{~d}, J=$ 6.5, 2.63H); (Z) (distinguishable peaks): $3.84(\mathrm{~d}, J=7.0 \mathrm{~Hz}, 0.25 \mathrm{H}), 1.56(\mathrm{~d}, J=6.9$, $0.38 \mathrm{H})$.
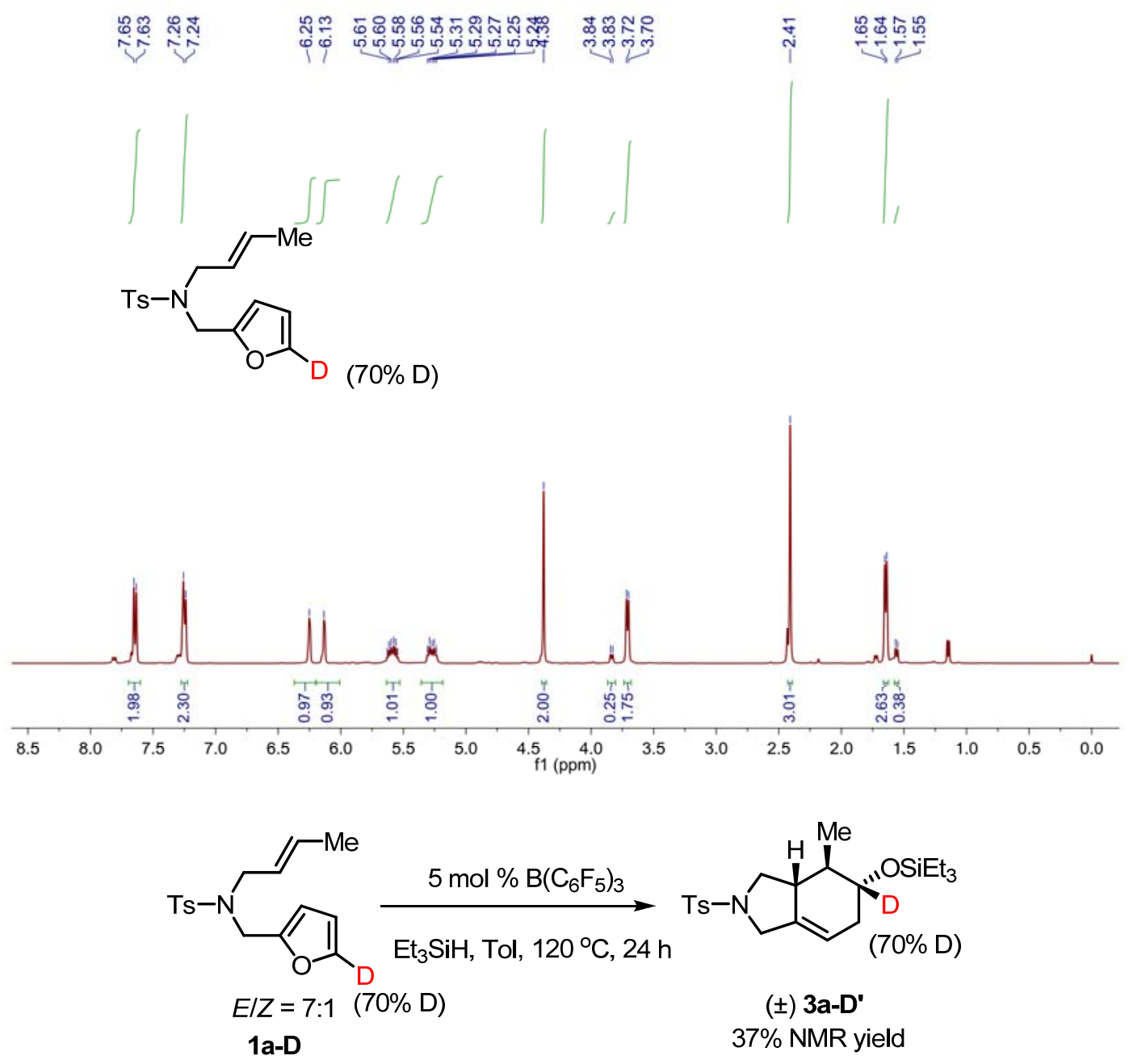

In a glovebox, $\mathrm{Et}_{3} \mathrm{SiH}$ ( $48 \mathrm{uL}, 0.3 \mathrm{mmol}, 1.5$ equiv) was added to a solution of 1a-D (61 mg, $0.2 \mathrm{mmol}, 1$ equiv) in dry toluene $(0.5 \mathrm{~mL})$ in an $8 \mathrm{~mL}$ vial. $\mathrm{B}\left(\mathrm{C}_{6} \mathrm{~F}_{5}\right)_{3}(5.1 \mathrm{mg}$, $0.01 \mathrm{mmol}, 5 \mathrm{~mol} \%$ ) was then added before the vial was capped and closed tightly. The reaction mixture was stirred at $120{ }^{\circ} \mathrm{C}$ for $24 \mathrm{~h}$. After being allowed to cool to room temperature, one drop of $\mathrm{Et}_{3} \mathrm{~N}$ was added to the solution. After evaporation of the solvent in vacuo, the residue was subjected to column chromatography on silica gel $(\mathrm{PE} / \mathrm{EA}=30: 1$ to $20: 1$ as eluent $)$ to give the product. We did not observe the product from the reaction of the $Z$ isomer of $\mathbf{1 a - D .}$ 
<smiles>[M]C1C2CN([3H])CC2C=C[C@@]1(O)OCC</smiles>

3a-D': White solid. ${ }^{1} \mathrm{H}$ NMR (400 MHz, $\left.\mathrm{CDCl}_{3}\right) \delta 7.69(\mathrm{~d}, J=7.9 \mathrm{~Hz}, 2 \mathrm{H}), 7.32(\mathrm{~d}, J=$ $7.9 \mathrm{~Hz}, 2 \mathrm{H}), 5.37(\mathrm{~s}, 1 \mathrm{H}), 3.95(\mathrm{~d}, J=13.5 \mathrm{~Hz}, 1 \mathrm{H}), 3.79(\mathrm{t}, J=8.4 \mathrm{~Hz}, 1 \mathrm{H}), 3.63(\mathrm{~d}, J$ $=13.5 \mathrm{~Hz}, 1 \mathrm{H}), 3.52-3.44(\mathrm{~m}, 0.3 \mathrm{H}), 2.59(\mathrm{t}, J=9.8 \mathrm{~Hz}, 1 \mathrm{H}), 2.43(\mathrm{~s}, 3 \mathrm{H}), 2.38-2.18(\mathrm{~m}$, $2 \mathrm{H}), 1.96(\mathrm{~d}, J=17.6 \mathrm{~Hz}, 1 \mathrm{H}), 1.30-1.22(\mathrm{~m}, 1 \mathrm{H}), 0.99-0.90(\mathrm{~m}, 12 \mathrm{H}), 0.58(\mathrm{q}, J=7.9$ $\mathrm{Hz}, 6 \mathrm{H})$.
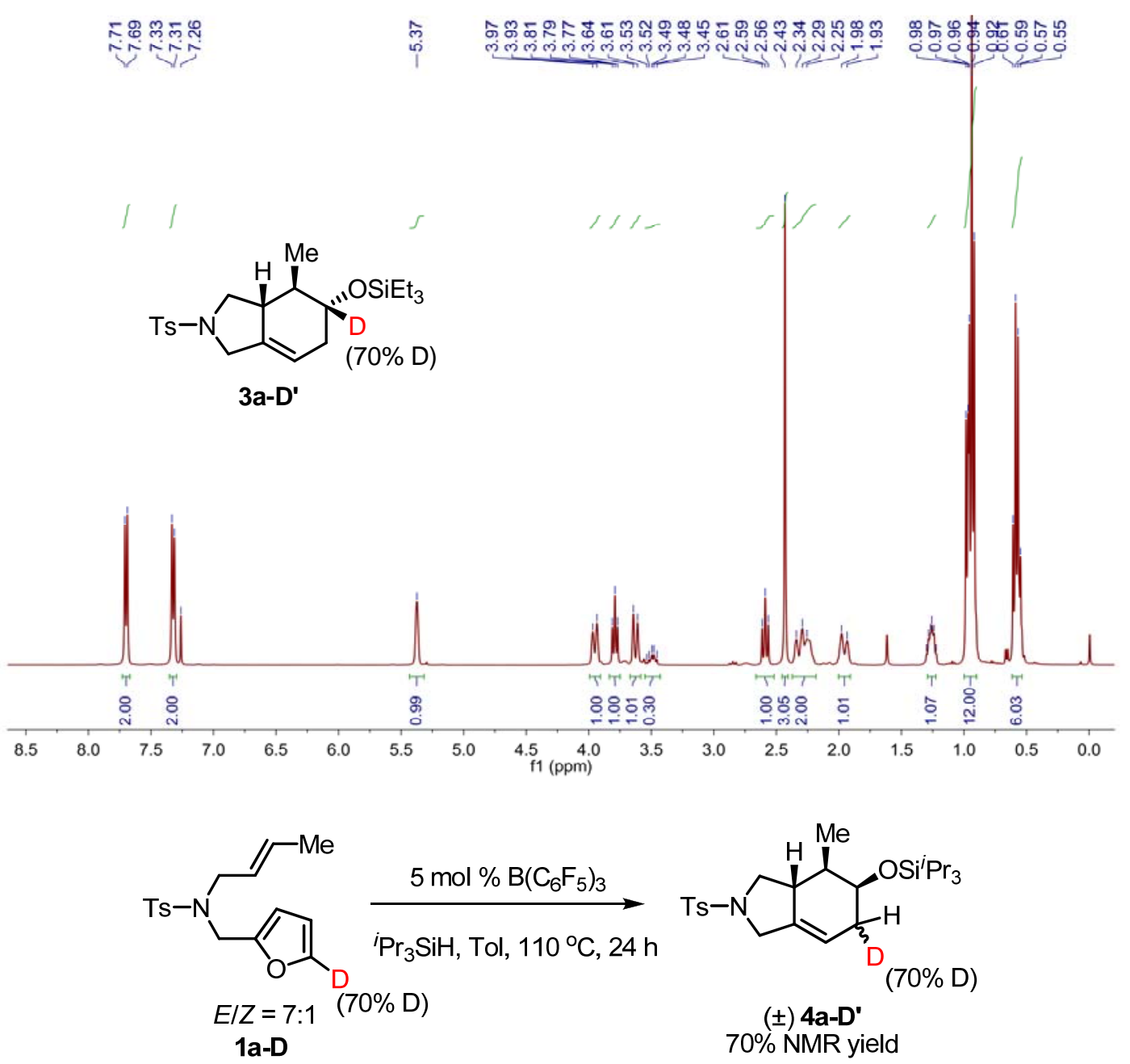

In a glovebox, ${ }^{i} \mathrm{Pr}_{3} \mathrm{SiH}(61 \mathrm{uL}, 0.3 \mathrm{mmol}, 1.5$ equiv) was added to a solution of 1a-D (61 mg, $0.2 \mathrm{mmol}, 1$ equiv) in dry toluene $(0.5 \mathrm{~mL})$ in an $8 \mathrm{~mL}$ vial. $\mathrm{B}\left(\mathrm{C}_{6} \mathrm{~F}_{5}\right)_{3}(5.1 \mathrm{mg}$, $0.01 \mathrm{mmol}, 5 \mathrm{~mol} \%$ ) was then added before the vial was capped and closed tightly. The reaction mixture was stirred at $110{ }^{\circ} \mathrm{C}$ for $24 \mathrm{~h}$. After being allowed to cool to room temperature, one drop of $\mathrm{Et}_{3} \mathrm{~N}$ was added to the solution. After evaporation of the solvent in vacuo, the residue was subjected to column chromatography on silica gel $(\mathrm{PE} / \mathrm{EA}=30: 1$ to $20: 1$ as eluent) to give the product. We did not observe the product from the reaction of the $Z$ isomer of $1 \mathbf{a}-\mathbf{D}$. 


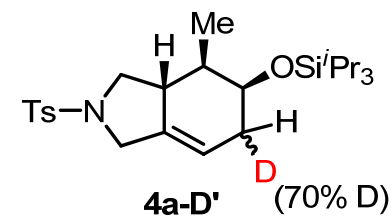

4a-D': White solid. ${ }^{1} \mathrm{H}$ NMR (400 MHz, $\left.\mathrm{CDCl}_{3}\right) \delta 7.72(\mathrm{~d}, J=8.0 \mathrm{~Hz}, 2 \mathrm{H}), 7.31$ (d, $J$ $=8.0 \mathrm{~Hz}, 2 \mathrm{H}), 5.31(\mathrm{~s}, 1 \mathrm{H}), 4.09-3.95(\mathrm{~m}, 2 \mathrm{H}), 3.81(\mathrm{t}, J=8.3 \mathrm{~Hz}, 1 \mathrm{H}), 3.68(\mathrm{~d}, J=$ $13.6 \mathrm{~Hz}, 1 \mathrm{H}), 2.64-2.55(\mathrm{dd}, J=10.9,9.1 \mathrm{~Hz}, 1 \mathrm{H}), 2.54-2.35(\mathrm{~m}, 4 \mathrm{H}), 2.24-2.09$ (m, $1.3 \mathrm{H}), 1.25-1.17(\mathrm{~m}, 1 \mathrm{H}), 1.07-0.95(\mathrm{~m}, 24 \mathrm{H})$.

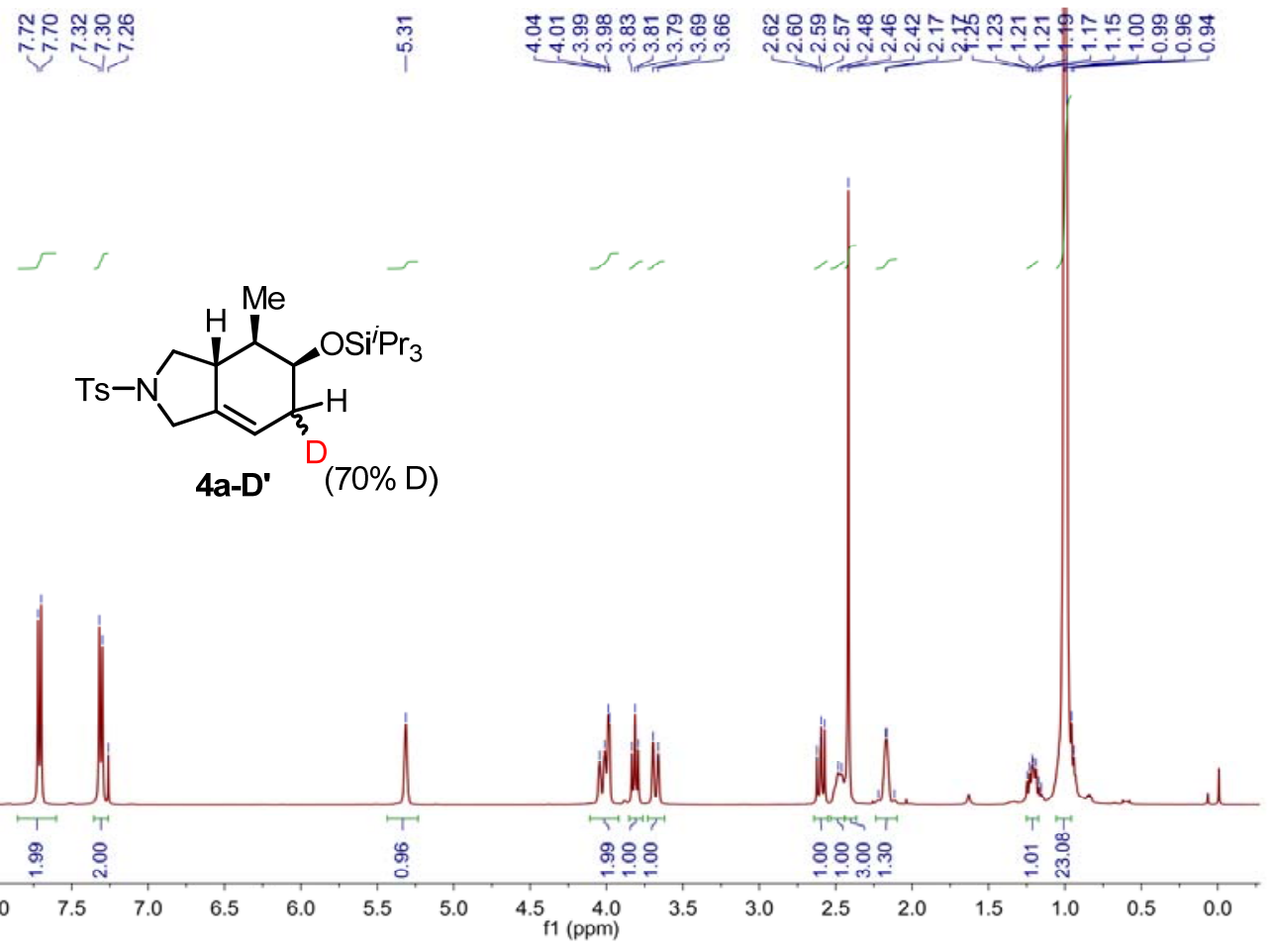

\subsection{Control Experiments}<smiles>[B]N1CC2C=CC(C2)C1OO</smiles>

$( \pm) 2 \mathrm{~h}$

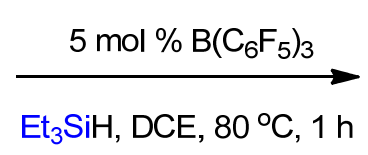

$\mathrm{Et}_{3} \mathrm{SiH}, \mathrm{DCE}, 80^{\circ} \mathrm{C}, 1 \mathrm{~h}$<smiles>[3H]N1CC2=CCC(OCC)CC2C1</smiles>

$( \pm) 3 h$

$83 \%$ NMR yield<smiles>[As]N1CC2C=CC(O2)C1C1CCCC1</smiles>

$( \pm) 2 \mathrm{~h}$

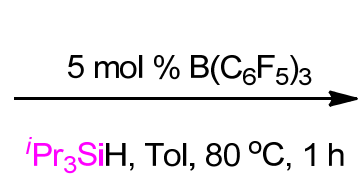

${ }^{i} \mathrm{Pr}_{3} \mathrm{SiH}, \mathrm{Tol}, 80^{\circ} \mathrm{C}, 1 \mathrm{~h}$<smiles>[B]N1CC2=CCC(O[GaH2])CC2C1</smiles>

( \pm ) $4 \mathrm{~h}$

9\% NMR yield $\mathrm{dr}=2.4: 1$<smiles>[B]N1CC2=CCC(=O)C[C@H]2C1</smiles>

( \pm 5

81\% NMR yield 
In a glovebox, ${ }^{i} \mathrm{Pr}_{3} \mathrm{SiH}$ (61 uL, $0.15 \mathrm{mmol}, 1.5$ equiv) or $\mathrm{Et}_{3} \mathrm{SiH}$ (48 uL, $0.15 \mathrm{mmol}$, 1.5 equiv) and $\mathbf{2 h}^{[13]}(29.1 \mathrm{mg}, 0.1 \mathrm{mmol}, 1$ equiv) were dissolved in a dry solvent $(0.25 \mathrm{~mL}$ of toluene or $\mathrm{DCE})$ in an $8 \mathrm{~mL}$ vial. $\mathrm{B}\left(\mathrm{C}_{6} \mathrm{~F}_{5}\right)_{3}(2.6 \mathrm{mg}, 0.005 \mathrm{mmol}, 5$ mol \%) was then added before the vial was capped and closed tightly. The reaction mixture was then stirred at $80{ }^{\circ} \mathrm{C}$ for $1 \mathrm{~h}$. After being allowed to cool to room temperature, one drop of $\mathrm{Et}_{3} \mathrm{~N}$ was added to the solution. After removing all the volatiles, the resulting reaction mixture was analyzed by ${ }^{1} \mathrm{H}$ NMR spectroscopy using $\mathrm{CH}_{2} \mathrm{Br}_{2}$ as the internal standard.<smiles>O=C1CC=C2CN([As])CC2C1</smiles>

5

( \pm )-2-Tosyl-1,2,3,3a,4,6-hexahydro-5H-isoindol-5-one (5). White solid. ${ }^{1} \mathrm{H}$ NMR $\left(400 \mathrm{MHz}_{\mathrm{CDCl}}\right) \delta 7.71(\mathrm{~d}, J=7.9 \mathrm{~Hz}, 2 \mathrm{H}), 7.34(\mathrm{~d}, J=7.9 \mathrm{~Hz}, 2 \mathrm{H}), 5.65(\mathrm{~s}, 1 \mathrm{H})$, $4.08(\mathrm{~d}, J=14.0 \mathrm{~Hz}, 1 \mathrm{H}), 3.88(\mathrm{t}, J=8.6 \mathrm{~Hz}, 1 \mathrm{H}), 3.75(\mathrm{~d}, J=14.0 \mathrm{~Hz}, 1 \mathrm{H}), 2.99-2.75$ (m, 3H), 2.74-2.67 (m, 1H), $2.61(\mathrm{dd}, J=14.5,5.1 \mathrm{~Hz}, 1 \mathrm{H}) 2.44(\mathrm{~s}, 3 \mathrm{H}), 2.14-2.02(\mathrm{~m}$, $1 \mathrm{H}) .{ }^{13} \mathrm{C} \mathrm{NMR}\left(101 \mathrm{MHz}, \mathrm{CDCl}_{3}\right) \delta 208.0,144.0,138.3,132.9,130.0,127.8,116.4$, 54.3, 50.6, 42.4, 38.8, 38.6, 21.7. HRMS (ESI) calcd. for $\mathrm{C}_{15} \mathrm{H}_{18} \mathrm{NO}_{3} \mathrm{~S}^{+}(\mathrm{M}+\mathrm{H})^{+}$: 292.1002, Found: 292.1004.<smiles>[Li]N1CC2C=CC(C2)C1O</smiles>

$( \pm) 2 h$

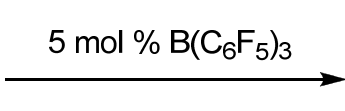

$\mathrm{Tol}, 80^{\circ} \mathrm{C}, 1 \mathrm{~h}$<smiles>[13CH3]N1CC2=CCC(=O)CC2C1</smiles>

( \pm ) 5 $90 \%$ NMR yield

In a glovebox, $\mathrm{B}\left(\mathrm{C}_{6} \mathrm{~F}_{5}\right)_{3}(2.6 \mathrm{mg}, 0.005 \mathrm{mmol}, 5 \mathrm{~mol} \%)$ was added to a solution of $\mathbf{2 h}$ (29.1 mg, $0.1 \mathrm{mmol}, 1$ equiv) in dry toluene $(0.25 \mathrm{~mL})$ before the vial was capped and closed tightly. The reaction mixture was then stirred at $80{ }^{\circ} \mathrm{C}$ for $1 \mathrm{~h}$. After being allowed to cool to room temperature, one drop of $\mathrm{Et}_{3} \mathrm{~N}$ was added to the solution. After evaporation of all the volatiles in vacuo, the resulting mixture was analyzed by ${ }^{1} \mathrm{H}$ NMR spectroscopy using $\mathrm{CH}_{2} \mathrm{Br}_{2}$ as the internal standard.<smiles>O=C1CC=C2CN([13F])CC2C1</smiles>

( \pm ) 5

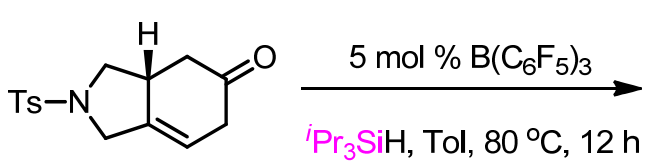

(士) 5<smiles>[3H]N1CC2=CC[C@@H](OCC)C[C@@H]2C1</smiles>

( \pm ) $4 \mathbf{h}^{\prime}$

$99 \%$ NMR yield $\mathrm{dr}=1.6: 1$<smiles>[3H]N1CC2=CC[C@@H](O[AsH3])C[C@H]2C1</smiles>

( \pm ) $4 \mathrm{~h}$ $11 \%$ NMR yield $\mathrm{dr}=2.4: 1$

recovery of starting materials: $73 \%$

In a glovebox, ${ }^{i} \mathrm{Pr}_{3} \mathrm{SiH}\left(31 \mathrm{uL}, 0.15 \mathrm{mmol}, 1.5\right.$ equiv) or $\mathrm{Et}_{3} \mathrm{SiH}(24 \mathrm{uL}, 0.15 \mathrm{mmol}$, 1.5 equiv) was added to a solution of $5(29.1 \mathrm{mg}, 0.1 \mathrm{mmol}, 1$ equiv) in a dry solvent 
$(0.25 \mathrm{~mL}$ of toluene or $\mathrm{DCE})$ in an $8 \mathrm{~mL}$ vial. $\mathrm{B}\left(\mathrm{C}_{6} \mathrm{~F}_{5}\right)_{3}(2.6 \mathrm{mg}, 0.005 \mathrm{mmol}, 5$ mol \%) was then added before the vial was capped and closed tightly. The reaction mixture was stirred at $80{ }^{\circ} \mathrm{C}$. After being allowed to cool to room temperature, one drop of $\mathrm{Et}_{3} \mathrm{~N}$ was added to the solution. After evaporation of all the volatiles in vacuo, the resulting mixture was analyzed by ${ }^{1} \mathrm{H}$ NMR spectroscopy using $\mathrm{CH}_{2} \mathrm{Br}_{2}$ as the internal standard.

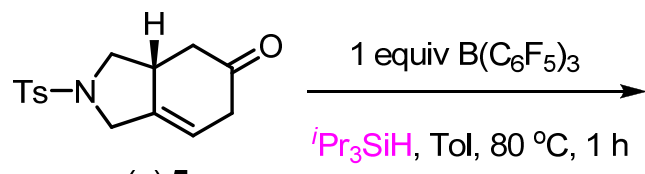

$( \pm) 5$<smiles>[AlH2][C@@H]1CC=C2CN([AsH3-])C[C@@H]2C1</smiles>

$( \pm)$ h

$77 \%$ NMR yield

$\mathrm{dr}=2.4: 1$

In a glovebox, ${ }^{i} \mathrm{Pr}_{3} \mathrm{SiH}(31 \mathrm{uL}, 0.15 \mathrm{mmol}, 1.5$ equiv) was added to a solution of 5 (29.1 mg, $0.1 \mathrm{mmol}, 1$ equiv) in dry toluene $(0.25 \mathrm{~mL})$ in an $8 \mathrm{~mL}$ vial. $\mathrm{B}\left(\mathrm{C}_{6} \mathrm{~F}_{5}\right)_{3}(51.2$ $\mathrm{mg}, 0.1 \mathrm{mmol}, 1$ equiv) was then added before the vial was capped and closed tightly. The reaction mixture was stirred at $80{ }^{\circ} \mathrm{C}$ for $1 \mathrm{~h}$. After being allowed to cool to room temperature, one drop of $\mathrm{Et}_{3} \mathrm{~N}$ was added to the solution. After evaporation of all the volatiles in vacuo, the resulting mixture was analyzed by ${ }^{1} \mathrm{H}$ NMR spectroscopy using $\mathrm{CH}_{2} \mathrm{Br}_{2}$ as the internal standard.

\subsection{DFT studies}

Computational Methods: All quantum chemical calculations were performed with Gaussian 09. ${ }^{[14]}$ Geometrical optimizations and vibrational frequency analysis were calculated with the M06-2X $\mathrm{X}^{[15]}$ density functional and the 6-31G(d) basis set. Intrinsic Reaction Coordinate (IRC) ${ }^{[16,17]}$ calculations were conducted to determine the connectivity of minima and transition states. Single point energy calculations were carried out with the M06-2X density functional, 6-311++G(d,p) basis set, and the SMD solvation mode ${ }^{[18]}$ with toluene as the solvent. Grimme's D3 empirical dispersion was used with M06-2X functional. ${ }^{[19]}$ Gibbs free energies were evaluated at the reaction temperature $(383 \mathrm{~K}$ or $393 \mathrm{~K}$ ) using vibrational frequencies: rigid rotor harmonic oscillator (RRHO) vibrational entropies were used above $100 \mathrm{~cm}^{-1}$, while a free rotor description was used below this value, as described by Grimme. ${ }^{[20]}$ Concentration was corrected from $1 \mathrm{~atm}$ to $1 \mathrm{~mol} / \mathrm{L}$. This was implemented using Goodvibes. ${ }^{[21]}$ The calculated structures were displayed with the CYLview software. ${ }^{[22]}$ 


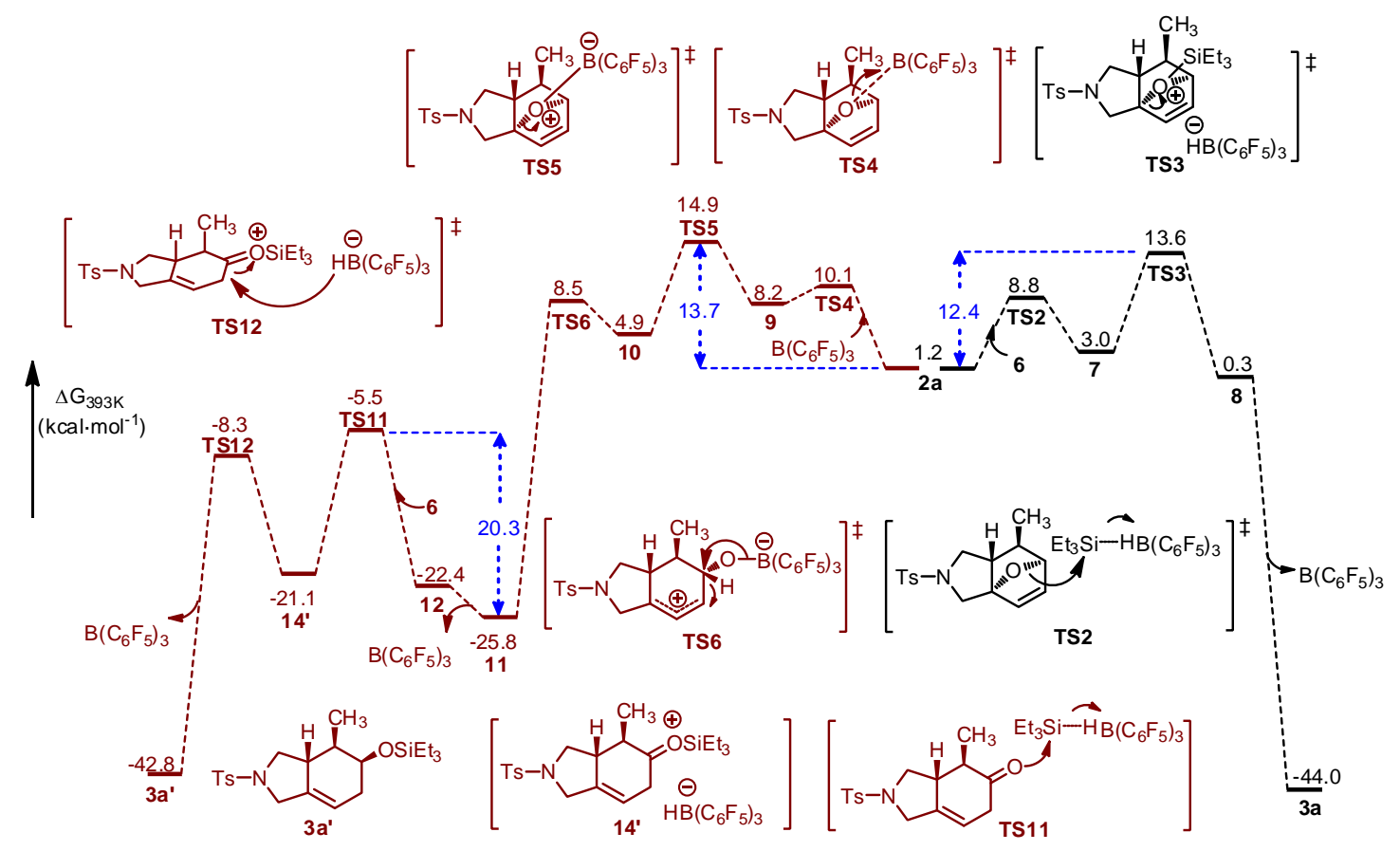

Figure S1. Energy profile $(\mathrm{kcal} / \mathrm{mol})$ for two possible pathways of the reaction of 2a

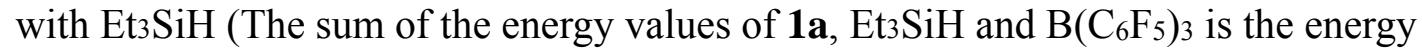
zero point).

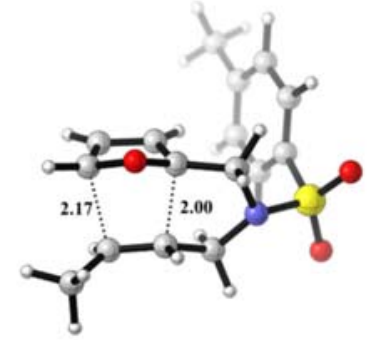

TS1'

IF $=i 607.16 \mathrm{~cm}^{-1}$

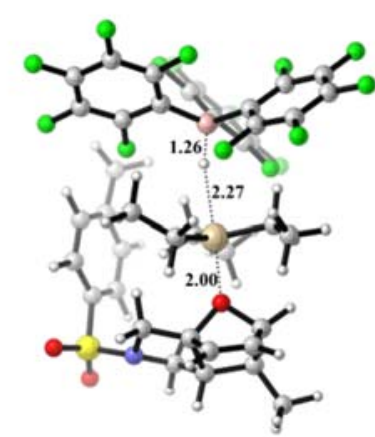

TS2

$\mathrm{IF}=i 53.93 \mathrm{~cm}^{-1}$

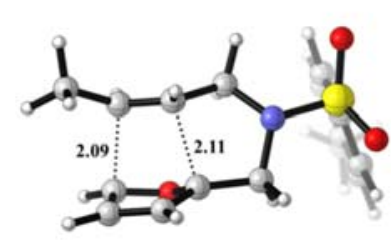

TS1

IF $=i 582.52 \mathrm{~cm}^{-1}$

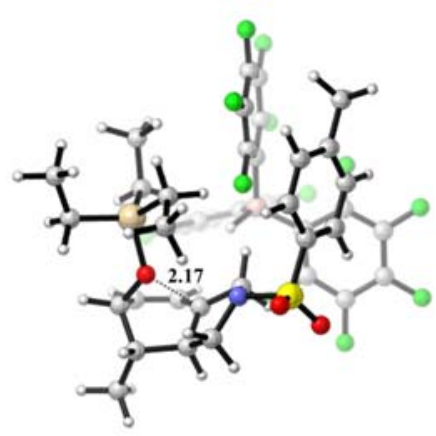

TS3

$\mathrm{IF}=\boldsymbol{i} 220.85 \mathrm{~cm}^{-1}$ 


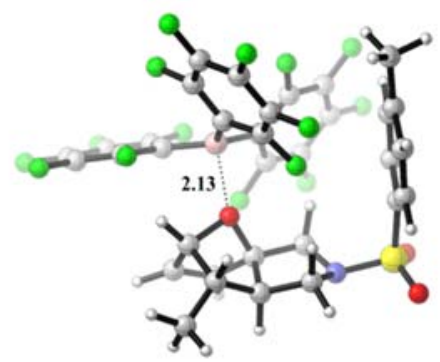

TS4

IF $=\boldsymbol{i} 105.17 \mathrm{~cm}^{-1}$

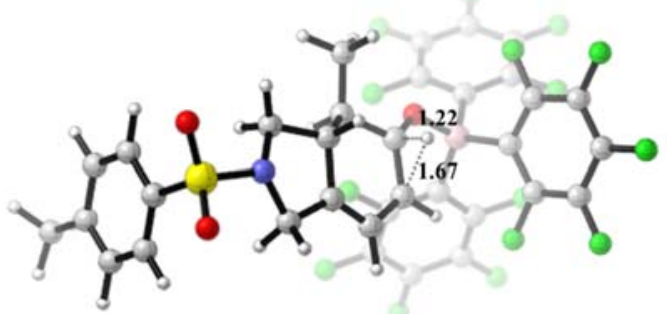

TS6

IF $=i 741.76 \mathrm{~cm}^{-1}$

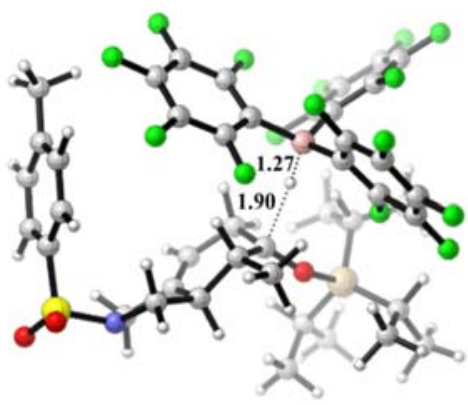

TS8

IF $=i 88.59 \mathrm{~cm}^{-1}$

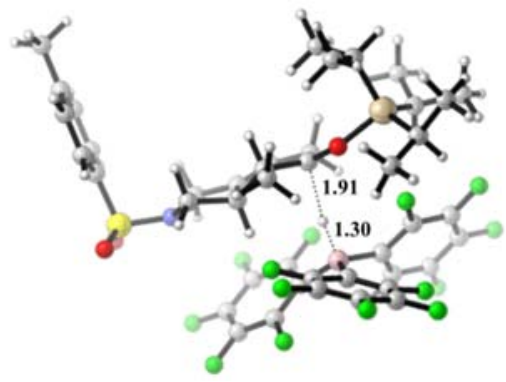

TS10

IF $=i 164.17 \mathrm{~cm}^{-1}$

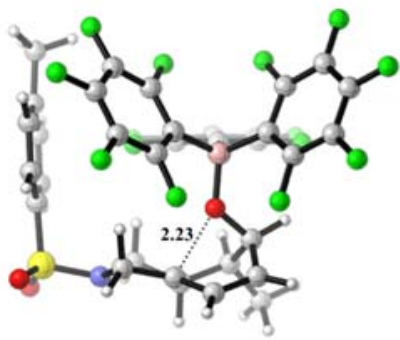

TS5

IF $=i 176.37 \mathrm{~cm}^{-1}$

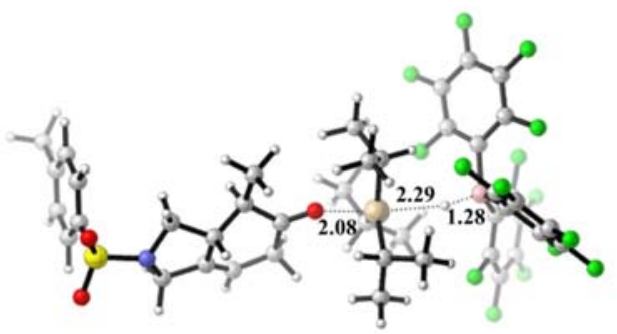

TS7

IF $=i 109.46 \mathrm{~cm}^{-1}$
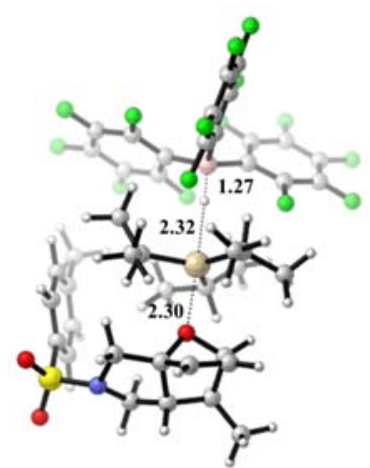

TS9

IF $=i 169.55 \mathrm{~cm}^{-1}$

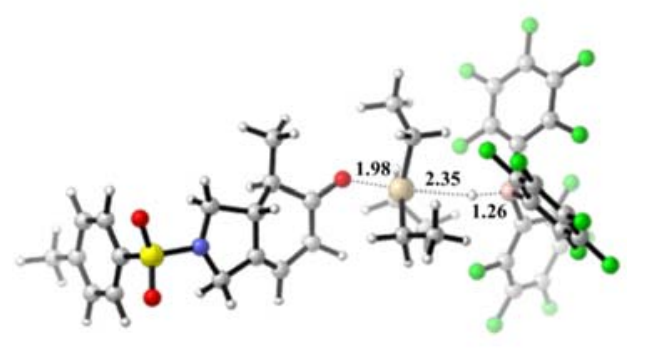

TS11

IF $=i 40.33 \mathrm{~cm}^{-1}$ 


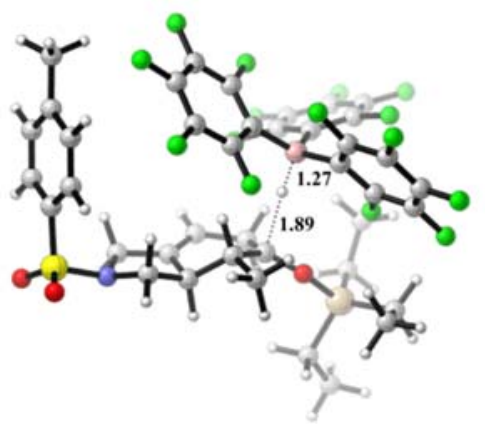

TS12

IF $=i 158.12 \mathrm{~cm}^{-1}$

Figure S2. Optimized transition states and their corresponding imaginary frequencies $\left(\mathrm{IF}, \mathrm{cm}^{-1}\right)$. Bond distances are given in $\AA$.

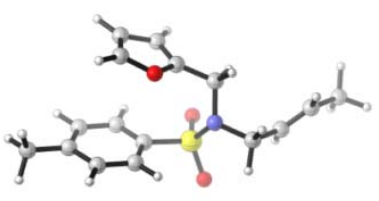

1a

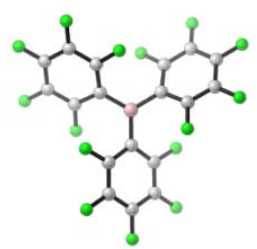

$\mathrm{B}\left(\mathrm{C}_{6} \mathrm{~F}_{5}\right)_{3}$

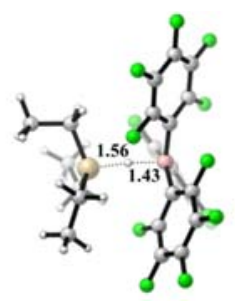

6

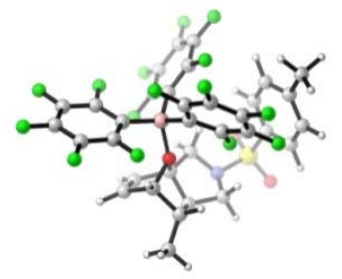

9

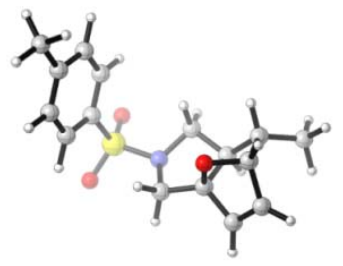

2a

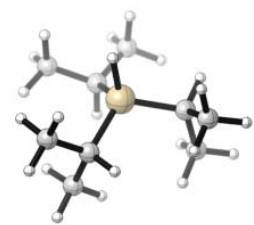

${ }^{i} \mathbf{P r}_{3} \mathrm{SiH}$

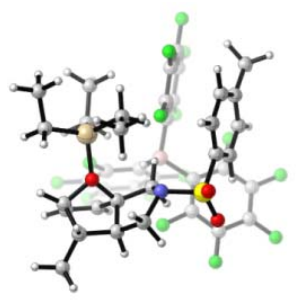

7

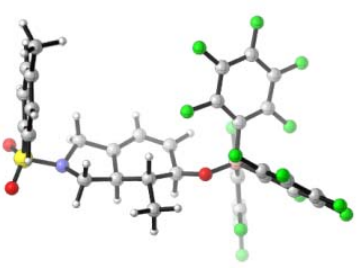

10

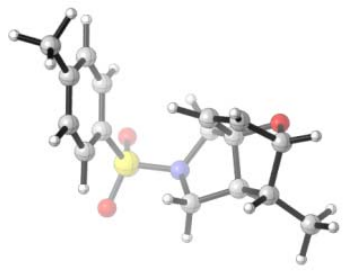

2a'

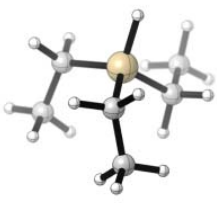

$\mathrm{Et}_{3} \mathrm{SiH}$

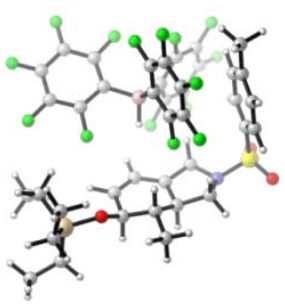

8

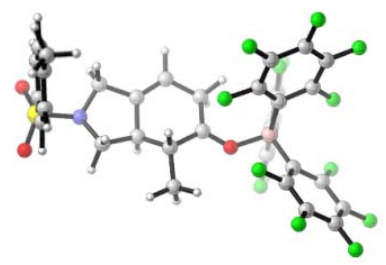

11 


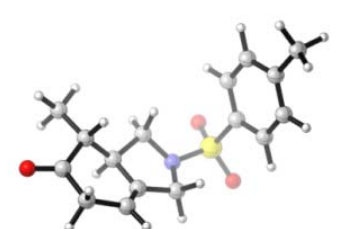

12

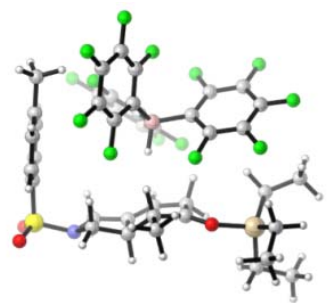

14

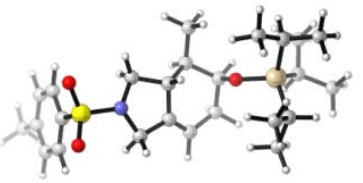

$4 a$

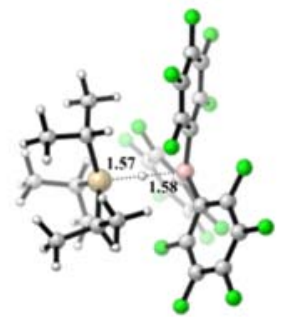

13

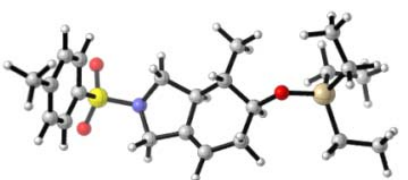

3a

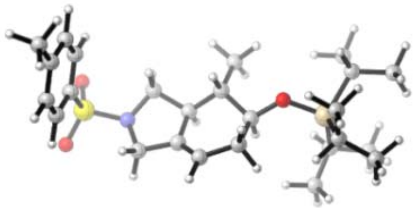

4a'

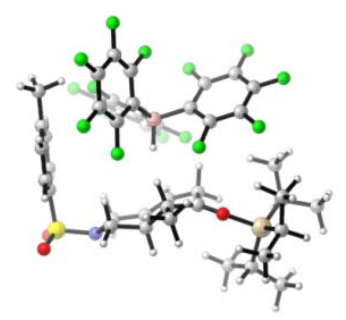

14

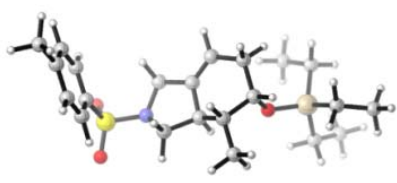

3a'

Figure S3. Optimized structures of starting materials and intermediates. 


\section{Optimized Cartesian coordinates calculated at the M06-2X/6-31G(d) level}

\begin{tabular}{|c|c|c|c|c|c|c|c|}
\hline \multicolumn{4}{|c|}{$\mathrm{B}\left(\mathrm{C}_{6} \mathrm{~F}_{5}\right)_{3}$} & \multicolumn{4}{|c|}{ Et3SiH } \\
\hline B & -0.00049200 & -0.00206900 & -0.00018300 & $\mathrm{Si}$ & 0.02983400 & -0.17970400 & 0.80563700 \\
\hline $\mathrm{C}$ & -1.47156100 & -0.54385300 & 0.00102700 & $\mathrm{H}$ & 0.45450300 & -0.54729100 & 2.18833400 \\
\hline $\mathrm{C}$ & -2.51595700 & 0.13476500 & 0.63751900 & $\mathrm{C}$ & 1.05240100 & -1.19294700 & -0.42038200 \\
\hline $\mathrm{C}$ & -1.82954300 & -1.73673400 & -0.63540100 & $\mathrm{H}$ & 0.68393700 & -1.00187000 & -1.43695900 \\
\hline $\mathrm{C}$ & -3.82129900 & -0.33059800 & 0.65491800 & $\mathrm{H}$ & 0.87391000 & -2.25955500 & -0.23232900 \\
\hline $\mathrm{C}$ & -3.12655800 & -2.22492800 & -0.65356600 & $\mathrm{C}$ & 2.55296600 & -0.88671800 & -0.34264100 \\
\hline $\mathrm{C}$ & -4.12675300 & -1.51681200 & 0.00020100 & $\mathrm{H}$ & 3.13386200 & -1.51630000 & -1.02424600 \\
\hline $\mathrm{C}$ & 0.26461600 & 1.54321100 & 0.00015400 & $\mathrm{H}$ & 2.75727900 & 0.15676200 & 0.60585200 \\
\hline $\mathrm{C}$ & 1.37600200 & 2.10979500 & 0.63252200 & $\mathrm{H}$ & 2.94092800 & -1.05013400 & 0.66867000 \\
\hline $\mathrm{C}$ & -0.59324700 & 2.44858900 & -0.63282500 & $\mathrm{C}$ & 0.37373800 & 1.66222200 & 0.55202600 \\
\hline $\mathrm{C}$ & 1.62402900 & 3.47336900 & 0.64907800 & $\mathrm{H}$ & 1.44376200 & 1.84644000 & 0.71137700 \\
\hline $\mathrm{C}$ & -0.36940500 & 3.81649700 & -0.65151800 & $\mathrm{H}$ & -0.14792200 & 2.22788200 & 1.33426500 \\
\hline $\mathrm{C}$ & 0.74544300 & 4.32987500 & -0.00163700 & $\mathrm{C}$ & -0.04813500 & 2.17429900 & -0.83117300 \\
\hline $\mathrm{C}$ & 1.20542600 & -1.00360100 & -0.00045800 & $\mathrm{H}$ & -1.12876000 & 2.07033300 & -0.97824200 \\
\hline $\mathrm{C}$ & 2.41955300 & -0.71077900 & -0.63049100 & $\mathrm{H}$ & 0.19904200 & 3.23212400 & 0.96734800 \\
\hline $\mathrm{C}$ & 1.14114800 & -2.25049700 & 0.62994200 & $\mathrm{H}$ & 0.44726200 & 1.61623300 & 1.63416100 \\
\hline $\mathrm{C}$ & 3.49386200 & -1.58620500 & -0.64798800 & $\mathrm{C}$ & -1.81720800 & -0.55528600 & 0.59009200 \\
\hline $\mathrm{C}$ & 2.19986500 & -3.14485700 & 0.64785000 & $\mathrm{H}$ & -2.39218400 & 0.31155200 & 0.94158100 \\
\hline $\mathrm{C}$ & 3.38191100 & -2.80951700 & 0.00013200 & $\mathrm{H}$ & -2.09019300 & -1.38448200 & 1.25355900 \\
\hline $\mathrm{F}$ & -1.67869700 & 2.01439100 & -1.27521700 & $\mathrm{C}$ & -2.21885700 & -0.90179100 & -0.85064700 \\
\hline $\mathrm{F}$ & -1.20795300 & 4.63559400 & -1.27708700 & $\mathrm{H}$ & -1.71508000 & -1.81240200 & -1.19045700 \\
\hline $\mathrm{F}$ & 0.97074900 & 5.63406800 & -0.00279800 & $\mathrm{H}$ & -3.29728400 & -1.06828800 & -0.93962400 \\
\hline $\mathrm{F}$ & 2.68874800 & 3.96467400 & 1.27388400 & $\mathrm{H}$ & -1.95016100 & -0.10381800 & -1.55113900 \\
\hline $\mathrm{F}$ & 2.25369700 & 1.33711800 & 1.27432700 & & & & \\
\hline $\mathrm{F}$ & -0.91269200 & -2.45960100 & -1.28061300 & \multicolumn{4}{|c|}{${ }^{i}$ Pr3SiH } \\
\hline $\mathrm{F}$ & -3.41971100 & -3.35764700 & -1.28338400 & $\mathrm{Si}$ & 0.04670300 & -0.04282500 & -0.53058400 \\
\hline $\mathrm{F}$ & -5.36920500 & -1.97274100 & 0.00046000 & $\mathrm{H}$ & -0.16943300 & -0.07035100 & -2.01086100 \\
\hline $\mathrm{F}$ & -4.77718300 & 0.34422600 & 1.28466600 & $\mathrm{C}$ & 1.86168200 & -0.48964700 & -0.20089800 \\
\hline $\mathrm{F}$ & -2.28269400 & 1.27733500 & 1.28540300 & $\mathrm{H}$ & 2.00838200 & -1.49941200 & -0.61098400 \\
\hline $\mathrm{F}$ & 0.03383700 & -2.62755800 & 1.27100100 & $\mathrm{C}$ & 2.82255200 & 0.45660700 & -0.93025700 \\
\hline $\mathrm{F}$ & 2.09474600 & -4.31286600 & 1.27258800 & $\mathrm{H}$ & 2.73199100 & 1.48163200 & -0.55120300 \\
\hline $\mathrm{F}$ & 4.62280200 & -1.26655600 & -1.27161000 & $\mathrm{H}$ & 3.86513900 & 0.14956500 & -0.78172200 \\
\hline $\mathrm{F}$ & 2.58539000 & 0.44729000 & -1.27149100 & $\mathrm{H}$ & 2.63119400 & 0.48234000 & -2.00816900 \\
\hline $\mathrm{F}$ & 4.40008400 & -3.65505600 & 0.00033400 & $\mathrm{C}$ & 2.17604500 & -0.52892100 & 1.29981700 \\
\hline & & & & $\mathrm{H}$ & 1.51330700 & -1.21136000 & 1.84521800 \\
\hline \multicolumn{4}{|c|}{ TS1' } & $\mathrm{H}$ & 3.20840100 & -0.85300700 & 1.48005500 \\
\hline $\mathrm{N}$ & 0.57267700 & -1.62123300 & -0.16117100 & $\mathrm{H}$ & 2.06839600 & 0.46723300 & 1.74694900 \\
\hline $\mathrm{C}$ & 0.96874200 & -0.96036200 & -1.43079900 & $\mathrm{C}$ & -1.10849400 & -1.34004500 & 0.23786900 \\
\hline $\mathrm{C}$ & 2.51450300 & -0.46667300 & 0.56496500 & $\mathrm{H}$ & -1.07887400 & -1.20539500 & 1.32947200 \\
\hline $\mathrm{C}$ & 1.24270700 & -1.09302900 & 1.05912200 & $\mathrm{C}$ & -2.55582900 & -1.14108200 & -0.23054600 \\
\hline $\mathrm{C}$ & 3.24820400 & 0.61570300 & 1.07337200 & $\mathrm{H}$ & -2.62977100 & -1.23279600 & -1.32079200 \\
\hline $\mathrm{C}$ & -1.96902100 & -0.65654400 & 0.10415100 & $\mathrm{H}$ & -3.21940400 & -1.89634900 & 0.20856900 \\
\hline
\end{tabular}




\begin{tabular}{|c|c|c|c|c|c|c|c|}
\hline $\mathrm{C}$ & -2.46617600 & -0.07997400 & -1.06338400 & $\mathrm{H}$ & -2.94899600 & -0.15633500 & 0.04493400 \\
\hline C & -3.11315000 & 1.14758000 & -0.98980500 & C & -0.63094100 & -2.76183600 & -0.08255500 \\
\hline & -3.27494700 & 1.80559000 & 0.23416900 & $\mathrm{H}$ & 0.35716000 & -2.96760200 & 0.34270200 \\
\hline C & -2.78499300 & 1.19615900 & 1.39301300 & $\mathrm{H}$ & -1.32764000 & -3.50900000 & 0.31706800 \\
\hline C & -2.13560400 & -0.03332400 & 1.33885600 & $\mathrm{H}$ & -0.56678200 & -2.92249400 & -1.16613400 \\
\hline & -4.00031200 & 3.12445100 & 0.30139800 & C & -0.34916200 & 1.70733700 & 0.11338100 \\
\hline & -0.99148000 & -2.14171000 & -0.00668100 & $\mathrm{H}$ & 0.60108700 & 2.26180400 & 0.10900300 \\
\hline $\mathrm{O}$ & -1.06307400 & -2.81119500 & 1.28083700 & $\mathrm{C}$ & -0.87124600 & 1.68799100 & 1.55699800 \\
\hline C & -1.34631800 & -2.79423800 & -1.25551600 & $\mathrm{H}$ & -1.01912400 & 2.70790200 & 1.93244600 \\
\hline H & 3.12567500 & -1.18450100 & 0.01188700 & $\mathrm{H}$ & -1.83833900 & 1.17533200 & 1.61922400 \\
\hline $\mathrm{H}$ & 2.77855000 & 1.23206100 & 1.83837400 & $\mathrm{H}$ & -0.18607200 & 1.17701200 & 2.24253300 \\
\hline $\mathrm{H}$ & -2.35870300 & -0.60335400 & -2.00847900 & $\mathrm{C}$ & 58800 & 2.45508700 & -0.80232500 \\
\hline $\mathrm{H}$ & -3.50411000 & 1.60286000 & -1.89599000 & $\mathrm{H}$ & -1.53592400 & 3.46049300 & -0.41562500 \\
\hline $\mathrm{H}$ & -2.92034800 & 1.68841600 & 2.35243500 & $\mathrm{H}$ & -0.93368100 & 2.55851600 & -1.81797100 \\
\hline $\mathrm{H}$ & -1.77547700 & -0.52063300 & 2.23974100 & $\mathrm{H}$ & -2.28697500 & 1.92 & 3900 \\
\hline $\mathrm{H}$ & -3.77220300 & 3.74518300 & -0.56936100 & & & & \\
\hline $\mathrm{H}$ & -5.08437300 & 2.96764400 & 0.31957900 & $1 a$ & & & \\
\hline $\mathrm{H}$ & -3.73040200 & 3.68200900 & 1.20171400 & $\mathrm{C}$ & -0.09212100 & 1.88710200 & -0.17213900 \\
\hline C & 4.75655800 & 0.54165600 & 1.13765800 & 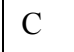 & 0.42332000 & 2.56199500 & 0.888673 \\
\hline $\mathrm{H}$ & 5.15276100 & 0.06551600 & 0.23403700 & $\mathrm{C}$ & 1.76646600 & 2.90501000 & 0.52985300 \\
\hline $\mathrm{H}$ & 5.20973800 & 1.53412400 & 1.22606600 & $\mathrm{C}$ & 1.95562500 & 2.40226900 & -0.71703500 \\
\hline $\mathrm{H}$ & 5.09096800 & -0.05077300 & 1.99792300 & $\mathrm{O}$ & 0.83312000 & 0600 & 13700 \\
\hline $\mathrm{C}$ & 1.57742100 & 0.34023500 & -1.00893400 & $\mathrm{H}$ & -0.08799400 & 2.75701800 & 1.82020200 \\
\hline C & 0.85648800 & 1.45089600 & -0.48634000 & $\mathrm{H}$ & 2.49218800 & 3.43975400 & 1.12440700 \\
\hline $\mathrm{O}$ & 2.77267200 & 0.80287000 & -1.49987300 & $\mathrm{H}$ & 2.79531400 & 2.39093200 & -1.39467700 \\
\hline $\mathrm{C}$ & 1.79491900 & 2.42893900 & -0.27291600 & 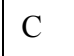 & -1.41800000 & 1.25874800 & -0.41765500 \\
\hline $\mathrm{C}$ & 3.02861900 & 1.86849700 & -0.68231800 & $\mathrm{H}$ & -2.07373900 & 1.48293900 & 0.43174400 \\
\hline $\mathrm{H}$ & 0.09107700 & -0.83659200 & -2.07073900 & $\mathrm{H}$ & -1.87324300 & 1.68174800 & -1.32063600 \\
\hline $\mathrm{H}$ & 1.69585400 & -1.57690200 & -1.96689500 & $\mathrm{~N}$ & -1.32204900 & -0.18993500 & -0.66468200 \\
\hline 2 & 0.62281500 & -0.34405200 & 1.57465000 & $\mathrm{C}$ & -2.56105600 & -0.77686800 & -1.22078100 \\
\hline $\mathrm{H}$ & 1.40652500 & -1.93561000 & 1.73462100 & $\mathrm{H}$ & -2.73858100 & -0.25834300 & -2.16956400 \\
\hline $\mathrm{H}$ & 3.96649200 & 2.37605700 & -0.87139800 & $\mathrm{H}$ & -2.34846400 & -1.82467600 & -1.43928700 \\
\hline $\mathrm{H}$ & 1.67379800 & 3.37659300 & 0.23401700 & $\mathrm{C}$ & -3.75410700 & -0.64498000 & -0.31613500 \\
\hline \multirow[t]{2}{*}{$\mathrm{H}$} & -0.18367500 & 1.43964900 & -0.18391200 & $\mathrm{C}$ & -4.80675300 & 0.12443900 & -0.58740300 \\
\hline & & & & $\mathrm{H}$ & -3.71568900 & -1.21464800 & 0.61348600 \\
\hline $\mathbf{0}$ & & & & $\mathrm{H}$ & -4.81680200 & 0.68488800 & -1.52470200 \\
\hline $\mathrm{Si}$ & 0.02273700 & -0.21319100 & 2.49838000 & C & -6.00835100 & 0.27880300 & 0.29535100 \\
\hline $\mathrm{C}$ & 1.86453400 & -0.38457500 & 2.85886300 & $\mathrm{H}$ & -6.16128400 & 1.32825600 & 0.57050400 \\
\hline $\mathrm{H}$ & 2.40866500 & -0.05740800 & 1.96434900 & $\mathrm{H}$ & -6.91791100 & -0.05164200 & -0.21866800 \\
\hline $\mathrm{H}$ & 2.10513100 & 0.35881600 & 3.63029200 & $\mathrm{H}$ & -5.90101700 & -0.30484800 & 1.21333800 \\
\hline $\mathrm{C}$ & 2.34442600 & -1.77402300 & 3.29897900 & S & -0.64888500 & -1.06343600 & 0.61098000 \\
\hline 11 & 3.42346200 & -1.77185800 & 3.47948200 & $\mathrm{O}$ & -0.92871700 & -0.40542700 & 1.88273000 \\
\hline $\mathrm{H}$ & 1.85794100 & -2.09115700 & 4.22698600 & $\mathrm{O}$ & -1.05477900 & -2.44721100 & 0.40020100 \\
\hline $\mathrm{H}$ & 2.13681500 & -2.53241800 & 2.53966200 & $\mathrm{C}$ & 1.09509500 & -0.93612400 & 0.29369800 \\
\hline
\end{tabular}




\begin{tabular}{|rrrr}
$\mathrm{C}$ & -1.02472900 & -1.74905700 & 2.80133600 \\
$\mathrm{H}$ & -1.00978300 & -2.34723200 & 1.88207400 \\
$\mathrm{H}$ & -0.50385300 & -2.35732900 & 3.55267000 \\
$\mathrm{C}$ & -2.47166000 & -1.48985400 & 3.24440100 \\
$\mathrm{H}$ & -2.99957200 & -2.43424900 & 3.40671400 \\
$\mathrm{H}$ & -2.51212300 & -0.92643600 & 4.18189600 \\
$\mathrm{H}$ & -3.03267100 & -0.92157300 & 2.49854500 \\
$\mathrm{C}$ & -0.71359400 & 1.36878900 & 3.18687400 \\
$\mathrm{H}$ & -0.19479000 & 2.22525300 & 2.74084400 \\
$\mathrm{H}$ & -1.76336100 & 1.43593000 & 2.87966300 \\
$\mathrm{C}$ & -0.59151000 & 1.42641300 & 4.71910600 \\
$\mathrm{H}$ & -1.04370500 & 2.34047600 & 5.11408500 \\
$\mathrm{H}$ & -1.09112600 & 0.57959300 & 5.20153200 \\
$\mathrm{H}$ & 0.45559400 & 1.41584100 & 5.03780100 \\
$\mathrm{H}$ & -0.03750800 & -0.00512500 & 0.95221200 \\
$\mathrm{~B}$ & 0.00538200 & 0.07599600 & -0.47932900 \\
$\mathrm{C}$ & -0.26626600 & 1.64581000 & -0.61904500 \\
$\mathrm{C}$ & 0.53253600 & 2.55504200 & 0.06845400 \\
$\mathrm{C}$ & -1.28771400 & 2.20627200 & -1.37857400 \\
$\mathrm{C}$ & 0.34830400 & 3.92653600 & 0.02941400 \\
$\mathrm{C}$ & -1.50260800 & 3.57795500 & -1.45766800 \\
$\mathrm{~F}$ & 1.52984800 & 2.09502500 & 0.84777500 \\
$\mathrm{~F}$ & -0.67932200 & 4.44169000 & -0.75144900 \\
$\mathrm{C}$ & 1.49945400 & -0.44401100 & -0.72165000 \\
$\mathrm{C}$ & -0.87437100 & 5.75307400 & -0.81483100 \\
$\mathrm{~F}$ & -2.90039800 & -1.66733000 & -0.19158800 \\
$\mathrm{C}$ & 2.49650400 & 0.26838600 & -1.38140200 \\
$\mathrm{C}$ & 3.18981300 & -2.16315800 & -0.27007300 \\
$\mathrm{C}$ & 3.80200900 & -0.19793000 & -1.50242500 \\
$\mathrm{C}$ & 4.15134800 & -1.41690600 & -0.94100300 \\
$\mathrm{C}$ & -1.20210100 & -0.92464600 & -0.79229900 \\
$\mathrm{C}$ & -2.43874600 & -0.71378100 & -0.18850400 \\
$\mathrm{C}$ & -1.11704700 & -2.06239700 & -1.58807900 \\
$\mathrm{C}$ & -3.52016800 & -1.56709800 & -0.32291000 \\
$\mathrm{C}$ & -2.18288900 & -2.93802400 & -1.76824900 \\
$\mathrm{C}$ & -3.38897200 & -2.69169800 & -1.12875000 \\
$\mathrm{~F}$ & 0.01714100 & -2.36891100 & -2.22516700 \\
\hline & -2.05193500 & -4.01173400 & -2.54202600 \\
$\mathrm{~F}$ & -4628400 & -3.52511600 & -1.28280300 \\
\hline & -1.32415900 & 0.31320500 \\
\hline
\end{tabular}

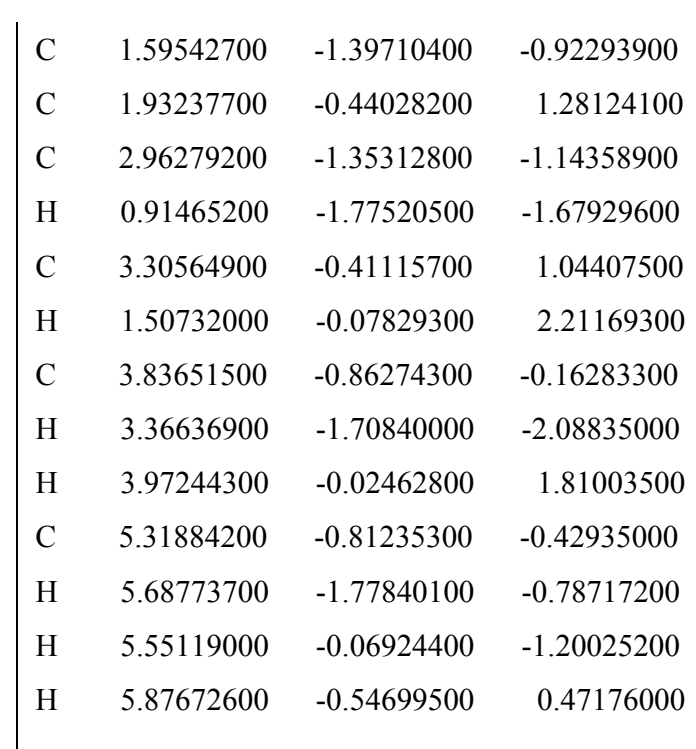

TS1

$\begin{array}{llll}\text { C } & 2.09865200 & -0.08451300 & -1.10349500\end{array}$

$\begin{array}{llll}\text { C } & 3.41668100 & 0.10984000 & -1.58097200\end{array}$

$\begin{array}{llll}\text { C } & 4.11164200 & -1.02730100 & -1.23867500\end{array}$

$\begin{array}{llll}\text { C } & 3.18489300 & -1.85057300 & -0.55223100\end{array}$

$\begin{array}{llll}\mathrm{O} & 1.92437200 & -1.41945900 & -0.86354300\end{array}$

$\begin{array}{llll}\mathrm{H} & 3.79984300 & 1.03528300 & -1.98917300\end{array}$

$\mathrm{H} \quad 5.17425800 \quad-1.20782500 \quad-1.32179300$

H $\quad 3.27135000 \quad-2.91126000 \quad-0.35093700$

$\begin{array}{llll}\text { C } & 0.84000100 & 0.69796100 & -1.30622300\end{array}$

$\begin{array}{llll}\mathrm{N} & 0.46475200 & 1.27388300 & -0.00041300\end{array}$

$\begin{array}{llll}\text { C } & 0.94812000 & 0.48008300 & 1.14037900\end{array}$

$\begin{array}{llll}\text { S } & -1.05399900 & 1.93992500 & 0.09543900\end{array}$

$\begin{array}{llll}\mathrm{O} & -1.15018200 & 2.56523400 & 1.40314100\end{array}$

$\begin{array}{llll}\mathrm{O} & -1.24063100 & 2.68394500 & -1.13986300\end{array}$

$\begin{array}{llll}\text { C } & -2.18973400 & 0.56913900 & 0.07235800\end{array}$

$\begin{array}{llll}\text { C } & -2.49749300 & -0.07863800 & 1.26771400\end{array}$

$\begin{array}{lrrr}\text { C } & -2.71323800 & 0.12871200 & -1.13973500\end{array}$

$\begin{array}{llll}\text { C } & -3.33614000 & -1.18522600 & 1.23822500\end{array}$

$\begin{array}{llll}\mathrm{H} & -2.10026100 & 0.30230100 & 2.20373500\end{array}$

$\begin{array}{llll}\text { C } & -3.55201400 & -0.98168100 & -1.14918700\end{array}$

$\begin{array}{llll}\mathrm{H} & -2.48070600 & 0.66927800 & -2.05208200\end{array}$

$\begin{array}{llll}\text { C } & -3.87238000 & -1.65243100 & 0.03309800\end{array}$

$\begin{array}{llll}\mathrm{H} & -3.58636900 & -1.69422600 & 2.16546400\end{array}$

$\mathrm{H} \quad-3.96891000 \quad-1.33058600 \quad-2.09009100$

$\begin{array}{llll}\text { C } & -4.80304200 & -2.83743800 & 0.02334300\end{array}$

$\begin{array}{llll}\mathrm{H} & -5.80942100 & -2.54070300 & 0.33847800\end{array}$

$\mathrm{H} \quad-4.88190200 \quad-3.27347100 \quad-0.97550800$

$\begin{array}{llll}\mathrm{H} & -4.45942700 & -3.61480300 & 0.71159400\end{array}$

$\begin{array}{llll}\text { C } & 2.42675500 & 0.27773700 & 0.95004400\end{array}$ 


\begin{tabular}{|c|c|c|c|}
\hline $\mathrm{F}$ & 1.00568600 & -2.40818400 & 0.48845000 \\
\hline $\mathrm{F}$ & 3.51080100 & -3.32678600 & 0.29086300 \\
\hline $\mathrm{F}$ & 5.39533300 & -1.86764800 & -1.04157000 \\
\hline $\mathrm{F}$ & 4.71663500 & 0.51871000 & -2.14937900 \\
\hline $\mathrm{F}$ & 2.23703500 & 1.45333400 & -1.94111700 \\
\hline 7 & & & \\
\hline $\mathrm{N}$ & 3.20226000 & -0.51024600 & 1.36802300 \\
\hline $\mathrm{C}$ & 1.92160900 & 0.14344800 & 1.05762200 \\
\hline C & 2.31204400 & 1.58099300 & 0.98565000 \\
\hline $\mathrm{C}$ & 3.35206600 & 1.78404900 & 2.11478000 \\
\hline $\mathrm{C}$ & 4.03490400 & 0.40244000 & 2.20773100 \\
\hline $\mathrm{C}$ & 1.36922700 & 2.74946900 & 0.89515400 \\
\hline$c$ & 2.08879900 & 3.78743100 & 0.46406600 \\
\hline $\mathrm{C}$ & 3.50408100 & 3.29241900 & 0.25561000 \\
\hline $\mathrm{C}$ & 4.19651200 & 2.98225400 & 1.61227100 \\
\hline $\mathrm{O}$ & 3.24635600 & 1.89594900 & -0.17120500 \\
\hline $\mathrm{C}$ & 4.24962400 & 4.17637700 & 2.55539800 \\
\hline $\mathrm{C}$ & 2.02471800 & -2.86869600 & 0.87874600 \\
\hline $\mathrm{C}$ & 0.65945600 & -2.99270400 & 1.11377000 \\
\hline $\mathrm{C}$ & -0.12635500 & -3.63706500 & 0.16373000 \\
\hline $\mathrm{C}$ & 0.43618500 & -4.14177500 & -1.01120700 \\
\hline $\mathrm{C}$ & 1.81230800 & -3.98839700 & -1.22499800 \\
\hline $\mathrm{C}$ & 2.61827900 & -3.36804500 & -0.27924200 \\
\hline $\mathrm{C}$ & -0.42140100 & -4.86548000 & -2.01616100 \\
\hline S & 3.02216600 & -2.03827400 & 2.08203900 \\
\hline $\mathrm{O}$ & 2.24294400 & -1.89549700 & 3.30754800 \\
\hline $\mathrm{O}$ & 4.36223300 & -2.60008800 & 2.12454300 \\
\hline $\mathrm{H}$ & 1.19262900 & 0.06277100 & 1.87524700 \\
\hline $\mathrm{H}$ & 1.46788100 & -0.25298600 & 0.15137100 \\
\hline $\mathrm{H}$ & 2.82385100 & 2.01983900 & 3.04257400 \\
\hline $\mathrm{H}$ & 4.07347200 & 0.06054000 & 3.24696200 \\
\hline $\mathrm{H}$ & 5.05300100 & 0.41138100 & 1.80962100 \\
\hline $\mathrm{H}$ & 0.33192000 & 2.70197300 & 1.20015100 \\
\hline $\mathrm{H}$ & 1.76874800 & 4.81274800 & 0.33153400 \\
\hline H & 4.12287800 & 3.79955800 & -0.48224900 \\
\hline $\mathrm{H}$ & 5.21140100 & 2.63944500 & 1.38363700 \\
\hline $\mathrm{H}$ & 4.77070000 & 3.90597800 & 3.47746400 \\
\hline $\mathrm{H}$ & 4.78373300 & 5.01787300 & 2.10275000 \\
\hline $\mathrm{H}$ & 3.24221900 & 4.50899200 & 2.82257300 \\
\hline $\mathrm{H}$ & 0.23138200 & -2.59933800 & 2.03140100 \\
\hline $\mathrm{H}$ & -1.19455900 & -3.74320600 & 0.32913200 \\
\hline $\mathrm{H}$ & 2.25398700 & -4.37389200 & -2.14002200 \\
\hline $\mathrm{H}$ & 3.69136400 & -3.27284200 & -0.42103100 \\
\hline
\end{tabular}

\begin{tabular}{|c|c|c|c|}
\hline $\mathrm{H}$ & 3.00086100 & 1.20248100 & 0.92438600 \\
\hline $\mathrm{C}$ & 3.09291500 & -0.90112100 & 1.30891100 \\
\hline $\mathrm{H}$ & 2.47677000 & -1.69745300 & 1.72692000 \\
\hline $\mathrm{C}$ & 4.51652800 & -0.85039000 & 1.80895200 \\
\hline $\mathrm{H}$ & 4.55725300 & -0.58502600 & 2.87166200 \\
\hline $\mathrm{H}$ & 5.02613600 & -1.81228600 & 1.68652800 \\
\hline $\mathrm{H}$ & 5.08468100 & -0.09443200 & 1.25692700 \\
\hline $\mathrm{H}$ & 0.44025500 & -0.49658400 & 1.19789900 \\
\hline $\mathrm{H}$ & 0.72414500 & 1.05093500 & 2.04567700 \\
\hline $\mathrm{H}$ & 0.06687700 & 0.01164300 & -1.68267000 \\
\hline $\mathrm{H}$ & 0.97394300 & 1.50804900 & -2.02407000 \\
\hline \multicolumn{4}{|c|}{$2 a$} \\
\hline $\mathrm{C}$ & 2.40603500 & 0.34206700 & 0.61546300 \\
\hline $\mathrm{C}$ & 2.08090800 & -0.01341000 & -0.87799100 \\
\hline $\mathrm{C}$ & 3.38926600 & -0.08568800 & -1.63824900 \\
\hline $\mathrm{C}$ & 3.96995400 & -1.20869700 & -1.21356300 \\
\hline $\mathrm{C}$ & 2.99454200 & -1.81417100 & -0.21242200 \\
\hline $\mathrm{C}$ & 3.04978400 & -0.97066500 & 1.11147200 \\
\hline $\mathrm{C}$ & 1.01596500 & 0.69966900 & 1.13657500 \\
\hline $\mathrm{N}$ & 0.44628300 & 1.42505200 & -0.01060700 \\
\hline $\mathrm{C}$ & 0.88733900 & 0.82801100 & -1.28684000 \\
\hline $\mathrm{O}$ & 1.73715600 & -1.39478100 & -0.73787600 \\
\hline $\mathrm{C}$ & -2.15427300 & 0.55916500 & 0.06892500 \\
\hline $\mathrm{C}$ & -2.43465300 & -0.09719900 & 1.26690800 \\
\hline $\mathrm{C}$ & -3.20610200 & -1.25126000 & 1.23874600 \\
\hline $\mathrm{C}$ & -3.70203700 & -1.75885000 & 0.03226000 \\
\hline $\mathrm{C}$ & -3.41737700 & -1.07492300 & -1.15099800 \\
\hline $\mathrm{C}$ & -2.64530800 & 0.08311500 & -1.14296600 \\
\hline $\mathrm{C}$ & -4.52520800 & -3.02093600 & 0.02189900 \\
\hline $\mathrm{S}$ & -1.10425300 & 1.99508800 & 0.08880500 \\
\hline $\mathrm{O}$ & -1.34147500 & 2.72702600 & -1.14451000 \\
\hline $\mathrm{O}$ & -1.22708100 & 2.60840700 & 1.40031200 \\
\hline $\mathrm{C}$ & 4.43346200 & -0.82255100 & 1.72921600 \\
\hline $\mathrm{H}$ & 3.07395400 & 1.20554600 & 0.68953300 \\
\hline $\mathrm{H}$ & 2.37723700 & -1.46060100 & 1.82447300 \\
\hline $\mathrm{H}$ & 1.01339400 & 1.34999300 & 2.01215300 \\
\hline $\mathrm{H}$ & 0.44863100 & -0.21811800 & 1.35513000 \\
\hline $\mathrm{H}$ & -2.07234000 & 0.31612300 & 2.20327100 \\
\hline $\mathrm{H}$ & -3.43799400 & -1.76507800 & 2.16821000 \\
\hline $\mathrm{H}$ & -3.81169000 & -1.44912200 & -2.09174500 \\
\hline $\mathrm{H}$ & -2.44431000 & 0.63396500 & -2.05659700 \\
\hline $\mathrm{H}$ & -3.90252900 & -3.89220100 & 0.25151500 \\
\hline $\mathrm{H}$ & -5.31899000 & -2.97840700 & 0.77375900 \\
\hline
\end{tabular}




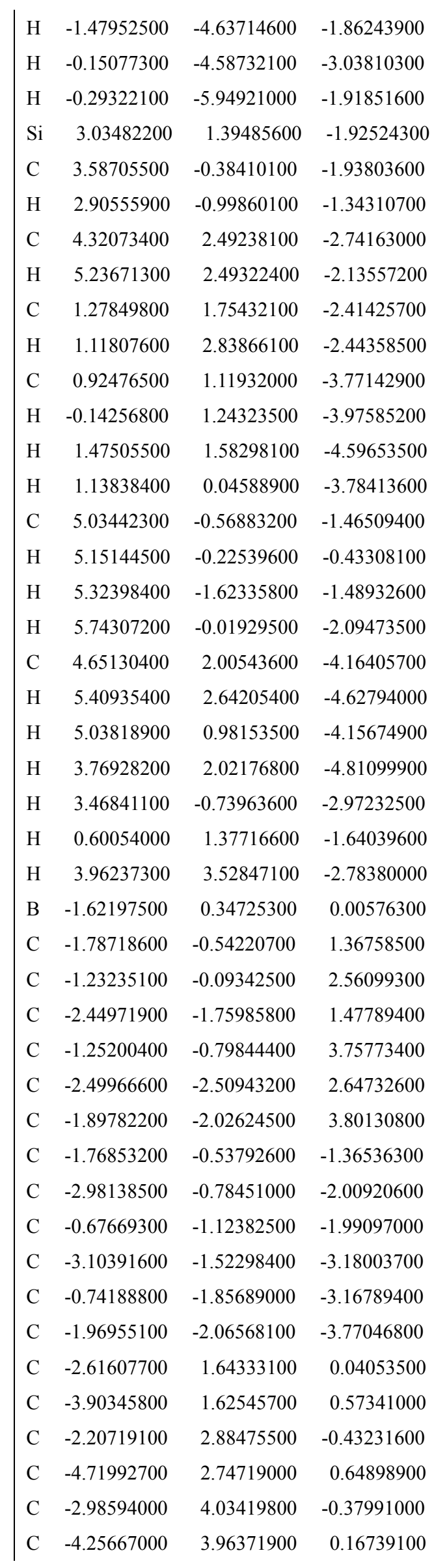

\begin{tabular}{|c|c|c|c|}
\hline $\mathrm{H}$ & -4.98753500 & -3.18613900 & -0.95409000 \\
\hline $\mathrm{H}$ & 4.38444000 & -0.26171500 & 2.66732200 \\
\hline $\mathrm{H}$ & 4.87621700 & -1.80061200 & 1.94709700 \\
\hline $\mathrm{H}$ & 5.10737000 & -0.28606200 & 1.05367300 \\
\hline $\mathrm{H}$ & 0.12569700 & 0.17548900 & -1.73376200 \\
\hline $\mathrm{H}$ & 1.13916500 & 1.62100300 & -1.99390900 \\
\hline $\mathrm{H}$ & 3.78667500 & 0.68761300 & -2.28432400 \\
\hline $\mathrm{H}$ & 4.96243400 & -1.58020400 & -1.43667500 \\
\hline $\mathrm{H}$ & 3.02167300 & -2.89551600 & -0.07568200 \\
\hline \multicolumn{4}{|c|}{$2 a^{\prime}$} \\
\hline $\mathrm{C}$ & 2.41937800 & -0.35134600 & 0.37386100 \\
\hline $\mathrm{C}$ & 1.71468900 & 0.26151300 & -0.86246000 \\
\hline $\mathrm{C}$ & 0.86675600 & 1.45135900 & -0.42471800 \\
\hline $\mathrm{C}$ & 1.74140600 & 2.42076000 & -0.16577400 \\
\hline $\mathrm{C}$ & 3.09783300 & 1.78133600 & -0.39398000 \\
\hline $\mathrm{C}$ & 3.31036500 & 0.81001200 & 0.84479000 \\
\hline $\mathrm{C}$ & 1.25887400 & -1.04820300 & 1.05758300 \\
\hline $\mathrm{N}$ & 0.56718200 & -1.60099300 & -0.14712500 \\
\hline $\mathrm{C}$ & 0.96636900 & -0.93028600 & -1.41958200 \\
\hline $\mathrm{O}$ & 2.82755600 & 0.88438800 & -1.48793700 \\
\hline $\mathrm{C}$ & -1.98118000 & -0.66721500 & 0.10702200 \\
\hline $\mathrm{C}$ & -2.49300200 & -0.12391100 & -1.07018000 \\
\hline $\mathrm{C}$ & -3.15586100 & 1.09657000 & -1.02020700 \\
\hline $\mathrm{C}$ & -3.31917800 & 1.77967500 & 0.18959900 \\
\hline $\mathrm{C}$ & -2.81378500 & 1.20317900 & 1.35877700 \\
\hline $\mathrm{C}$ & -2.14859600 & -0.01836300 & 1.32837100 \\
\hline $\mathrm{C}$ & -4.06272500 & 3.08937300 & 0.23157300 \\
\hline $\mathrm{S}$ & -0.98529700 & -2.14098100 & 0.02154700 \\
\hline $\mathrm{O}$ & -1.04220300 & -2.78737700 & 1.32178300 \\
\hline $\mathrm{O}$ & -1.33179100 & -2.81970000 & -1.21504300 \\
\hline $\mathrm{C}$ & 4.77031500 & 0.40787000 & 1.01670300 \\
\hline $\mathrm{H}$ & 3.07740500 & -1.13173000 & -0.02891600 \\
\hline $\mathrm{H}$ & 2.93711500 & 1.28938500 & 1.75494600 \\
\hline $\mathrm{H}$ & 1.52661800 & -1.86884900 & 1.72319600 \\
\hline $\mathrm{H}$ & 0.62134700 & -0.33466900 & 1.59938500 \\
\hline $\mathrm{H}$ & 0.08889400 & -0.66568700 & -2.01513200 \\
\hline $\mathrm{H}$ & 1.61128400 & -1.58855200 & -2.00805700 \\
\hline $\mathrm{H}$ & -2.38349800 & -0.66775600 & -2.00342400 \\
\hline $\mathrm{H}$ & -3.55853800 & 1.52569100 & -1.93400000 \\
\hline $\mathrm{H}$ & -2.94980200 & 1.71531500 & 2.30761300 \\
\hline $\mathrm{H}$ & -1.77710800 & -0.48016200 & 2.23802800 \\
\hline $\mathrm{H}$ & -3.84754000 & 3.69391700 & -0.65375700 \\
\hline $\mathrm{H}$ & -5.14435700 & 2.91773100 & 0.25859800 \\
\hline
\end{tabular}




\begin{tabular}{|c|c|c|c|c|c|c|c|}
\hline $\mathrm{H}$ & -0.48844700 & 0.78495400 & 0.02208200 & $\mathrm{H}$ & -3.79634900 & 3.6701600 & 1.11811200 \\
\hline $\mathrm{F}$ & -4.12105800 & -0.32052400 & -1.48776200 & $\mathrm{H}$ & 5.38461200 & 1.2674430 & 1.30287400 \\
\hline $\mathrm{F}$ & -4.29630800 & -1.72499600 & -3.74036200 & $\mathrm{H}$ & 4.88490100 & -0.3617680 & 1.78627000 \\
\hline $\mathrm{F}$ & -2.05902700 & -2.78474700 & -4.88824600 & $\mathrm{H}$ & 5.16098000 & 0.0080680 & 0.07481600 \\
\hline $\mathrm{F}$ & 0.37619100 & -2.36057900 & -3.71341500 & $\mathrm{H}$ & 3.94421500 & 2.4257970 & -0.63279200 \\
\hline $\mathrm{F}$ & 0.57322400 & -1.00861200 & -1.47906100 & $\mathrm{H}$ & -0.21169800 & 1.4464970 & -0.30236500 \\
\hline $\mathrm{F}$ & -0.59053600 & 1.10022900 & 2.59965100 & $\mathrm{H}$ & 1.56755400 & 3.4093810 & 0.24104800 \\
\hline $\mathrm{F}$ & -0.68045600 & -0.30105200 & 4.85352100 & & & & \\
\hline $\mathrm{F}$ & -1.94954000 & -2.72291300 & 4.93308300 & \multicolumn{4}{|c|}{ TS2 } \\
\hline $\mathrm{F}$ & -3.11222900 & -3.69404500 & 2.66246700 & $\mathrm{C}$ & -5.03739300 & 2.29788900 & 0.13895500 \\
\hline $\mathrm{F}$ & -3.05747300 & -2.31143100 & 0.41394700 & $\mathrm{C}$ & -3.91481800 & 2.07495500 & -0.92358900 \\
\hline $\mathrm{F}$ & -0.97893300 & 3.03919700 & -0.97473800 & $\mathrm{C}$ & -3.79257600 & 3.33302700 & -1.74890000 \\
\hline $\mathrm{F}$ & -2.52127400 & 5.19842900 & -0.84608100 & $\mathrm{C}$ & -3.28748400 & 4.26250900 & -0.93696100 \\
\hline $\mathrm{F}$ & -5.02392400 & 5.05099800 & 0.23054400 & $\mathrm{C}$ & -3.04800500 & 3.58614200 & 0.39960500 \\
\hline $\mathrm{F}$ & -5.94123200 & 2.67115400 & 1.17876100 & $\mathrm{C}$ & -4.40571700 & 3.31545100 & 1.10988400 \\
\hline \multirow[t]{2}{*}{$\mathrm{F}$} & -4.42232000 & 0.48704100 & 1.04882600 & $\mathrm{C}$ & -5.30808100 & 0.86676600 & 0.59868100 \\
\hline & & & & $\mathrm{N}$ & -5.24829200 & 0.17165300 & -0.68905500 \\
\hline 8 & & & & $\mathrm{C}$ & -4.12587700 & 0.67703300 & -1.49007300 \\
\hline $\mathrm{N}$ & 0.99347400 & -3.42217400 & -1.61687600 & $\mathrm{O}$ & -2.73498300 & 2.22095300 & -0.03285300 \\
\hline $\mathrm{C}$ & 0.65190500 & -2.07195300 & -2.05532600 & $\mathrm{C}$ & -4.24871900 & -2.30119300 & -0.10415700 \\
\hline $\mathrm{C}$ & -0.79035300 & -1.97378300 & -1.76849600 & $\mathrm{C}$ & -4.13979400 & -2.41598700 & 1.28184100 \\
\hline $\mathrm{C}$ & -1.27687800 & -3.22379400 & -1.12787000 & $\mathrm{C}$ & -2.98842600 & -2.98011800 & 1.81552400 \\
\hline $\mathrm{C}$ & 0.00305300 & -3.93345900 & -0.65835600 & $\mathrm{C}$ & -1.94652300 & -3.42317000 & 0.98996100 \\
\hline $\mathrm{C}$ & -1.62078200 & -0.87591900 & -2.00573600 & $\mathrm{C}$ & -2.10444300 & -3.33954100 & -0.39709900 \\
\hline $\mathrm{C}$ & -2.86829700 & -0.91115200 & -1.44655100 & $\mathrm{C}$ & -3.24802600 & -2.77217000 & -0.95100400 \\
\hline $\mathrm{C}$ & -3.45560400 & -2.08317200 & -0.72025300 & $\mathrm{~S}$ & -5.63905400 & -1.44758000 & -0.79552200 \\
\hline $\mathrm{C}$ & -2.37748700 & -2.97596800 & -0.08725300 & $\mathrm{O}$ & -5.68134300 & -1.73641400 & -2.21733800 \\
\hline $\mathrm{C}$ & -2.96474300 & -4.27310800 & 0.45123500 & $\mathrm{O}$ & -6.78078400 & -1.62272000 & 0.08317900 \\
\hline $\mathrm{C}$ & 3.13434300 & -2.81874000 & 0.04544100 & $\mathrm{C}$ & -5.23534100 & 4.56761000 & 1.35946000 \\
\hline $\mathrm{C}$ & 3.70572500 & -1.56183000 & -0.14850600 & $\mathrm{H}$ & -5.93071700 & 2.69039000 & -0.35553500 \\
\hline $\mathrm{C}$ & 4.16436200 & -0.85379900 & 0.95818000 & $\mathrm{H}$ & -4.17820300 & 2.82295900 & 2.06181900 \\
\hline $\mathrm{C}$ & 4.04612400 & -1.37659300 & 2.24858400 & $\mathrm{H}$ & -6.29017400 & 0.71967900 & 1.04872600 \\
\hline $\mathrm{C}$ & 3.45886600 & -2.63654100 & 2.41456000 & $\mathrm{H}$ & -4.52591600 & 0.52084200 & 1.29530800 \\
\hline $\mathrm{C}$ & 3.00545200 & -3.36610200 & 1.32231900 & $\mathrm{H}$ & -4.95611600 & -2.08807400 & 1.91895100 \\
\hline $\mathrm{C}$ & 4.56238200 & -0.60289900 & 3.43368900 & $\mathrm{H}$ & -2.88847800 & -3.07643000 & 2.89406200 \\
\hline $\mathrm{S}$ & 2.61326800 & -3.76393400 & -1.36461000 & $\mathrm{H}$ & -1.31567100 & -3.70086800 & -1.04916400 \\
\hline $\mathrm{O}$ & 3.28444600 & -3.22755700 & -2.53439500 & $\mathrm{H}$ & -3.37911400 & -2.70406700 & -2.02701500 \\
\hline $\mathrm{O}$ & 2.63934500 & -5.16858300 & -1.00202200 & $\mathrm{H}$ & -6.16692600 & 4.31075500 & 1.87059800 \\
\hline $\mathrm{O}$ & -4.34656500 & -1.68406900 & 0.27156000 & $\mathrm{H}$ & -4.69534700 & 5.28331300 & 1.98711900 \\
\hline $\mathrm{Si}$ & -5.84665200 & -0.91185900 & 0.06306800 & $\mathrm{H}$ & -5.49438700 & 5.05971100 & 0.41678600 \\
\hline $\mathrm{C}$ & -5.61245900 & 0.95636900 & -0.11072900 & $\mathrm{H}$ & -3.22249600 & 0.05933600 & -1.38884900 \\
\hline $\mathrm{C}$ & -5.75367900 & 1.54656000 & -1.52192700 & $\mathrm{H}$ & -4.40063500 & 0.70344200 & -2.54767000 \\
\hline $\mathrm{C}$ & -6.75695200 & -1.32159800 & 1.64539200 & $\mathrm{H}$ & -4.16458000 & 3.44017500 & -2.76041300 \\
\hline $\mathrm{C}$ & -5.97428800 & -0.89378100 & 2.89571400 & $\mathrm{H}$ & -3.13731400 & 5.31698800 & -1.13087300 \\
\hline
\end{tabular}




\begin{tabular}{|c|c|c|c|c|c|c|c|}
\hline $\mathrm{C}$ & -6.68279800 & -1.64860200 & -1.45551200 & $\mathrm{H}$ & -2.25237400 & 3.97780500 & 1.02908300 \\
\hline $\mathrm{C}$ & -8.18901800 & -1.33969400 & -1.47023500 & $\mathrm{Si}$ & -0.90356700 & 1.43022200 & -0.22264900 \\
\hline $\mathrm{H}$ & 0.89981100 & -1.89250600 & -3.10894400 & B & 2.23175300 & -0.12312600 & -0.04840600 \\
\hline $\mathrm{H}$ & 1.14086500 & -1.26517900 & -1.47179700 & $\mathrm{C}$ & 3.17648300 & 1.19760500 & -0.05049100 \\
\hline $\mathrm{H}$ & -1.70973100 & -3.80251100 & -1.96912200 & $\mathrm{C}$ & 4.12253100 & 1.53757800 & 0.90895400 \\
\hline $\mathrm{H}$ & -0.05604100 & -5.01867500 & -0.74112900 & 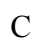 & 2.99192700 & 2.15369100 & -1.04415800 \\
\hline $\mathrm{H}$ & 0.23040500 & -3.65483600 & 0.38003400 & $\mathrm{C}$ & 4.81632900 & 2.74539800 & 0.90283100 \\
\hline $\mathrm{H}$ & -1.24245300 & 0.01022100 & -2.49976700 & $\mathrm{C}$ & 3.64552200 & 3.37344600 & -1.08787200 \\
\hline $\mathrm{H}$ & -3.97569000 & -2.67646200 & -1.50645500 & $\mathrm{C}$ & 4.57260100 & 3.67279800 & -0.09762600 \\
\hline $\mathrm{H}$ & -1.93410400 & -2.40236600 & 0.73484400 & $\mathrm{C}$ & 2.17109500 & -0.86622500 & 1.40538800 \\
\hline $\mathrm{H}$ & -3.75059700 & -4.05297100 & 1.17692800 & . & 1.62331300 & -0.19370100 & 2.49124500 \\
\hline $\mathrm{H}$ & -2.19565900 & -4.86897300 & 0.95140700 & $\mathrm{C}$ & 2.60209700 & -2.156 & 6900 \\
\hline $\mathrm{H}$ & -3.39499200 & -4.87592800 & -0.35739700 & $\mathrm{C}$ & 1.45376300 & -0.73318800 & 3.7567190 \\
\hline $\mathrm{H}$ & 3.81969600 & -1.16477700 & -1.15416500 & $\mathrm{C}$ & 2.46926700 & -2.74223700 & 2.95769900 \\
\hline $\mathrm{H}$ & 4.62708600 & 0.11857500 & 0.822 & $\mathrm{C}$ & 1.88626000 & -2.03001500 & .99468000 \\
\hline $\mathrm{H}$ & 3.34648400 & -3.04610800 & 3.41547800 & 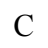 & 2.49071300 & -1.15702000 & -1.27437000 \\
\hline $\mathrm{H}$ & 2.58383100 & -4.35866900 & 1.44707300 & $\mathrm{C}$ & 1.60861200 & -2.22105500 & -1.42670400 \\
\hline $\mathrm{H}$ & 3.98137300 & -0.81935100 & 4.33472900 & $\mathrm{C}$ & 3.47098100 & -1.07897000 & -2.25504300 \\
\hline $\mathrm{H}$ & 5.60625500 & -0.86562600 & 3.63888700 & $\mathrm{C}$ & 1.66313600 & -3.14868200 & -2.44937800 \\
\hline $\mathrm{H}$ & 4.52010700 & 0.47383200 & 3.24606100 & $\mathrm{C}$ & 3.57013600 & -1.98805700 & -3.30755700 \\
\hline $\mathrm{H}$ & -4.63436100 & 1.22985900 & 0.30501800 & $\mathrm{C}$ & 2.66254200 & -3.02973800 & -3.40754900 \\
\hline $\mathrm{H}$ & -5.04748500 & 1.10810400 & -2.23746900 & $\mathrm{~F}$ & 4.39185400 & -0.10678200 & -2.23887400 \\
\hline $\mathrm{H}$ & -5.56735400 & 2.62301100 & -1.51300800 & $\mathrm{~F}$ & 4.53210400 & -1.86133600 & -4.22143100 \\
\hline $\mathrm{H}$ & -6.75474700 & 1.37827900 & -1.93143200 & $\mathrm{~F}$ & 2.74211400 & -3.90358500 & -4.40821800 \\
\hline $\mathrm{H}$ & -6.95424400 & -2.40100900 & 1.67314300 & $\mathrm{~F}$ & 0.76392900 & -4.13719700 & -2.52832600 \\
\hline $\mathrm{H}$ & -5.00166300 & -1.39273800 & 2.93797800 & $\mathrm{~F}$ & 0.60252900 & -2.36435200 & -0.53691400 \\
\hline $\mathrm{H}$ & -6.51859100 & -1.13834700 & 3.81245400 & $\Gamma$ & 2.09094800 & 1.91845900 & -2.02178400 \\
\hline $\mathrm{H}$ & -5.79062200 & 0.18595700 & 2.89984100 & $\mathrm{~F}$ & 3.37761400 & 4.26074600 & -2.04988100 \\
\hline $\mathrm{H}$ & -6.53033900 & -2.73625000 & -1.46341100 & $\Gamma$ & 4.41425200 & 0.70203600 & 1.91498900 \\
\hline $\mathrm{H}$ & -8.69416600 & -1.81771600 & -0.62520200 & $\mathrm{~F}$ & 5.70742900 & 3.02050600 & 1.85492300 \\
\hline $\mathrm{H}$ & -8.66590400 & -1.69732800 & -2.38718700 & $\mathrm{~F}$ & 5.21329200 & 4.83976700 & -0.1 \\
\hline $\mathrm{H}$ & -8.38143600 & -0.26320900 & -1.39856200 & 1 & 1.21243900 & 1.08303500 & 2.33440300 \\
\hline $\mathrm{H}$ & -3.49502000 & -0.02385600 & -1.51753300 & $\mathrm{~F}$ & 0.88756800 & -0.01842800 & 4.73274500 \\
\hline $\mathrm{B}$ & 0.62226100 & 1.35077700 & -0.20482900 & $\mathrm{~F}$ & 1.74284700 & -2.58032500 & 5.19836700 \\
\hline $\mathrm{C}$ & 1.98304900 & 1.40555600 & -1.11384800 & $\Gamma$ & 2.88283900 & -3.99219200 & 3.16605800 \\
\hline $\mathrm{C}$ & 1.95296300 & 1.02050500 & -2.45123800 & $\mathrm{C}$ & -1.36114200 & -0.00085500 & 0.90155600 \\
\hline $\mathrm{C}$ & 3.25313400 & 1.75116700 & -0.66064900 & $\mathrm{H}$ & -2.18683200 & -0.54533000 & 0.42082800 \\
\hline $\mathrm{C}$ & 3.06864000 & 0.92926200 & -3.27511800 & $\Pi$ & -0.52697600 & -0.70917200 & 0.93703500 \\
\hline $\mathrm{C}$ & 4.40131600 & 1.67567700 & -1.43918400 & $\mathrm{C}$ & -0.92185600 & 1.24039400 & -2.09355900 \\
\hline $\mathrm{C}$ & 4.31339300 & 1.26142600 & -2.76142300 & $\mathrm{H}$ & -1.87957100 & 1.62107400 & -2.46743000 \\
\hline $\mathrm{C}$ & 0.87780100 & 0.75602800 & 1.29774700 & $\mathrm{H}$ & -0.16504400 & 1.92549500 & -2.48740900 \\
\hline $\mathrm{C}$ & 1.34124600 & 1.47783800 & 2.39636700 & $\mathrm{C}$ & 0.05880000 & 2.89641000 & 0.43702600 \\
\hline $\mathrm{C}$ & 0.60342900 & -0.57363600 & 1.57727800 & $\mathrm{C}$ & -0.65241600 & -0.17545600 & -2.62140800 \\
\hline $\mathrm{C}$ & 1.56185000 & 0.90989200 & 3.64719500 & $\mathrm{H}$ & -1.00889900 & -0.28327700 & -3.64994200 \\
\hline
\end{tabular}




$$
\begin{array}{|lrrr}
\text { C } & 0.77057700 & -1.18319500 & 2.80982500 \\
\text { C } & 1.27761600 & -0.43307000 & 3.85579700 \\
\text { C } & -0.21175900 & 2.74731100 & -0.17135000 \\
\text { C } & -1.60055700 & 2.73783700 & -0.20444200 \\
\text { C } & 0.35806000 & 4.01470900 & -0.10444700 \\
\text { C } & -2.38778000 & 3.87968200 & -0.17674600 \\
\text { C } & -0.38638300 & 5.18914200 & -0.08069000 \\
\text { C } & -1.77246300 & 5.12071500 & -0.11636200 \\
\text { H } & -0.09037100 & 0.54994900 & -0.74834000 \\
\text { F } & 1.59533300 & 2.78443200 & 2.28590500 \\
\text { F } & 2.06655100 & 1.63240800 & 4.64737800 \\
\text { F } & 1.53892300 & -1.00484100 & 5.03297400 \\
\text { F } & 0.49636500 & -2.48442300 & 2.96785900 \\
\text { F } & 0.12457200 & -1.39815700 & 0.60656000 \\
\text { F } & 0.78297700 & 0.66721000 & -3.04038100 \\
\text { F } & 2.94644900 & 0.53963800 & -4.54413000 \\
\text { F } & 5.40030300 & 1.19234900 & -3.52311900 \\
\text { F } & 5.59065200 & 1.96824900 & -0.91013500 \\
\text { F } & 3.44369600 & 2.13657400 & 0.60782700 \\
\text { F } & 1.68791100 & 4.14998300 & -0.06186400 \\
\text { F } & 0.21217600 & 6.37849200 & -0.02493600 \\
\text { F } & -2.50475800 & 6.23292200 & -0.09279000 \\
\text { F } & -3.72421200 & 3.79254200 & -0.20966200 \\
\text { F } & -2.27759700 & 1.56377500 & -0.26787900 \\
\text { H } & -7.73818500 & -0.82908900 & 1.62751400 \\
\text { H } & -6.34935000 & 1.43692100 & 0.54705000 \\
\text { H } & -6.21749700 & -1.26236600 & -2.37289600 \\
& & &
\end{array}
$$

\section{TS4}

$\begin{array}{llll}\text { C } & 0.64339900 & -1.14060700 & 3.36592200\end{array}$

$\begin{array}{llll}\text { C } & 0.12416100 & -1.64269300 & 1.97560600\end{array}$

$\begin{array}{llll}\text { C } & -1.09144200 & -2.50039100 & 2.25979500\end{array}$

$\begin{array}{llll}\text { C } & -2.04282600 & -1.65306400 & 2.65391000\end{array}$

$\begin{array}{llll}\text { C } & -1.42417200 & -0.26693900 & 2.57777800\end{array}$

$\begin{array}{llll}\text { C } & -0.39909400 & -0.07319900 & 3.73553600\end{array}$

$\begin{array}{llll}\mathrm{C} & 2.08332300 & -0.73750900 & 3.06736900\end{array}$

$\begin{array}{llll}\mathrm{N} & 2.46732500 & -1.80454900 & 2.13031900\end{array}$

$\begin{array}{llll}\text { C } & 1.36056600 & -2.05970100 & 1.19224400\end{array}$

$\begin{array}{llll}\text { O } & -0.51102900 & -0.42671500 & 1.46342400\end{array}$

$\begin{array}{llll}\text { C } & 4.08066800 & -0.35681300 & 0.46220500\end{array}$

$\begin{array}{llll}\text { C } & 4.46482800 & 0.85893500 & 1.02356600\end{array}$

$\begin{array}{llll}\text { C } & 4.38250900 & 2.01122700 & 0.25305700\end{array}$

$\begin{array}{llll}\text { C } & 3.91155100 & 1.96658500 & -1.06274200\end{array}$

$\begin{array}{llll}\text { C } & 3.57395900 & 0.72883400 & -1.61573300\end{array}$

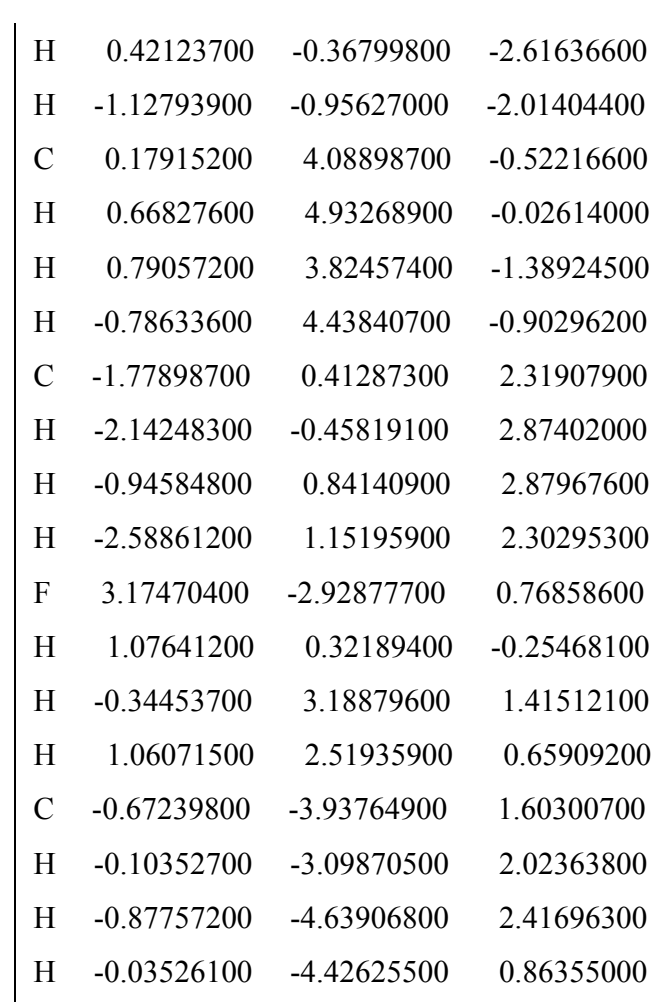

\section{TS3}

$\begin{array}{llll}\mathrm{N} & -3.10496600 & -0.50359400 & -1.49237900\end{array}$

$\begin{array}{llll}\text { C } & -1.73046800 & -0.01941300 & -1.57356400\end{array}$

$\begin{array}{llll}\text { C } & -1.90160200 & 1.41018000 & -1.82467300\end{array}$

C $\quad-3.16349900 \quad 1.62824900 \quad-2.60786900$

$\begin{array}{llll}\text { C } & -3.98623800 & 0.34789000 & -2.33904400\end{array}$

$\begin{array}{llll}\text { C } & -0.96030500 & 2.49339300 & -1.66682200\end{array}$

$\begin{array}{llll}\text { C } & -1.56236700 & 3.60080700 & -1.20047800\end{array}$

$\begin{array}{llll}\text { C } & -3.03610100 & 3.37323200 & -0.91152200\end{array}$

C $\quad-3.77382800 \quad 3.00099700 \quad-2.21313500$

$\begin{array}{llll}\text { O } & -3.10319300 & 2.14831600 & -0.17714100\end{array}$

C $\quad-3.68863000 \quad 4.05959400 \quad-3.30566500$

$\begin{array}{llll}\text { C } & -2.33329500 & -2.84344600 & -0.46604900\end{array}$

$\begin{array}{llll}\text { C } & -1.01054100 & -3.22356800 & -0.67236700\end{array}$

C $\quad-0.28636500 \quad-3.72510200 \quad 0.40374100$

$\begin{array}{llll}\text { C } & -0.86253400 & -3.82845900 & 1.67293300\end{array}$

$\begin{array}{llll}\text { C } & -2.19586100 & -3.43759500 & 1.84628800\end{array}$

$\begin{array}{llll}\text { C } & -2.94343600 & -2.95316600 & 0.78143500\end{array}$

C $\quad-0.05639600 \quad-4.36092600 \quad 2.82825400$

S $\quad-3.25837300 \quad-2.16160100 \quad-1.80883300$

$\begin{array}{llll}\mathrm{O} & -2.56936600 & -2.46515700 & -3.05866400\end{array}$

$\begin{array}{llll}\mathrm{O} & -4.67828600 & -2.43204700 & -1.66009000\end{array}$

$\mathrm{H} \quad-1.19450800 \quad-0.39429100 \quad-2.47266800$

$\mathrm{H} \quad-1.12755300 \quad-0.25771900 \quad-0.69489000$

$\begin{array}{llll}\mathrm{H} & -2.83780800 & 1.65950700 & -3.65868200\end{array}$ 


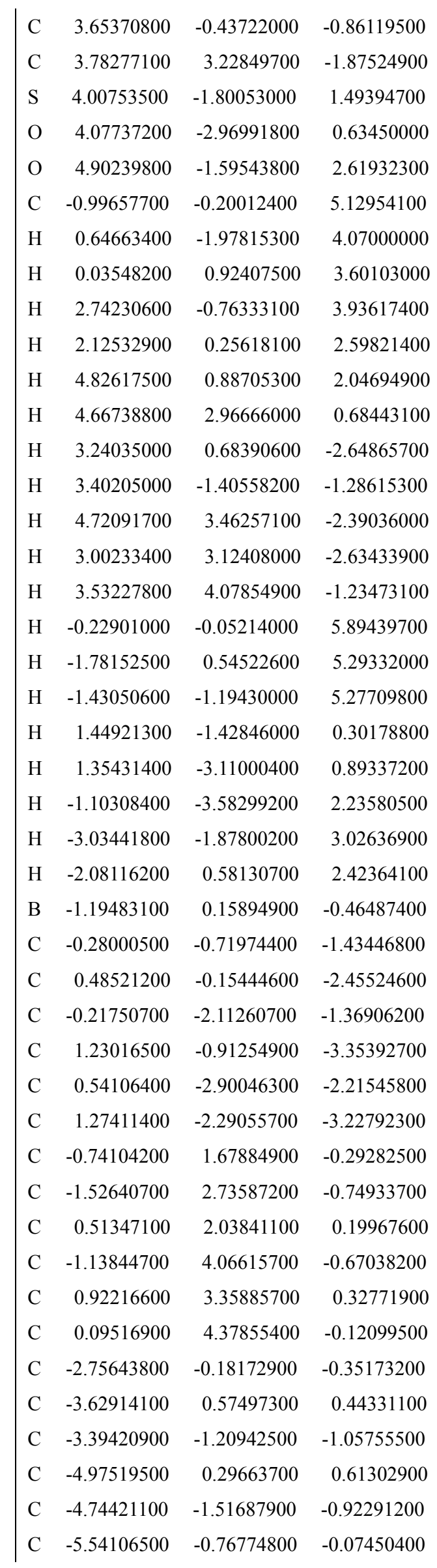

\begin{tabular}{|c|c|c|c|}
\hline & -4.24769700 & -0.15298300 & -3.27706500 \\
\hline & -4.90350600 & 0.56159900 & 1786007 \\
\hline & 0.10697000 & 34143100 & 1.77718300 \\
\hline & -1.02555400 & 4.51043100 & 26900 \\
\hline & -3.51459800 & 4.20032800 & -0.37941400 \\
\hline & -4.81884800 & 2.82873000 & 100 \\
\hline & -4.28496400 & 3.76482800 & 334900 \\
\hline & -4.07 & 0 & 00 \\
\hline & -2.65665800 & 3400 & 900 \\
\hline & -0.56 & 00 & 00 \\
\hline & 0.74558500 & 6500 & 6800 \\
\hline & -2.64792900 & -3.51002600 & 6200 \\
\hline & -3.98295100 & 00 & 000 \\
\hline t & 1.00640200 & 1000 & 1400 \\
\hline & -0.38446900 & -3.91967500 & 8300 \\
\hline & -0.16648800 & 0 & 00 \\
\hline & -2.92639900 & 2.04833300 & 5900 \\
\hline C & -3.56546400 & 0.35859800 & 400 \\
\hline & -2.85664000 & 0.40955500 & 00 \\
\hline C & -4.06397100 & 3.39919200 & 700 \\
\hline & -4.9 & 0 & 0 \\
\hline C & -1.12586300 & 2.334 & 1900 \\
\hline & -0.83 & 0 & 00 \\
\hline c & -0.81 & 200 & 00 \\
\hline & 0.26382400 & 9100 & 1400 \\
\hline $\mathrm{H}$ & -1.266 & 0 & 00 \\
\hline & -1.19 & 1.08087900 & 00 \\
\hline C & -4.97277600 & 0.05557300 & 56000 \\
\hline $\mathrm{H}$ & -4.95231900 & 00557200 & 10 \\
\hline & -5.37995000 & -0.85583800 & 9100 \\
\hline & -5.6 & $0.80540 / 00$ & 84800 \\
\hline $\mathrm{C}$ & 00 & 0 & 00 \\
\hline & -5.14153500 & 3.95130600 & .03776700 \\
\hline H & - & & 3.73291600 \\
\hline H & -3.66066100 & 3.041 & 7800 \\
\hline $\mathrm{H}$ & -3.55567100 & 0.31892000 & 01500 \\
\hline $\mathrm{H}$ & -0.51558700 & 68423100 & 18100 \\
\hline $\mathrm{H}$ & -3.55648500 & 4.37122700 & 400 \\
\hline $\mathrm{B}$ & 1.6 & 00 & 0 \\
\hline $\mathrm{C}$ & 1. & 00 & 00 \\
\hline C & 1.35731700 & -0.75375500 & -2.45960400 \\
\hline C & 2.28 & -2. & -0.9 \\
\hline C & 0 & -1 & -3 \\
\hline $\mathrm{C}$ & 2.29353200 & -3.24708700 & -1.88159400 \\
\hline
\end{tabular}




\begin{tabular}{|c|c|c|c|c|c|c|c|}
\hline $\mathrm{F}$ & 1.39608200 & 1.10181300 & 0.56596000 & $\mathrm{C}$ & 1.82246600 & -2.99096400 & -3.16214400 \\
\hline $\mathrm{F}$ & 2.10664600 & 3.66695900 & 0.85739000 & $\mathrm{C}$ & 1.61816900 & -0.31025600 & 1.47936600 \\
\hline $\mathrm{F}$ & 0.48575400 & 5.64289500 & -0.02042200 & $\mathrm{C}$ & 2.68874100 & -0.40288700 & 2.36559800 \\
\hline $\mathrm{F}$ & -1.93232900 & 5.03473100 & -1.11796200 & $\mathrm{C}$ & 0.40543500 & -0.72852600 & 2.00964200 \\
\hline $\mathrm{F}$ & -2.71560700 & 2.50071700 & -1.31484000 & $\mathrm{C}$ & 2.56408200 & -0.84291600 & 3.67942800 \\
\hline $\mathrm{F}$ & -0.89873200 & -2.75703300 & -0.41325100 & $\mathrm{C}$ & 0.22340600 & -1.15110900 & 3.31764500 \\
\hline $\mathrm{F}$ & 0.57584600 & -4.22024000 & -2.06575200 & $\mathrm{C}$ & 1.31824000 & -1.21787800 & 4.16307100 \\
\hline $\mathrm{F}$ & 1.99686300 & -3.01878600 & -4.06472900 & $\mathrm{C}$ & 2.84493900 & 1.36351000 & -0.29495800 \\
\hline $\mathrm{F}$ & 1.93657400 & -0.30877500 & -4.31014100 & $\mathrm{C}$ & 4.16380000 & 1.04055800 & -0.60330400 \\
\hline $\mathrm{F}$ & 0.53030100 & 1.16706100 & -2.65285900 & $\mathrm{C}$ & 2.58190400 & 2.72568300 & -0.22860900 \\
\hline $\mathrm{F}$ & -2.74974200 & -1.95681400 & -1.95541100 & $\mathrm{C}$ & 5.15100400 & 1.98702900 & -0.84255900 \\
\hline $\mathrm{F}$ & -5.27306100 & -2.51692700 & -1.62060100 & $\mathrm{C}$ & 3.53407700 & 3.71127500 & -0.45990700 \\
\hline $\mathrm{F}$ & -5.72678700 & 1.05104100 & 1.41006000 & $\mathrm{C}$ & 4.83183100 & 3.33663700 & -0.76985900 \\
\hline $\mathrm{F}$ & -3.19300400 & 1.65526600 & 1.11150600 & $\mathrm{H}$ & 0.62449100 & 0.79685500 & -0.25912800 \\
\hline \multirow[t]{2}{*}{$\mathrm{F}$} & -6.82800800 & -1.04883900 & 0.06533900 & $\mathrm{~F}$ & 3.92440200 & -0.07203200 & 1.97509000 \\
\hline & & & & $\mathrm{F}$ & 3.62783500 & -0.91036100 & 4.47940600 \\
\hline \multicolumn{2}{|c|}{ TS5 } & & & $\mathrm{F}$ & 1.17154500 & -1.63637700 & 5.41953000 \\
\hline $\mathrm{C}$ & 1.13061900 & 1.92941100 & 2.66327500 & $\mathrm{~F}$ & -0.99671900 & -1.47049600 & 3.77388100 \\
\hline $\mathrm{C}$ & 0.86286400 & 0.51675700 & 3.08528100 & $\mathrm{~F}$ & -0.70996400 & -0.71813500 & 1.24137100 \\
\hline $\mathrm{C}$ & -0.22019000 & 0.28322000 & 3.99177700 & $\mathrm{~F}$ & 0.86756200 & 0.45954800 & -2.82207900 \\
\hline $\mathrm{C}$ & -1.35276900 & 0.91501900 & 3.61321400 & $\mathrm{~F}$ & 0.88085100 & -1.43783800 & -4.67328100 \\
\hline $\mathrm{C}$ & -1.23882900 & 1.51580700 & 2.22170200 & $\mathrm{~F}$ & 1.83432400 & -3.94187300 & -4.09114400 \\
\hline $\mathrm{C}$ & -0.17440400 & 2.63230100 & 2.22866900 & $\mathrm{~F}$ & 2.72943300 & -4.46780900 & -1.56474400 \\
\hline $\mathrm{C}$ & 2.31378500 & 1.78628800 & 1.70054400 & $\mathrm{~F}$ & 2.74880200 & -2.58753300 & 0.28493300 \\
\hline $\mathrm{N}$ & 3.01807000 & 0.65987900 & 2.32505800 & $\mathrm{~F}$ & 1.33834500 & 3.17281600 & 0.06469000 \\
\hline $\mathrm{C}$ & 2.02654100 & -0.33580700 & 2.73335900 & $\mathrm{~F}$ & 3.21002700 & 5.00600600 & -0.38431100 \\
\hline $\mathrm{O}$ & -0.67202100 & 0.46268100 & 1.46884900 & $\mathrm{~F}$ & 5.76365300 & 4.26161500 & -0.99446200 \\
\hline $\mathrm{C}$ & 4.15535500 & -0.32140600 & 0.01377000 & $\mathrm{~F}$ & 6.39846800 & 1.61862300 & -1.13402000 \\
\hline $\mathrm{C}$ & 4.29894400 & 0.64255500 & -0.98235400 & $\mathrm{~F}$ & 4.53546600 & -0.24369300 & -0.67165000 \\
\hline $\mathrm{C}$ & 3.99278000 & 0.30194400 & -2.29321200 & & & & \\
\hline $\mathrm{C}$ & 3.54559700 & -0.98155500 & -2.61783100 & 3a & & & \\
\hline $\mathrm{C}$ & 3.45725700 & -1.94248700 & -1.60428400 & $\mathrm{~N}$ & -2.29866900 & -1.65869800 & -0.03000900 \\
\hline $\mathrm{C}$ & 3.74438800 & -1.62028900 & -0.28296500 & $\mathrm{C}$ & -1.90374100 & -1.42087200 & 1.37114700 \\
\hline $\mathrm{C}$ & 3.11098300 & -1.30935200 & -4.02017200 & $\mathrm{C}$ & -0.49284500 & -0.89605000 & 1.22377500 \\
\hline S & 4.47892400 & 0.13136300 & 1.69436300 & $\mathrm{C}$ & -0.07802600 & -1.00889700 & -0.22654100 \\
\hline $\mathrm{O}$ & 4.78307800 & -1.06698200 & 2.45710900 & $\mathrm{C}$ & -1.42633900 & -0.91069400 & -0.94625300 \\
\hline $\mathrm{O}$ & 5.33355900 & 1.30261100 & 1.68666400 & $\mathrm{C}$ & 0.24350600 & -0.29091900 & 2.15085900 \\
\hline $\mathrm{C}$ & -0.54100400 & 3.84070800 & 3.08296000 & $\mathrm{C}$ & 1.59395700 & 0.29957800 & 1.84268600 \\
\hline $\mathrm{H}$ & 1.52000700 & 2.38589300 & 3.58955900 & $\mathrm{C}$ & 2.09854300 & -0.04869600 & 0.43951600 \\
\hline $\mathrm{H}$ & -0.04819900 & 2.93294900 & 1.18153400 & $\mathrm{C}$ & 0.97546800 & 0.02896900 & -0.60241100 \\
\hline $\mathrm{H}$ & 2.96328700 & 2.66252000 & 1.67593900 & $\mathrm{C}$ & 1.50230000 & -0.17253400 & -2.01937700 \\
\hline $\mathrm{H}$ & 1.96841200 & 1.53948900 & 0.68751400 & $\mathrm{C}$ & -4.58318000 & -0.15103500 & -0.18114400 \\
\hline $\mathrm{H}$ & 4.65495700 & 1.63581200 & -0.72670500 & $\mathrm{C}$ & -5.10265300 & 0.23293500 & 1.05153000 \\
\hline $\mathrm{H}$ & 4.08044400 & 1.05125000 & -3.07441200 & $\mathrm{C}$ & -5.57272500 & 1.53317600 & 1.21134000 \\
\hline
\end{tabular}




\begin{tabular}{|c|c|c|c|}
\hline $\mathrm{H}$ & 3.16450000 & -2.95822800 & -1.85734000 \\
\hline $\mathrm{H}$ & 3.69462000 & -2.36840500 & 0.50355400 \\
\hline $\mathrm{H}$ & 2.03539900 & -1.12492700 & -4.11956600 \\
\hline $\mathrm{H}$ & 3.63129400 & -0.68943200 & -4.75444600 \\
\hline $\mathrm{H}$ & 3.28466500 & -2.36150600 & -4.25873800 \\
\hline $\mathrm{H}$ & 0.23439600 & 4.60955600 & 3.02275200 \\
\hline $\mathrm{H}$ & -1.48062600 & 4.28427300 & 2.74053000 \\
\hline $\mathrm{H}$ & -0.65732800 & 3.56316500 & 4.13650100 \\
\hline $\mathrm{H}$ & 1.70019900 & -0.99842300 & 1.91013900 \\
\hline $\mathrm{H}$ & 2.38408600 & -0.96212600 & 3.55518300 \\
\hline $\mathrm{H}$ & -0.13965600 & -0.41990100 & 4.81361700 \\
\hline $\mathrm{H}$ & -2.28331400 & 0.86182200 & 4.16692100 \\
\hline $\mathrm{H}$ & -2.19811800 & 1.85526300 & 1.81707300 \\
\hline B & -1.19553200 & 0.06811500 & 0.11297800 \\
\hline $\mathrm{C}$ & -0.41213900 & -1.31747800 & -0.27603000 \\
\hline $\mathrm{C}$ & -0.09958300 & -2.27713200 & 0.68407500 \\
\hline $\mathrm{C}$ & -0.11484600 & -1.69006700 & -1.58568800 \\
\hline $\mathrm{C}$ & 0.51989200 & -3.48960700 & 0.39510200 \\
\hline $\mathrm{C}$ & 0.47835600 & -2.89802900 & -1.91882000 \\
\hline $\mathrm{C}$ & 0.80746300 & -3.80288400 & -0.92148000 \\
\hline $\mathrm{C}$ & -0.87864800 & 1.35331300 & -0.86457900 \\
\hline $\mathrm{C}$ & -1.75254300 & 2.41955200 & -1.05047300 \\
\hline $\mathrm{C}$ & 0.38956300 & 1.55733700 & -1.39529300 \\
\hline $\mathrm{C}$ & -1.41314700 & 3.58913400 & -1.72309400 \\
\hline $\mathrm{C}$ & 0.78266100 & 2.70574800 & -2.06724400 \\
\hline $\mathrm{C}$ & -0.13332000 & 3.73420700 & -2.23849900 \\
\hline $\mathrm{C}$ & -2.78856100 & -0.33799300 & 0.14570800 \\
\hline $\mathrm{C}$ & -3.46443900 & -0.80719400 & 1.26452800 \\
\hline $\mathrm{C}$ & -3.53251800 & -0.37165700 & -1.03277100 \\
\hline $\mathrm{C}$ & -4.78541600 & -1.24278200 & 1.24582600 \\
\hline $\mathrm{C}$ & -4.84894400 & -0.80261600 & -1.10371600 \\
\hline $\mathrm{C}$ & -5.48410500 & -1.24225400 & 0.05015700 \\
\hline F & 1.34368200 & 0.62297500 & -1.23054600 \\
\hline F & 2.02824900 & 2.83133700 & -2.53212700 \\
\hline F & 0.21451100 & 4.84971300 & -2.87298200 \\
\hline F & -2.29844500 & 4.57489500 & -1.86083100 \\
\hline F & -2.99533300 & 2.38424400 & -0.53791400 \\
\hline F & -0.36484900 & -0.85894200 & -2.60644100 \\
\hline F & 0.81098300 & -3.17381300 & -3.18182600 \\
\hline F & 1.45060500 & -4.92746800 & -1.23126000 \\
\hline $\mathrm{F}$ & 0.83747700 & -4.33871500 & 1.37282900 \\
\hline F & -0.36810400 & -2.07542000 & 1.99039500 \\
\hline $\mathrm{F}$ & -2.97361600 & 0.02396200 & -2.18476300 \\
\hline $\mathrm{F}$ & -5.50474800 & -0.80504800 & -2.26270700 \\
\hline
\end{tabular}

\begin{tabular}{|c|c|c|c|}
\hline $\mathrm{C}$ & -5.53150300 & 2.44851400 & 0.15783500 \\
\hline $\mathrm{C}$ & -5.01133200 & 2.03396100 & -1.07360100 \\
\hline $\mathrm{C}$ & -4.53822900 & 0.74056200 & -1.25222200 \\
\hline $\mathrm{C}$ & -6.06115600 & 3.84923500 & 0.32527300 \\
\hline S & -3.91006700 & -1.78726100 & -0.37906300 \\
\hline $\mathrm{O}$ & -4.48199500 & -2.62153900 & 0.66461400 \\
\hline $\mathrm{O}$ & -3.99542600 & -2.12457400 & -1.78963700 \\
\hline $\mathrm{O}$ & 3.13599400 & 0.84544200 & 0.08795400 \\
\hline $\mathrm{Si}$ & 4.75422800 & 0.41817700 & 0.03794100 \\
\hline $\mathrm{C}$ & 4.98733500 & -1.00695500 & -1.17790100 \\
\hline $\mathrm{C}$ & 6.39405200 & -1.61958000 & -1.14602900 \\
\hline $\mathrm{C}$ & 5.34757900 & -0.05852600 & 1.76694200 \\
\hline $\mathrm{C}$ & 6.85661900 & 0.12939200 & 1.98502200 \\
\hline $\mathrm{C}$ & 5.62586100 & 1.96725500 & -0.56222500 \\
\hline $\mathrm{C}$ & 4.98126900 & 2.55937100 & -1.82290600 \\
\hline $\mathrm{H}$ & -1.97432000 & -2.35808700 & 1.92926100 \\
\hline $\mathrm{H}$ & -2.54834200 & -0.67581200 & 1.86102600 \\
\hline $\mathrm{H}$ & 0.32233600 & -2.01794600 & -0.41277800 \\
\hline $\mathrm{H}$ & -1.44827800 & -1.37667400 & -1.93241000 \\
\hline $\mathrm{H}$ & -1.73196900 & 0.14622500 & -1.03217000 \\
\hline $\mathrm{H}$ & -0.13643200 & -0.17719500 & 3.16506500 \\
\hline $\mathrm{H}$ & 2.33555300 & -0.01984800 & 2.58619300 \\
\hline $\mathrm{H}$ & 1.54362100 & 1.39482800 & 1.91356800 \\
\hline $\mathrm{H}$ & 2.46924700 & -1.08984500 & 0.44343100 \\
\hline $\mathrm{H}$ & 0.52473200 & 1.02952600 & -0.51879400 \\
\hline $\mathrm{H}$ & 2.25738500 & 0.57963900 & -2.26252600 \\
\hline $\mathrm{H}$ & 0.69443600 & -0.09939700 & -2.75494700 \\
\hline $\mathrm{H}$ & 1.96065000 & -1.16426400 & -2.12249000 \\
\hline $\mathrm{H}$ & -5.15922700 & -0.49148400 & 1.85821000 \\
\hline $\mathrm{H}$ & -5.98573400 & 1.84026600 & 2.16842200 \\
\hline $\mathrm{H}$ & -4.98552200 & 2.73441700 & -1.90448600 \\
\hline $\mathrm{H}$ & -4.16056500 & 0.40527600 & -2.21336700 \\
\hline $\mathrm{H}$ & -7.01379400 & 3.96892600 & -0.20183100 \\
\hline $\mathrm{H}$ & -6.22957500 & 4.08738500 & 1.37822800 \\
\hline $\mathrm{H}$ & -5.36435100 & 4.58558900 & -0.08576200 \\
\hline $\mathrm{H}$ & 4.24397100 & -1.78817800 & -0.97024300 \\
\hline $\mathrm{H}$ & 6.59532600 & -2.09039900 & -0.17805000 \\
\hline $\mathrm{H}$ & 6.51997000 & -2.38716500 & -1.91584300 \\
\hline $\mathrm{H}$ & 7.16923700 & -0.86223200 & -1.31020800 \\
\hline $\mathrm{H}$ & 4.79797700 & 0.56756600 & 2.48241400 \\
\hline $\mathrm{H}$ & 7.14826100 & 1.17251500 & 1.82340600 \\
\hline $\mathrm{H}$ & 7.15362000 & -0.14108800 & 3.00321300 \\
\hline $\mathrm{H}$ & 7.44803700 & -0.48362800 & 1.29761300 \\
\hline $\mathrm{H}$ & 5.61379600 & 2.70917200 & 0.24717100 \\
\hline
\end{tabular}




\begin{tabular}{|c|c|c|c|c|c|c|c|}
\hline $\mathrm{F}$ & -5.37518200 & -1.66548300 & 2.36508700 & $\mathrm{H}$ & 3.93113600 & 2.80560000 & -1.63979300 \\
\hline $\mathrm{F}$ & -2.85603000 & -0.86374900 & 2.46662500 & $\mathrm{H}$ & 5.49185800 & 3.47088200 & -2.14920000 \\
\hline \multirow[t]{2}{*}{$\mathrm{F}$} & -6.74557900 & -1.66108100 & 0.00542100 & $\mathrm{H}$ & 5.01326600 & 1.84969500 & -2.65723900 \\
\hline & & & & $\mathrm{H}$ & 6.68422600 & 1.73935500 & -0.74703300 \\
\hline \multicolumn{2}{|c|}{ TS6 } & & & $\mathrm{H}$ & 5.05899000 & -1.09574500 & 1.98687200 \\
\hline $\mathrm{N}$ & -4.85995800 & 1.90345800 & -0.38583400 & $\mathrm{H}$ & 4.75588000 & -0.63906100 & -2.18636300 \\
\hline $\mathrm{C}$ & -4.28809200 & 1.64568700 & 0.93896400 & & & & \\
\hline $\mathrm{C}$ & -2.83536000 & 1.45558900 & 0.63177400 & 9 & & & \\
\hline C & -2.62117100 & 1.49995000 & -0.84909300 & C & 0.77174200 & -1.18356300 & 3.27851400 \\
\hline $\mathrm{C}$ & -4.02583700 & 1.27236000 & -1.41582200 & $\mathrm{C}$ & 0.23341200 & -1.70406900 & 1.90536700 \\
\hline $\mathrm{C}$ & -1.80384500 & 1.28181900 & 1.49357600 & C & -0.95399200 & -2.58221400 & 2.23872400 \\
\hline $\mathrm{C}$ & -0.51548200 & 0.98824200 & 0.96396500 & $\mathrm{C}$ & -1.90709400 & -1.76293000 & 2.68336100 \\
\hline $\mathrm{C}$ & -1.50944800 & 0.56244900 & -1.31367500 & $\mathrm{C}$ & -1.34679300 & -0.35553600 & 2.58512500 \\
\hline $\mathrm{C}$ & -1.20141800 & 0.71037900 & -2.79838300 & C & -0.26163300 & -0.11598800 & 3.66701200 \\
\hline $\mathrm{C}$ & -6.91718700 & 0.09541400 & -0.35830600 & $\mathrm{C}$ & 2.22372900 & -0.80669700 & 3.00370100 \\
\hline $\mathrm{C}$ & -6.90797500 & -0.72375600 & -1.48724200 & $\mathrm{~N}$ & 2.58632300 & -1.84179600 & 2.02270000 \\
\hline $\mathrm{C}$ & -7.15540100 & -2.08160400 & -1.33384300 & C & 1.46463800 & -2.08512400 & 1.09957600 \\
\hline $\mathrm{C}$ & -7.41097300 & -2.63167000 & -0.07225300 & $\mathrm{O}$ & -0.48197300 & -0.48858300 & 1.40714100 \\
\hline 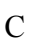 & -7.42842800 & -1.78476300 & 1.03906200 & C & 4.08906600 & -0.30621400 & 0.33711500 \\
\hline 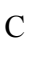 & -7.18376500 & -0.42148700 & 0.90716900 & C & 4.49654300 & 0.89863200 & 0.90471200 \\
\hline $\mathrm{C}$ & -7.65631200 & -4.11008000 & 0.07645600 & $\mathrm{C}$ & 4.32618800 & 2.07428800 & 0.18425900 \\
\hline S & -6.52414300 & 1.81814000 & -0.53412700 & C & 3.74197000 & 2.06324500 & -1.08558300 \\
\hline $\mathrm{O}$ & -7.03974900 & 2.52088400 & 0.62644600 & $\mathrm{C}$ & 3.38544300 & 0.83581300 & -1.65171100 \\
\hline $\mathrm{O}$ & -6.81195600 & 2.21097300 & -1.89942200 & C & 3.55441600 & -0.35225900 & -0.94962100 \\
\hline $\mathrm{H}$ & -4.49712400 & 2.47317100 & 1.62234900 & $\mathrm{C}$ & 3.51146900 & 3.34404400 & -1.84468400 \\
\hline $\mathrm{H}$ & -4.67259700 & 0.71555000 & 1.39623800 & $\mathrm{~S}$ & 4.10628900 & -1.77787900 & 1.32906100 \\
\hline 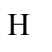 & -2.31462200 & 2.53989100 & -1.07802400 & $\mathrm{O}$ & 4.17338600 & -2.92414300 & 0.43955700 \\
\hline $\mathrm{H}$ & -4.19893400 & 1.75425900 & -2.37820100 & $\mathrm{O}$ & 5.03288700 & -1.57555800 & 2.42828100 \\
\hline $\mathrm{H}$ & -4.22247200 & 0.19017000 & -1.50605200 & $\mathrm{C}$ & -0.78568100 & -0.20755200 & 5.09297200 \\
\hline$\Pi$ & -1.92197700 & 1.35665400 & 2.56939900 & $\mathrm{H}$ & 0.75900600 & -2.02578600 & 3.97701300 \\
\hline $\mathrm{H}$ & -1.79604700 & -0.47837700 & -1.10038600 & $\mathrm{H}$ & 0.15363200 & 0.88025600 & 3.47589900 \\
\hline $\mathrm{H}$ & -2.08176900 & 0.45998400 & -3.39761300 & $\mathrm{H}$ & 2.86759400 & -0.90113100 & 3.87921400 \\
\hline $\mathrm{H}$ & -0.90343100 & 1.73833800 & -3.03263100 & $\mathrm{H}$ & 2.31032900 & 0.20710100 & 2.59362500 \\
\hline 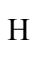 & -0.38540900 & 0.04237300 & -3.07708700 & $\mathrm{H}$ & 4.94049600 & 0.90129100 & 1.89557700 \\
\hline $\mathrm{H}$ & -6.74145700 & -0.28776300 & -2.46773000 & $\mathrm{H}$ & 4.62732700 & 3.02084400 & 0.62353800 \\
\hline $\mathrm{H}$ & -7.16213600 & -2.72672500 & -2.20819200 & $\mathrm{H}$ & 2.96778700 & 0.81655500 & -2.65485000 \\
\hline $\mathrm{H}$ & -7.64816400 & -2.19666200 & 2.02007000 & $\mathrm{H}$ & 3.28721100 & -1.30971400 & -1.39032900 \\
\hline $\mathrm{H}$ & -7.23223300 & 0.24486400 & 1.76322200 & $\mathrm{H}$ & 2.60626300 & 3.27148800 & -2.45522800 \\
\hline $\mathrm{H}$ & -8.31300400 & -4.48132700 & -0.71518900 & $\mathrm{H}$ & 3.40112100 & 4.19052200 & -1.16186600 \\
\hline $\mathrm{H}$ & -6.71439800 & -4.66528900 & 0.01011200 & $\mathrm{H}$ & 4.34972600 & 3.55909100 & -2.51643400 \\
\hline $\mathrm{H}$ & -8.11470500 & -4.34231700 & 1.04044400 & $\mathrm{H}$ & 0.02268000 & -0.04253400 & 5.81055400 \\
\hline $\mathrm{O}$ & 0.80791900 & 0.20302700 & -0.92279600 & $\mathrm{H}$ & -1.55963500 & 0.54322200 & 5.28041500 \\
\hline $\mathrm{C}$ & 2.58746700 & 1.51364500 & 0.28730800 & $\mathrm{H}$ & -1.21062700 & -1.19782200 & 5.28733400 \\
\hline $\mathrm{C}$ & 2.95755700 & 2.39783000 & -0.72646600 & $\mathrm{H}$ & 1.53179100 & -1.43648400 & 0.22144400 \\
\hline
\end{tabular}




\begin{tabular}{|c|c|c|c|c|c|c|c|}
\hline $\mathrm{C}$ & 3.38129100 & 3.69895500 & -0.49840100 & $\mathrm{H}$ & 1.46236900 & -3.12793300 & 0.77844200 \\
\hline $\mathrm{C}$ & 3.43289900 & 4.17902300 & 0.80408800 & $\mathrm{H}$ & -0.94277400 & -3.66407700 & 2.19812900 \\
\hline $\mathrm{C}$ & 3.05260100 & 3.35044800 & 1.84716700 & $\mathrm{H}$ & -2.87980700 & -2.01586400 & 3.08535500 \\
\hline $\mathrm{C}$ & 2.64059000 & 2.05206400 & 1.56626800 & $\mathrm{H}$ & -2.03668600 & 0.46947200 & 2.46067000 \\
\hline $\mathrm{C}$ & 3.08135300 & -0.78175900 & -1.15116800 & $\mathrm{~B}$ & -1.10438500 & 0.01102600 & -0.13220600 \\
\hline $\mathrm{C}$ & 2.59944500 & -1.90604200 & -1.81744000 & $\mathrm{C}$ & -0.29237400 & -0.77389800 & -1.30657100 \\
\hline $\mathrm{C}$ & 3.37139400 & -2.68155800 & -2.67118700 & $\mathrm{C}$ & 0.32286800 & -0.12665500 & -2.37944600 \\
\hline $\mathrm{C}$ & 4.70327300 & -2.34707000 & -2.86990400 & $\mathrm{C}$ & -0.18236400 & -2.16456500 & -1.35843800 \\
\hline $\mathrm{C}$ & 5.23416500 & -1.24824500 & -2.21220400 & $\mathrm{C}$ & 0.99124500 & -0.79912200 & -3.40124900 \\
\hline $\mathrm{C}$ & 4.42424300 & -0.49673100 & -1.36775800 & $\mathrm{C}$ & 0.49146200 & -2.87324500 & -2.33632200 \\
\hline $\mathrm{C}$ & 0.75292900 & -1.82587500 & 1.35992700 & $\mathrm{C}$ & 1.09045000 & -2.17829000 & -3.38081800 \\
\hline $\mathrm{C}$ & 1.86387300 & -1.03012700 & 1.12350700 & $\mathrm{C}$ & -0.70423800 & 1.58654700 & -0.09281100 \\
\hline $\mathrm{C}$ & 2.95664900 & -1.29367400 & 1.94688700 & $\mathrm{C}$ & -1.48582900 & 2.59102100 & -0.66143300 \\
\hline $\mathrm{C}$ & 2.95606500 & -2.25339400 & 2.94619100 & $\mathrm{C}$ & 0.52917300 & 2.01867400 & 0.38583600 \\
\hline $\mathrm{C}$ & 1.81225400 & -3.01748800 & 3.14844200 & $\mathrm{C}$ & -1.11243700 & 3.92694200 & -0.70337200 \\
\hline $\mathrm{C}$ & 0.70051700 & -2.80011500 & 2.35173200 & $\mathrm{C}$ & 0.92669300 & 3.34992700 & 0.40167000 \\
\hline B & 2.07722400 & 0.00683500 & -0.13703300 & $\mathrm{C}$ & 0.10530400 & 4.31179400 & -0.16256000 \\
\hline $\mathrm{F}$ & -0.38311100 & -1.66594900 & 0.64278800 & $\mathrm{C}$ & -2.72309900 & -0.26570500 & -0.10538600 \\
\hline $\mathrm{F}$ & -0.40882600 & -3.51697000 & 2.54092900 & $\mathrm{C}$ & -3.60241600 & 0.56367900 & 0.60460500 \\
\hline $\mathrm{F}$ & 1.78350900 & -3.94235300 & 4.10363100 & $\mathrm{C}$ & -3.38698100 & -1.27171200 & -0.81601300 \\
\hline $\mathrm{F}$ & 4.02945900 & -2.45564900 & 3.70667900 & $\mathrm{C}$ & -4.97471600 & 0.39142500 & 0.68146300 \\
\hline $\mathrm{F}$ & 4.08187900 & -0.58782500 & 1.77366400 & $\mathrm{C}$ & -4.76370400 & -1.48114100 & -0.77232300 \\
\hline $\mathrm{F}$ & 1.32867000 & -2.30274900 & -1.63402500 & $\mathrm{C}$ & -5.56840700 & -0.65126500 & -0.01379100 \\
\hline $\mathrm{F}$ & 2.85280400 & -3.74562400 & -3.28681300 & $\mathrm{~F}$ & 1.42681900 & 1.13381400 & 0.85067500 \\
\hline $\mathrm{F}$ & 5.46351600 & -3.07537200 & -3.68428100 & $\mathrm{~F}$ & 2.09475400 & 3.71366900 & 0.93547100 \\
\hline $\mathrm{F}$ & 6.51410200 & -0.92260700 & -2.39173500 & $\mathrm{~F}$ & 0.48350100 & 5.58487600 & -0.18480200 \\
\hline $\mathrm{F}$ & 5.01247800 & 0.54504300 & -0.76470300 & $\mathrm{~F}$ & -1.90618700 & 4.83557800 & -1.26424200 \\
\hline $\mathrm{F}$ & 2.92728800 & 1.99600000 & -2.00243100 & $\mathrm{~F}$ & -2.65900800 & 2.29865700 & -1.23579200 \\
\hline $\mathrm{F}$ & 3.73316400 & 4.49135600 & -1.50906400 & $\mathrm{~F}$ & -0.72531700 & -2.90385200 & -0.37804700 \\
\hline $\mathrm{F}$ & 3.82426900 & 5.42636100 & 1.04418500 & $\mathrm{~F}$ & 0.56905400 & -4.19919200 & -2.28065900 \\
\hline $\mathrm{F}$ & 3.06315400 & 3.80554400 & 3.09963800 & $\mathrm{~F}$ & 1.73809600 & -2.82656800 & -4.33784000 \\
\hline $\mathrm{F}$ & 2.22541100 & 1.33353600 & 2.63163700 & $\mathrm{~F}$ & 1.57391000 & -0.10190500 & -4.37757300 \\
\hline $\mathrm{C}$ & -0.27627700 & 0.79054400 & -0.44332900 & $\mathrm{~F}$ & 0.31861400 & 1.20520500 & -2.51480500 \\
\hline $\mathrm{H}$ & 0.33998300 & 0.95062600 & 1.63645000 & $\mathrm{~F}$ & -2.74760500 & -2.11235500 & -1.63380000 \\
\hline \multirow[t]{2}{*}{$\mathrm{H}$} & -0.10880500 & 1.98645400 & -0.30594900 & $\mathrm{~F}$ & -5.30554900 & -2.47394400 & -1.47229200 \\
\hline & & & & $\mathrm{F}$ & -5.72113800 & 1.22892000 & 1.39763500 \\
\hline 12 & & & & $\mathrm{~F}$ & -3.14426500 & 1.63395800 & 1.28346200 \\
\hline $\mathrm{N}$ & 0.20954900 & -1.45912200 & -0.09993300 & $\mathrm{~F}$ & -6.88006800 & -0.83891000 & 0.04122500 \\
\hline $\mathrm{C}$ & 0.58194000 & -0.95548900 & -1.43279800 & & & & \\
\hline $\mathrm{C}$ & 1.89873400 & -0.26306200 & -1.16052200 & 10 & & & \\
\hline $\mathrm{C}$ & 2.25379700 & -0.42967500 & 0.30079000 & $\mathrm{~N}$ & 4.87053100 & -1.91916000 & -0.47385900 \\
\hline $\mathrm{C}$ & 0.88507200 & -0.67839700 & 0.94339700 & $\mathrm{C}$ & 4.27778400 & -1.70898300 & 0.84559400 \\
\hline $\mathrm{C}$ & 2.60988200 & 0.48113800 & -2.00174000 & $\mathrm{C}$ & 2.83019800 & -1.53880800 & 0.53745000 \\
\hline $\mathrm{C}$ & 3.88449800 & 1.16374200 & -1.58253400 & $\mathrm{C}$ & 2.62218600 & -1.54536500 & -0.93670700 \\
\hline
\end{tabular}




\begin{tabular}{|c|c|c|c|c|c|c|c|}
\hline $\mathrm{C}$ & 3.02032300 & 0.78359700 & 0.83578600 & $\mathrm{C}$ & 4.02669400 & -1.28274000 & -1.49341700 \\
\hline $\mathrm{C}$ & 3.46396600 & 0.63320800 & 2.28322500 & C & 1.80754700 & -1.33967900 & 1.43331600 \\
\hline $\mathrm{C}$ & -2.32695500 & -0.44410000 & 0.10224100 & $\mathrm{C}$ & 0.55131300 & -1.00949800 & 0.92477900 \\
\hline C & -2.48678700 & 0.29651200 & 1.27150600 & $\mathrm{C}$ & 1.48766200 & -0.61101600 & -1.36615500 \\
\hline $\mathrm{C}$ & -3.18974900 & 1.49441200 & 1.22113500 & $\mathrm{C}$ & 1.19678000 & -0.68855100 & -2.85729700 \\
\hline $\mathrm{C}$ & -3.73848800 & 1.95850900 & 0.02152400 & $\mathrm{C}$ & 6.85939900 & -0.04138400 & -0.36741700 \\
\hline $\mathrm{C}$ & -3.57033700 & 1.19213600 & -1.13489000 & $\mathrm{C}$ & 6.82627800 & 0.80838200 & -1.47232200 \\
\hline $\mathrm{C}$ & -2.86978500 & -0.00882700 & -1.10444400 & $\mathrm{C}$ & 7.01551800 & 2.17091500 & -1.27763500 \\
\hline $\mathrm{S}$ & -1.35911100 & -1.93677500 & 0.14323400 & 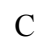 & 7.23552700 & 2.69424100 & 0.00136400 \\
\hline $\mathrm{O}$ & -1.72132700 & -2.72424700 & -1.02193000 & $\mathrm{C}$ & 7.28087500 & 1.81629400 & 1.08855400 \\
\hline $\mathrm{O}$ & -1.41664000 & -2.45996600 & 1.49692000 & $\mathrm{C}$ & 7.09417000 & 0.44914000 & 0.91587600 \\
\hline $\mathrm{H}$ & 0.65712100 & -1.79286200 & -2.13055400 & $\mathrm{C}$ & 7.40703200 & 4.17655300 & 0.20252100 \\
\hline $\mathrm{H}$ & -0.15770000 & -0.24013100 & -1.82450600 & $\mathrm{~S}$ & 6.53682100 & -1.77120400 & -0.59486300 \\
\hline $\mathrm{H}$ & 2.86240800 & -1.33662900 & 0.43252100 & $\mathrm{O}$ & 7.05436200 & -2.48584700 & 0.55736900 \\
\hline $\mathrm{H}$ & 0.91887000 & -1.25375700 & 1.86929700 & $\mathrm{O}$ & 6.85442100 & -2.12002100 & -1.96439000 \\
\hline $\mathrm{H}$ & 0.37804600 & 0.28510900 & 1.12833900 & 10 & 4.49325800 & -2.54218200 & 1.52229300 \\
\hline $\mathrm{H}$ & 2.28775600 & 0.61138900 & -3.03329800 & $\mathrm{H}$ & 4.62608400 & -0.77831600 & 1.33557100 \\
\hline $\mathrm{H}$ & 2.35081000 & 1.65268700 & 0.72723900 & $\mathrm{H}$ & 2.33133100 & -2.58438700 & -1.18863500 \\
\hline $\mathrm{H}$ & 2.60057300 & 0.49535600 & 2.94122400 & $\mathrm{H}$ & 4.20782000 & -1.74225400 & -2.46499800 \\
\hline H & 4.12638500 & -0.23086600 & 2.39065000 & $\mathrm{H}$ & 4.20338300 & -0.19590100 & -1.56187700 \\
\hline $\mathrm{H}$ & 4.01882400 & 1.51398700 & 2.61316400 & $\mathrm{H}$ & 1.97676300 & -1.37089700 & 2.50487700 \\
\hline 11 & -2.08412300 & -0.08180700 & 2.20624400 & $\mathrm{H}$ & 1.74947100 & 0.42091900 & -1.09639400 \\
\hline H & -3.32413800 & 2.07588700 & 2.12947800 & $\mathrm{H}$ & 2.07468600 & -0.39368300 & -3.44086300 \\
\hline $\mathrm{H}$ & -4.00136000 & 1.53762500 & -2.07085600 & $\mathrm{H}$ & 0.91368700 & -1.70689200 & -3.14746700 \\
\hline $\mathrm{H}$ & -2.76138000 & -0.62224300 & -1.99378600 & $\mathrm{H}$ & 0.37171800 & -0.01749400 & -3.10057000 \\
\hline $\mathrm{O}$ & 5.33038400 & 1.16282500 & 0.31537500 & $\mathrm{H}$ & 6.68655600 & 0.39517000 & 76400 \\
\hline $\mathrm{C}$ & 4.19989400 & 1.04157100 & -0.09481300 & $\mathrm{H}$ & 7.00314600 & 2.84036900 & -2.13317400 \\
\hline $\mathrm{H}$ & 4.75210400 & 0.78341400 & -2.13374600 & $\mathrm{H}$ & 7.47676900 & 2.20876500 & 2.08256800 \\
\hline $\mathrm{H}$ & 3.83305300 & 2.23744500 & -1.81460800 & $\mathrm{H}$ & 7.16601700 & -0.23996400 & 1.75217500 \\
\hline $\mathrm{C}$ & -4.53059300 & 3.23959000 & -0.01502900 & & 7.89588600 & 4.63943400 & -0.65835000 \\
\hline $\mathrm{H}$ & -4.47430500 & 3.71284300 & -0.99870300 & $\mathrm{H}$ & 6.43236700 & 4.66051600 & 0.32927300 \\
\hline $\mathrm{H}$ & -5.58725200 & 3.04491600 & 0.19883100 & $\mathrm{H}$ & 8.00181200 & 4.39078300 & 1.09371000 \\
\hline \multirow[t]{2}{*}{$\mathrm{H}$} & -4.16855300 & 3.95181000 & 0.73093400 & $\mathrm{O}$ & -0.79482800 & -0.18649600 & -0.92927500 \\
\hline & & & & $\mathrm{C}$ & -2.54339900 & -1.49624700 & 0.36042200 \\
\hline 13 & & & & $\mathrm{C}$ & -2.90347700 & -2.44179400 & -0.59960200 \\
\hline $\mathrm{Si}$ & 0.00994100 & 0.01229800 & 2.30440200 & $\mathrm{C}$ & -3.30781100 & -3.73351300 & -0.29758900 \\
\hline $\mathrm{C}$ & 1.84434700 & 0.15307900 & 2.76090200 & $\mathrm{C}$ & -3.35134000 & -4.13949400 & 1.03092100 \\
\hline $\mathrm{H}$ & 2.36238100 & -0.62073800 & 2.18061300 & C & -2.98130600 & -3.24808600 & 2.02361500 \\
\hline $\mathrm{C}$ & 2.10256300 & -0.15467900 & 4.24613600 & $\mathrm{C}$ & -2.58666800 & -1.96139000 & 1.66814000 \\
\hline $\mathrm{H}$ & 3.16675300 & -0.02137700 & 4.47376700 & $\mathrm{C}$ & -3.08485000 & 0.73082900 & -1.17173100 \\
\hline $\mathrm{H}$ & 1.54203400 & 0.50922400 & 4.91294600 & $\mathrm{C}$ & -2.63496000 & 1.83939200 & -1.88556700 \\
\hline $\mathrm{H}$ & 1.84018200 & -1.18583600 & 4.50156900 & $\mathrm{C}$ & -3.43179200 & 2.56505500 & -2.76054900 \\
\hline C & -0.78429500 & -1.64257000 & 2.77626900 & C & -4.75612200 & 2.19253400 & -2.93773100 \\
\hline $\mathrm{H}$ & -1.71399300 & -1.70719700 & 2.19724300 & $\mathrm{C}$ & -5.25492600 & 1.10575100 & -2.23685800 \\
\hline
\end{tabular}




\begin{tabular}{|c|c|c|c|c|c|c|c|}
\hline $\mathrm{C}$ & -1.17908100 & -1.70416500 & 4.26194000 & C & -4.42137500 & 0.40612600 & -1.37143 \\
\hline $\mathrm{H}$ & -1.60211500 & -2.68885800 & 4.49319000 & C & -0.69432900 & 1.77688300 & 1.3874270 \\
\hline $\mathrm{H}$ & -0.32245500 & -1.55432600 & 4.92765300 & $\mathrm{C}$ & -1.84860600 & 1.07059500 & 1.0927310 \\
\hline $\mathrm{H}$ & -1.93544900 & -0.95534800 & 4.51566100 & $\mathrm{C}$ & -2.96110500 & 1.43796200 & \\
\hline C & -1.02593000 & 1.53123100 & 2.76535500 & C & -2.94417700 & 2.42898300 & 2.815027 \\
\hline $\mathrm{H}$ & -0.61983100 & 2.36430400 & 2.17822800 & $\mathrm{C}$ & -1.75820500 & 3.11056100 & 3.065918 \\
\hline C & -0.87946700 & 1.91707300 & 4.24748400 & C & -0.61943900 & 2.77606300 & 2.351998 \\
\hline $\mathrm{H}$ & -1.52456700 & 2.77429400 & 4.47352500 & B & -2.04627700 & -0.00718600 & -0.14586600 \\
\hline $\mathrm{H}$ & -1.17160200 & 1.10417000 & 4.92090700 & $\mathrm{~F}$ & 0.47895500 & 1.47791100 & 0.77025 \\
\hline $\mathrm{H}$ & 0.14659900 & 2.20528800 & 4.49545100 & $\mathrm{~F}$ & 0.53436900 & 3.40177200 & 2.598245 \\
\hline $\mathrm{H}$ & 0.00057900 & 0.00729900 & 0.73818700 & $\mathrm{~F}$ & -1.71341200 & 4.06189400 & 3.994993 \\
\hline 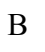 & -0.00205200 & -0.00309200 & -0.83979900 & $\mathrm{~F}$ & -4.04039000 & 2.73495600 & 3.506070 \\
\hline C & -1.00832700 & 1.22017200 & -1.03602200 & $\mathrm{~F}$ & -4.11986800 & 0.79761400 & 844 \\
\hline C & -0.73335300 & 2.46253000 & -0.46665900 & F & -1.37292200 & 2.27457100 & -1.7339250 \\
\hline $\mathrm{C}$ & -2.23541900 & 1.13172000 & -1.69078500 & $\mathrm{~F}$ & -2.94187300 & 3.61772200 & -3.41910600 \\
\hline C & -1.61288600 & 3.53127400 & -0.48120300 & F & -5.54021700 & 2.87278900 & -3.771 \\
\hline C & -3.14135800 & 2.18493400 & -1.74712800 & F & -6.52823300 & 0.74291800 & -2.39669400 \\
\hline $\mathrm{C}$ & -2.83149400 & 3.38935200 & -1.13286500 & $\mathrm{~F}$ & -4.98359200 & -0.62572700 & -0.72666000 \\
\hline $\mathrm{C}$ & 1.56093400 & 0.25367100 & -1.03566100 & $\mathrm{~F}$ & -2.87503500 & -2.11359700 & -1.89706200 \\
\hline C & 2.49820400 & -0.60 & -0.4 & $\mathrm{~F}$ & -3.6 & -4.5 & 100 \\
\hline $\mathrm{C}$ & 2.09930500 & 1.35417600 & -1.69994100 & $\mathrm{~F}$ & -3.72564100 & -5.37697200 & 1.34235400 \\
\hline$c$ & 3.86414700 & -0.37885300 & -0.47927200 & $\mathrm{~F}$ & -2.98606600 & -3.62970800 & 3.30089400 \\
\hline $\mathrm{C}$ & 3.46491300 & 1.60814300 & -1.76126700 & $\mathrm{~F}$ & -2.18403200 & -1.17881700 & 2.69285800 \\
\hline C & 4.35209600 & 0.74049000 & -1.14153900 & $\mathrm{C}$ & 0.24275100 & -0.96555200 & -0.5294740 \\
\hline $\mathrm{C}$ & -0.56072300 & -1.48701700 & -1.02276800 & $\mathrm{H}$ & -0.26080900 & -0.81913300 & 1.62583200 \\
\hline 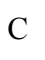 & -1.77117400 & -1.86663900 & -0.44523700 & $\mathrm{H}$ & 0.02735300 & -2.05959300 & -0.70912500 \\
\hline $\mathrm{C}$ & 0.12333800 & -2.50795500 & -1.68021900 & & & & \\
\hline $\mathrm{C}$ & -2.26090300 & -3.16141700 & -0.45712200 & 11 & & & \\
\hline $\mathrm{C}$ & -0.34006000 & -3.81765500 & -1.73424200 & $\mathrm{~N}$ & -4.87011300 & 1.91058000 & -0.52406100 \\
\hline 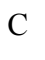 & -1.53562100 & -4.14776500 & -1.11350700 & C & -4.34268900 & 1.78931200 & 0.84347700 \\
\hline $\mathrm{F}$ & 1.28249000 & -2.26846600 & -2.29868200 & C & -2.86552300 & 1.59213900 & 0.59645600 \\
\hline F & 0.35302900 & -4.75316600 & -2.37661100 & C & -2.61121100 & 1.50503600 & -0.89338900 \\
\hline F & -1.98660600 & -5.39448800 & -1.15121300 & $\mathrm{C}$ & -4.00140900 & 1.18790000 & -1.45618500 \\
\hline $\mathrm{F}$ & -3.40547400 & -3.46264300 & 0.15057600 & C & -1.89387200 & 1.41642900 & 1.484653 \\
\hline F & -2.51109500 & -0.95469200 & 0.20587200 & $\mathrm{C}$ & -0.46925100 & 1.22001500 & 1.03663300 \\
\hline F & -2.60612000 & 0.00431200 & -2.30312400 & C & -1.55483100 & 0.44411400 & -1.20224100 \\
\hline F & -4.30087100 & 2.04552200 & -2.38311600 & C & -1.21025700 & 0.28818700 & -2.68013300 \\
\hline F & 0.42689100 & 2.65342700 & 0.18185100 & $\mathrm{C}$ & -6.93704800 & 0.12062000 & -0.34801000 \\
\hline F & -1.30398400 & 4.67602100 & 0.12249400 & $\mathrm{C}$ & -6.86070500 & -0.84950500 & -1.34644700 \\
\hline $\mathrm{F}$ & -3.69032600 & 4.39928400 & -1.16991100 & $\mathrm{C}$ & -7.13387000 & -2.17264700 & -1.02249800 \\
\hline F & 2.08218100 & -1.69667800 & 0.19777600 & C & -7.48250400 & -2.53937600 & 0.28195400 \\
\hline F & 4.69975800 & -1.21631200 & 0.12952600 & $\mathrm{C}$ & -7.56655600 & -1.54420800 & 1.25946500 \\
\hline $\mathrm{F}$ & 5.65684600 & 0.97464000 & -1.18515500 & $\mathrm{C}$ & -7.29580300 & -0.21423400 & 0.95533800 \\
\hline $\mathrm{F}$ & 3.92507000 & 2.67509700 & -2.40795100 & C & -7.75027800 & -3.98162600 & 0.624360 \\
\hline
\end{tabular}




\begin{tabular}{|c|c|c|c|c|c|c|c|}
\hline $\mathrm{F}$ & 1.30927800 & 2.23515000 & $0 \quad-2.31888600$ & $\mathrm{~S}$ & -6.51889000 & 1.80427500 & -0.73518300 \\
\hline c & 0.10494500 & -2.83415600 & 2.39374300 & $\mathrm{O}$ & -7.09987300 & 2.65218600 & 0.2898830 \\
\hline & 1.06190600 & -2.80995000 & 2.92868600 & $\mathrm{O}$ & -6.74727000 & 2.00414200 & 154641 \\
\hline & -0.39221000 & -3.77631600 & 2.65412300 & $\mathrm{H}$ & -4.58004800 & 2.69104400 & 1.41189100 \\
\hline F & 0.32921000 & -2.85569300 & 1.32297800 & $\mathrm{H}$ & -4.76306000 & 0.91885300 & 1.37265800 \\
\hline & 2.42766400 & 1.51895300 & 2.37263900 & $\mathrm{H}$ & -2.28712300 & 2.48274400 & -1.27806900 \\
\hline $\mathrm{H}$ & 1.93198300 & 2.33512100 & 2.91173500 & $\mathrm{H}$ & -4.16969300 & 1.54847300 & 0000 \\
\hline $\mathrm{H}$ & 3.49434300 & 1.56059100 & 2.62368500 & $\mathrm{H}$ & -4.17719700 & 0.09747600 & -1.42194500 \\
\hline $\mathrm{H}$ & 2.32487200 & 1.72390300 & 1.30263500 & $\mathrm{H}$ & -2.08068800 & 1.44504400 & 2.55417800 \\
\hline $\mathrm{C}$ & -2.50282400 & 1.35109200 & 2.38686700 & $\mathrm{H}$ & -1.94839100 & -0.51524500 & -0.81683500 \\
\hline $\mathrm{H}$ & -2.95750500 & 0.51369300 & 2.92952600 & $\mathrm{H}$ & -2.11657200 & 0.06845200 & -3.25038700 \\
\hline $\mathrm{H}$ & -3.07146600 & 2.25371600 & 2.64075500 & $\mathrm{H}$ & -0.76885500 & 4700 & 9200 \\
\hline \multirow[t]{2}{*}{$\mathrm{H}$} & -2.63537800 & 1.15882900 & $1.31^{\circ}$ & $\mathrm{H}$ & -0.49876700 & -0.52621600 & -2.82583700 \\
\hline & & & & $\mathrm{H}$ & -6.62253300 & -0.55660900 & -2.36459300 \\
\hline 14 & & & & $\mathrm{H}$ & -7.08745200 & -2.93457300 & -1.79617300 \\
\hline $\mathrm{N}$ & 1.24664700 & 4.20304300 & 0.09754800 & $\mathrm{H}$ & -7.85852300 & -1.81338100 & 2.27083000 \\
\hline $\mathrm{C}$ & 0.91154800 & -3.49151400 & 1.34411300 & $\mathrm{H}$ & -7.39093400 & 0.56637000 & 1.70394500 \\
\hline$c$ & -0.33866900 & -2.73440900 & 0.96588100 & $\mathrm{H}$ & -8.26121800 & -4.49434200 & -0.19485000 \\
\hline C & -0.70168700 & -3.05880200 & -0.46737300 & 11 & -6.81117900 & -4.51383000 & 1500 \\
\hline $\mathrm{C}$ & 0.63861200 & -3.51685000 & -1.04971300 & $\mathrm{H}$ & -8.36669800 & -4.06826600 & 1.52243700 \\
\hline $\mathrm{C}$ & -0.94267300 & -1.78503400 & 1.67155900 & $\mathrm{O}$ & 0.73789300 & 0.22804800 & -0.79569200 \\
\hline $\mathrm{C}$ & -2.05572700 & -0.95247500 & 1.08815800 & $\mathrm{C}$ & 2.59728200 & 1.48129900 & 0.43091400 \\
\hline C & -2.37196400 & -1.31782200 & -0.31657400 & $\mathrm{C}$ & 2.87328400 & 2.46382000 & -0.51796500 \\
\hline $\mathrm{C}$ & -1.28807000 & -1.82678700 & -1.19072300 & $\mathrm{C}$ & 3.29194300 & 3.74660900 & -0.19773800 \\
\hline $\mathrm{C}$ & -1.70441500 & -2.07874900 & -2.62994100 & $\mathrm{C}$ & 3.44025900 & 4.09376200 & 1.13859200 \\
\hline & 3.74011900 & -3.19829900 & -0.39304400 & $\mathrm{C}$ & 6600 & 31700 & 2.1226800 \\
\hline C & 3.84427000 & -2.73429500 & -1.70396700 & $\mathrm{C}$ & 2.74026800 & 1.88447700 & 1.75445300 \\
\hline $\mathrm{C}$ & 4.46155900 & -1.51345900 & -1.94064000 & $\mathrm{C}$ & 3.04452800 & -0.65547400 & -1.23162600 \\
\hline $\mathrm{C}$ & 4.97599400 & -0.74999600 & -0.88687600 & $\mathrm{C}$ & 4.36952000 & -0.32991900 & -1.50038100 \\
\hline $\mathrm{C}$ & 4.86764400 & -1.24170200 & 0.41808400 & - & 5.13250600 & -0.99205300 & 88600 \\
\hline $\mathrm{C}$ & 4.25107900 & -2.46221600 & 0.67440900 & $\mathrm{C}$ & 4.57150400 & -2.03278200 & -3.18032300 \\
\hline $\mathrm{C}$ & 5.62027400 & 58059400 & -1.16496900 & $\mathrm{C}$ & 3.25494600 & -2.40054500 & -2.93922300 \\
\hline$S$ & 2.83653800 & -4.69449600 & -0.0862310 & $\mathrm{C}$ & 2.53220000 & -1.71777200 & -1.97213700 \\
\hline $\mathrm{O}$ & 3.22740800 & -5.22210700 & 1.20955000 & $\mathrm{C}$ & 3.08300000 & -1.45565500 & 1.81495400 \\
\hline $\mathrm{O}$ & 2.86213700 & -5.49141400 & -1.29970600 & $\mathrm{C}$ & 1.94385100 & -1.12906100 & 1.08070600 \\
\hline 0 & -3.53549800 & -1.22314200 & -0.79065700 & $\mathrm{C}$ & 0.84036400 & -1.93304900 & 1.32855800 \\
\hline $\mathrm{Si}$ & -5.22019100 & -1.17393700 & -0.18144100 & $\mathrm{C}$ & 0.83975400 & -2.97076300 & 2.25526200 \\
\hline $\mathrm{C}$ & -5.43175000 & -2.95430600 & 0.39633100 & ${ }^{2}$ & 1.99570700 & -3.24879300 & 2.96569900 \\
\hline $\mathrm{C}$ & -4.44686300 & -3.38481400 & 1.49067200 & $\mathrm{C}$ & 3.13324700 & -2.48043600 & 2.74484900 \\
\hline C & -6.88162600 & -3.18565300 & 0.85415400 & B & 2.12768500 & 0.01126500 & -0.06999000 \\
\hline $\mathrm{C}$ & -5.48962100 & 0.11086200 & 1.17263900 & $\mathrm{~F}$ & 4.19950600 & -0.74233600 & 1.62078500 \\
\hline C & -6.99238800 & 0.46263400 & 1.17239000 & 1 & 4.25046200 & -2.74109300 & 3.41735500 \\
\hline $\mathrm{C}$ & -4.66483600 & 1.40188800 & 1.06984800 & $\mathrm{~F}$ & 2.01661600 & -4.23656300 & 3.85350900 \\
\hline & -6.10362600 & -0.79946200 & -1.79206600 & $\mathrm{~F}$ & -0.26102000 & -3.69299700 & 2.45778300 \\
\hline
\end{tabular}




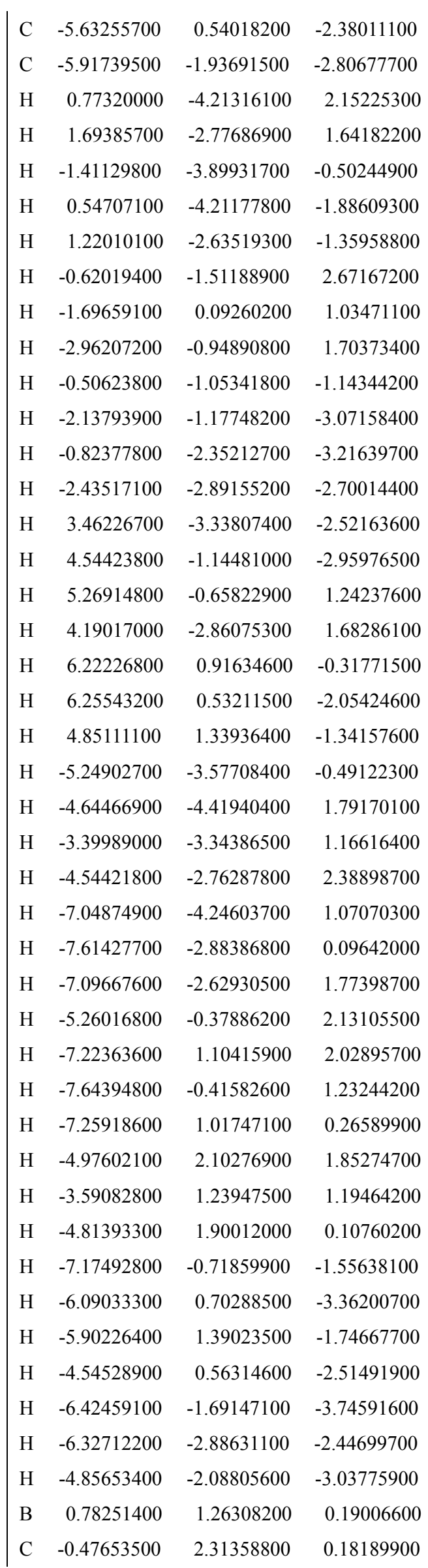

$$
\begin{array}{crrr}
\text { F } & -0.33310100 & -1.72950700 & 0.68713900 \\
\text { F } & 4.98265700 & 0.65628400 & -0.83610700 \\
\text { F } & 6.39628000 & -0.63695600 & -2.67627100 \\
\text { F } & 5.28981100 & -2.67757900 & -4.09388500 \\
\text { F } & 2.70772200 & -3.40618500 & -3.62056000 \\
\text { F } & 1.27198300 & -2.13961400 & -1.75446100 \\
\text { F } & 2.73992400 & 2.17781200 & -1.81993500 \\
\text { F } & 3.54561600 & 4.64117700 & -1.14946600 \\
\text { F } & 3.83402200 & 5.31694800 & 1.47225600 \\
\text { F } & 3.27349000 & 3.48977600 & 3.40657600 \\
\text { F } & 2.43085300 & 1.05443000 & 2.76291200 \\
\text { C } & -0.34429000 & 0.62295800 & -0.33457700 \\
\text { H } & 0.05699500 & 2.18803500 & 0.97698500 \\
\text { H } & 0.12376500 & 0.62832300 & 1.74331400
\end{array}
$$$$
\text { TS7 }
$$$$
\mathrm{N} \quad 7.41600900 \quad-1.72988600 \quad 0.19084400
$$$$
\begin{array}{llll}
\text { C } & 6.96401000 & -1.32298200 & 1.53000200
\end{array}
$$$$
\begin{array}{llll}
\text { C } & 5.49560000 & -1.04887700 & 1.29872200
\end{array}
$$$$
\begin{array}{llll}
\text { C } & 5.16548800 & -1.30221500 & -0.15425900
\end{array}
$$$$
\begin{array}{llll}
\text { C } & 6.53477600 & -1.16111100 & -0.83043300
\end{array}
$$$$
\begin{array}{llll}
\text { C } & 4.60945500 & -0.55297700 & 2.15417100
\end{array}
$$$$
\begin{array}{llll}
\text { C } & 3.17891600 & -0.29939100 & 1.75906300
\end{array}
$$$$
\begin{array}{llll}
\text { C } & 4.09503900 & -0.32976200 & -0.66505800
\end{array}
$$$$
\begin{array}{llll}
\text { C } & 3.72938300 & -0.55079300 & -2.12563100
\end{array}
$$$$
\begin{array}{llll}
\text { C } & 9.58494500 & -0.07825700 & -0.09332000
\end{array}
$$$$
\begin{array}{llll}
\text { C } & 9.51948100 & 0.66374600 & -1.27130700
\end{array}
$$$$
\begin{array}{llll}
\text { C } & 9.88411600 & 2.00466800 & -1.24379900
\end{array}
$$$$
\begin{array}{llll}
\text { C } & 10.31693100 & 2.61066900 & -0.05967600
\end{array}
$$$$
\begin{array}{llll}
\text { C } & 10.38023600 & 1.84046100 & 1.10552100
\end{array}
$$$$
\begin{array}{llll}
\text { C } & 10.01933700 & 0.49760800 & 1.09867800
\end{array}
$$$$
\begin{array}{llll}
\text { S } & 9.05474100 & -1.77477600 & -0.10324900
\end{array}
$$$$
\begin{array}{llll}
\text { O } & 9.63793400 & -2.42916400 & 1.05338600
\end{array}
$$$$
\begin{array}{llll}
\text { O } & 9.19640400 & -2.27663800 & -1.45743100
\end{array}
$$$$
\mathrm{H} \quad 7.15600100 \quad-2.12739900 \quad 2.24341200
$$$$
\mathrm{H} \quad 7.47435700 \quad-0.41254900 \quad 1.88153200
$$$$
\begin{array}{llll}
\mathrm{H} & 4.82171100 & -2.33809100 & -0.28694000
\end{array}
$$$$
\mathrm{H} \quad 6.63829300 \quad-1.72165000 \quad-1.76006600
$$$$
\mathrm{H} \quad 6.75270600 \quad-0.09428400 \quad-1.01861300
$$$$
\mathrm{H} \quad 4.87715600 \quad-0.33361700 \quad 3.18439700
$$$$
\mathrm{H} \quad 4.50643900 \quad 0.68560600 \quad-0.52373400
$$$$
\mathrm{H} \quad 4.62091500 \quad-0.44861300 \quad-2.75009300
$$$$
\mathrm{H} \quad 3.32162100 \quad-1.55558600 \quad-2.27321600
$$$$
\text { H } \quad 2.98601400 \quad 0.17045600 \quad-2.47030000
$$ 


\begin{tabular}{|c|c|c|c|}
\hline & -0.56840700 & 3.45872600 & 0.97091500 \\
\hline & -1.53643700 & 2.13977500 & -0.70156900 \\
\hline & -1.62233500 & 4.36318600 & 0.89164400 \\
\hline & -2.59407500 & 3.02715900 & -0.83568600 \\
\hline & -2.64596900 & 4.14515200 & -0.01899700 \\
\hline & 1.72129900 & 1.61621300 & -1.09358700 \\
\hline & 2.31522500 & 2.86852100 & -1.23140800 \\
\hline & 1.93155200 & 0.75221500 & -2.15715400 \\
\hline $\mathrm{C}$ & 3.05996800 & 3.25325400 & -2.33713800 \\
\hline C & 2.67850100 & 1.08525500 & -3.28335200 \\
\hline$C$ & 3.24468100 & 2.34627800 & -3.37540500 \\
\hline C & 1.56046700 & 1.18316000 & 1.62104900 \\
\hline $\mathrm{C}$ & 0.84148400 & 0.91859900 & 2.78248900 \\
\hline $\mathrm{C}$ & 2.93629700 & 1.21694600 & 1.81264600 \\
\hline C & 1.40610200 & 0.64220100 & 4.01716800 \\
\hline $\mathrm{C}$ & 3.55544900 & 0.94586400 & 3.02933200 \\
\hline C & 2.78869100 & 0.64881100 & 4.14381300 \\
\hline $\mathrm{H}$ & 0.33518000 & 0.14764700 & 0.02597700 \\
\hline $\mathrm{F}$ & 2.17169900 & 3.76654200 & -0.24759500 \\
\hline $\mathrm{F}$ & 3.60098800 & 4.46880200 & -2.41732400 \\
\hline $\mathrm{F}$ & 3.95972300 & 2.68658400 & -4.44620500 \\
\hline F & 2.86066000 & 0.19828000 & -4.26818400 \\
\hline F & 1.39785100 & -0.48906700 & -2.1620120 \\
\hline $\mathrm{F}$ & 0.37662300 & 3.73923500 & 1.87178400 \\
\hline $\mathrm{F}$ & -1.66474000 & 5.43265200 & 1.68413700 \\
\hline $\mathrm{F}$ & -3.67488000 & 4.98714400 & -0.10145900 \\
\hline $\mathrm{F}$ & -3.59389900 & 2.78585700 & -1.69584700 \\
\hline$F$ & -1.59414000 & 1.03373300 & -1.48946400 \\
\hline $\mathrm{F}$ & 3.77159600 & 1.50040800 & 0.80047300 \\
\hline$F$ & 4.88935300 & 0.95241700 & 3.12010400 \\
\hline F & 3.36332900 & 0.37640200 & 5.31357600 \\
\hline $\mathrm{F}$ & 0.63567300 & 0.35529600 & 5.07026100 \\
\hline & -0.51284200 & 0.89771700 & 2.74334200 \\
\hline
\end{tabular}

\section{TS9}

$\begin{array}{llll}\text { C } & -5.29330400 & -2.24534600 & -0.85241900\end{array}$

$\begin{array}{llll}\text { C } & -4.34373200 & -2.23412200 & 0.39580700\end{array}$

$\begin{array}{llll}\text { C } & -4.20947700 & -3.66098100 & 0.87398600\end{array}$

C $\quad-3.50011100 \quad-4.29248100 \quad-0.06332500$

C $\quad-3.15954800 \quad-3.24657900 \quad-1.10789400$

$\begin{array}{llll}\text { C } & -4.42664300 & -2.90045600 & -1.93927700\end{array}$

$\begin{array}{llll}\mathrm{C} & -5.65224000 & -0.77188600 & -0.98787000\end{array}$

$\begin{array}{llll}\mathrm{N} & -5.81368700 & -0.39722400 & 0.41823400\end{array}$

$\begin{array}{llll}\mathrm{C} & -4.82464000 & -1.08441400 & 1.26697300\end{array}$

\begin{tabular}{|c|c|c|c|}
\hline $\mathrm{H}$ & 9.21537000 & 0.18120600 & -2.19525000 \\
\hline $\mathrm{H}$ & 9.84414000 & 2.59010800 & -2.15832500 \\
\hline $\mathrm{H}$ & 10.72757600 & 2.29717100 & 2.02821600 \\
\hline $\mathrm{H}$ & 10.09783100 & -0.11289000 & 1.99302400 \\
\hline $\mathrm{H}$ & 2.50199100 & -1.00514600 & 2.25577400 \\
\hline $\mathrm{H}$ & 2.85453400 & 0.69873800 & 2.08874400 \\
\hline $\mathrm{C}$ & 2.91053400 & -0.36947900 & 0.27297900 \\
\hline $\mathrm{Si}$ & -0.28093900 & -0.30429000 & 0.14162700 \\
\hline $\mathrm{C}$ & -0.01755000 & 0.79664900 & 1.66251900 \\
\hline B & -3.80716300 & 0.08307900 & 0.01875600 \\
\hline $\mathrm{C}$ & -3.84325000 & 1.30592300 & -1.06104400 \\
\hline $\mathrm{C}$ & -2.92612700 & 2.34310400 & -0.96685600 \\
\hline $\mathrm{C}$ & -4.74410600 & 1.44161100 & -2.11391200 \\
\hline $\mathrm{C}$ & -2.84004100 & 3.41128100 & -1.84468800 \\
\hline $\mathrm{C}$ & -4.71034500 & 2.49862700 & -3.01864900 \\
\hline $\mathrm{C}$ & -3.74948100 & 3.49039900 & -2.88864600 \\
\hline $\mathrm{C}$ & -4.36726700 & 0.52132200 & 1.48107800 \\
\hline $\mathrm{C}$ & -5.06252200 & 1.68704000 & 1.78027800 \\
\hline $\mathrm{C}$ & -4.12472400 & -0.30206100 & 2.57562400 \\
\hline $\mathrm{C}$ & -5.46217800 & 2.02763200 & 3.07156300 \\
\hline $\mathrm{C}$ & -4.49296100 & -0.00695600 & 3.87561300 \\
\hline $\mathrm{C}$ & -5.17452500 & 1.17786500 & 4.12605400 \\
\hline $\mathrm{C}$ & -4.50985700 & -1.26596600 & -0.57111300 \\
\hline $\mathrm{C}$ & -4.05700600 & -1.79792200 & -1.77472300 \\
\hline $\mathrm{C}$ & -5.55493400 & -1.98141600 & 0.00575300 \\
\hline $\mathrm{C}$ & -4.54772200 & -2.94981300 & -2.36430900 \\
\hline $\mathrm{C}$ & -6.08945300 & -3.14206900 & -0.54997600 \\
\hline $\mathrm{C}$ & -5.58422000 & -3.63220400 & -1.74186200 \\
\hline $\mathrm{F}$ & -5.59642300 & 2.56842400 & -4.01128700 \\
\hline $\mathrm{F}$ & -5.71539800 & 0.54184200 & -2.31008700 \\
\hline $\mathrm{F}$ & -3.70060100 & 4.50268900 & -3.75166000 \\
\hline $\mathrm{F}$ & -1.89405900 & 4.34386700 & -1.69431600 \\
\hline $\mathrm{F}$ & -2.01441400 & 2.32637000 & 0.03278400 \\
\hline $\mathrm{F}$ & -3.45005400 & -1.45688600 & 2.39140200 \\
\hline $\mathrm{F}$ & -4.19363800 & -0.83209000 & 4.88121100 \\
\hline $\mathrm{F}$ & -5.54316800 & 1.49104800 & 5.36664200 \\
\hline $\mathrm{F}$ & -6.12078100 & 3.16461900 & 3.29834400 \\
\hline $\mathrm{F}$ & -5.39142700 & 2.56714600 & 0.82353300 \\
\hline $\mathrm{F}$ & -6.12671400 & -1.58771500 & 1.15207300 \\
\hline $\mathrm{F}$ & -7.08821000 & -3.78252800 & 0.05806400 \\
\hline $\mathrm{F}$ & -6.07947800 & -4.74352400 & -2.28221500 \\
\hline $\mathrm{F}$ & -4.03196900 & -3.40984400 & -3.50626300 \\
\hline $\mathrm{F}$ & -3.03732500 & -1.19269500 & -2.42164700 \\
\hline $\mathrm{H}$ & -2.57036000 & -0.19584100 & 0.20041400 \\
\hline
\end{tabular}




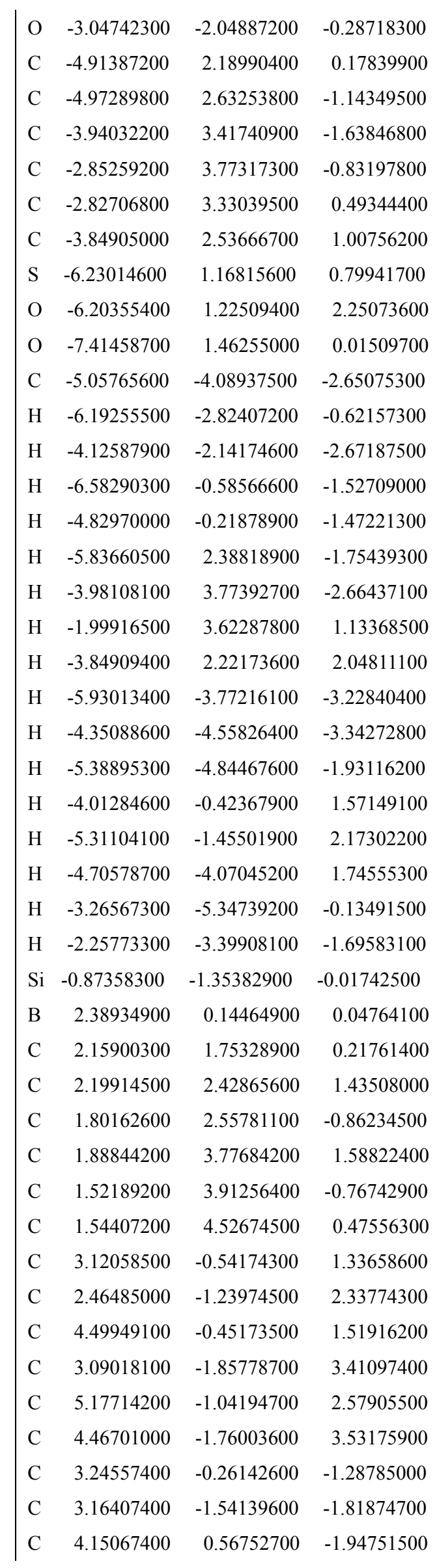

\begin{tabular}{|c|c|c|c|}
\hline $\mathrm{H}$ & 0.70177300 & 0.17641400 & 2.21394100 \\
\hline $\mathrm{C}$ & -0.36438300 & -2.16675900 & 0.49846500 \\
\hline $\mathrm{H}$ & 0.50779500 & -2.55365200 & -0.05122100 \\
\hline $\mathrm{C}$ & -0.47006800 & 0.27372400 & -1.64354800 \\
\hline $\mathrm{H}$ & -1.54164300 & 0.42773300 & -1.79483400 \\
\hline $\mathrm{C}$ & -0.19147600 & -2.50180100 & 1.98800200 \\
\hline $\mathrm{H}$ & -0.11198500 & -3.58694000 & 2.11535400 \\
\hline $\mathrm{H}$ & 0.69986000 & -2.05500000 & 2.44108700 \\
\hline $\mathrm{H}$ & -1.06429600 & -2.17030900 & 2.55838900 \\
\hline $\mathrm{C}$ & 0.24973200 & 1.59443100 & -1.95223100 \\
\hline $\mathrm{H}$ & 1.32657000 & 1.52633000 & -1.75734400 \\
\hline $\mathrm{H}$ & 0.12510800 & 1.83540300 & -3.01435800 \\
\hline $\mathrm{H}$ & -0.14752700 & 2.43231000 & -1.37559700 \\
\hline $\mathrm{C}$ & 0.65435300 & 2.14813200 & 1.37836600 \\
\hline $\mathrm{H}$ & 1.50105800 & 2.07554300 & 0.68533100 \\
\hline $\mathrm{H}$ & -0.05835400 & 2.85367300 & 0.94762200 \\
\hline $\mathrm{H}$ & 1.02105300 & 2.58268300 & 2.31659500 \\
\hline $\mathrm{O}$ & 1.76971500 & -0.41799900 & -0.18908600 \\
\hline $\mathrm{C}$ & -1.21294800 & 0.96775200 & 2.60422500 \\
\hline $\mathrm{H}$ & -1.99710700 & 1.57554100 & 2.15195000 \\
\hline $\mathrm{H}$ & -1.64745400 & 0.00462100 & 2.88034700 \\
\hline $\mathrm{H}$ & -0.88244400 & 1.46394500 & 3.52443600 \\
\hline $\mathrm{C}$ & -1.60386500 & -2.89570400 & -0.03126900 \\
\hline $\mathrm{H}$ & -2.49294600 & -2.60741700 & 0.53408100 \\
\hline $\mathrm{H}$ & -1.79443000 & -2.68737300 & -1.08532800 \\
\hline $\mathrm{H}$ & -1.46522900 & -3.97719900 & 0.07972600 \\
\hline $\mathrm{C}$ & -0.02918100 & -0.82487600 & -2.62440500 \\
\hline $\mathrm{H}$ & -0.59360600 & -1.75064500 & -2.49649500 \\
\hline $\mathrm{H}$ & -0.20175400 & -0.48230000 & -3.65074200 \\
\hline $\mathrm{H}$ & 1.03530100 & -1.05254000 & -2.52290700 \\
\hline $\mathrm{C}$ & 10.74700600 & 4.05428100 & -0.05008000 \\
\hline $\mathrm{H}$ & 11.81394600 & 4.13760700 & -0.28388400 \\
\hline $\mathrm{H}$ & 10.19956200 & 4.63689400 & -0.79531500 \\
\hline $\mathrm{H}$ & 10.58711200 & 4.51004900 & 0.93024700 \\
\hline \multicolumn{4}{|c|}{ TS8 } \\
\hline $\mathrm{N}$ & 4.48408500 & 2.65191700 & 0.59444100 \\
\hline $\mathrm{C}$ & 3.94325800 & 3.02480500 & -0.73114500 \\
\hline $\mathrm{C}$ & 2.54655900 & 2.46762400 & -0.69396300 \\
\hline $\mathrm{C}$ & 2.14758600 & 2.32903300 & 0.75211600 \\
\hline $\mathrm{C}$ & 3.47693300 & 1.96888600 & 1.42855700 \\
\hline $\mathrm{C}$ & 1.81989600 & 2.01720700 & -1.71159600 \\
\hline $\mathrm{C}$ & 0.49738700 & 1.30377300 & -1.51010900 \\
\hline $\mathrm{C}$ & 0.03295400 & 1.54000300 & -0.09906100 \\
\hline
\end{tabular}




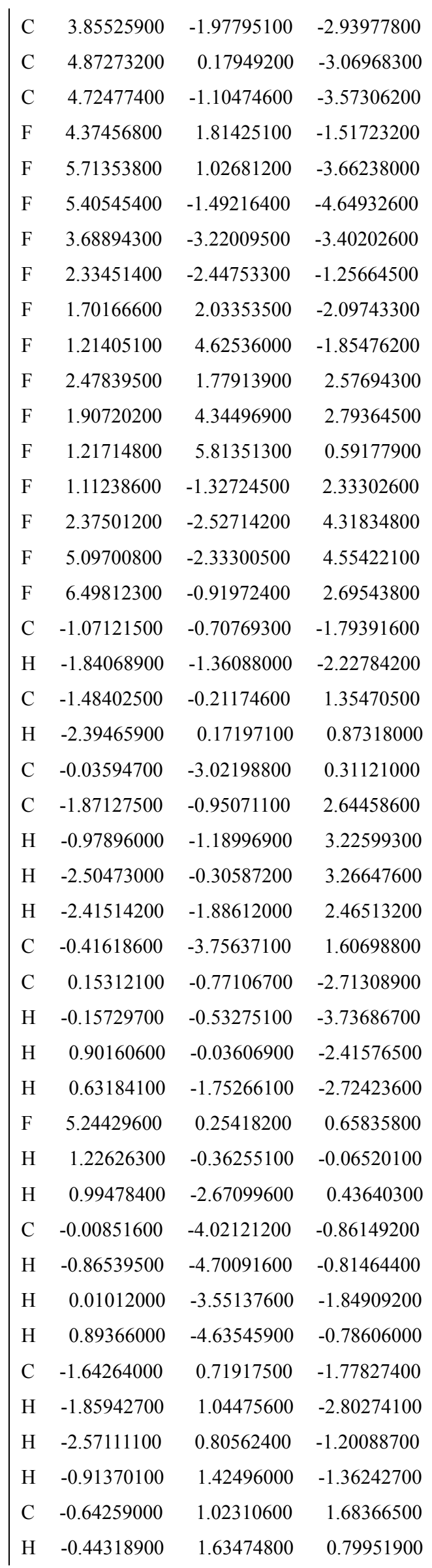

\begin{tabular}{|c|c|c|c|}
\hline $\mathrm{C}$ & 1.03403100 & 1.27972400 & 0.98783400 \\
\hline $\mathrm{C}$ & 0.47699900 & 1.37936500 & 2.40101700 \\
\hline $\mathrm{C}$ & 5.90498700 & 0.44379800 & -0.17703900 \\
\hline $\mathrm{C}$ & 5.55649800 & -0.68960000 & 0.55843500 \\
\hline $\mathrm{C}$ & 5.37791000 & -1.89857400 & -0.10381600 \\
\hline $\mathrm{C}$ & 5.53958300 & -1.99331100 & -1.49136800 \\
\hline $\mathrm{C}$ & 5.91397400 & -0.84785400 & -2.20003300 \\
\hline $\mathrm{C}$ & 6.10136600 & 0.36973400 & -1.55449600 \\
\hline $\mathrm{C}$ & 5.31917300 & -3.30199500 & -2.20501500 \\
\hline $\mathrm{S}$ & 6.02829200 & 2.02101300 & 0.63752700 \\
\hline $\mathrm{O}$ & 6.83493700 & 2.89143200 & -0.19839500 \\
\hline $\mathrm{O}$ & 6.31093700 & 1.78179300 & 2.04079300 \\
\hline $\mathrm{O}$ & -0.91587000 & 2.33021500 & 0.19307900 \\
\hline $\mathrm{Si}$ & -2.20575000 & 3.17050200 & -0.71734100 \\
\hline $\mathrm{C}$ & -2.94117700 & 1.88612800 & -1.89292800 \\
\hline $\mathrm{C}$ & -4.44685900 & 1.65991100 & -1.68724600 \\
\hline $\mathrm{C}$ & -2.64832600 & 2.09215300 & -3.38717600 \\
\hline $\mathrm{C}$ & -3.34213100 & 3.72884700 & 0.67806500 \\
\hline $\mathrm{C}$ & -4.08004000 & 5.02866000 & 0.32116600 \\
\hline $\mathrm{C}$ & -2.61965000 & 3.85622100 & 2.02656500 \\
\hline $\mathrm{C}$ & -1.18821700 & 4.52192500 & -1.56069700 \\
\hline $\mathrm{C}$ & -2.02138100 & 5.44395600 & -2.46496600 \\
\hline $\mathrm{C}$ & -0.36168000 & 5.34528300 & -0.56279700 \\
\hline $\mathrm{H}$ & 3.96143500 & 4.11505100 & -0.83489600 \\
\hline $\mathrm{H}$ & 4.53056300 & 2.59742700 & -1.54992100 \\
\hline $\mathrm{H}$ & 1.80377900 & 3.30250100 & 1.13212500 \\
\hline $\mathrm{H}$ & 3.55446100 & 2.31200400 & 2.46113900 \\
\hline $\mathrm{H}$ & 3.60727600 & 0.87658100 & 1.40820600 \\
\hline $\mathrm{H}$ & 2.18090600 & 2.08106100 & -2.73538900 \\
\hline $\mathrm{H}$ & 0.64413500 & 0.22961600 & -1.66224000 \\
\hline $\mathrm{H}$ & -0.25873200 & 1.62133000 & -2.23097900 \\
\hline $\mathrm{H}$ & 1.46904700 & 0.29421800 & 0.79404500 \\
\hline $\mathrm{H}$ & -0.36241000 & 0.69809600 & 2.54631400 \\
\hline $\mathrm{H}$ & 1.25614500 & 1.10944800 & 3.11901300 \\
\hline $\mathrm{H}$ & 0.14485000 & 2.40068100 & 2.60966200 \\
\hline $\mathrm{H}$ & 5.46065200 & -0.62785700 & 1.63843100 \\
\hline $\mathrm{H}$ & 5.12437100 & -2.78509700 & 0.46883700 \\
\hline $\mathrm{H}$ & 6.07620200 & -0.91459900 & -3.27245400 \\
\hline $\mathrm{H}$ & 6.43264700 & 1.24898900 & -2.09886000 \\
\hline $\mathrm{H}$ & 5.79570800 & -3.29747400 & -3.18845600 \\
\hline $\mathrm{H}$ & 5.72069500 & -4.13851300 & -1.62768000 \\
\hline $\mathrm{H}$ & 4.25104300 & -3.49846900 & -2.35260000 \\
\hline $\mathrm{H}$ & -2.43645400 & 0.96704800 & -1.56936400 \\
\hline $\mathrm{H}$ & -4.80332600 & 0.89917900 & -2.39219500 \\
\hline
\end{tabular}




\begin{tabular}{|c|c|c|c|c|c|c|c|}
\hline $\mathrm{H}$ & -1.19204000 & 1.64280200 & 2.40426000 & $\mathrm{H}$ & -4.67230300 & 1.30818000 & -0.67882600 \\
\hline $\mathrm{H}$ & 0.31076900 & 0.75149100 & 2.13736500 & $\mathrm{H}$ & -5.02301200 & 2.57419100 & -1.87452300 \\
\hline $\mathrm{H}$ & 0.16979200 & -4.68128600 & 1.66397400 & $\mathrm{H}$ & -2.82397000 & 1.15917200 & -3.93282200 \\
\hline $\mathrm{H}$ & -1.47304500 & -4.03573100 & 1.62689700 & $\mathrm{H}$ & -1.62063300 & 2.40736800 & -3.60184600 \\
\hline $\mathrm{H}$ & -0.18824400 & -3.17215500 & 2.49774100 & 11 & -3.31526100 & 2.85016200 & -3.81174400 \\
\hline $\mathrm{C}$ & -1.72392900 & 4.59008600 & -1.39676300 & $\mathrm{H}$ & -4.09100600 & 2.93610400 & 0.79042700 \\
\hline $\mathrm{H}$ & -1.05549000 & 3.96029500 & -1.99393300 & $\mathrm{H}$ & -4.83779700 & 5.25097200 & 1.08018500 \\
\hline $\mathrm{H}$ & -2.09732300 & 5.37978700 & -2.05507900 & $\mathrm{H}$ & -4.59141700 & 4.97308400 & -0.64745600 \\
\hline \multirow[t]{2}{*}{$\mathrm{H}$} & -1.12400700 & 5.05657300 & -0.61080700 & $\mathrm{H}$ & -3.39226400 & 5.88117800 & 0.28845400 \\
\hline & & & & $\mathrm{H}$ & -3.32329800 & 4.19225500 & 2.79628000 \\
\hline \multicolumn{2}{|c|}{$14^{\prime}$} & & & $\mathrm{H}$ & -2.20656000 & 2.89662100 & 2.34809100 \\
\hline $\mathrm{N}$ & -2.02043000 & 4.03695100 & 0.30607400 & $\mathrm{H}$ & -1.79986300 & 4.58342000 & 1.98353500 \\
\hline $\mathrm{C}$ & -1.53364900 & 3.34117500 & 1.50998300 & $\mathrm{H}$ & -0.47852900 & 3.97791700 & -2.20483000 \\
\hline $\mathrm{C}$ & -0.13247300 & 2.94212100 & 1.11638100 & $\mathrm{H}$ & -1.36281600 & 6.09833400 & -3.04684100 \\
\hline $\mathrm{C}$ & 0.15563200 & 3.44378900 & -0.28091400 & $\mathrm{H}$ & -2.64399100 & 4.88984300 & -3.17403900 \\
\hline $\mathrm{C}$ & -1.25079700 & 3.60448400 & -0.86776600 & $\mathrm{H}$ & -2.67901300 & 6.08817400 & -1.87384600 \\
\hline $\mathrm{C}$ & 0.68352600 & 2.13376800 & 1.78099600 & $\Pi$ & 0.30147400 & 6.03793700 & -1.09344400 \\
\hline $\mathrm{C}$ & 1.98353600 & 1.65952300 & 1.19092600 & $\mathrm{H}$ & 0.26031500 & 4.71140700 & 0.07854300 \\
\hline $\mathrm{C}$ & 2.19307300 & 2.07832700 & -0.22211600 & $\mathrm{H}$ & -1.00798700 & 5.94506100 & 0.08762500 \\
\hline $\mathrm{C}$ & 1.03088000 & 2.44880800 & -1.06449100 & B & -1.24545500 & -1.31756800 & 0.19507800 \\
\hline $\mathrm{C}$ & 1.39392700 & 2.88534700 & -2.47505100 & $\mathrm{C}$ & -2.39763800 & -1.68777700 & -0.90357000 \\
\hline $\mathrm{C}$ & -4.19450600 & 2.48669700 & -0.27331200 & $\mathrm{C}$ & -2.29392700 & -1.32469300 & -2.24283400 \\
\hline $\mathrm{C}$ & -4.17695100 & 2.07100400 & -1.60406600 & $c$ & -3.57083000 & -2.37085200 & -0.59068400 \\
\hline $\mathrm{C}$ & -4.48322900 & 0.74973400 & -1.90184400 & $\mathrm{C}$ & -3.26112300 & -1.57302000 & -3.20184600 \\
\hline $\mathrm{C}$ & -4.80370300 & -0.16174600 & -0.88977500 & $\mathrm{C}$ & -4.57623300 & -2.64057100 & -1.51672700 \\
\hline $\mathrm{C}$ & -4.82224400 & 0.28204700 & 0.43642100 & $\mathrm{C}$ & -4.42476800 & -2.23461000 & -2.83194300 \\
\hline $\mathrm{C}$ & -4.52035000 & 1.60224000 & 0.75354900 & $\mathrm{C}$ & -1.92119300 & -1.15585900 & 1.66657300 \\
\hline $\mathrm{C}$ & -5.10845100 & -1.59410800 & -1.23380700 & $\mathrm{C}$ & -1.77866100 & -2.00231700 & 2.75966900 \\
\hline S & -3.68058200 & 4.13984800 & 0.11575200 & $\mathrm{C}$ & -2.76849100 & -0.07765500 & 1.88284300 \\
\hline $\mathrm{O}$ & -4.19859000 & 4.49045400 & 1.42675000 & $\mathrm{C}$ & -2.38136600 & -1.76511500 & 3.99057500 \\
\hline $\mathrm{O}$ & -3.88760900 & 4.96682100 & -1.06012000 & $\mathrm{C}$ & -3.39448000 & 0.20558100 & 3.08588800 \\
\hline $\mathrm{O}$ & 3.34357700 & 2.09857400 & -0.73664300 & $\mathrm{C}$ & -3.19229000 & -0.65094300 & 4.15881800 \\
\hline $\mathrm{Si}$ & 4.99704000 & 1.71523700 & -0.14363600 & $\mathrm{C}$ & 0.10892600 & -2.22993000 & 0.11569400 \\
\hline $\mathrm{C}$ & 5.50687700 & 3.30098900 & 0.70592800 & $\mathrm{C}$ & 1.07388700 & -2.19568600 & 1.12574200 \\
\hline $\mathrm{C}$ & 6.97515800 & 3.21033600 & 1.15706900 & $\mathrm{C}$ & 0.44897900 & -3.05805200 & -0.95626200 \\
\hline $\mathrm{C}$ & 4.95615800 & 0.24678700 & 1.02132500 & $\mathrm{C}$ & 2.25618800 & -2.92021700 & 1.11062600 \\
\hline $\mathrm{C}$ & 6.14957300 & -0.70254000 & 0.81518600 & $\mathrm{C}$ & 1.61979300 & -3.81013300 & -1.01082900 \\
\hline $\mathrm{C}$ & 5.87015600 & 1.44307500 & -1.76514500 & $\mathrm{C}$ & 2.52084400 & -3.75619900 & 0.03732600 \\
\hline $\mathrm{C}$ & 5.93914800 & 2.71203600 & -2.62662700 & $\mathrm{H}$ & -0.85108800 & -0.13991600 & -0.07035500 \\
\hline $\mathrm{H}$ & -1.58345100 & 4.01419200 & 2.36898800 & $\mathrm{~F}$ & -1.03941500 & -3.11365900 & 2.66543600 \\
\hline $\mathrm{H}$ & -2.11898100 & 2.43732500 & 1.73764800 & $\mathrm{~F}$ & -2.19666600 & -2.60355300 & 5.00852300 \\
\hline $\mathrm{H}$ & 0.63891000 & 4.43075400 & -0.23899300 & $\mathrm{~F}$ & -3.77602500 & -0.41118900 & 5.32958800 \\
\hline $\mathrm{H}$ & -1.32467400 & 4.35967800 & -1.65192800 & $\mathrm{~F}$ & -4.17596300 & 1.28223500 & 3.21178900 \\
\hline $\mathrm{H}$ & -1.59761300 & 2.63318700 & -1.25409100 & $\mathrm{~F}$ & -3.01895600 & 0.78143700 & 0.86593400 \\
\hline
\end{tabular}




\begin{tabular}{|c|c|c|c|}
\hline $\mathrm{H}$ & 0.42789500 & 1.73052000 & 2.75619900 \\
\hline $\mathrm{H}$ & 1.96575200 & 0.55408200 & 1.16319600 \\
\hline $\mathrm{H}$ & 2.85610800 & 1.93772800 & 1.79656900 \\
\hline $\mathrm{H}$ & 0.45191200 & 1.50735400 & -1.09861000 \\
\hline $\mathrm{H}$ & 1.98741700 & 2.11810200 & -2.97795700 \\
\hline $\mathrm{H}$ & 0.47838400 & 3.03786700 & -3.05188000 \\
\hline $\mathrm{H}$ & 1.96197800 & 3.82133400 & -2.46996200 \\
\hline $\mathrm{H}$ & -3.94780200 & 2.78512300 & -2.38928900 \\
\hline $\mathrm{H}$ & -4.47056000 & 0.41804800 & -2.93679800 \\
\hline $\mathrm{H}$ & -5.07181300 & -0.41792200 & 1.22934500 \\
\hline $\mathrm{H}$ & -4.56405200 & 1.95873100 & 1.77840000 \\
\hline $\mathrm{H}$ & -5.63068500 & -2.09810500 & -0.41755500 \\
\hline $\mathrm{H}$ & -5.71986000 & -1.65902300 & -2.13859400 \\
\hline $\mathrm{H}$ & -4.17867600 & -2.14220300 & -1.41646400 \\
\hline $\mathrm{H}$ & 4.86028700 & 3.49108700 & 1.57212400 \\
\hline $\mathrm{H}$ & 7.29356700 & 4.12985500 & 1.65490500 \\
\hline $\mathrm{H}$ & 7.12771100 & 2.38639300 & 1.86285700 \\
\hline $\mathrm{H}$ & 7.64597200 & 3.04827200 & 0.30607900 \\
\hline $\mathrm{H}$ & 4.95091700 & 0.62773700 & 2.05133100 \\
\hline $\mathrm{H}$ & 6.11380800 & -1.52430000 & 1.53562600 \\
\hline $\mathrm{H}$ & 7.10741700 & -0.18774800 & 0.94806800 \\
\hline $\mathrm{H}$ & 6.13618100 & -1.14041800 & -0.18603400 \\
\hline $\mathrm{H}$ & 6.87951500 & 1.06428500 & -1.55703800 \\
\hline $\mathrm{H}$ & 6.42029300 & 2.50853100 & -3.58676000 \\
\hline $\mathrm{H}$ & 6.51174400 & 3.50309800 & -2.13112300 \\
\hline $\mathrm{H}$ & 4.93865100 & 3.10512600 & -2.83577700 \\
\hline B & -0.22676600 & -1.17461800 & 0.14641900 \\
\hline $\mathrm{C}$ & 1.24137300 & -1.90261900 & 0.08669600 \\
\hline $\mathrm{C}$ & 1.62354900 & -3.03471700 & 0.80452700 \\
\hline $\mathrm{C}$ & 2.21596800 & -1.41758500 & -0.77607700 \\
\hline $\mathrm{C}$ & 2.87547500 & -3.63184600 & 0.68623600 \\
\hline $\mathrm{C}$ & 3.47099900 & -1.98570500 & -0.93896000 \\
\hline $\mathrm{C}$ & 3.81199800 & -3.10087900 & -0.19074000 \\
\hline $\mathrm{C}$ & -1.06640100 & -1.67191000 & -1.15688800 \\
\hline $\mathrm{C}$ & -1.34438900 & -3.02089700 & -1.36159600 \\
\hline $\mathrm{C}$ & -1.48213600 & -0.82934400 & -2.17664200 \\
\hline $\mathrm{C}$ & -1.98121000 & -3.51599600 & -2.49012600 \\
\hline $\mathrm{C}$ & -2.13254600 & -1.27402400 & -3.32363500 \\
\hline $\mathrm{C}$ & -2.38241800 & -2.62776900 & -3.48229300 \\
\hline $\mathrm{C}$ & -0.98443000 & -1.35112300 & 1.57856400 \\
\hline $\mathrm{C}$ & -0.32844100 & -1.00218500 & 2.75443300 \\
\hline $\mathrm{C}$ & -2.31440300 & -1.70832400 & 1.76507500 \\
\hline $\mathrm{C}$ & -0.92186500 & -0.94816400 & 4.00540400 \\
\hline $\mathrm{C}$ & -2.96072200 & -1.66770200 & 2.99674400 \\
\hline
\end{tabular}

$$
\begin{array}{|lrrr}
\text { F } & -1.19099700 & -0.67833100 & -2.67197900 \\
\text { F } & -3.09180500 & -1.17581600 & -4.46717900 \\
\text { F } & -5.37131500 & -2.48265500 & -3.73218400 \\
\text { F } & -5.67890200 & -3.28953000 & -1.14653500 \\
\text { F } & -3.79596000 & -2.82715400 & 0.64806900 \\
\text { F } & -0.35348600 & -3.19261400 & -2.01936600 \\
\text { F } & 1.88700800 & -4.57503000 & -2.07067100 \\
\text { F } & 3.63559700 & -4.48847700 & 0.00967300 \\
\text { F } & 3.13729400 & -2.81893800 & 2.10817600 \\
\text { F } & 0.89503500 & -1.41616300 & 2.20745600
\end{array}
$$

\section{TS10}

\section{N $\quad-3.94681400$}

$-0.57559400$

0.00692400

C $\quad-3.53397800$

C $\quad-2.13704200$

$-0.24964100$

$-1.36741600$

C $\quad-1.82934700$

C $\quad-3.22493700$

0.28992200

$-1.15366800$

0.28275700

0.32612600

0.27842600

0.95576900

C $\quad-1.31342500$

0.78640300

$-2.07031400$

C $\quad 0.03655800$

1.35970000

$-1.72557900$

C $\quad 0.24406300$

1.54797700

$-0.24636900$

C $\quad-0.93421600$

1.46157700

0.68886300

C $\quad-0.54841800$

1.62960600

2.15961100

C $\quad-6.40537400$

0.63156000

0.06130300

C $\quad-6.54071300$

C $\quad-7.16656400$

1.49905900

1.14140900

2.72670500

0.94750100

C $\quad-7.65717500$

3.09462100

$-0.30738200$

C $\quad-7.51797800$

C $\quad-6.89577600$

2.19878200

$-1.37336700$

0.96884000

$-1.19957300$

C $\quad-8.33183500$

4.42546000

$-0.51847500$

S $\quad-5.54871500$

O $\quad-5.95721500$

$-0.91278200$

0.28630800

O $\quad-5.64601700$

$-1.80316900$

$-0.78469800$

O 1.23038100

$-1.26466700$

1.69105600

2.22907000

0.17848200

Si 2.50587800

3.36522800

$-0.27791800$

C $\quad 3.18852900$

3.10655000

$-2.03005200$

C 2.44448300

3.78505600

$-3.18826200$

C $\quad 4.65002000$

3.59973800

$-2.05221400$

C $\quad 1.55177200$

4.99001500

$-0.07303300$

C $\quad 1.18875500$

5.28362800

1.39073400

C $\quad 0.26285100$

5.04876100

$-0.90881200$

C $\quad 3.81824500$

3.06455500

1.04854700

C $\quad 4.63477200$

4.32810200

1.36827500

C $\quad 3.24384000$

2.42526900

2.32247100

H $\quad-3.57365800$

$-1.14269700$

$-1.99386800$ 


\begin{tabular}{|c|c|c|c|c|c|c|c|}
\hline $\mathrm{C}$ & -2.26398800 & -1.28123800 & 4.12986600 & $\mathrm{H}$ & -4.17750800 & 0.52449200 & -1.81611800 \\
\hline $\mathrm{H}$ & -0.03988900 & 0.01918100 & 0.03617200 & $\mathrm{H}$ & -1.33984700 & -0.65698400 & 0.59423500 \\
\hline $\mathrm{F}$ & -0.97962500 & -3.90707900 & -0.42540100 & $\mathrm{H}$ & -3.26116800 & -0.16622400 & 1.95128800 \\
\hline $\mathrm{F}$ & -2.21383000 & -4.82034800 & -2.63648900 & $\mathrm{H}$ & -3.64434400 & 1.30053100 & 0.99026800 \\
\hline $\mathrm{F}$ & -2.99971200 & -3.07404100 & -4.57446400 & $\mathrm{H}$ & -1.57782100 & 0.77928300 & -3.12456000 \\
\hline $\mathrm{F}$ & -2.52499700 & -0.41031000 & -4.26681800 & $\mathrm{H}$ & 0.14621900 & 2.36217300 & -2.16644100 \\
\hline $\mathrm{F}$ & -1.25775300 & 0.50156700 & -2.11327500 & $\mathrm{H}$ & 0.85684700 & 0.75231500 & -2.13245400 \\
\hline $\mathrm{F}$ & 0.78407700 & -3.60238600 & 1.67294800 & $\mathrm{H}$ & -1.49703100 & 2.36359700 & 0.37006500 \\
\hline $\mathrm{F}$ & 3.19104600 & -4.70330800 & 1.41063800 & $\mathrm{H}$ & -0.42764100 & 2.68575800 & 2.40964800 \\
\hline $\mathrm{F}$ & 5.02701200 & -3.63796200 & -0.29834000 & $\mathrm{H}$ & -1.31822500 & 1.20810300 & 2.80922300 \\
\hline $\mathrm{F}$ & 4.37777300 & -1.41826000 & -1.75190500 & $\mathrm{H}$ & 0.39552700 & 1.12939900 & 2.38014200 \\
\hline $\mathrm{F}$ & 1.98481600 & -0.29447700 & -1.50232500 & $\mathrm{H}$ & -6.18830800 & 1.19493900 & 2.12244600 \\
\hline $\mathrm{F}$ & -3.07539600 & -2.11019600 & 0.73499900 & $\mathrm{H}$ & -7.28786200 & 3.40539200 & 1.78738800 \\
\hline $\mathrm{F}$ & -4.25532000 & -1.98676300 & 3.08786300 & $\mathrm{H}$ & -7.91479500 & 2.46638700 & -2.34944900 \\
\hline $\mathrm{F}$ & -2.86597600 & -1.23242800 & 5.31612900 & $\mathrm{H}$ & -6.81398800 & 0.25531500 & -2.01406500 \\
\hline $\mathrm{F}$ & -0.22224200 & -0.56377200 & 5.07626800 & $\mathrm{H}$ & -7.73077100 & 5.06823100 & -1.17028900 \\
\hline $\mathrm{F}$ & 0.98239900 & -0.66036700 & 2.71450600 & $\mathrm{H}$ & -9.30853000 & 4.29836900 & -0.99495500 \\
\hline $\mathrm{H}$ & 5.36968800 & 4.14624900 & 0.02130000 & $\mathrm{H}$ & -8.48088200 & 4.94999200 & 0.42821700 \\
\hline $\mathrm{H}$ & 5.35282600 & 0.63809000 & -2.29830600 & $\mathrm{H}$ & 3.20128600 & 2.02414200 & -2.21114500 \\
\hline \multirow[t]{2}{*}{$\mathrm{H}$} & 4.02304600 & -0.31550200 & 0.90309700 & $\mathrm{H}$ & 2.97330000 & 3.58962900 & -4.12780700 \\
\hline & & & & $\mathrm{H}$ & 1.41821700 & 3.43178800 & -3.31907600 \\
\hline $3 a^{\prime}$ & & & & $\mathrm{H}$ & 2.41350900 & 4.87257300 & -3.05533200 \\
\hline $\mathrm{N}$ & 1.79786200 & -1.37424100 & -0.21211000 & $\mathrm{H}$ & 5.09650800 & 3.38452800 & -3.02868300 \\
\hline $\mathrm{C}$ & 1.32778300 & -0.98954200 & 1.13286900 & $\mathrm{H}$ & 5.27471400 & 3.11972600 & -1.29668900 \\
\hline $\mathrm{C}$ & 0.14025500 & -0.10740200 & 0.82003300 & $\mathrm{H}$ & 4.69768000 & 4.68614900 & -1.90406600 \\
\hline $\mathrm{C}$ & -0.14614500 & -0.18219300 & -0.66127000 & $\mathrm{H}$ & 2.23266900 & 5.77907900 & -0.43053100 \\
\hline $\mathrm{C}$ & 1.24232100 & -0.47664800 & -1.23569100 & $\mathrm{H}$ & 0.66915600 & 6.24562800 & 1.46712700 \\
\hline $\mathrm{C}$ & -0.48705500 & 0.72088800 & 1.64982300 & $\mathrm{H}$ & 2.05562600 & 5.32039500 & 2.05308400 \\
\hline $\mathrm{C}$ & -1.58505000 & 1.64272200 & 1.18455800 & $\mathrm{H}$ & 0.51002900 & 4.51524900 & 1.77732100 \\
\hline $\mathrm{C}$ & -2.03843600 & 1.35900200 & -0.25194700 & $\mathrm{H}$ & -0.23349800 & 6.01572800 & -0.76847100 \\
\hline $\mathrm{C}$ & -0.83934600 & 1.07915700 & -1.16895300 & $\mathrm{H}$ & 0.42833800 & 4.91784000 & -1.98008400 \\
\hline $\mathrm{C}$ & -1.26574900 & 0.95644000 & -2.62797600 & $\mathrm{H}$ & -0.44926900 & 4.28138600 & -0.57731600 \\
\hline $\mathrm{C}$ & 4.39312400 & -0.49854500 & -0.08858700 & $\mathrm{H}$ & 4.50916000 & 2.33711100 & 0.60746300 \\
\hline $\mathrm{C}$ & 4.70054500 & 0.35212700 & -1.14898700 & $\mathrm{H}$ & 5.46984300 & 4.07067900 & 2.02898200 \\
\hline $\mathrm{C}$ & 5.46923800 & 1.48379000 & -0.90546600 & $\mathrm{H}$ & 5.06003900 & 4.79131000 & 0.47189100 \\
\hline $\mathrm{C}$ & 5.93777500 & 1.77514600 & 0.37970100 & $\mathrm{H}$ & 4.04044600 & 5.08761300 & 1.88420300 \\
\hline $\mathrm{C}$ & 5.62053200 & 0.90239100 & 1.42357000 & $\mathrm{H}$ & 4.04126300 & 2.27865700 & 3.06000500 \\
\hline $\mathrm{C}$ & 4.85261500 & -0.23516800 & 1.19903400 & $\mathrm{H}$ & 2.79841500 & 1.44726800 & 2.11108500 \\
\hline $\mathrm{C}$ & 6.80119700 & 2.98569500 & 0.62265600 & $\mathrm{H}$ & 2.47502800 & 3.05317300 & 2.78785500 \\
\hline S & 3.35057100 & -1.91525400 & -0.37344800 & $\mathrm{~B}$ & 1.36500600 & -1.44099300 & 0.06938000 \\
\hline $\mathrm{O}$ & 3.57261800 & -2.84097700 & 0.72475400 & $\mathrm{C}$ & 2.52348700 & -1.45005300 & -1.07258300 \\
\hline $\mathrm{O}$ & 3.50840900 & -2.29670800 & -1.76675800 & $\mathrm{C}$ & 3.43813400 & -0.40895900 & -1.11993600 \\
\hline $\mathrm{O}$ & -2.88291300 & 0.22354000 & -0.30812100 & $\mathrm{C}$ & 2.73615500 & -2.43681600 & -2.03235200 \\
\hline $\mathrm{Si}$ & -4.51953800 & 0.19938700 & 0.04761500 & $\mathrm{C}$ & 4.48643700 & -0.31257000 & -2.01787300 \\
\hline
\end{tabular}




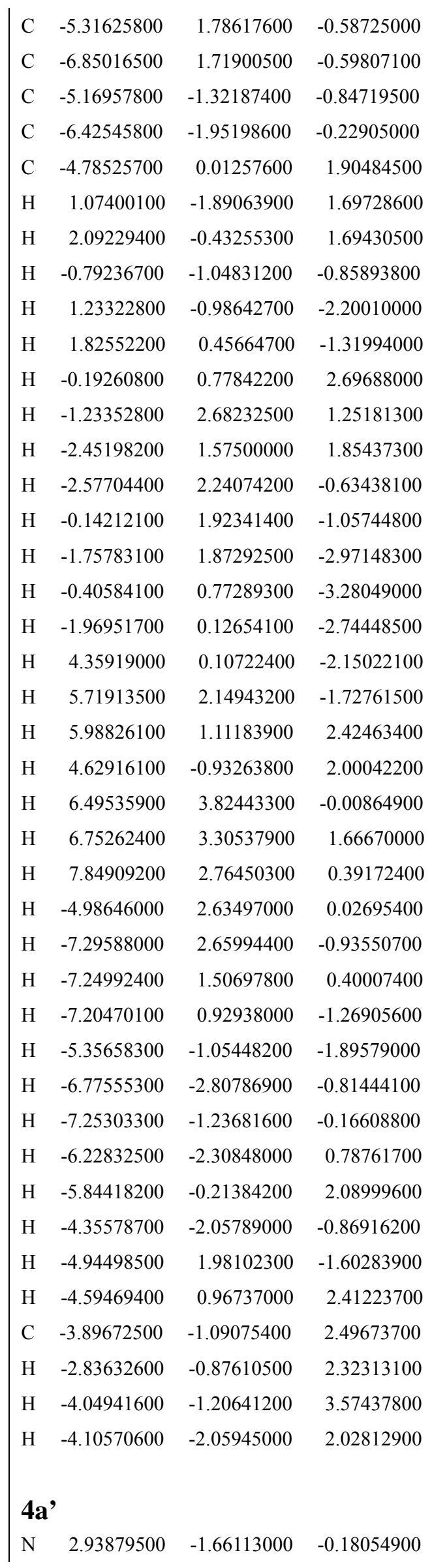

$$
\begin{array}{llll}
\text { C } & 3.76618600 & -2.38291000 & -2.96811200 \\
\text { C } & 4.64794100 & -1.31328600 & -2.96525200 \\
\text { C } & 0.13486700 & -2.45053900 & -0.25429500 \\
\text { C } & -0.46446300 & -3.34533400 & 0.62758500 \\
\text { C } & -0.50523400 & -2.34555700 & -1.48848300 \\
\text { C } & -1.66011300 & -4.00647700 & 0.35391400 \\
\text { C } & -1.69010500 & -2.98451800 & -1.80567300 \\
\text { C } & -2.29328700 & -3.80618700 & -0.86202800 \\
\text { C } & 1.94312800 & -1.45374000 & 1.59497600 \\
\text { C } & 1.07877800 & -1.23386600 & 2.66652900 \\
\text { C } & 3.28234700 & -1.55914100 & 1.96649700 \\
\text { C } & 1.47733700 & -1.07047100 & 3.98260600 \\
\text { C } & 3.73264200 & -1.41581300 & 3.27695300 \\
\text { C } & 2.82738200 & -1.16160300 & 4.29335900 \\
\text { H } & 0.82436100 & -0.26054500 & -0.01181900 \\
\text { F } & 0.08984800 & -3.62413700 & 1.81413300 \\
\text { F } & -2.19710800 & -4.82647300 & 1.25436000 \\
\text { F } & -3.44444200 & -4.40615100 & -1.13595700 \\
\text { F } & -2.27876800 & -2.78493900 & -2.99081900 \\
\text { F } & 0.03158500 & -1.56960500 & -2.45024500 \\
\text { F } & 3.34095700 & 0.57824200 & -0.19951200 \\
\text { F } & 5.32859200 & 0.72735600 & -1.98379300 \\
\text { F } & 5.63568300 & -1.24634200 & -3.85337200 \\
\text { F } & 3.91168200 & -3.35314600 & -3.86843400 \\
\text { F } & 1.95082400 & -3.51587400 & -2.09993100 \\
\hline & 4.24209300 & -1.80824200 & 1.06397500 \\
\text { F } & 3.24169300 & -1.00986100 & 5.54813500 \\
\hline
\end{array}
$$

\section{TS11}

$\begin{array}{lrrr}\mathrm{N} & 6.85729700 & 1.37214600 & -0.63125000 \\ \mathrm{C} & 6.31545700 & 0.40387700 & -1.59796000 \\ \mathrm{C} & 5.03098100 & -0.02993700 & -0.92860400 \\ \mathrm{C} & 4.85377100 & 0.76506600 & 0.34670500 \\ \mathrm{C} & 6.30018700 & 1.13007500 & 0.70039400 \\ \mathrm{C} & 4.22672100 & -1.02751400 & -1.28141000 \\ \mathrm{C} & 3.00554400 & -1.39892200 & -0.47225400 \\ \mathrm{C} & 4.10444600 & -0.04641600 & 1.41928600 \\ \mathrm{C} & 3.84332300 & 0.72877400 & 2.70253300 \\ \mathrm{C} & 9.37990900 & 0.35299400 & -0.30133600 \\ \mathrm{C} & 9.67281300 & 0.12998400 & 1.04297900 \\ \mathrm{C} & 10.34529000 & -1.03228000 & 1.40160800\end{array}$




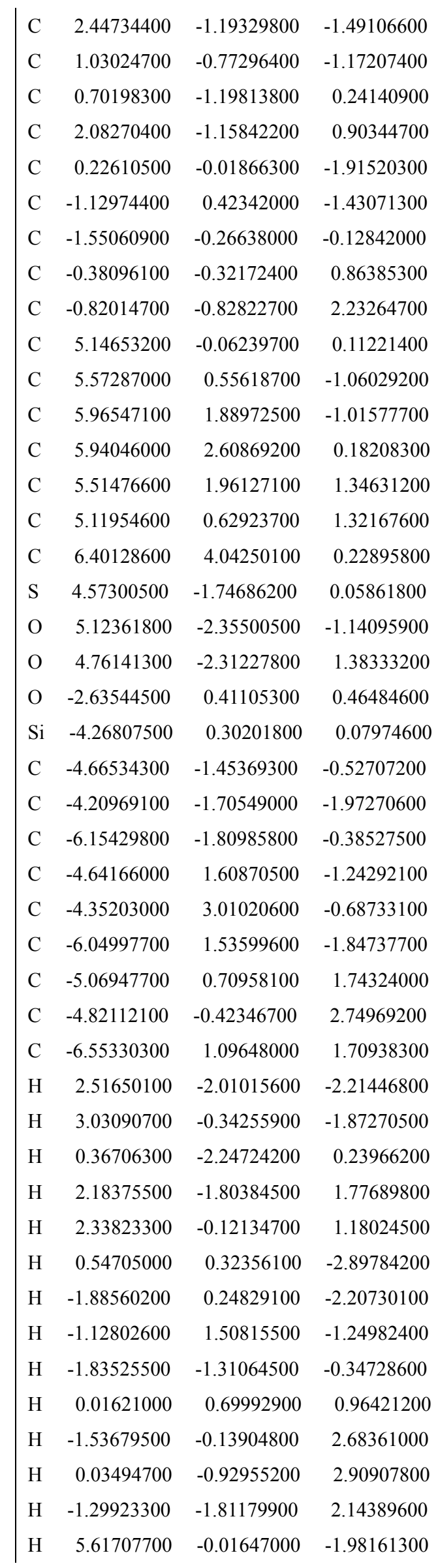

\begin{tabular}{|c|c|c|c|}
\hline C & 10.73246400 & -1.96892700 & 0.43733 \\
\hline $\mathrm{C}$ & 10.43243700 & 1.71565600 & 0.90457300 \\
\hline & 1600 & 2100 & .28368700 \\
\hline & 8.46115500 & 1.80282900 & 0.76257200 \\
\hline $\mathrm{O}$ & 29400 & 2.03813000 & 90100 \\
\hline O & 8.67855600 & 2.81087900 & 3900 \\
\hline $\mathrm{H}$ & 6.16476700 & 0.89364000 & -2.56278500 \\
\hline $\mathrm{H}$ & 400 & 04 & 9000 \\
\hline $\mathrm{H}$ & 4.30769900 & 1.69613300 & 0.13691500 \\
\hline $\mathrm{H}$ & 0. & 0 & $520+2-2$ \\
\hline $\mathrm{H}$ & 6.78 & 00 & 800 \\
\hline $\mathrm{H}$ & 4.41362900 & -1.61810800 & 8600 \\
\hline $\mathrm{H}$ & 4.71269500 & 00 & 0 \\
\hline $\mathrm{H}$ & 4.79112800 & 1.03475400 & .15262800 \\
\hline $\mathrm{H}$ & 3.24 & 0 & 500 \\
\hline $\mathrm{H}$ & 3.29 & 00 & 00 \\
\hline $\mathrm{H}$ & 40156100 & 87563800 & 24700 \\
\hline $\mathrm{H}$ & 0 & 0 & 00 \\
\hline $\mathrm{H}$ & 0.74037900 & -2.42948300 & -1.66366300 \\
\hline $\mathrm{H}$ & 9.55443400 & -0.3 & 00 \\
\hline $\mathrm{H}$ & $20<+2$ & 0 & 00 \\
\hline $\mathrm{H}$ & 3.10287300 & -2.43238600 & 97500 \\
\hline $\mathrm{C}$ & 00 & 700 & 00 \\
\hline $\mathrm{O}$ & 1.75445500 & 0 & 1.19301000 \\
\hline $\mathrm{C}$ & 11.496 & -3.2 & 1400 \\
\hline $\mathrm{H}$ & 1121151000 & -4.02692700 & 012674 \\
\hline $\mathrm{H}$ & .57352400 & -3.00656900 & 00 \\
\hline $\mathrm{H}$ & 01000 & & 900 \\
\hline $\mathrm{Si}$ & 12615500 & 032180800 & 058232700 \\
\hline $\mathrm{O}_{\mathrm{O}}$ & -3.63755700 & -0.03708100 & 6000 \\
\hline $\mathrm{C}$ & -4.20028000 & -0.05851500 & 79200 \\
\hline $\mathrm{C}$ & 6 & 0.67538400 & 00 \\
\hline $\mathrm{C}$ & -3.58521600 & -0.83478300 & 7200 \\
\hline $\mathrm{C}$ & $5=7000-100$ & & \\
\hline$C$ & -3.94906000 & 29900 & 5400 \\
\hline $\mathrm{C}$ & -5.02584000 & -0.09523800 & 4.13585 \\
\hline $\mathrm{C}$ & 400854100 & 126701200 & 2900 \\
\hline$C$ & -3.51662100 & 2.56732600 & 1600 \\
\hline $\mathrm{C}$ & -4 & 1.51058500 & 0 \\
\hline $\mathrm{C}$ & -3.75 & 3.8 & 0 \\
\hline$a^{2}$ & -5.08556200 & 2.73989100 & 72500 \\
\hline $\mathrm{C}$ & -4.55453600 & 3.89951500 & $-2.0492 \varepsilon$ \\
\hline $\mathrm{C}$ & -4.07622700 & 0 & -0 \\
\hline $\mathrm{C}$ & -3.30847300 & -1.73067100 & -2.07980500 \\
\hline
\end{tabular}




\begin{tabular}{|c|c|c|c|c|c|c|c|}
\hline $\mathrm{H}$ & 6.30521600 & 2.37944200 & -1.92461800 & $\mathrm{C}$ & -5.19938200 & -2.12104300 & -0.75699600 \\
\hline $\mathrm{H}$ & 5.50200800 & 2.50789700 & 2.28562600 & $\mathrm{C}$ & -3.57983300 & -2.84030000 & -2.86329500 \\
\hline $\mathrm{H}$ & 4.81699000 & 0.11420200 & 2.22848300 & $\mathrm{C}$ & -5.52231000 & -3.23848500 & -1.52149000 \\
\hline $\mathrm{H}$ & 6.27573600 & 4.53177300 & -0.74032900 & $\mathrm{C}$ & -4.70537700 & -3.60247400 & -2.58093000 \\
\hline $\mathrm{H}$ & 5.84544100 & 4.61325700 & 0.97767300 & $\mathrm{~F}$ & -6.04527400 & -1.82191700 & 0.23807700 \\
\hline $\mathrm{H}$ & 7.46314900 & 4.09749500 & 0.49350600 & $\mathrm{~F}$ & -6.60937700 & -3.96031700 & -1.24753400 \\
\hline $\mathrm{H}$ & -4.09906200 & -2.13093300 & 0.13227700 & $\mathrm{~F}$ & -4.99314000 & -4.67436500 & -3.31767800 \\
\hline $\mathrm{H}$ & -3.13024400 & -1.58203800 & -2.10421100 & $\mathrm{~F}$ & -2.76991100 & -3.18620300 & -3.86793900 \\
\hline $\mathrm{H}$ & -4.46172900 & -2.72638400 & -2.28510500 & $\mathrm{~F}$ & -2.19455600 & -1.03506000 & -2.39724600 \\
\hline $\mathrm{H}$ & -4.70595100 & -1.02114300 & -2.67099800 & $\mathrm{~F}$ & -2.53687300 & -1.61951700 & 2.04329300 \\
\hline $\mathrm{H}$ & -6.49170600 & -1.77698600 & 0.65457000 & $\mathrm{~F}$ & -3.27191100 & -1.61232200 & 4.59563300 \\
\hline $\mathrm{H}$ & -6.34221900 & -2.82292100 & -0.76181200 & $\mathrm{~F}$ & -6.01122300 & 1.43053000 & 1.03741100 \\
\hline $\mathrm{H}$ & -6.79173700 & -1.12856100 & -0.96083900 & $\mathrm{~F}$ & -6.75005600 & 1.40527600 & 3.58725200 \\
\hline $\mathrm{H}$ & -3.92306200 & 1.41920200 & -2.05519700 & $\mathrm{~F}$ & -5.40659600 & -0.10049900 & 5.41174900 \\
\hline $\mathrm{H}$ & -3.33919300 & 3.08022600 & -0.27650900 & $\mathrm{~F}$ & -2.72100800 & 2.54972600 & 0.72936900 \\
\hline $\mathrm{H}$ & -4.45613000 & 3.77321300 & -1.46814300 & $\mathrm{~F}$ & -3.22399400 & 4.91663600 & -0.38718200 \\
\hline $\mathrm{H}$ & -5.05330500 & 3.26838600 & 0.11552900 & $\mathrm{~F}$ & -4.80279700 & 5.08204400 & -2.60920000 \\
\hline $\mathrm{H}$ & -6.22976400 & 0.58406400 & -2.35697400 & $\mathrm{~F}$ & -5.85884400 & 2.80901600 & -3.67283700 \\
\hline $\mathrm{H}$ & -6.19649600 & 2.33582200 & -2.58378400 & $\mathrm{C}$ & 0.20482100 & 0.42947900 & -1.11660000 \\
\hline $\mathrm{H}$ & -6.82729600 & 1.65242400 & -1.08451400 & $\mathrm{H}$ & 1.26195500 & 0.72933500 & -1.12209500 \\
\hline $\mathrm{H}$ & -4.50103400 & 1.58662300 & 2.08986300 & $\mathrm{H}$ & 0.09994200 & -0.36317200 & -1.86780300 \\
\hline $\mathrm{H}$ & -3.76328000 & -0.70168600 & 2.79420300 & $\mathrm{C}$ & -0.13815600 & -2.19959500 & 0.73999000 \\
\hline $\mathrm{H}$ & -5.13414300 & -0.12604700 & 3.75783900 & $\mathrm{H}$ & 0.89668500 & -2.53445700 & 0.59800700 \\
\hline $\mathrm{H}$ & -5.39017500 & -1.32286200 & 2.48692400 & $\mathrm{H}$ & -0.38529600 & -2.43348200 & 1.78102700 \\
\hline $\mathrm{H}$ & -6.72777200 & 2.00724100 & 1.12824000 & $\mathrm{C}$ & -0.66284300 & 0.74732100 & 2.01682600 \\
\hline $\mathrm{H}$ & -6.92540000 & 1.28036300 & 2.724 & $\mathrm{C}$ & -1.05386000 & -2.98512600 & -0.20410800 \\
\hline \multirow[t]{2}{*}{$\mathrm{H}$} & -7.17468400 & 0.30426600 & 1.27639600 & $\mathrm{H}$ & -0.88732900 & -4.05984600 & -0.08578500 \\
\hline & & & & $\mathrm{H}$ & -2.10148800 & -2.78004400 & 0.01708100 \\
\hline \multicolumn{2}{|c|}{ TS12 } & & & $\mathrm{H}$ & -0.87658800 & -2.73042000 & -1.25376300 \\
\hline $\mathrm{N}$ & -3.83028100 & 3.00599400 & -0.16110200 & $\mathrm{C}$ & -0.35509700 & 0.13921500 & 3.39236500 \\
\hline $\mathrm{C}$ & -3.34556300 & 2.75723100 & 1.20723600 & $\mathrm{H}$ & -0.59481800 & 0.85424800 & 4.18454500 \\
\hline $\mathrm{C}$ & -1.86079500 & 2.57752100 & 1.00096000 & $\mathrm{H}$ & -0.95322900 & -0.75822800 & 3.57023700 \\
\hline $\mathrm{C}$ & -1.53108100 & 2.76641700 & -0.46447000 & $\mathrm{H}$ & 0.70212600 & -0.12687700 & 3.49788200 \\
\hline $\mathrm{C}$ & -2.87754400 & 2.47394200 & -1.13927900 & $\mathrm{C}$ & -0.65481200 & 1.63061900 & -1.52769600 \\
\hline $\mathrm{C}$ & -0.95317600 & 2.18273200 & 1.88784100 & $\mathrm{H}$ & -0.20541900 & 2.13755600 & -2.38643200 \\
\hline $\mathrm{C}$ & 0.50090500 & 2.10967400 & 1.50746600 & $\mathrm{H}$ & -1.65244500 & 1.30020600 & -1.81355900 \\
\hline $\mathrm{C}$ & 0.72775200 & 1.92856800 & 0.02850300 & $\mathrm{H}$ & -0.75872100 & 2.36011900 & -0.71894800 \\
\hline $\mathrm{C}$ & -0.44238100 & 1.77332800 & -0.90624300 & $\mathrm{~F}$ & -5.38317000 & 0.45034400 & -2.58187900 \\
\hline $\mathrm{C}$ & -0.03569600 & 1.89471500 & -2.36685200 & $\mathrm{H}$ & -2.38495100 & -0.09906300 & -0.03602200 \\
\hline $\mathrm{C}$ & -5.74487900 & 1.05532200 & -0.09770000 & $\mathrm{H}$ & -0.14417800 & 1.70870400 & 1.89750200 \\
\hline $\mathrm{C}$ & -5.47046900 & 0.09106300 & -1.06635800 & $\mathrm{H}$ & -1.72753800 & 0.96935800 & 1.92881900 \\
\hline $\mathrm{C}$ & -5.62596400 & -1.25277500 & -0.74448000 & & & & \\
\hline $\mathrm{C}$ & -6.05114700 & -1.64489500 & 0.53071600 & $4 a$ & & & \\
\hline $\mathrm{C}$ & -6.33447500 & -0.65463600 & 1.47637600 & $\mathrm{~N}$ & 2.23782300 & -1.33685700 & 0.07894200 \\
\hline
\end{tabular}




\begin{tabular}{|c|c|c|c|c|c|c|c|}
\hline $\mathrm{C}$ & -6.18272500 & 0.69311300 & 1.17437000 & C & 1.79407800 & -0.70041500 & 1.33 \\
\hline C & -6.19783300 & -3.10001000 & 0.89322700 & C & 0.60583600 & 0.11706700 & 0.87947000 \\
\hline & -5.45632800 & 2.77169700 & 0.45123300 & $\mathrm{C}$ & 0.29188700 & -0.24106400 & -0.55561600 \\
\hline & -6.15275900 & 3.55210400 & 0.55585400 & C & 1.67076600 & -0.64171300 & -1.08580800 \\
\hline C & -5.63201500 & 2.98657100 & -1.87660200 & C & -0.00063400 & 1.09679900 & 1.54389000 \\
\hline 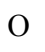 & 1.83468400 & 2.29733900 & -0.47977700 & $\mathrm{C}$ & -1.10188400 & 1.92042200 & 0.92649200 \\
\hline & 3.23746500 & 3.23947700 & 0.05668900 & C & -1.59809200 & 1.33868500 & -0.39885100 \\
\hline C & 2.53590000 & 4.97801700 & -0.06148900 & $\mathrm{C}$ & -0.41864000 & 0.89908000 & -1.27995200 \\
\hline C & 3.51804000 & 6.03685500 & 0.46368200 & $\mathrm{C}$ & -0.87928200 & 0.50981900 & -2.68030600 \\
\hline C & 3.80344900 & 2.88144900 & 1.80847800 & $\mathrm{C}$ & 4.84283400 & -0.48863100 & -0.01539100 \\
\hline C & 4.08024500 & 1.42688600 & 2.20677900 & C & 5.13211700 & 0.13880800 & -1.22353400 \\
\hline C & 4.51483300 & 2.90486400 & -1.25694800 & $\mathrm{C}$ & 5.91490200 & 1.28946800 & -1.21795000 \\
\hline C & 3.91559700 & 2.76489600 & -2.66362900 & $\mathrm{C}$ & 6.41240600 & 1.81618000 & 1700 \\
\hline $\mathrm{H}$ & -3.59610700 & 3.60940200 & 1.84413700 & C & 6.11587500 & 1.15967500 & 1.17553500 \\
\hline $\mathrm{H}$ & -3.78577700 & 1.84995800 & 1.64780900 & C & 5.33540000 & 0.01073600 & 1.18984200 \\
\hline $\mathrm{H}$ & -1.23527300 & 3.80488500 & -0.67341600 & $\mathrm{C}$ & 7.26628700 & 3.05784000 & -0.01 \\
\hline $\mathrm{H}$ & -3.01895600 & 2.96906400 & -2.09998500 & $S$ & 3.78204700 & -1.91857900 & -0.00532200 \\
\hline $\mathrm{H}$ & -2.98543500 & 1.38270400 & -1.27441300 & $\mathrm{O}$ & 4.01846500 & -2.62267400 & 1.24392600 \\
\hline $\mathrm{H}$ & -1.20907800 & 1.96283100 & 2.91996700 & $\mathrm{O}$ & 3.90525800 & -2.55942200 & -1.30343300 \\
\hline $\mathrm{H}$ & 1.03781700 & 1.32521900 & 2.04982800 & o & -2.40608600 & 0.19634800 & 8900 \\
\hline $\mathrm{H}$ & 0.99745700 & 3.05254900 & 1.78227300 & $\mathrm{Si}$ & -4.05988500 & 0.09999700 & 0.07545700 \\
\hline $\mathrm{H}$ & -0.87092200 & 0.78786300 & -0.70498100 & $\mathrm{C}$ & -4.80918400 & 1.85064800 & 0.06795000 \\
\hline $\mathrm{H}$ & 0.74632100 & 5600 & -2.60750500 & $\mathrm{C}$ & -5.06661700 & 36172700 & -1.35706000 \\
\hline $\mathrm{H}$ & -0.89541200 & 1.68626900 & -3.00803200 & - & -6.09375600 & 1.95075900 & 0.90371000 \\
\hline $\mathrm{H}$ & 0.33812000 & 2.90022600 & -2.58649400 & $\mathrm{C}$ & -4.74600500 & -0.92239200 & -1.36100900 \\
\hline $\mathrm{H}$ & -5.16944900 & 0.39288500 & -2.06537600 & C & -6.22878300 & -1.27196200 & -1.17813400 \\
\hline $\mathrm{H}$ & -5.42005500 & -2.00709600 & -1.49720000 & C & -3.92221900 & -2.18215400 & -1.66520700 \\
\hline $\mathrm{H}$ & -6.68662900 & -0.94602700 & 2.46224600 & $\mathrm{C}$ & -4.32390900 & -0.70235200 & 1.76964100 \\
\hline $\mathrm{H}$ & -6.42197700 & 1.46598200 & 1.89846500 & $\mathrm{C}$ & -3.90241600 & -2.17644100 & 1.80828300 \\
\hline H & -6.96006400 & -3.23330700 & 1.66519600 & C & -3.56121000 & 0.08374300 & 2.84519300 \\
\hline $\mathrm{H}$ & -6.47383400 & -3.70057400 & 0.02368700 & $\mathrm{H}$ & 1.54480000 & -1.47602500 & 2.06354000 \\
\hline $\mathrm{H}$ & -5.25504400 & -3.50183600 & 1.28020300 & $\mathrm{H}$ & 2.57222500 & -0.05459400 & 1.76734900 \\
\hline $\mathrm{H}$ & 2.28430500 & 5.18096600 & -1.11025200 & $\mathrm{H}$ & -0.35384800 & -1.13015200 & -0.56919200 \\
\hline $\mathrm{H}$ & 3.11306800 & 7.04558400 & 0.34647100 & $\mathrm{H}$ & 1.64761500 & -1.32093900 & -1.93893800 \\
\hline $\mathrm{H}$ & 4.47121000 & 6.00377900 & -0.07493600 & $\mathrm{H}$ & 2.25470100 & 0.25731700 & -1.34863800 \\
\hline $\mathrm{H}$ & 3.73263500 & 5.88897600 & 1.52697000 & $\mathrm{H}$ & 0.31461300 & 1.35389500 & 2.55428600 \\
\hline $\mathrm{H}$ & 3.10861900 & 3.34874400 & 2.51812600 & $\mathrm{H}$ & -0.73616600 & 2.94309500 & 0.75464800 \\
\hline $\mathrm{H}$ & 4.45199500 & 1.37224600 & 3.23409100 & $\mathrm{H}$ & -1.94804800 & 2.01205200 & 1.61808400 \\
\hline H. & 3.17642300 & 0.81454800 & 2.14259900 & $\mathrm{H}$ & -2.17334400 & 2.11117600 & -0.93178500 \\
\hline 11 & 4.82384900 & 0.96518200 & 1.55237500 & $\mathrm{H}$ & 0.27523000 & 1.75068200 & -1.34508500 \\
\hline $\mathrm{H}$ & 5.22512700 & 3.74320500 & -1.23159700 & $\mathrm{H}$ & -1.35765500 & 1.35683900 & -3.18425700 \\
\hline $\mathrm{H}$ & 4.69833700 & 2.58214000 & -3.40372100 & $\mathrm{H}$ & -0.03989300 & 0.18111900 & -3.30164700 \\
\hline 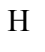 & 3.37249500 & 3.66796400 & -2.96350200 & $\mathrm{H}$ & -1.60611300 & -0.30689100 & -2.62557500 \\
\hline $\mathrm{H}$ & 3.21233100 & 1.92766500 & -2.70778300 & $\mathrm{H}$ & 4.76851700 & -0.29025700 & -2.15231000 \\
\hline
\end{tabular}




\begin{tabular}{|c|c|c|c|c|c|c|c|}
\hline B & 1.12493600 & -1.20778500 & 0.02233500 & $\mathrm{H}$ & 6.15170800 & 1.78116900 & -2.15754600 \\
\hline $\mathrm{C}$ & 1.83346500 & -1.45896800 & 1.46582800 & $\mathrm{H}$ & 6.51163700 & 1.55131800 & 2.10926800 \\
\hline $\mathrm{C}$ & 1.16626300 & -1.04970400 & 2.61979500 & $\mathrm{H}$ & 5.12677800 & -0.52004900 & 2.11362400 \\
\hline $\mathrm{C}$ & 3.12246700 & -1.93272200 & 1.68871100 & $\mathrm{H}$ & 8.25981500 & 2.84553100 & 0.39184700 \\
\hline $\mathrm{C}$ & 1.71509300 & -1.06303700 & 3.89175300 & $\mathrm{H}$ & 7.39324600 & 3.46079800 & -1.02354800 \\
\hline $\mathrm{C}$ & 3.71639400 & -1.97233500 & 2.94790000 & $\mathrm{H}$ & 6.81734400 & 3.83739800 & 0.60780800 \\
\hline $\mathrm{C}$ & 3.01342600 & -1.52854100 & 4.05633500 & $\mathrm{H}$ & -4.06137700 & 2.51164000 & 0.53385800 \\
\hline $\mathrm{C}$ & 2.10920900 & -1.41521500 & -1.25846700 & $\mathrm{H}$ & -5.38894800 & 3.40981200 & -1.34681500 \\
\hline $\mathrm{C}$ & 1.93639800 & -2.31164900 & -2.30929600 & $\mathrm{H}$ & -4.17946900 & 2.29102000 & -1.99785700 \\
\hline $\mathrm{C}$ & 3.25026600 & -0.62790100 & -1.36772300 & $\mathrm{H}$ & -5.86192500 & 1.78100000 & -1.83932600 \\
\hline $\mathrm{C}$ & 2.80929800 & -2.40609000 & -3.39025100 & $\mathrm{H}$ & -6.51137200 & 2.96381900 & 0.85325000 \\
\hline $\mathrm{C}$ & 4.15357300 & -0.68969100 & -2.41468000 & $\mathrm{H}$ & -5.91964900 & 1.71528800 & 1.95824400 \\
\hline $\mathrm{C}$ & 3.92375400 & -1.58594700 & -3.44916000 & $\mathrm{H}$ & -6.86623500 & 1.26460700 & 0.53680400 \\
\hline $\mathrm{C}$ & -0.34320700 & -1.89893300 & -0.15808100 & $\mathrm{H}$ & -4.65811300 & -0.26010100 & -2.23670500 \\
\hline $\mathrm{C}$ & -1.10461100 & -1.65399900 & -1.30126600 & $\mathrm{H}$ & -6.60383400 & -1.85349100 & -2.02920600 \\
\hline $\mathrm{C}$ & -0.92916400 & -2.80437500 & 0.72623000 & $\mathrm{H}$ & -6.85772600 & -0.37898900 & -1.08852600 \\
\hline $\mathrm{C}$ & -2.33680800 & -2.23012500 & -1.56743500 & $\mathrm{H}$ & -6.38370400 & -1.87825700 & -0.27659000 \\
\hline $\mathrm{C}$ & -2.15572600 & -3.42107400 & 0.49557600 & $\mathrm{H}$ & -4.22324100 & -2.61581200 & -2.62650000 \\
\hline C & -2.85300300 & -3.14967100 & -0.66822000 & $\mathrm{H}$ & -2.84959500 & -1.96764000 & -1.70864300 \\
\hline $\mathrm{H}$ & 0.93403200 & 0.04732500 & 0.05685100 & $\mathrm{H}$ & -4.07147000 & -2.95236700 & -0.90248800 \\
\hline $\mathrm{F}$ & 0.90181700 & -3.16194400 & -2.33398600 & $\mathrm{H}$ & -5.40000700 & -0.64492700 & 1.99377300 \\
\hline $\mathrm{F}$ & 2.58096300 & -3.28233100 & -4.36675500 & $\mathrm{H}$ & -3.94913300 & -2.56727400 & 2.83234600 \\
\hline $\mathrm{F}$ & 4.76970500 & -1.66221500 & -4.47254300 & $\mathrm{H}$ & -4.55042800 & -2.80174400 & 1.18732200 \\
\hline $\mathrm{F}$ & 5.23459100 & 0.09811400 & -2.44157400 & $\mathrm{H}$ & -2.87177800 & -2.30201700 & 1.45468700 \\
\hline $\mathrm{F}$ & 3.54853700 & 0.23537900 & -0.36959300 & $\mathrm{H}$ & -3.76275100 & -0.32178800 & 3.84398400 \\
\hline $\mathrm{F}$ & -0.09753700 & -0.58867800 & 2.51861600 & $\mathrm{H}$ & -3.83027200 & 1.14757800 & 2.85859900 \\
\hline $\mathrm{F}$ & 1.01854300 & -0.63111300 & 4.94430800 & $\mathrm{H}$ & -2.48088300 & 0.01148500 & 2.66931700 \\
\hline $\mathrm{F}$ & 3.57320900 & -1.54688200 & 5.26295000 & & & & \\
\hline $\mathrm{F}$ & 4.96073400 & -2.42607600 & 3.09291600 & & & & \\
\hline $\mathrm{F}$ & 3.88357100 & -2.37912500 & 0.68115800 & & & & \\
\hline $\mathrm{F}$ & -0.31769900 & -3.17055200 & 1.85939200 & & & & \\
\hline $\mathrm{F}$ & -2.66111300 & -4.28499300 & 1.37837100 & & & & \\
\hline $\mathrm{F}$ & -4.00474100 & -3.77179300 & -0.92588000 & & & & \\
\hline $\mathrm{F}$ & -3.01284700 & -1.93454400 & -2.68098200 & & & & \\
\hline $\mathrm{F}$ & -0.62258400 & -0.84081000 & -2.26151900 & & & & \\
\hline $\mathrm{H}$ & 1.59059700 & 5.03992400 & 0.49469400 & & & & \\
\hline $\mathrm{H}$ & 4.73322800 & 3.46564600 & 1.89488500 & & & & \\
\hline $\mathrm{H}$ & 5.08394500 & 2.00643300 & -1.00365300 & & & & \\
\hline
\end{tabular}




\section{References}

[1] Massey, A. G.; Park, A. J. J. Organomet. Chem. 1964, 2, 245.

[2] Choony, N.; Kuhnert, N.; Sammes, P. G.; Smith, G.; Ward, R. W. J. Chem. Soc., Perkin Trans. 1, 2002, 1999.

[3] Naimi-Jamal, M. R.; Mokhtari, J.; Dekamin, M. G.; Kaupp, G. Eur. J. Org. Chem. 2009, 3567.

[4] Kennedy, J. P.; Lindsley, C. W. Tetrahedron Lett. 2010, 51, 2493.

[5] Bender, A. M.; Clark, M. J.; Agius, M. P.; Traynor, J. R.; Mosberg, H. I. Bioorg. Med. Chem. Lett. 2014, 24, 548.

[6] Dakas, P.-Y.; Jogireddy, R.; Valot, G.; Barluenga, S.; Winssinger, N. Chem. Eur. J. 2009, 15, 11490.

[7] Das, P.; McNulty, J. Tetrahedron Lett. 2010, 51, 3197.

[8] Huo, X.; He, R.; Fu, J.; Zhang, J.; Yang, G.; Zhang, W. J. Am. Chem. Soc. 2017, 139, 9819.

[9] Singh, C.; Verma, V. P.; Naikade, N. K.; Singh, A. S.; Hassam, M.; Puri, S. K. Bioorg. Med. Chem. Lett. 2010, 20, 4459.

[10]Choony, N.; Dadabhoy, A.; G. Sammes, P. J. Chem. Soc., Perkin Trans. 1, 1998, 2017.

[11] Knight, D. W.; Nott, A. P. J. Chem. Soc., Perkin Trans. 1, 1981, 1125.

[12] Arai, S.; Koike, Y.; Hada, H.; Nishida, A. J. Org. Chem. 2010, 75, 7573.

[13] Kuhnert, N.; Sammes, P. G.; Smith, G.; Ward, R. W. J. Chem. Res. 2004, 608.

[14] Gaussian 09, R. D., Frisch, M. J.; Trucks, G. W.; Schlegel, H. B.; Scuseria, G. E.; Robb, M. A.; Cheeseman, J. R.; Scalmani, G.; Barone, V.; Mennucci, B.; Petersson, G. A.; Nakatsuji, H.; Caricato, M.;Li, X.; Hratchian, H. P.; Izmaylov, A. F.; Bloino, J.; Zheng, G.; Sonnenberg, J. L.; Hada, M.; Ehara, M.; Toyota, K.; Fukuda, R.; Hasegawa, J.; Ishida, M.; Nakajima, T.; Honda, Y.; Kitao, O.; Nakai, H.; Vreven, T.; Montgomery, J. A., Jr.; Peralta, J.E.; Ogliaro, F.; Bearpark, M.; Heyd, J. J.; Brothers, E.; Kudin, K. N.; Staroverov, V. N.; Kobayashi, R.; Normand, J.; Raghavachari, K.; Rendell, A.; Burant, J. C.; Iyengar, S. S.; Tomasi, J.; Cossi, M.; Rega, N.; Millam, J. M.; Klene, M.; Knox, J. E.; Cross, J. B.; Bakken,V.; Adamo, C.; Jaramillo, J.; Gomperts, R.; Stratmann, R. E.; Yazyev, O.; Austin, A. J.; Cammi, R.; Pomelli, C.; Ochterski, J. W.; Martin, R. L.; Morokuma, K.; Zakrzewski, V. G.; Voth, G. A.; Salvador, P.; Dannenberg, J. J.; Dapprich, S.; Daniels, A. D.; Farkas, Ö.; Foresman, J. B.; Ortiz, J. V.; Cioslowski, J.; Fox, D. J.; Gaussian, Inc., Wallingford CT, 2009.

[15] Zhao, Y.; Truhlar, D. G. Theor. Chem. Acc. 2008, 120, 215.

[16] Hratchian, H. P.; Schlegel, H. B. J. Chem. Phys. 2004, 120, 9918.

[17] Hratchian, H. P.; Schlegel, H. B. J. Chem. Theory. Comput. 2005, 1, 61.

[18] Marenich, A. V.; Cramer, C. J.; Truhlar, D. G. J. Phys. Chem. B 2009, 113, 6378.

[19] Grimme, S.; Antony, J.; Ehrlich, S.; Krieg, H. J. Chem. Phys. 2010, 132, 154104.

[20] (a) Grimme, S.; Ehrlich, S.; Georigk, L. J. Comput. Chem. 2011, 32, 1456; (b) Grimme, S. Chem. Eur. J. 2012, 18, 9955. 
[21] Funes-Ardoiz, I.; Paton, R. S. GoodVibes v2.0.1; doi: 10.5281/zenodo.56091 (accessed 21 June 2016).

[22] CYLview, b. L., C. Y.; Université de Sherbrooke. 2009. 


\section{NOE Spectra}

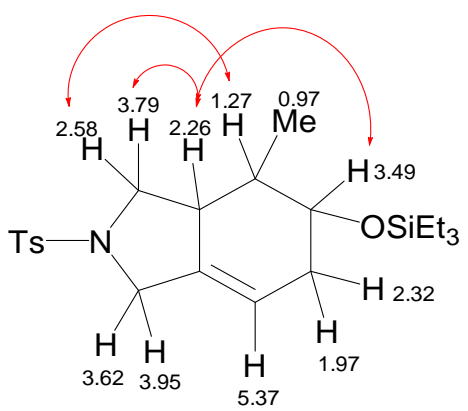

$3 \mathbf{a}$

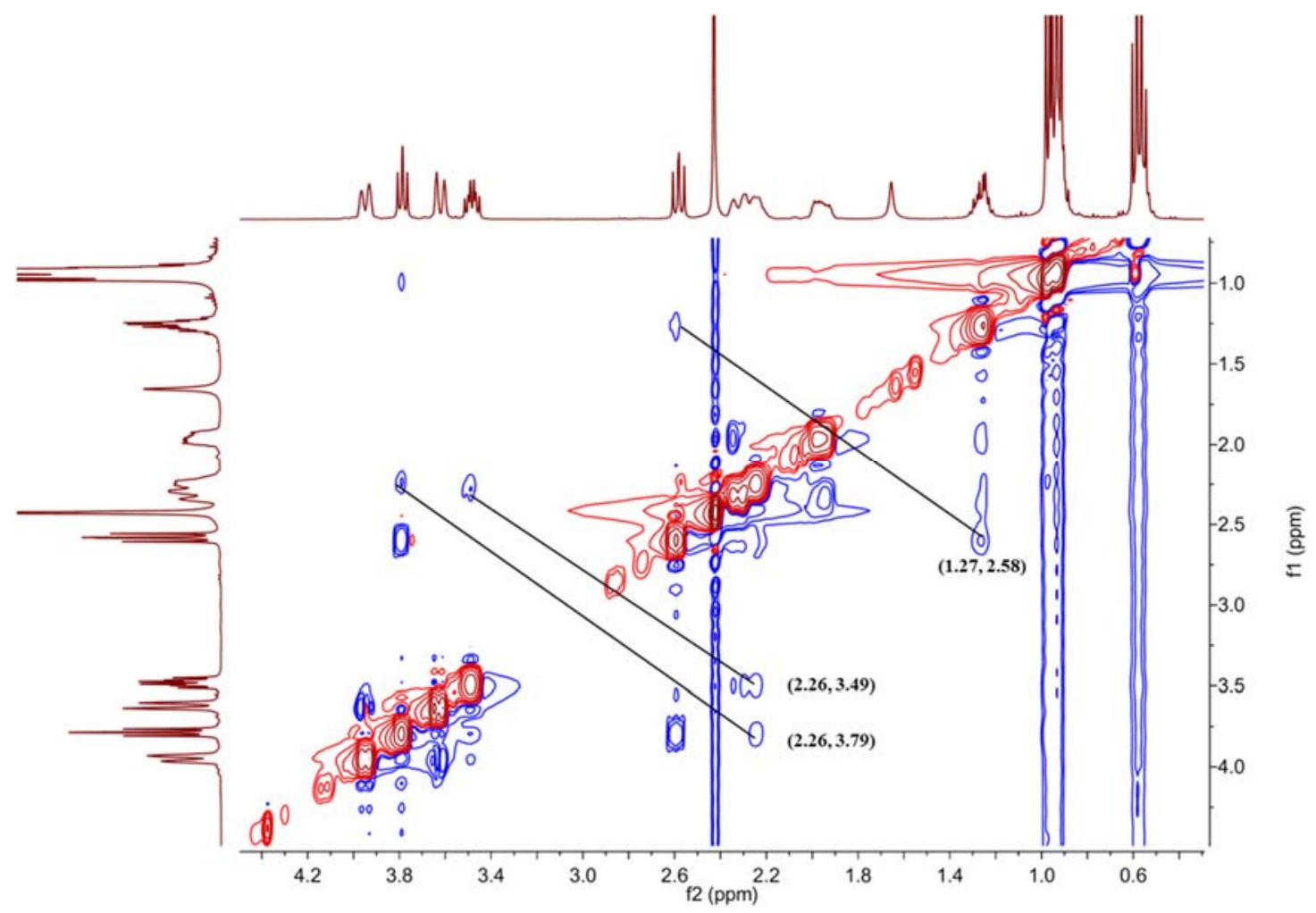




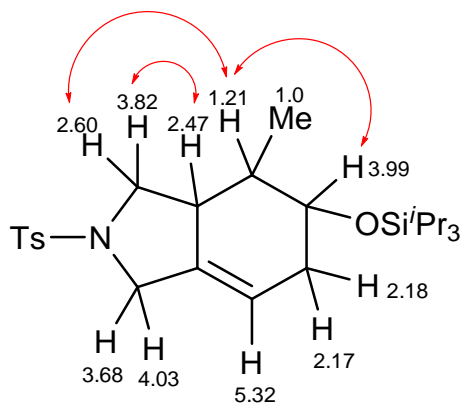

$4 a$

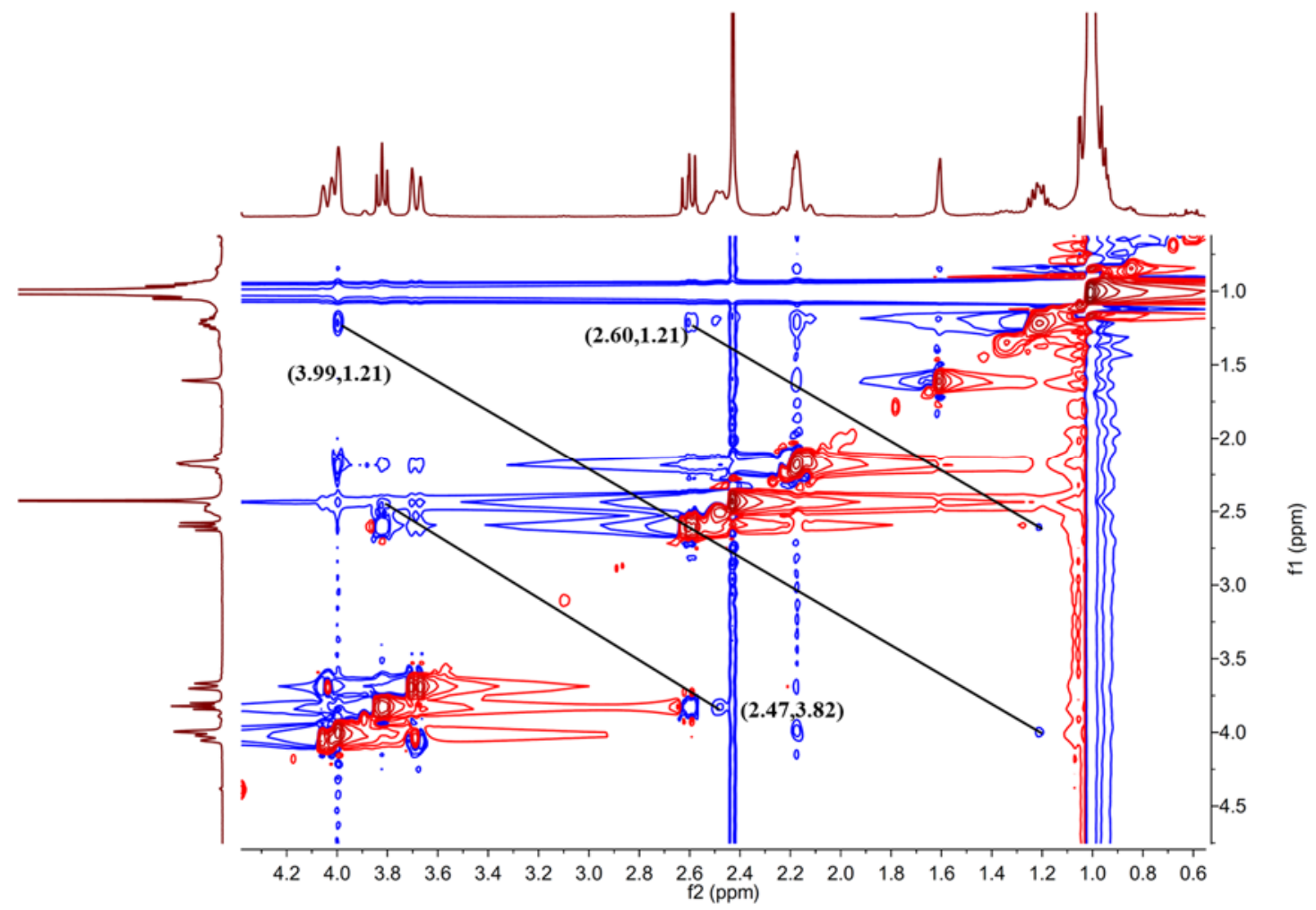




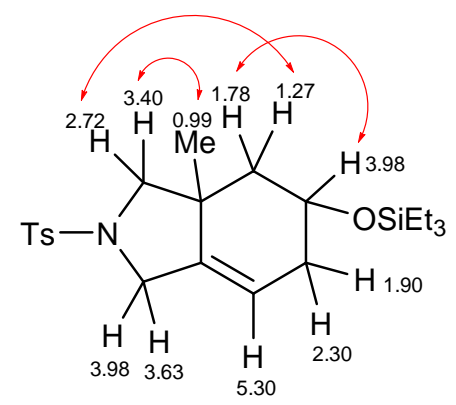

3f-major

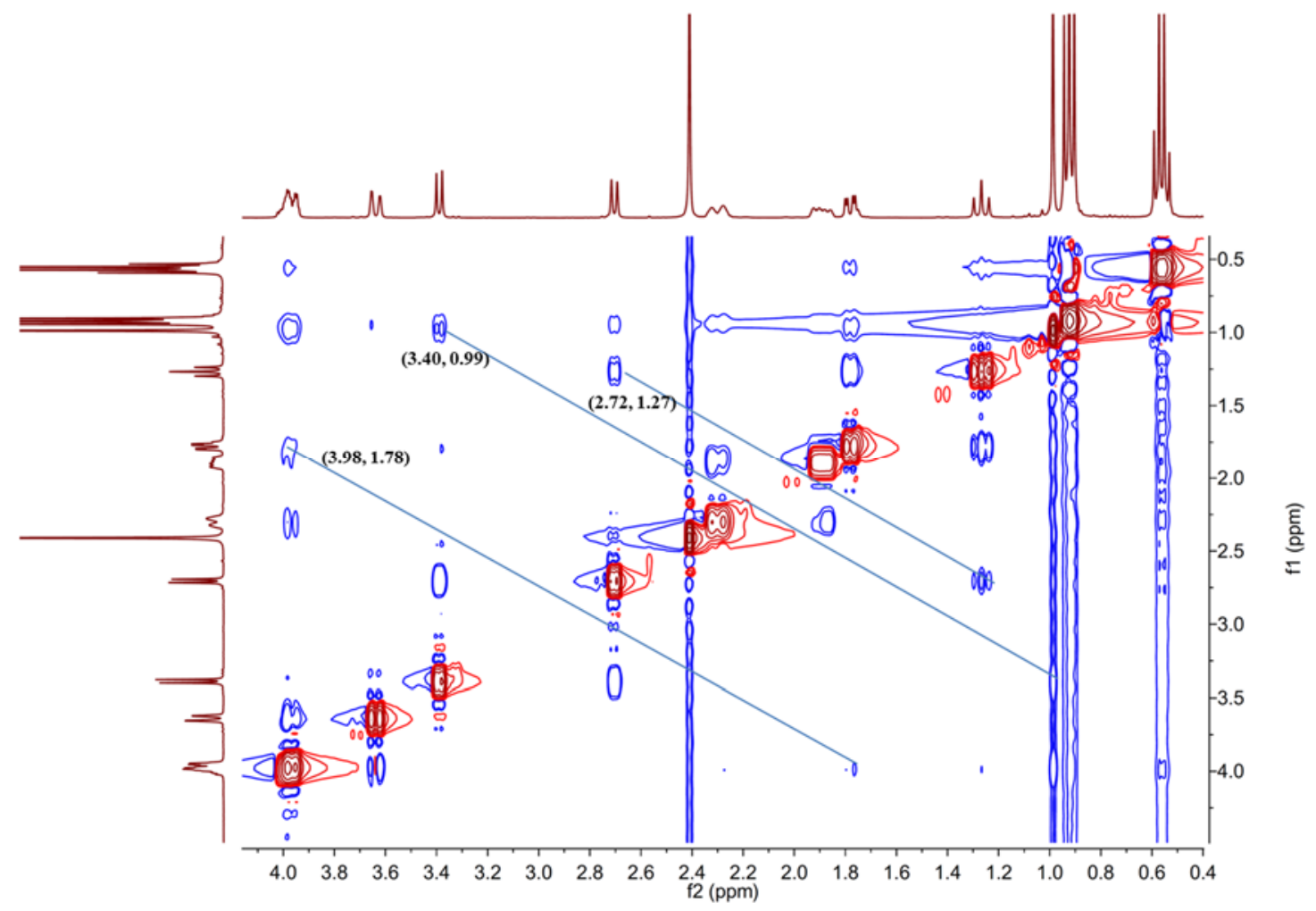




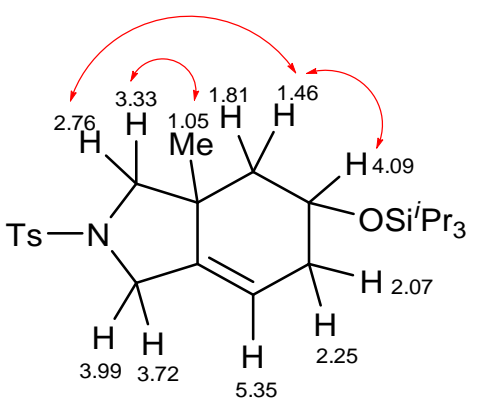

4f-major

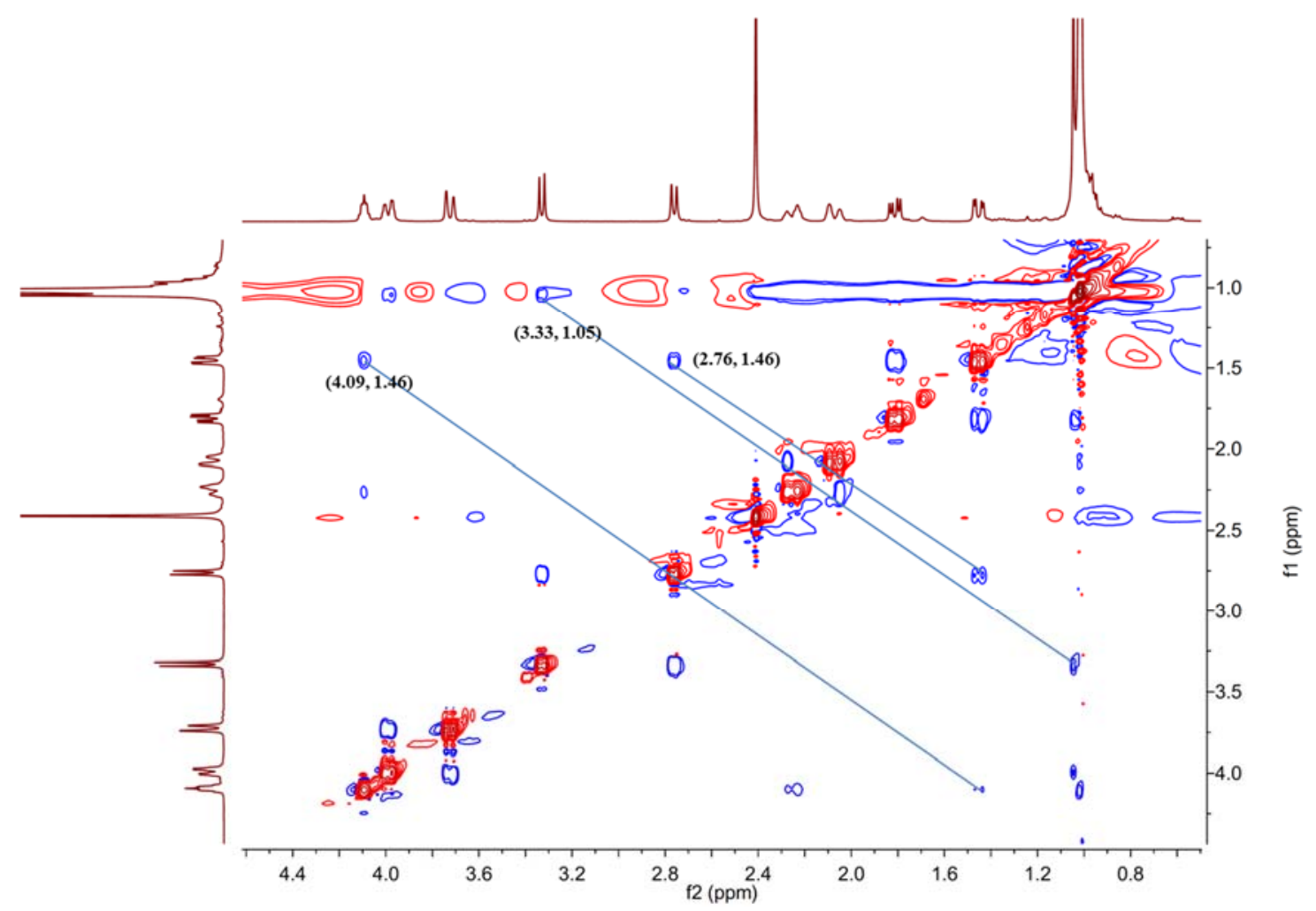




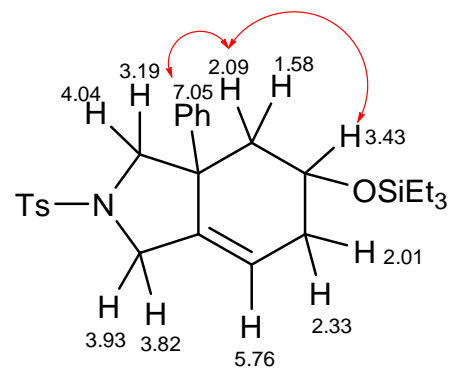

3g-major

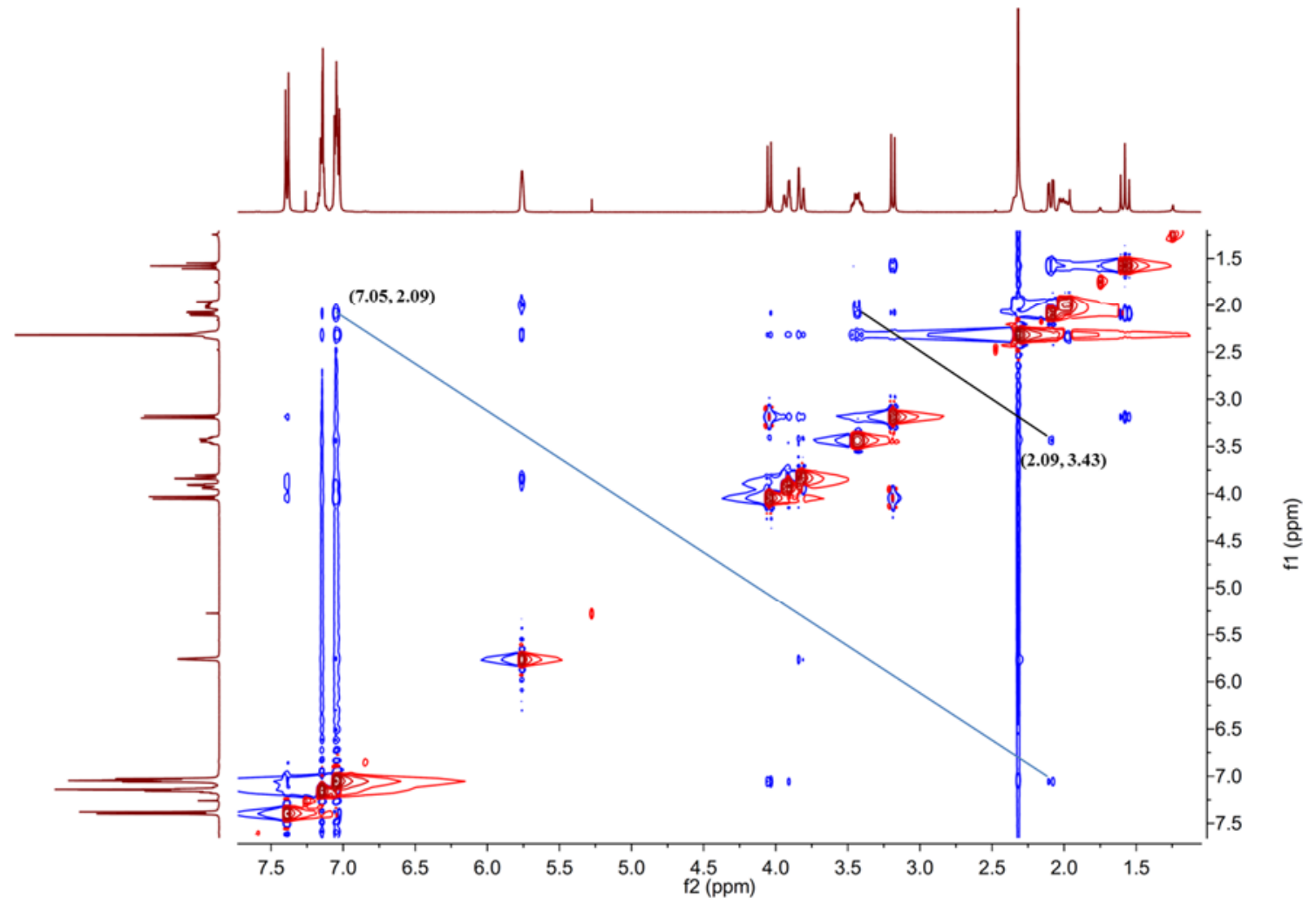




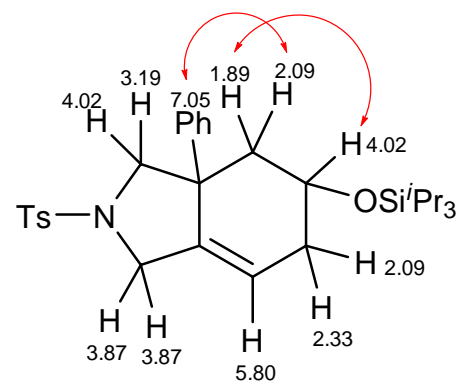

4g-major

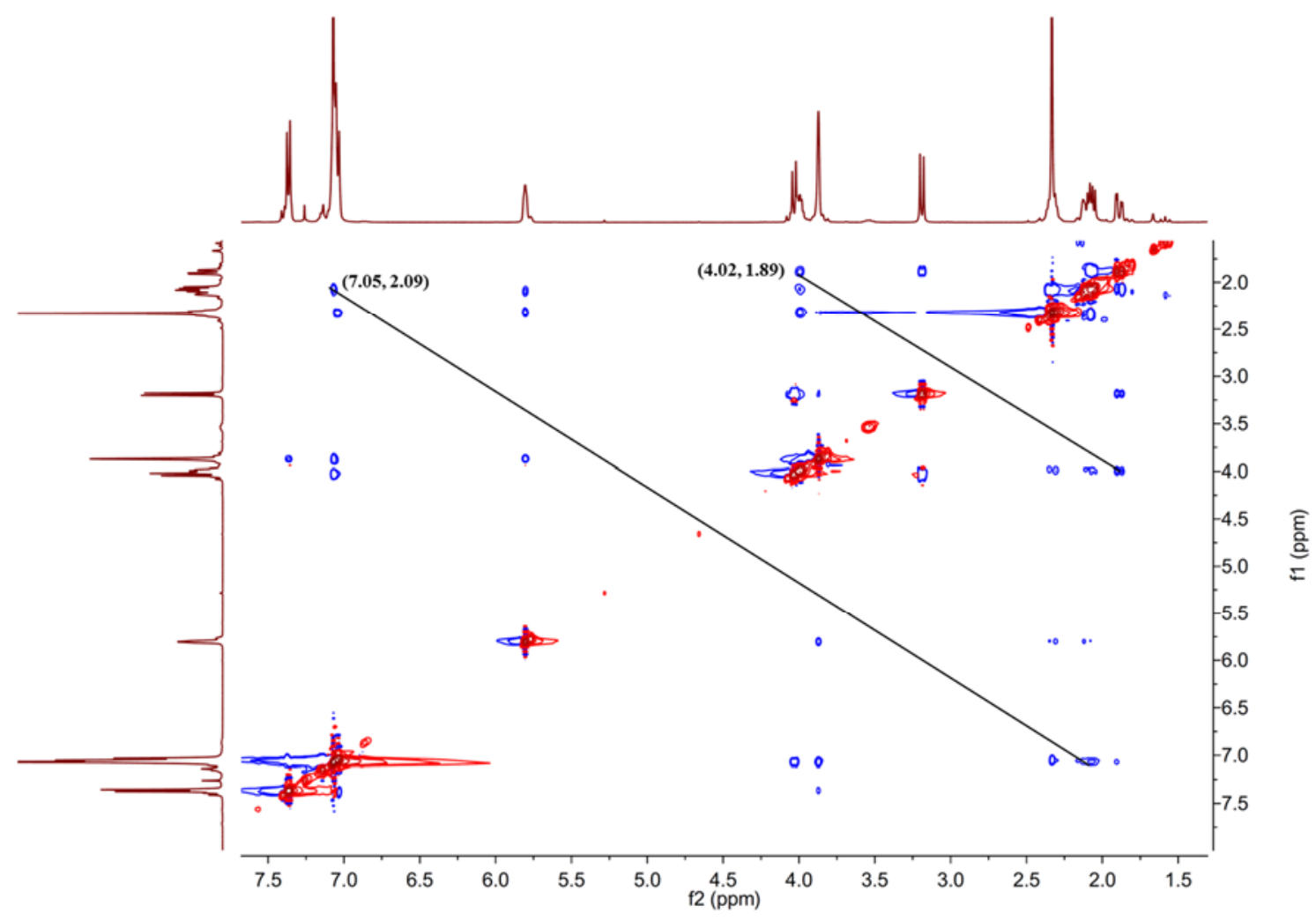



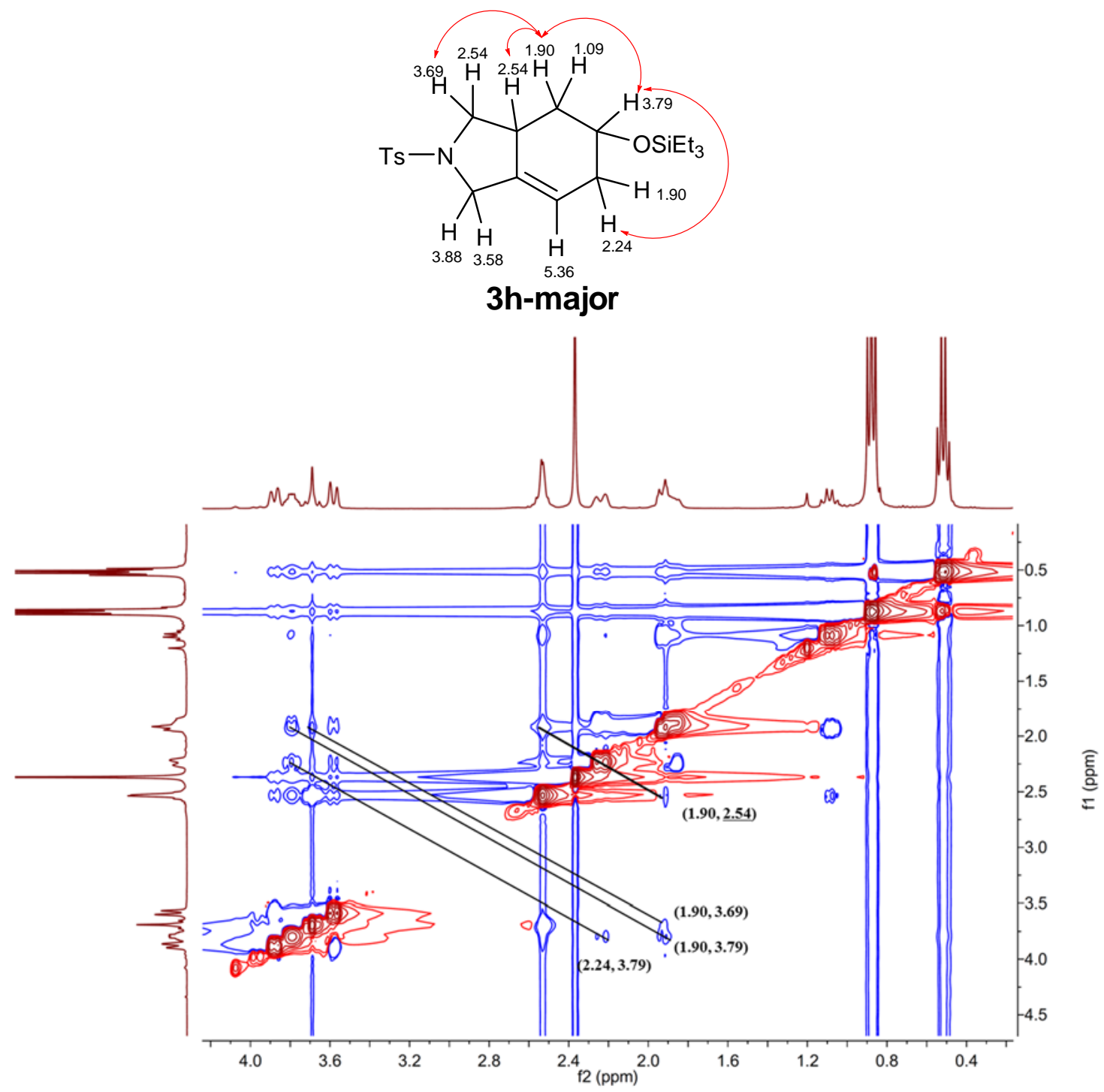


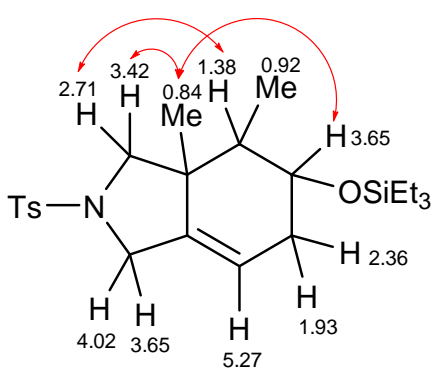

3j

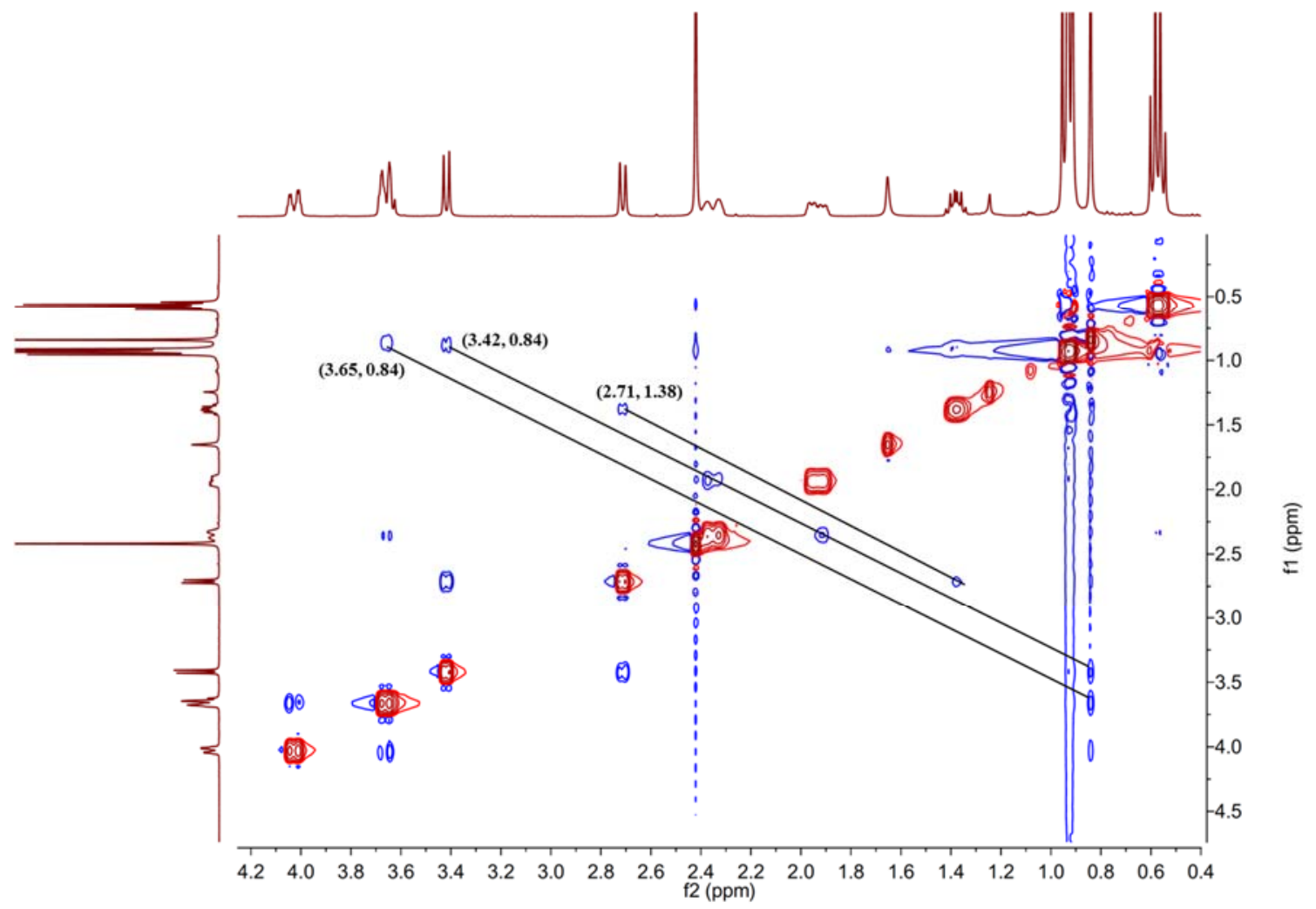




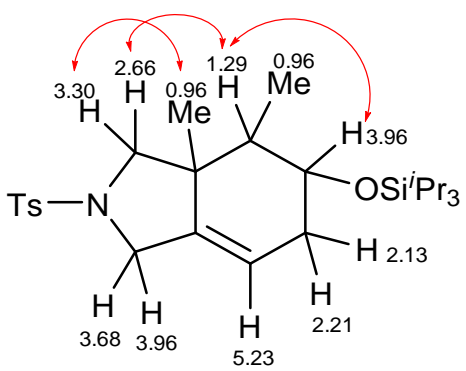

4j

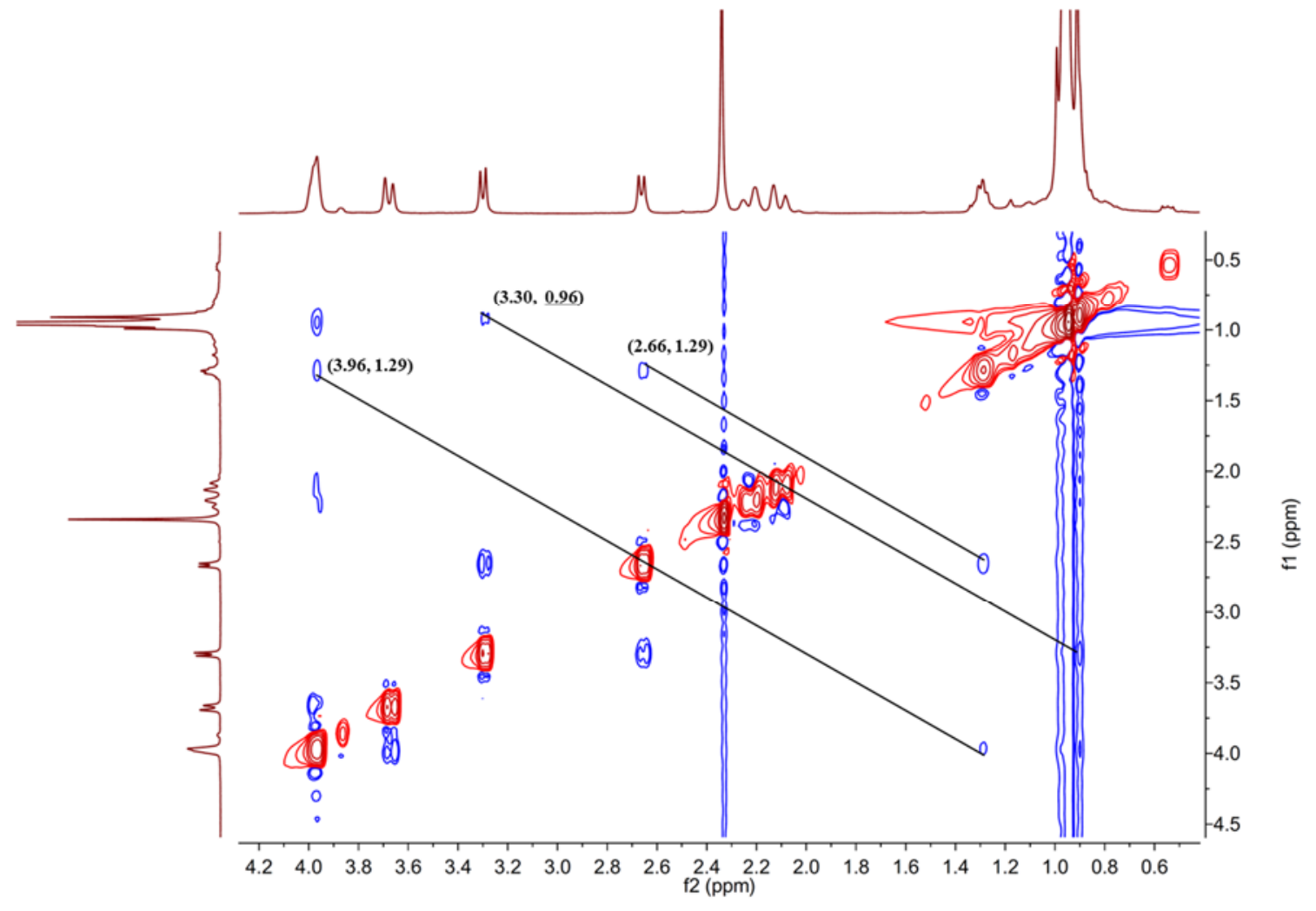




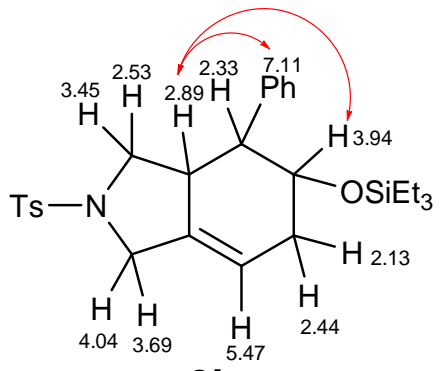

3k

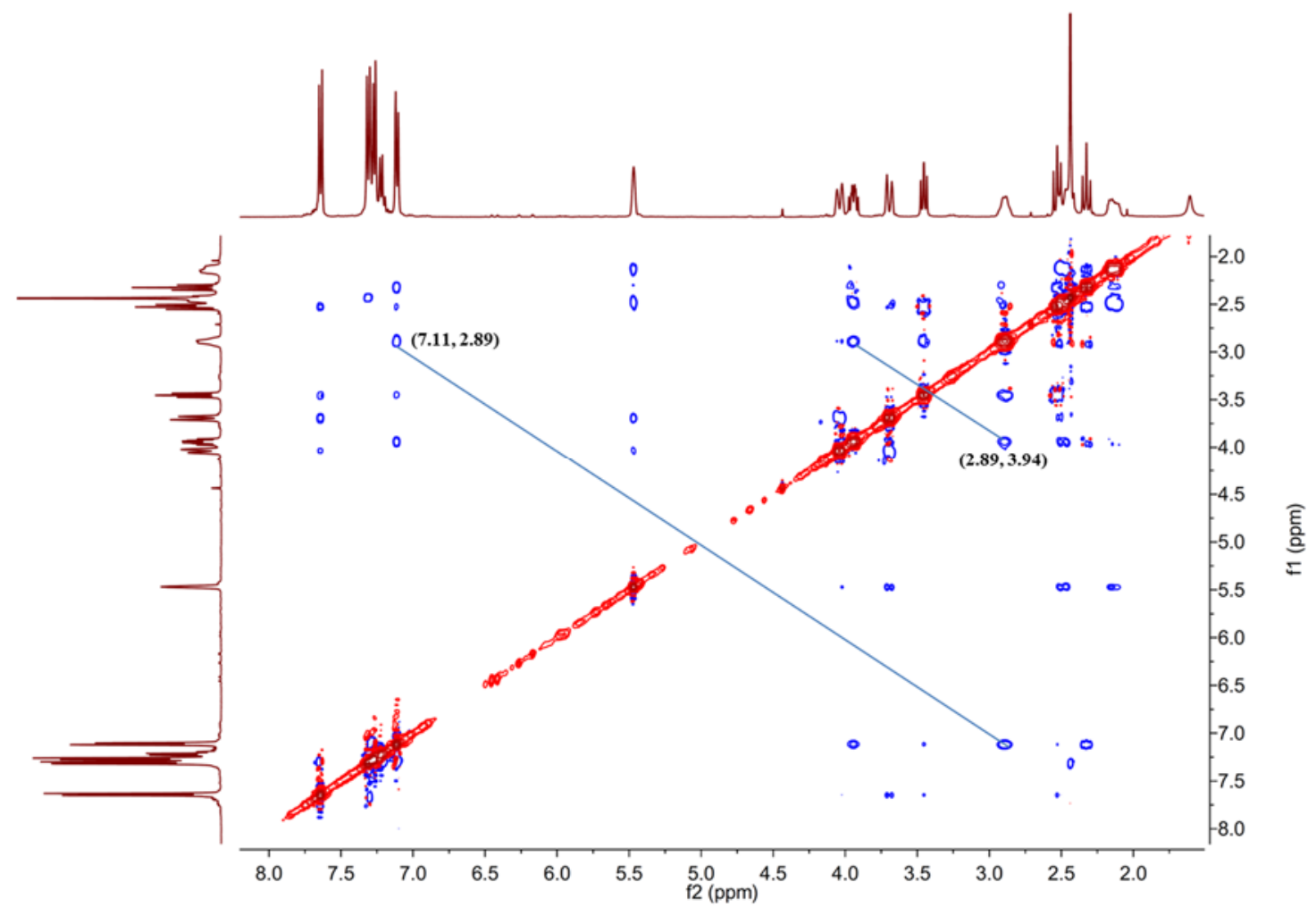



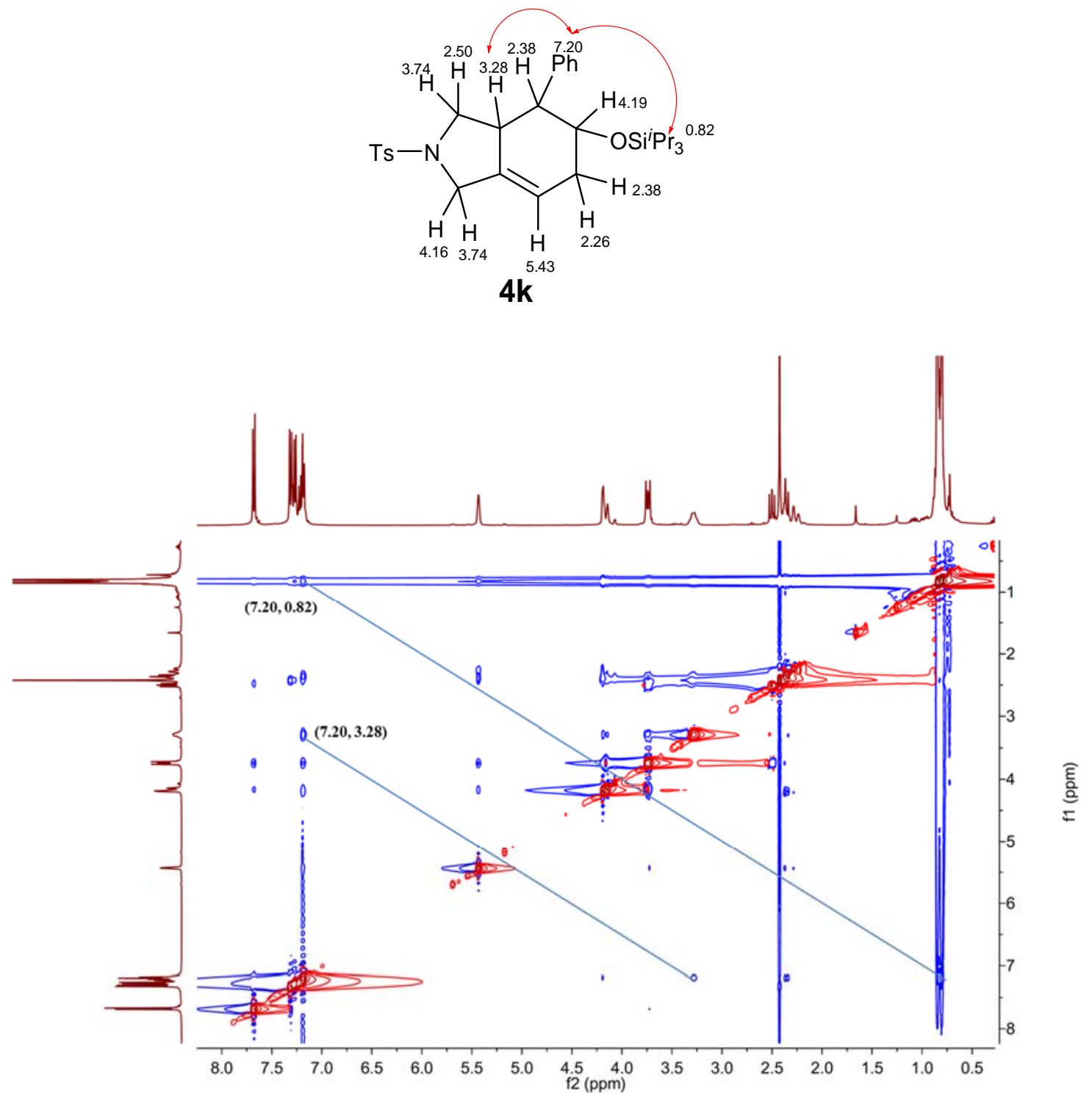


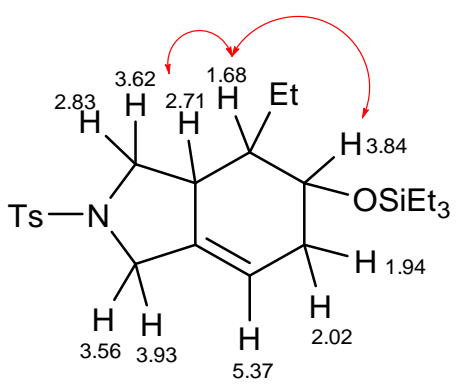

$3 q$

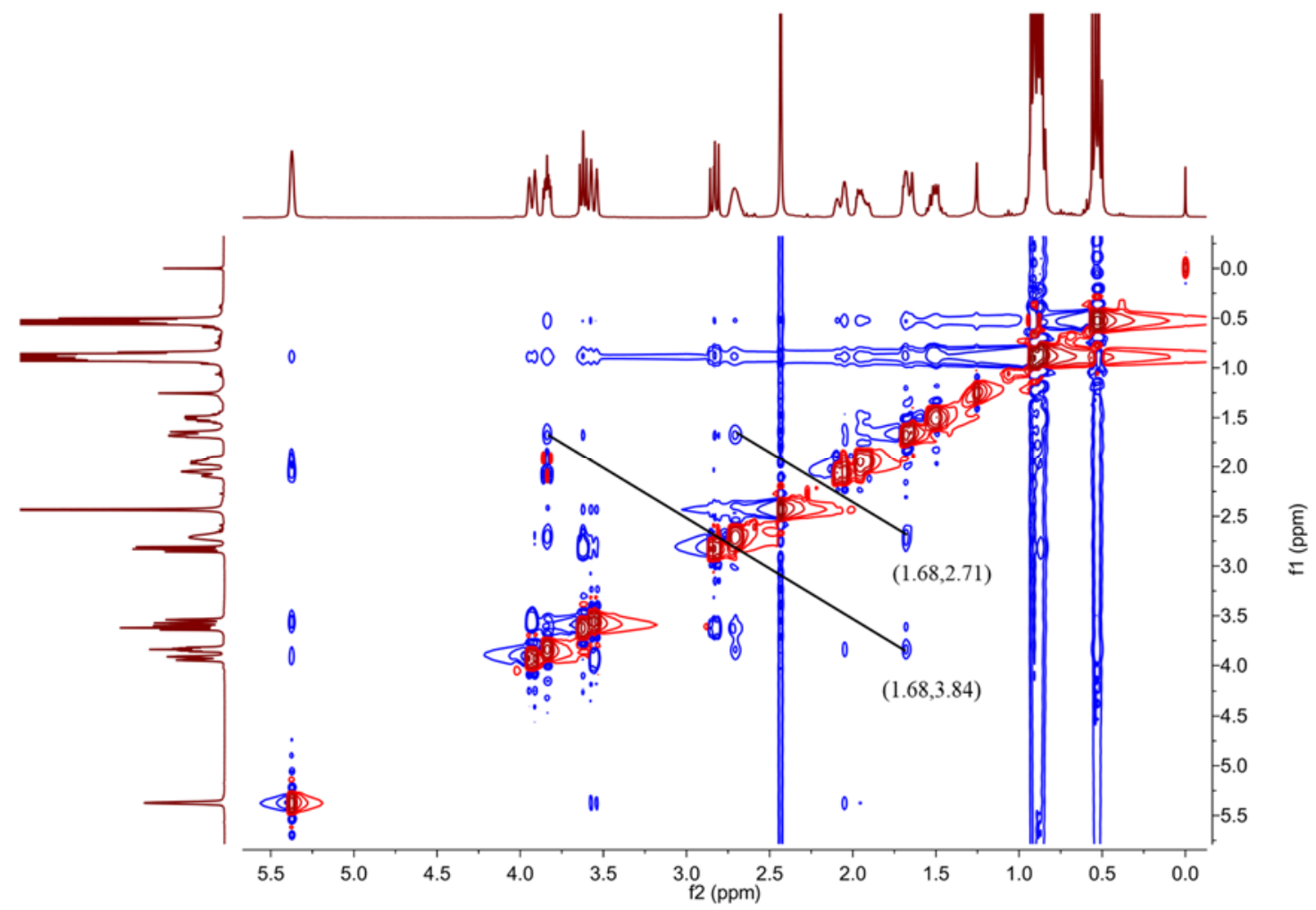




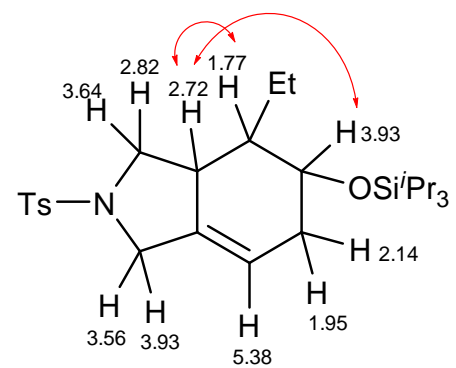

3q'-major

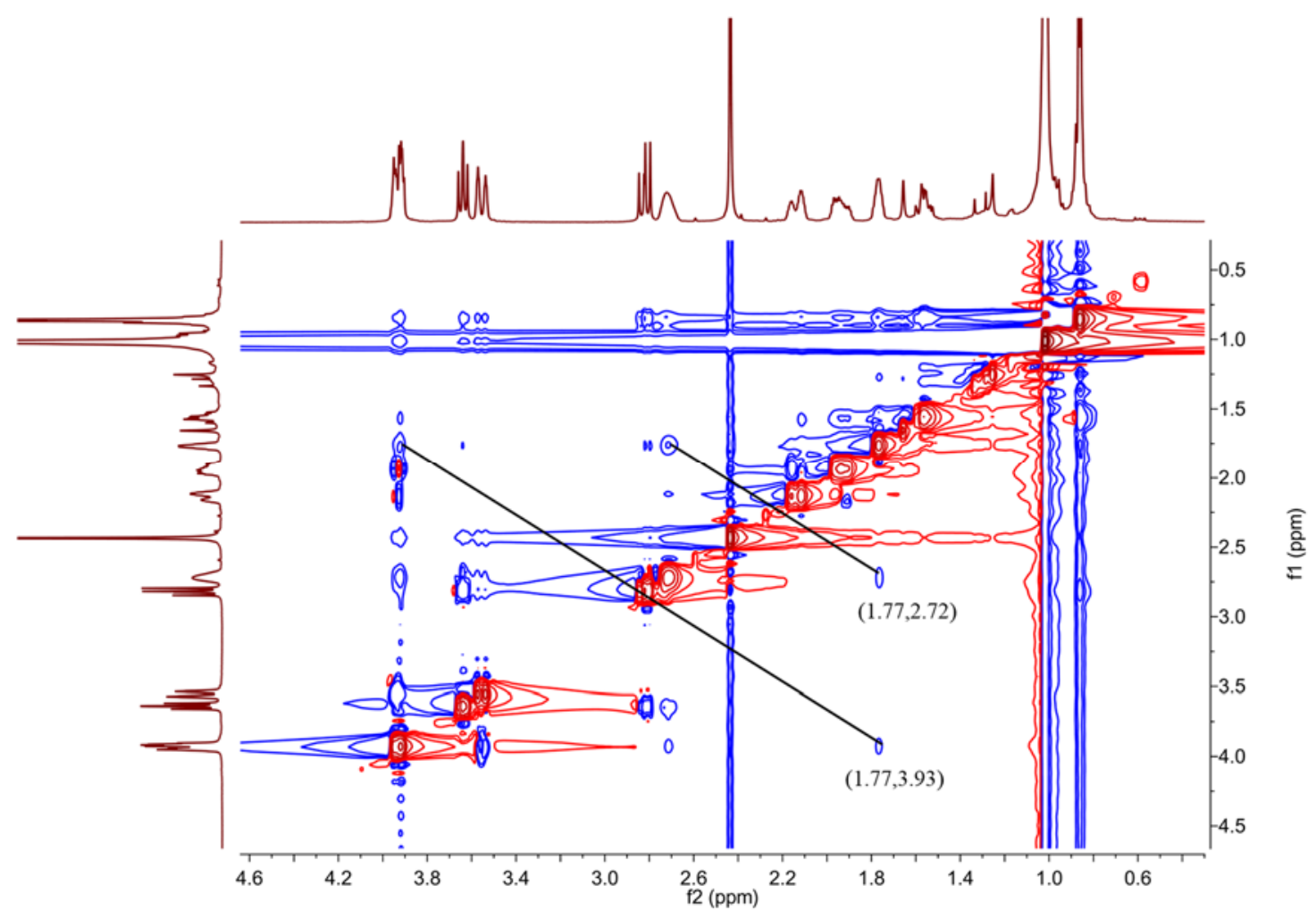



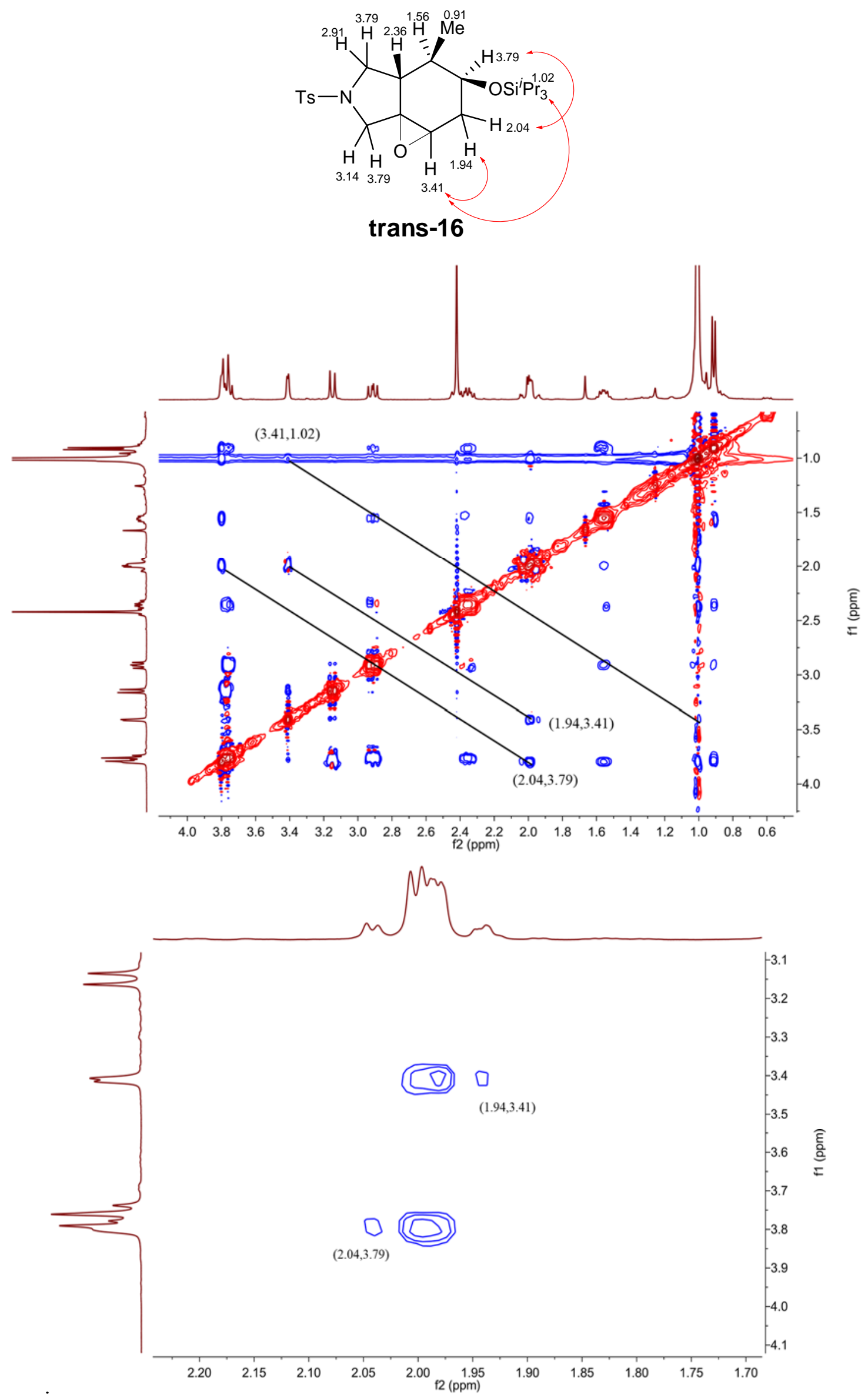

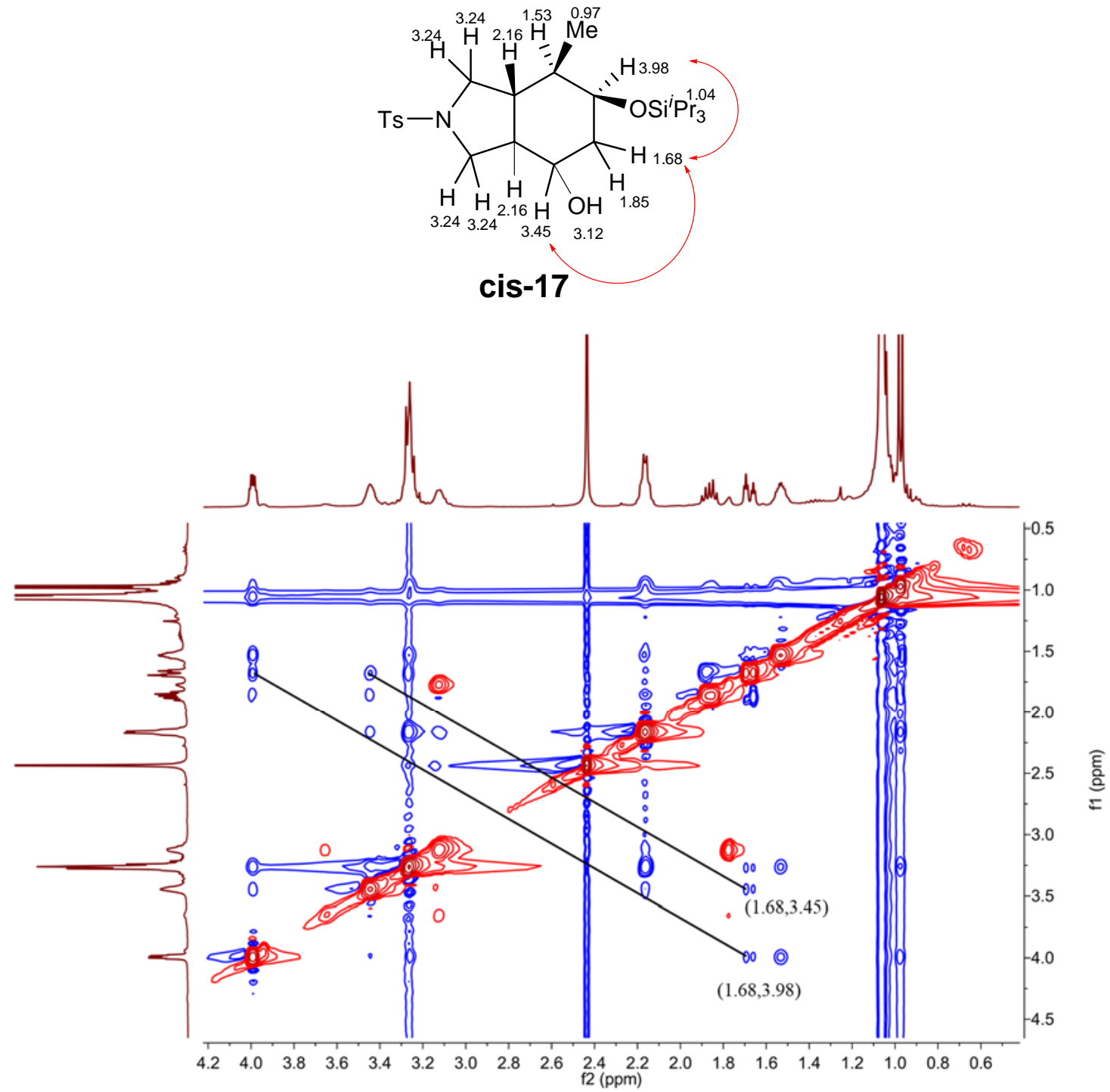

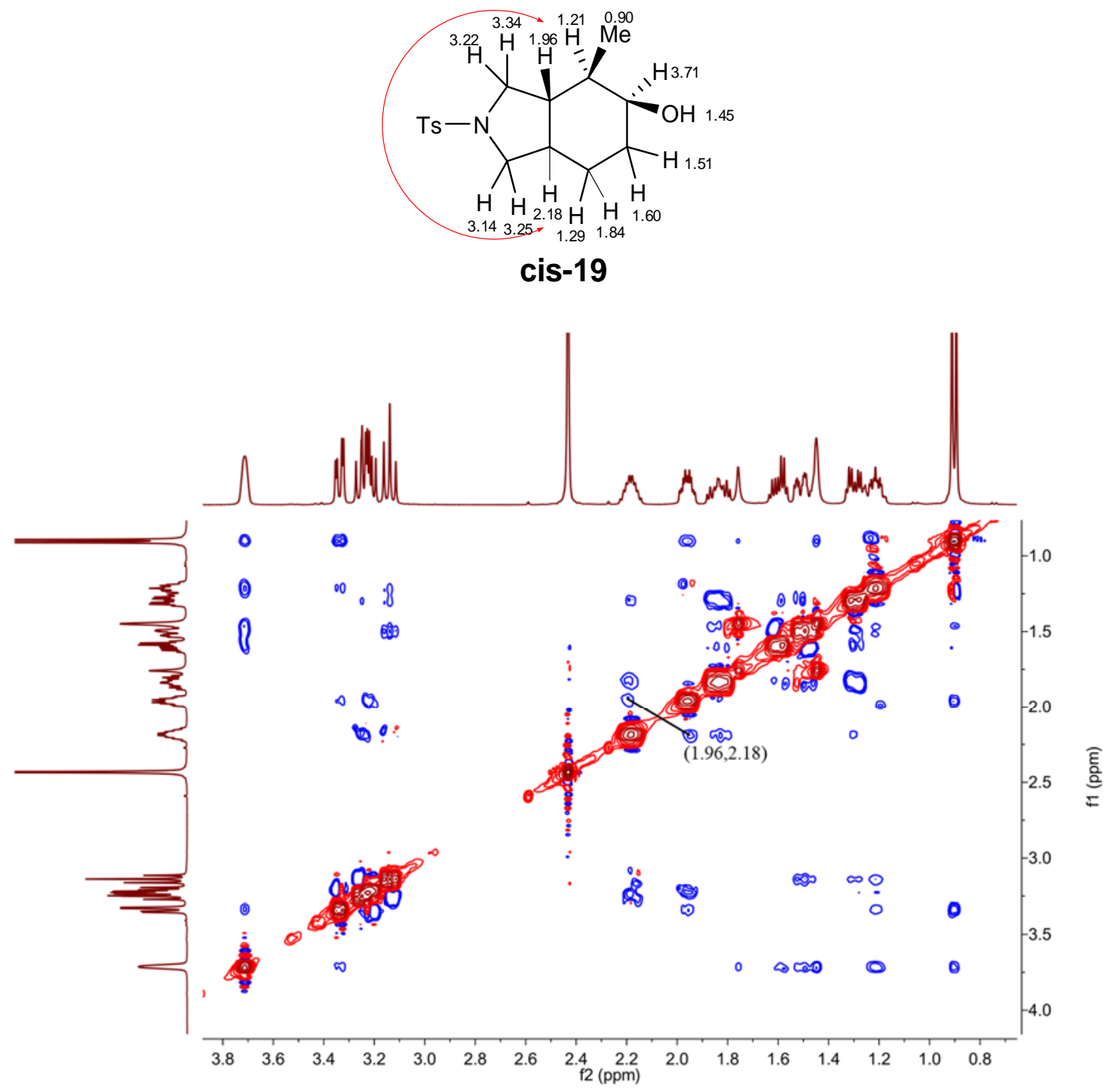


\section{NMR Spectra}

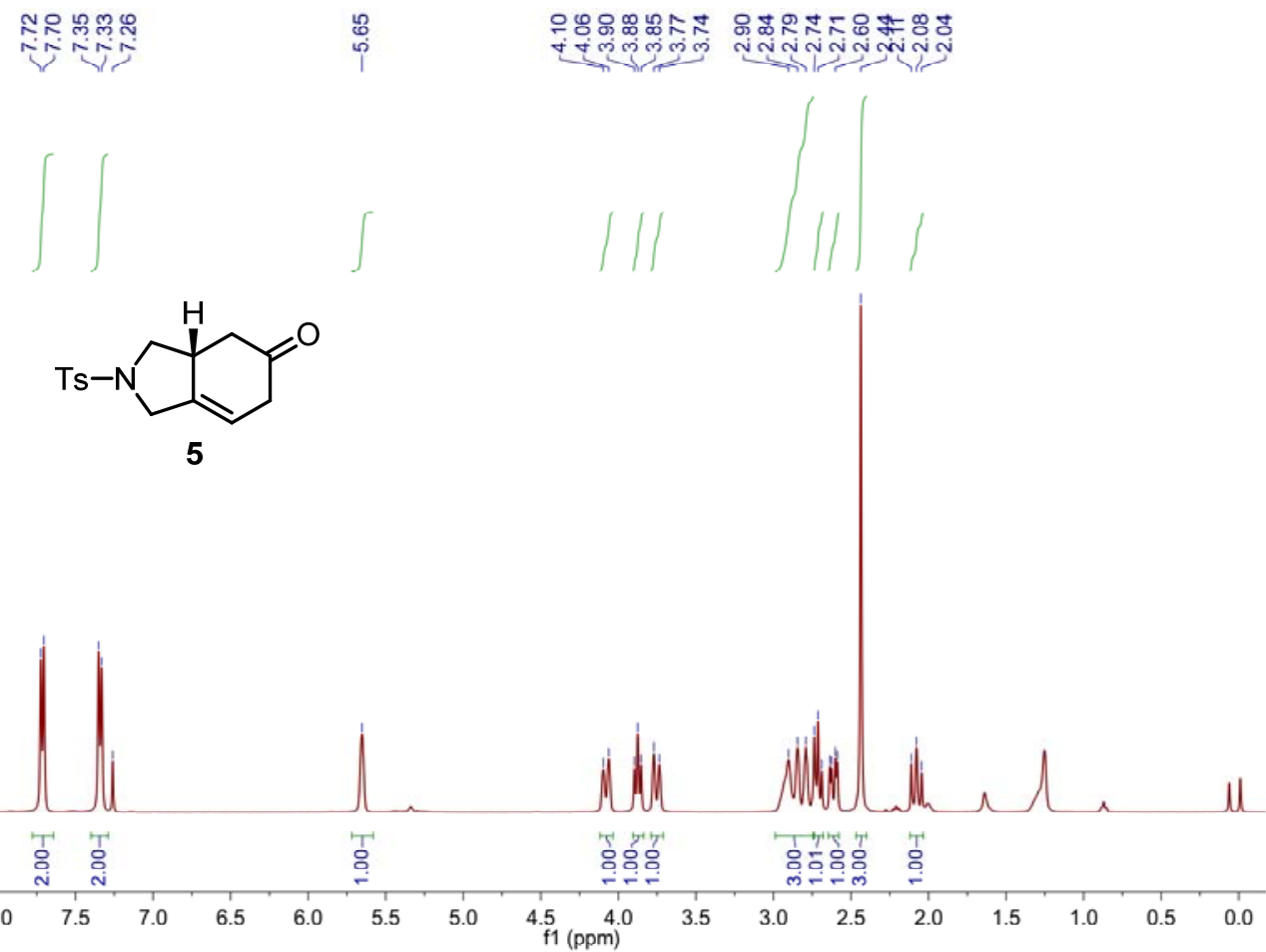

ڤั

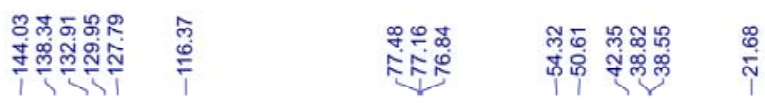<smiles>[3H]N1CC2=CCC(=O)C[C@@H]2C1</smiles>

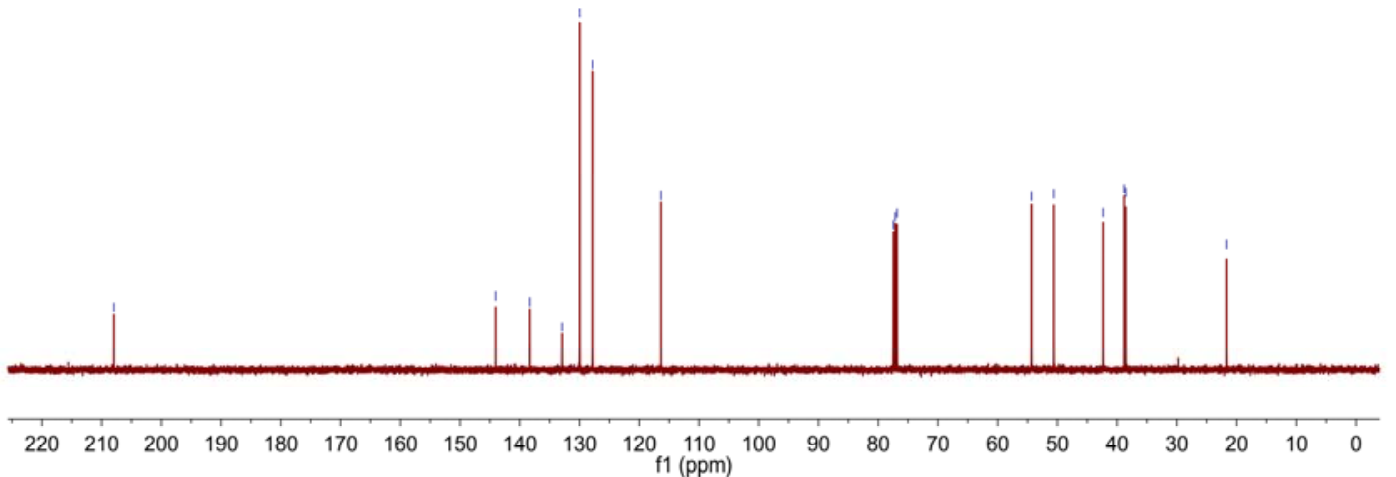




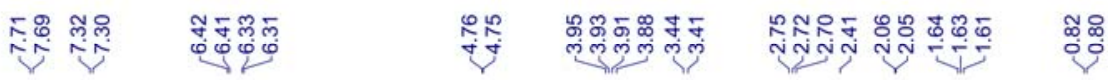

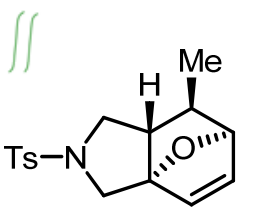

2a

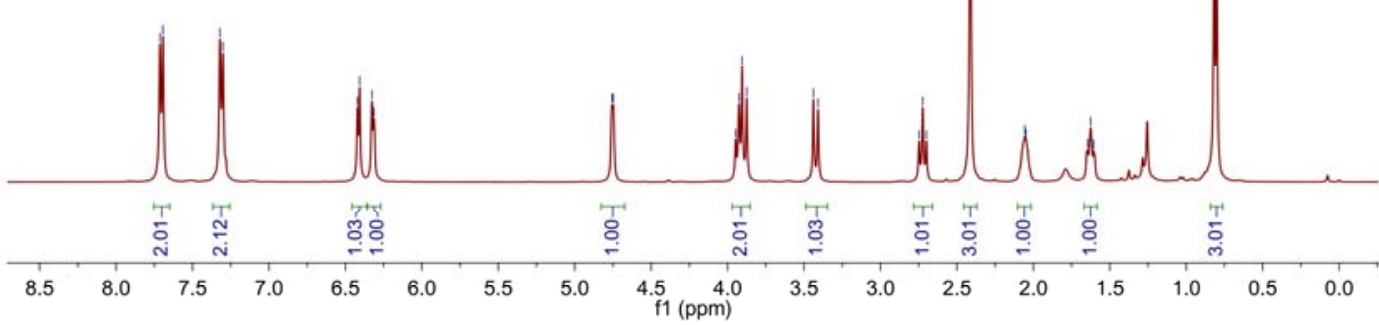

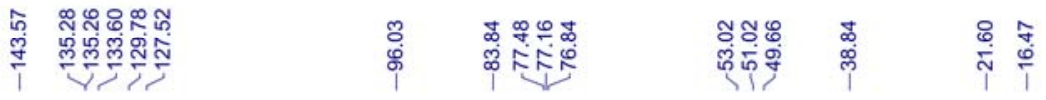<smiles>C[C@H]1[C@H]2C=C[C@@]3(CN([As])C[C@@H]13)O2</smiles>

2a

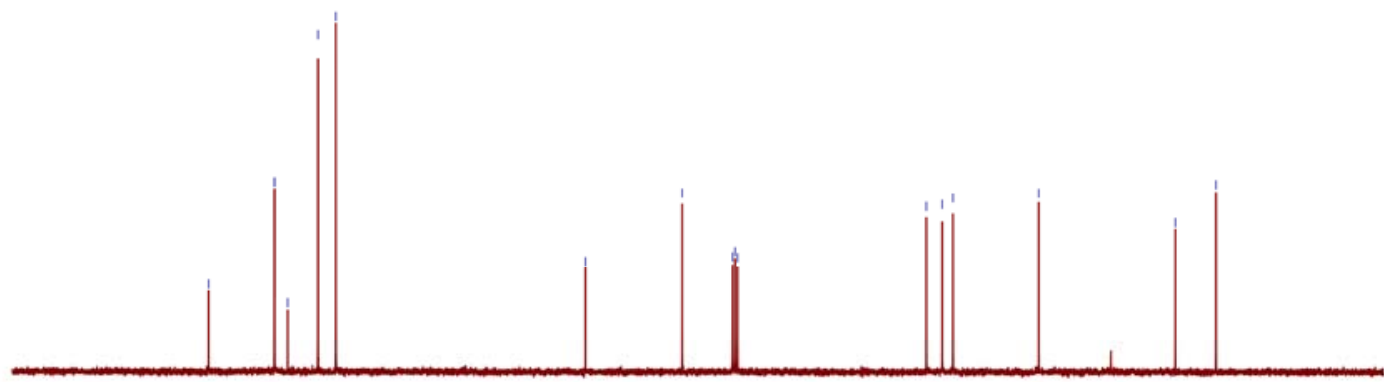

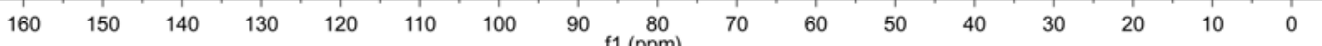




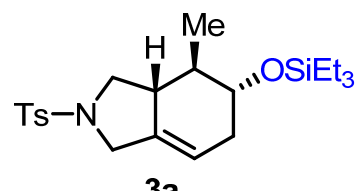

$3 a$

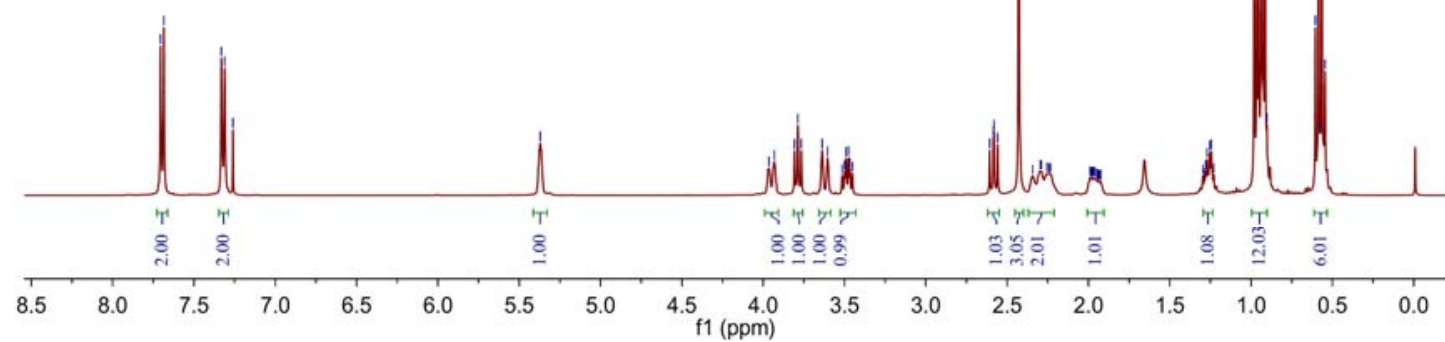

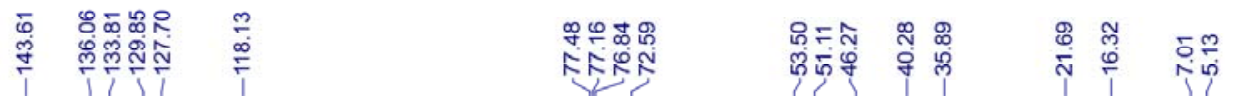<smiles>[3H]N1CC2=CC[C@H](OCC)[C@H](C)[C@@H]2C1</smiles>

$3 a$

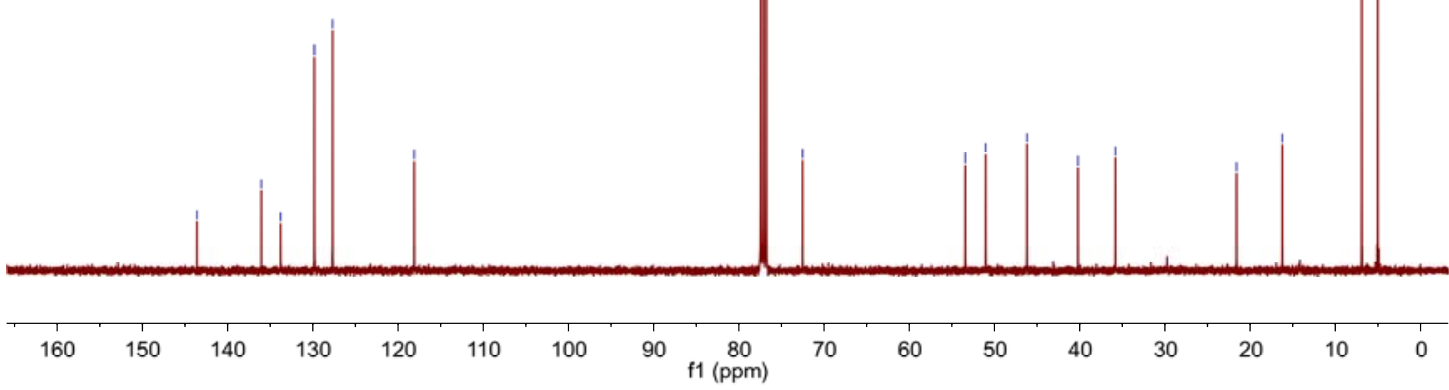




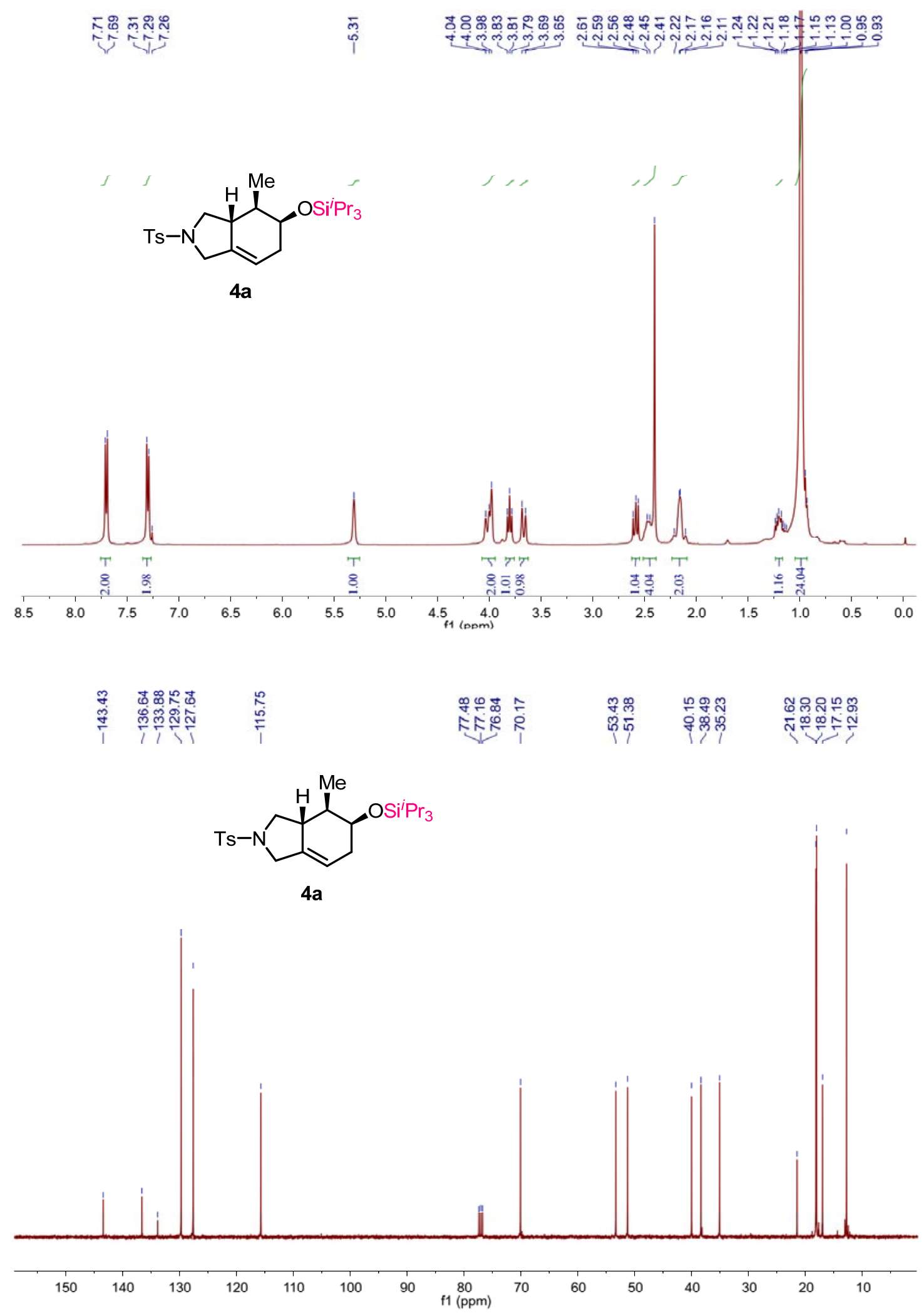


<smiles>[3H]N1CC2=CC[C@H](OCC)C(P)[C@@H]2C1</smiles>

3b

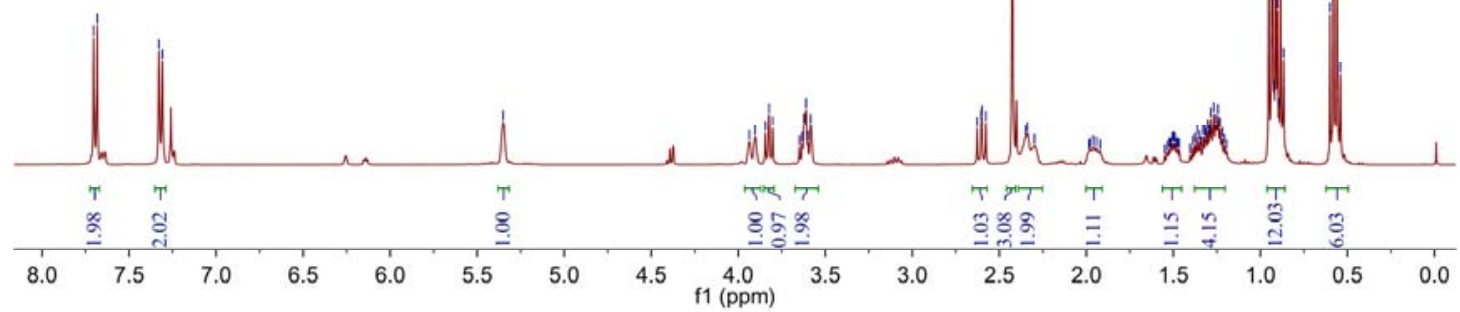

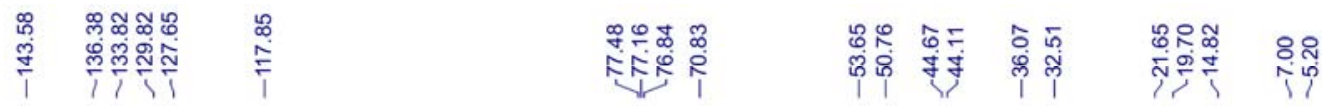<smiles>[3H]N1CC2=CC[C@H](OCC)C(C(C)C)[C@@H]2C1</smiles>

$3 b$

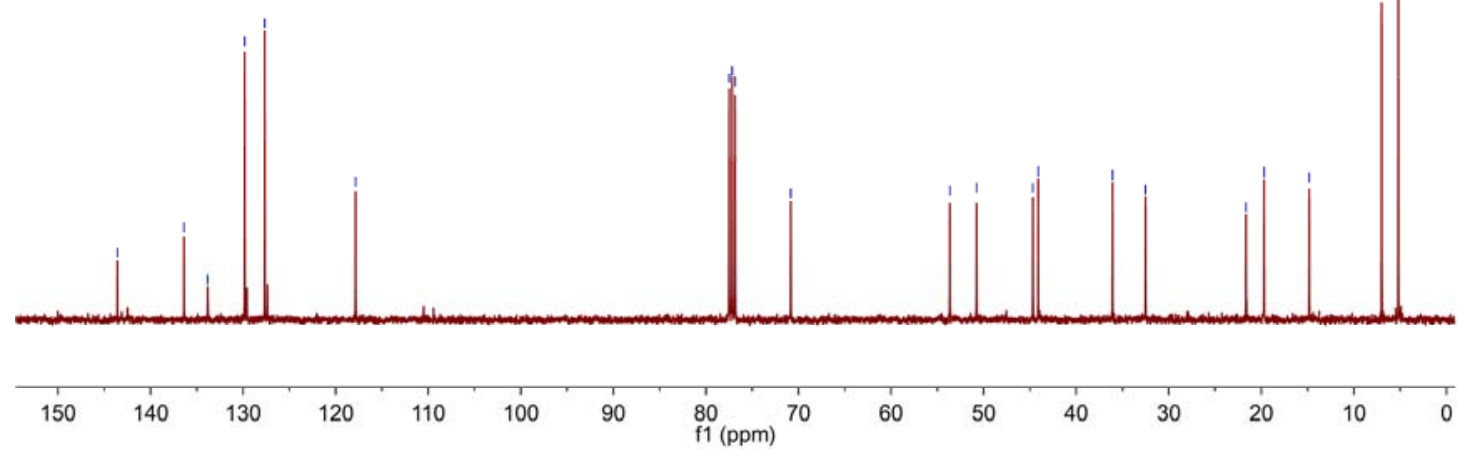




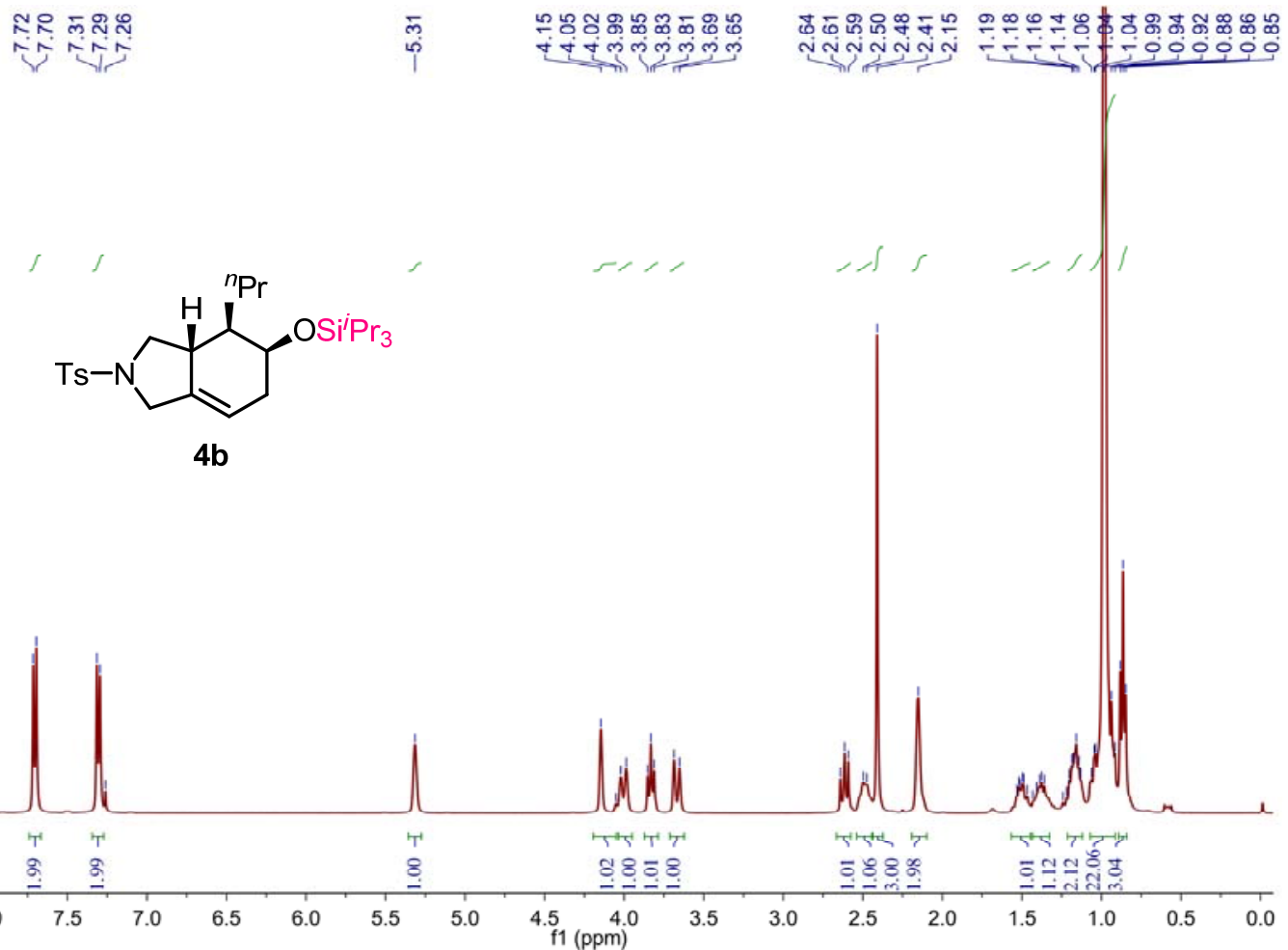

\begin{tabular}{|c|c|c|c|c|c|c|}
\hline 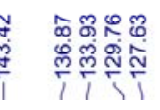 & $\begin{array}{l}\infty \\
\infty \\
\stackrel{6}{1} \\
\end{array}$ & 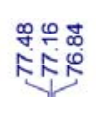 & $\begin{array}{l}0 \\
0 \\
0 \\
1\end{array}$ & हृల్ల & 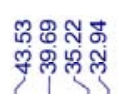 & 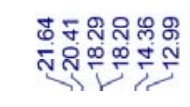 \\
\hline
\end{tabular}

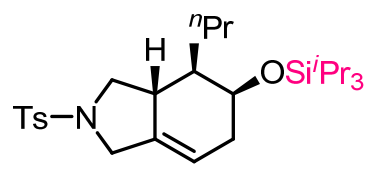

4b

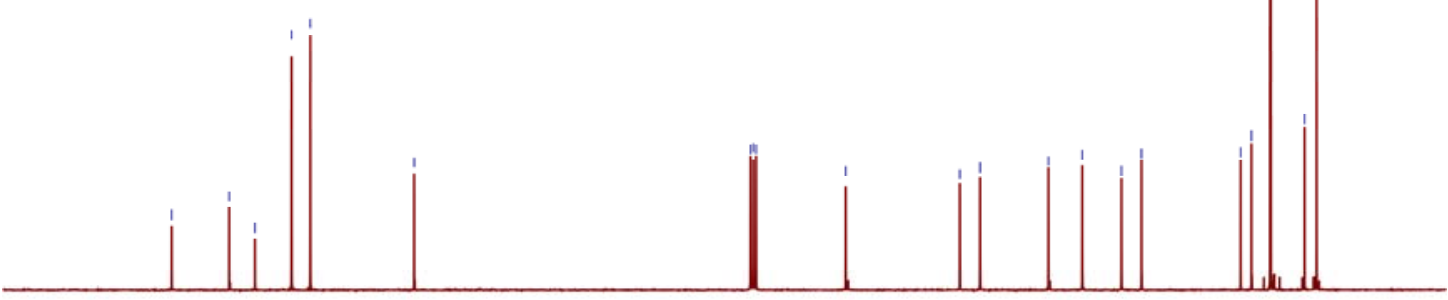

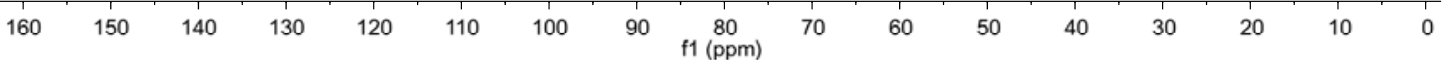



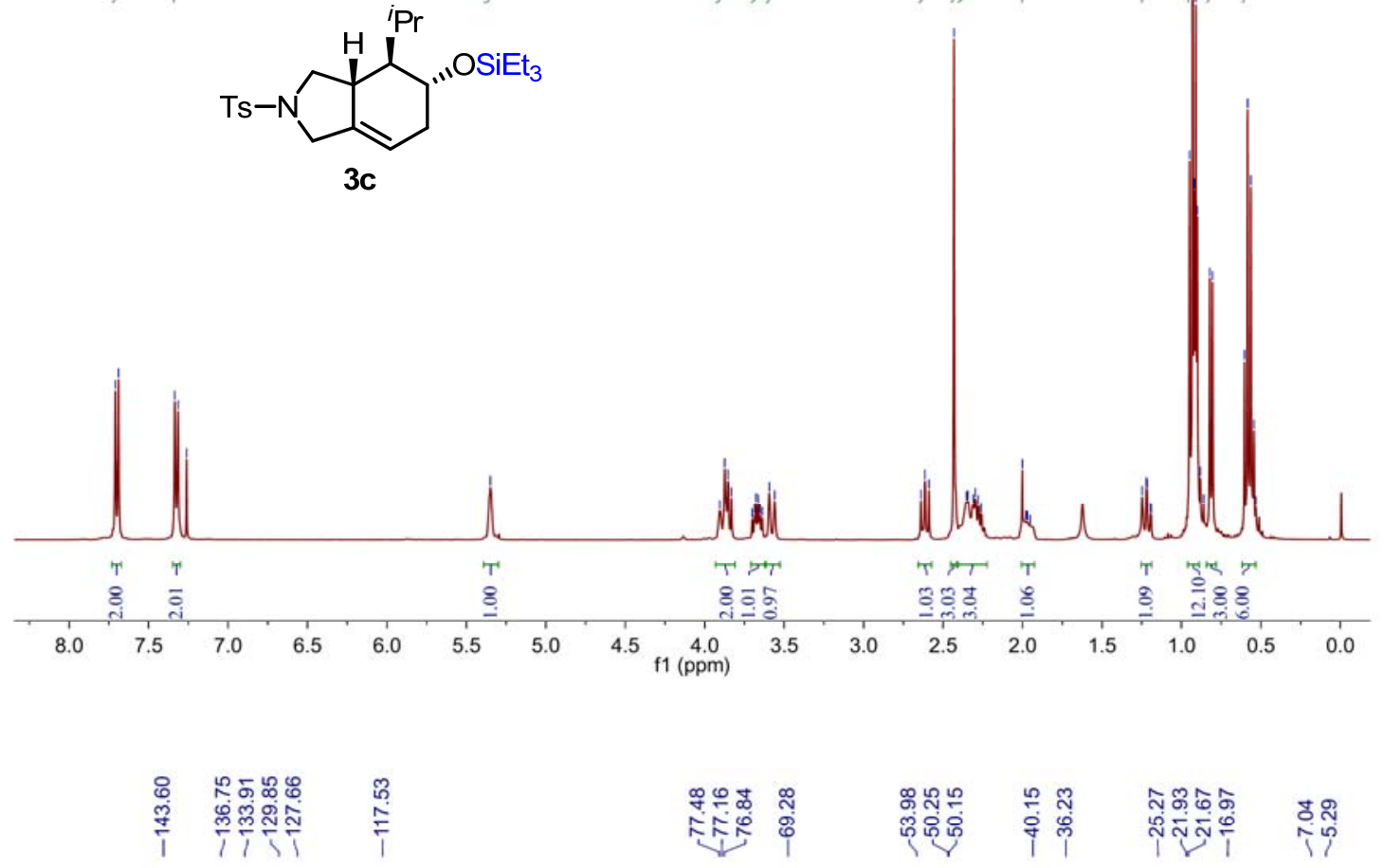<smiles>CCO[C@H]1CC=C2CN([As])C[C@H]2[C@H]1C(C)C</smiles>

3c

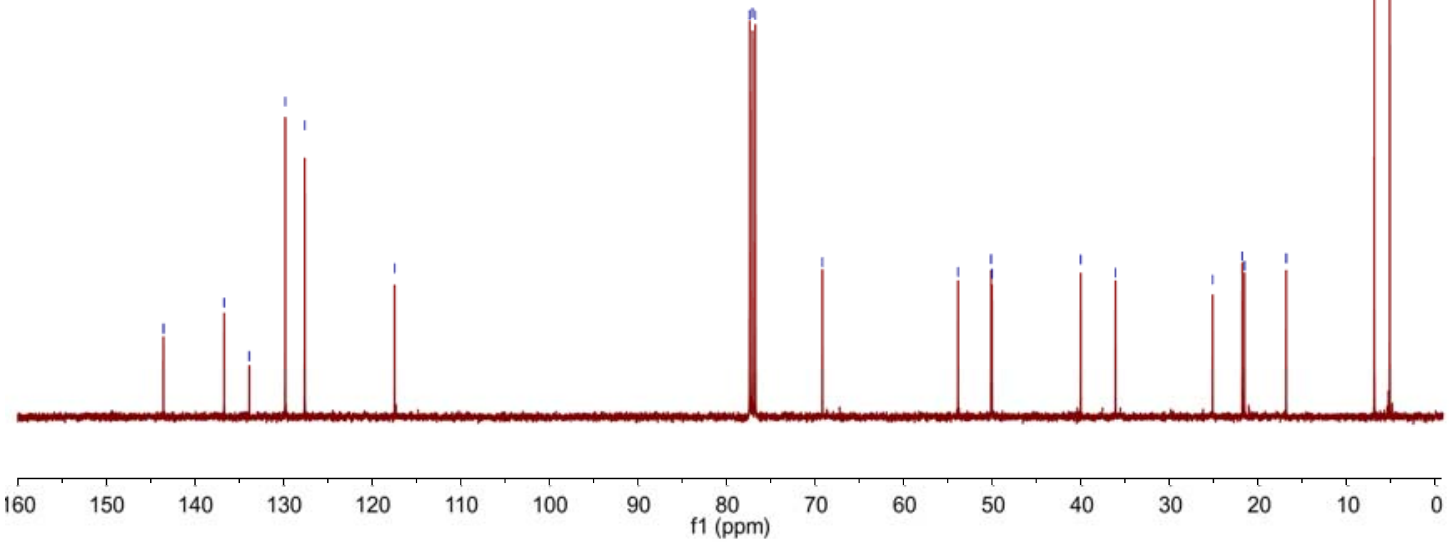



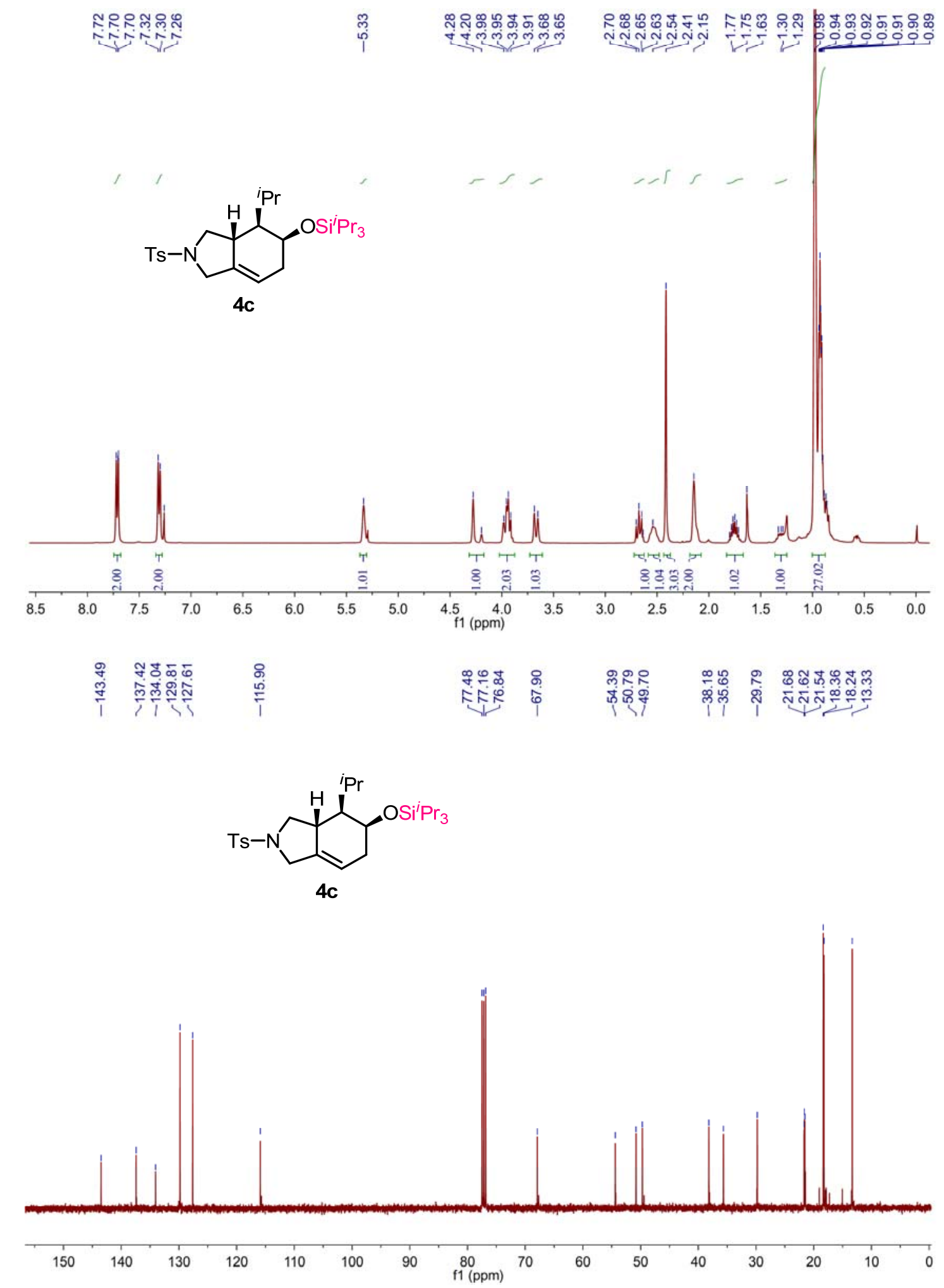

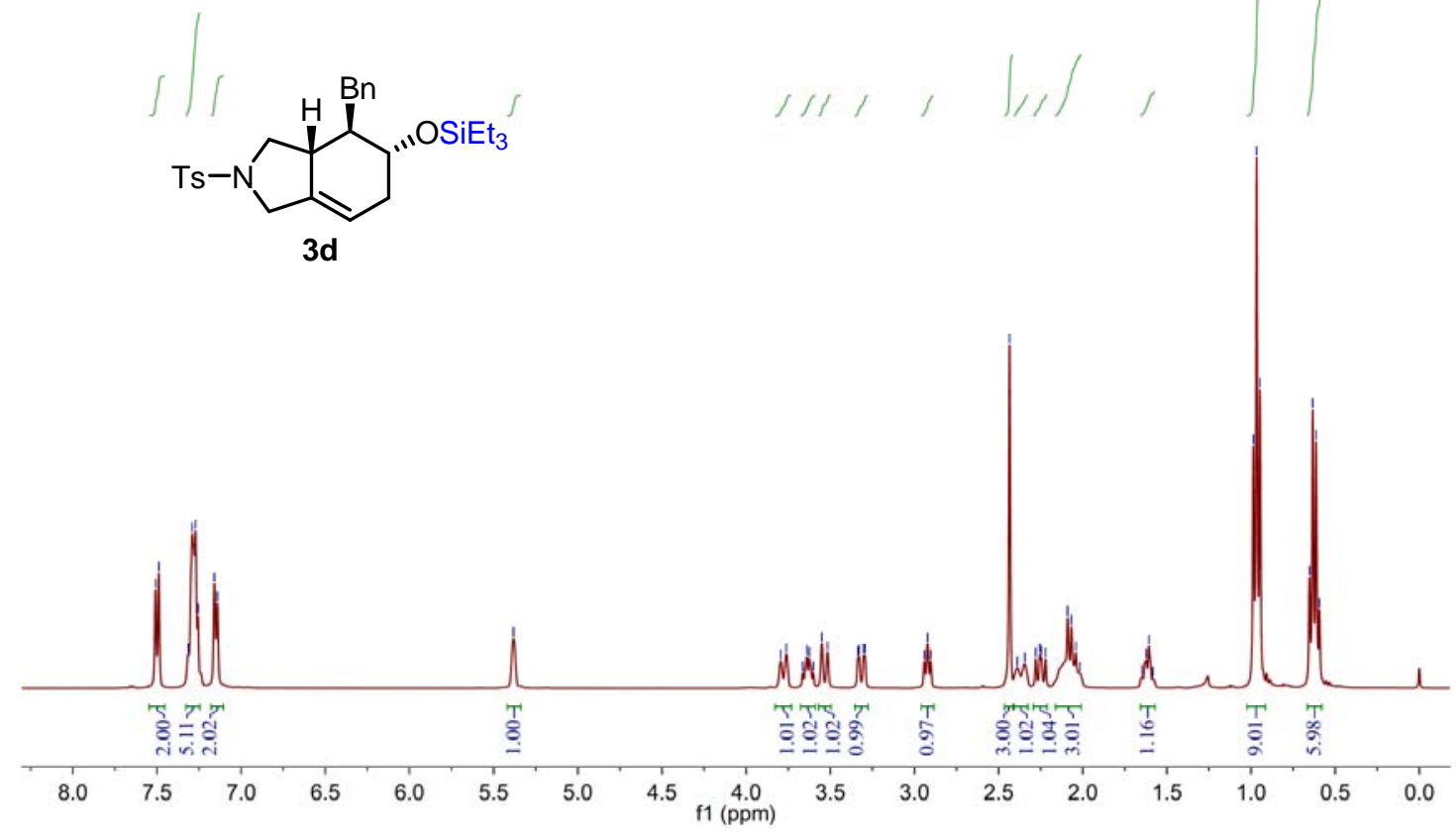

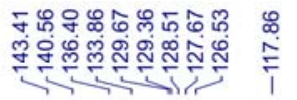

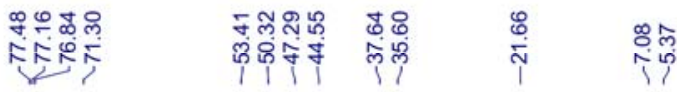<smiles>[3H]N1CC2=CC[C@H](OCC)[C@H](Br)C2C1</smiles>

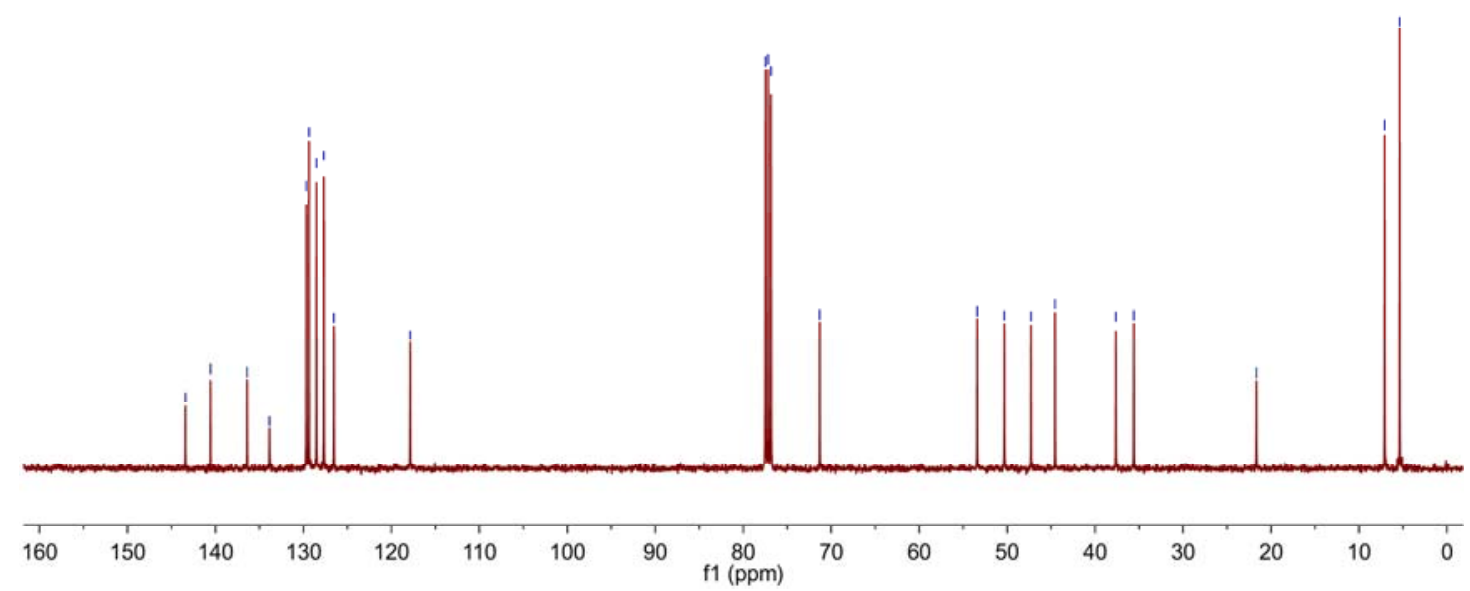




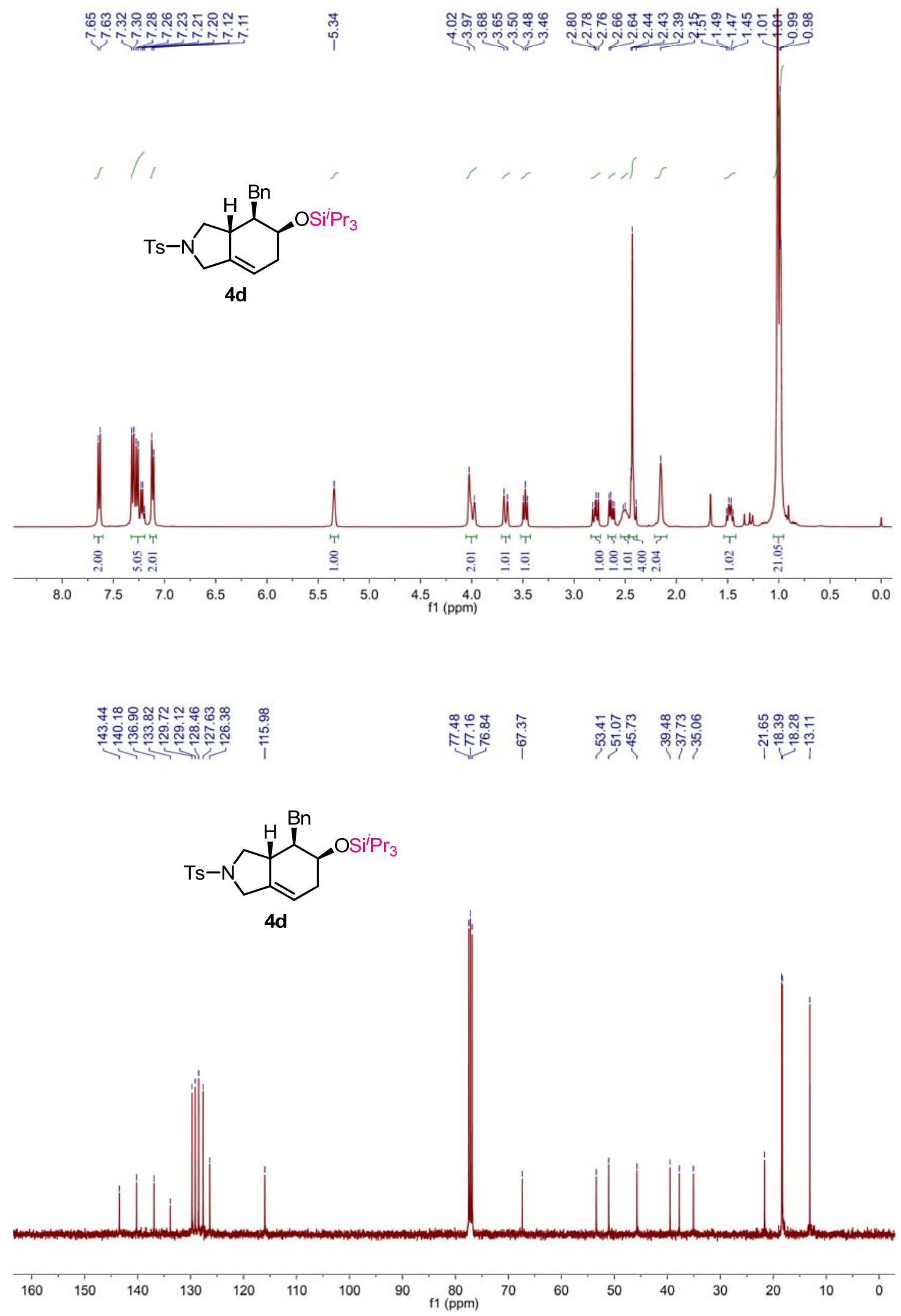




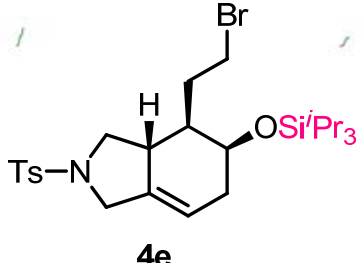

$4 e$

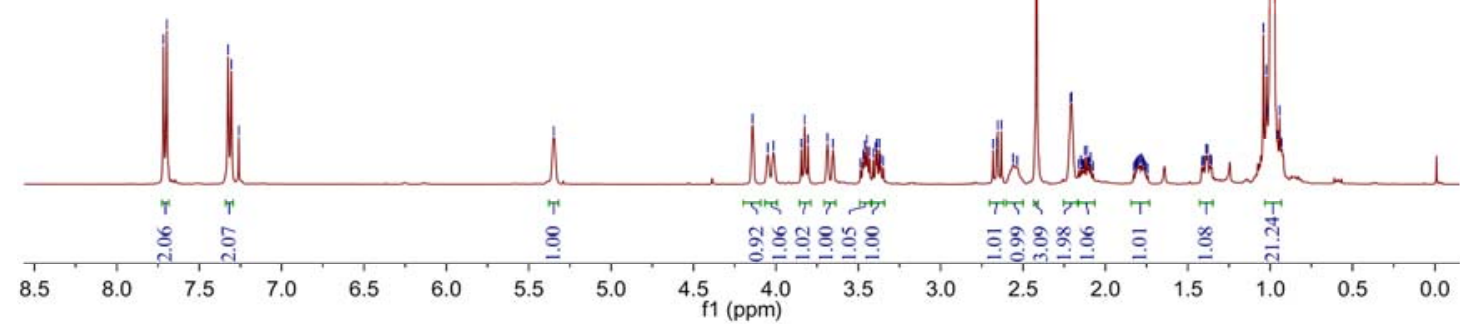

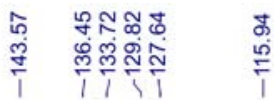

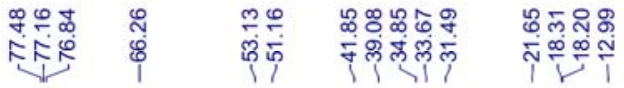

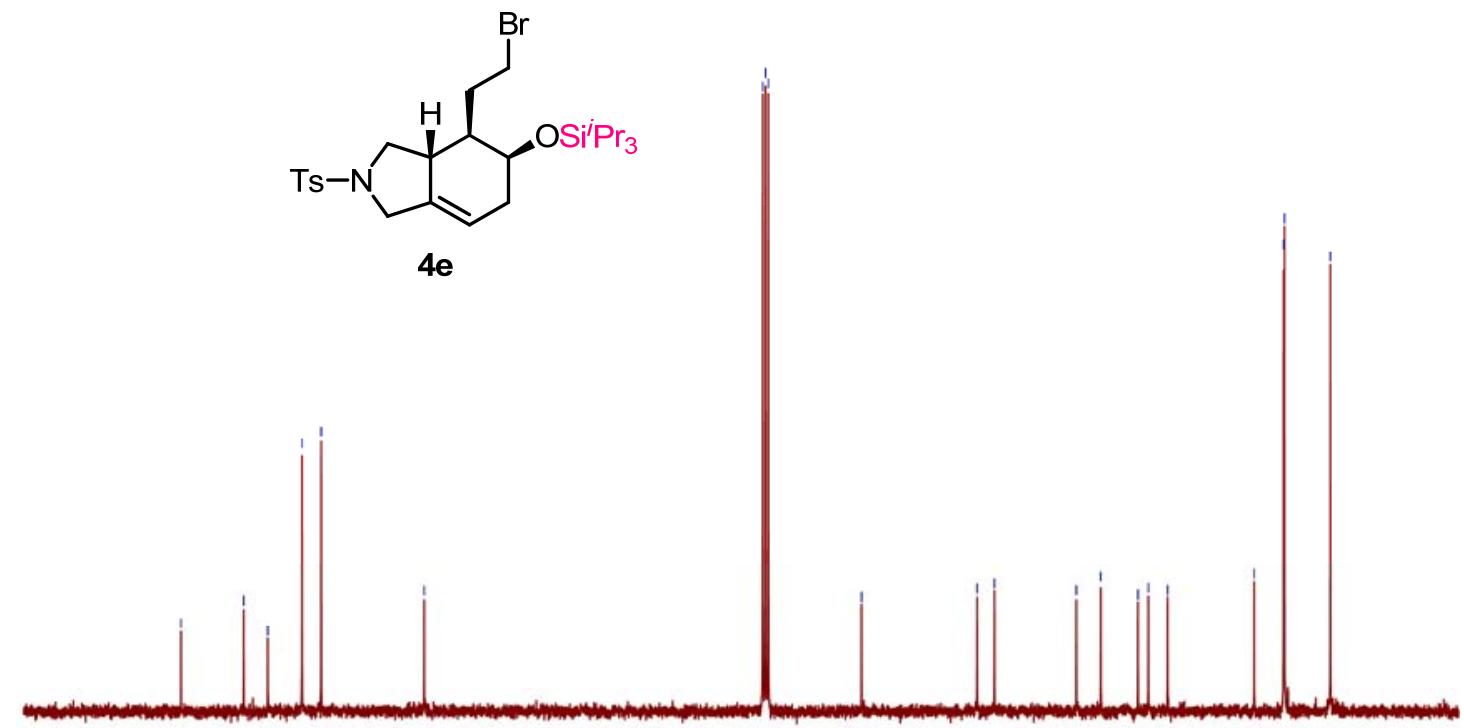

$4 e$
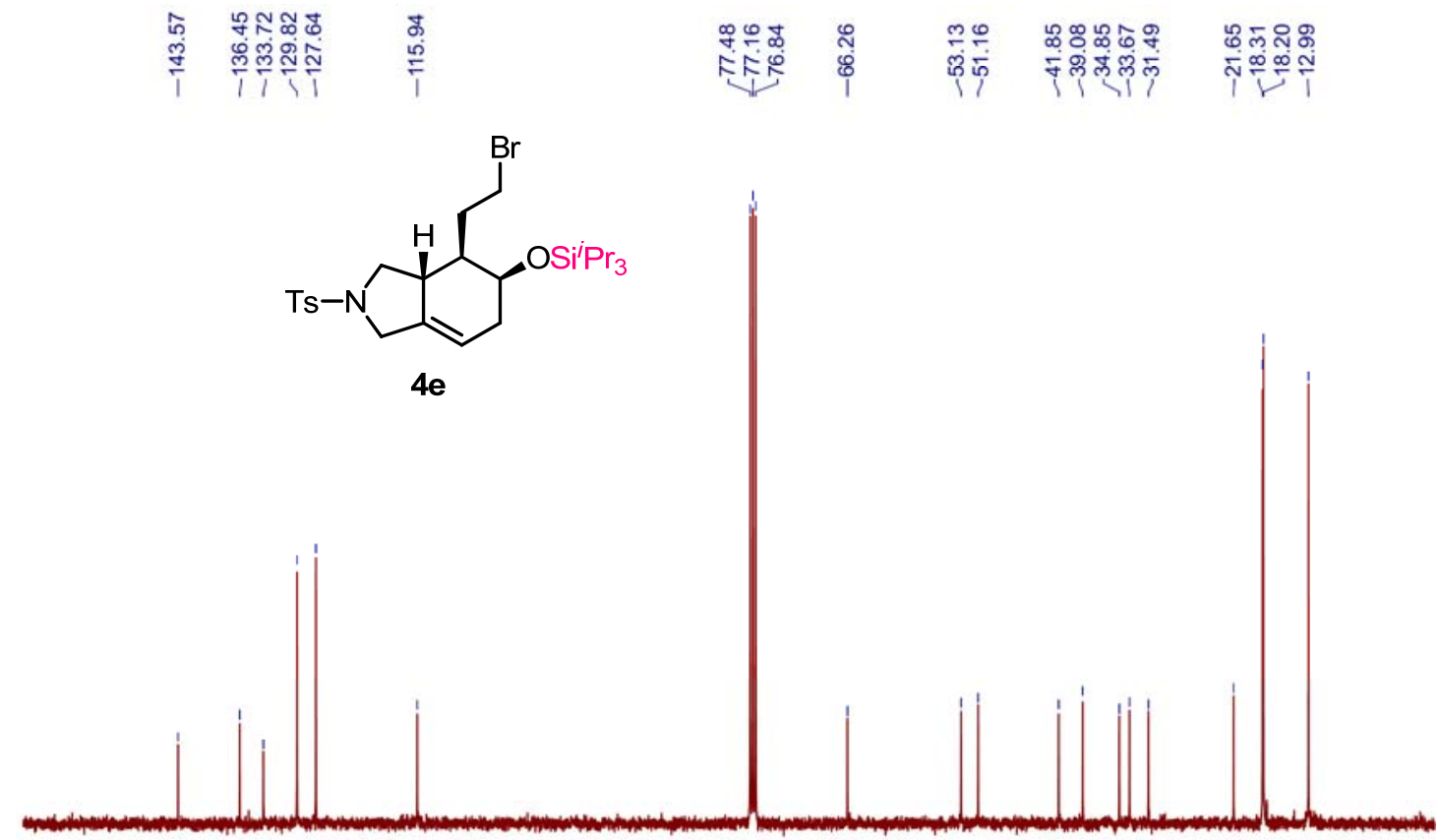


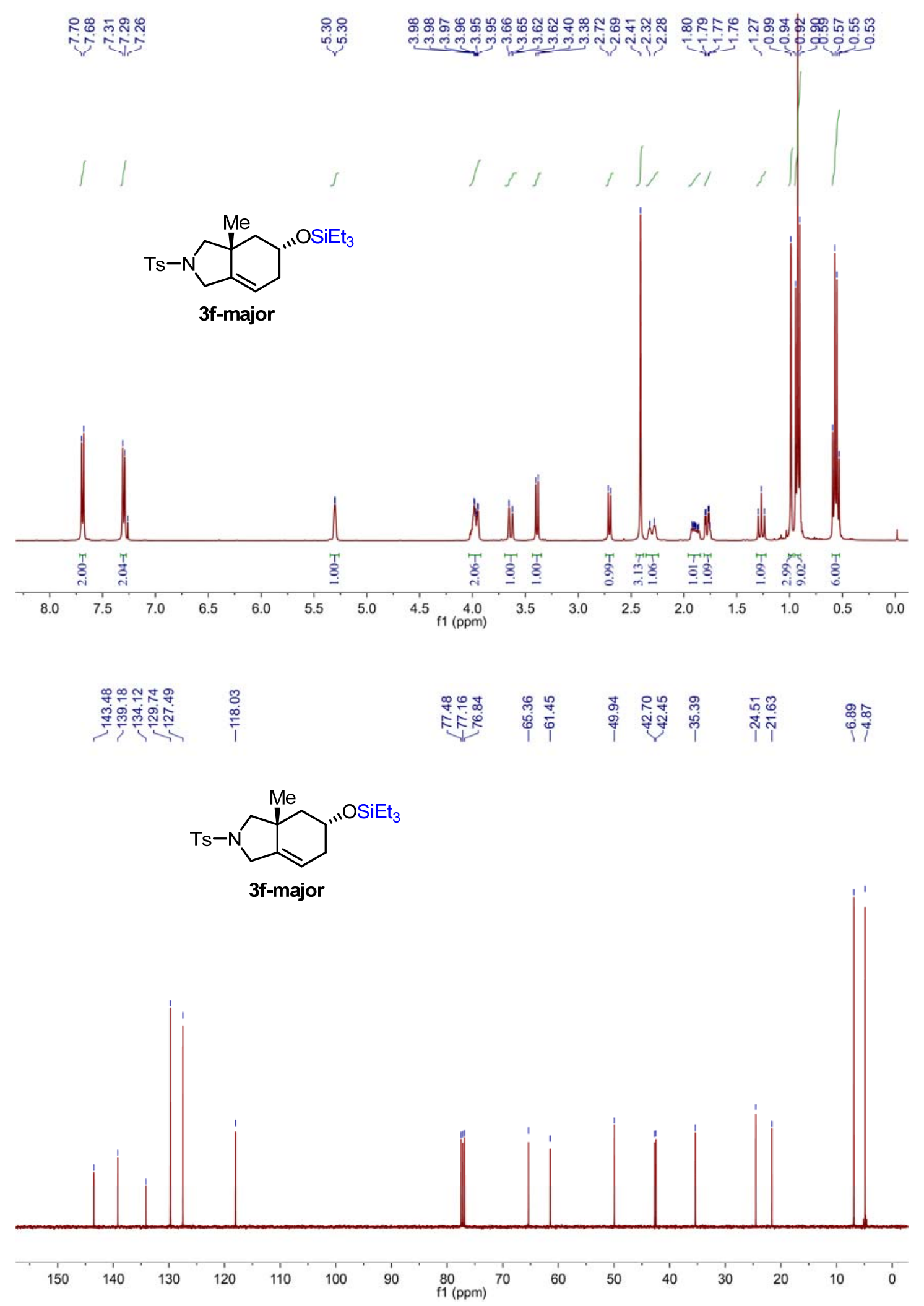




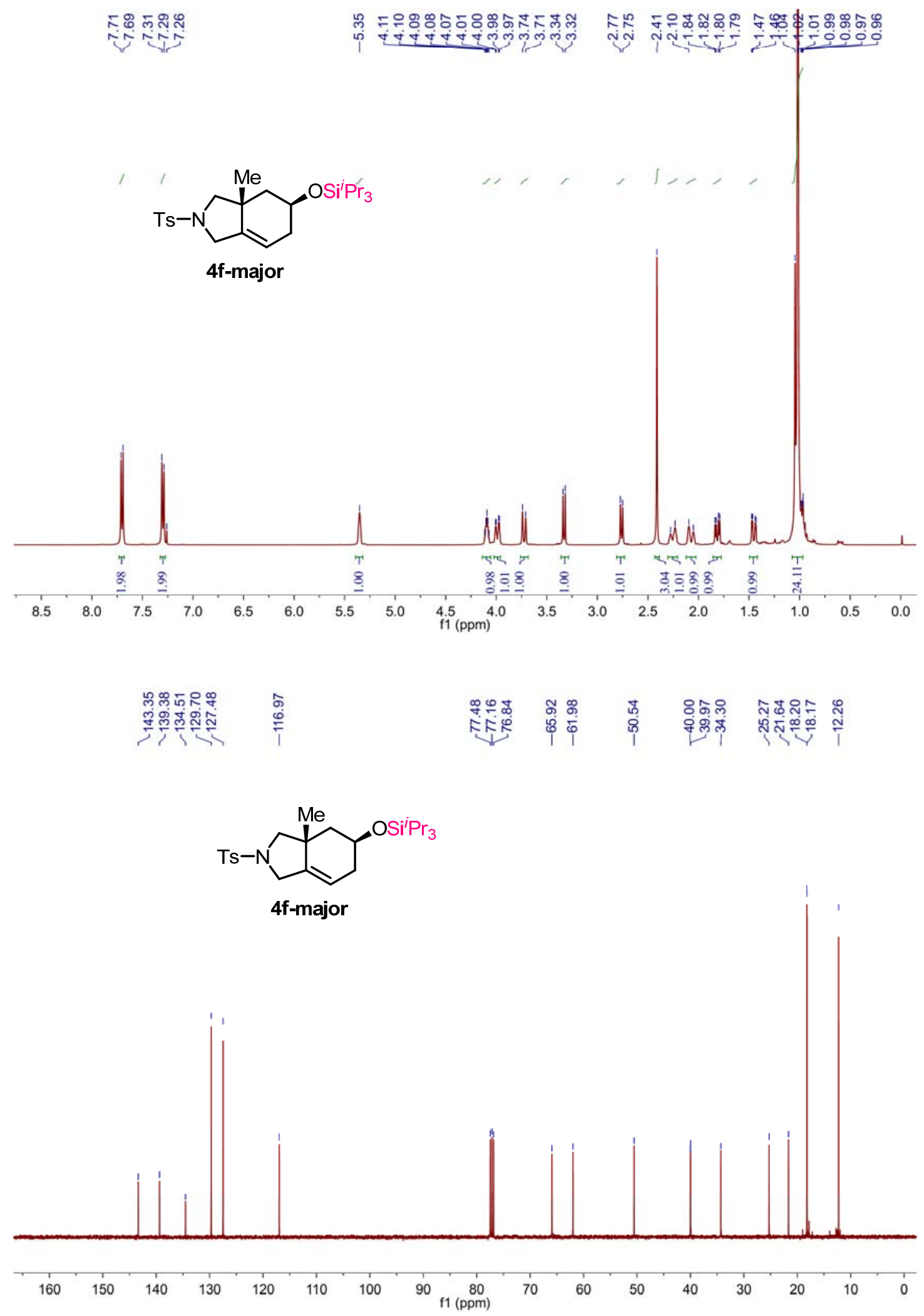




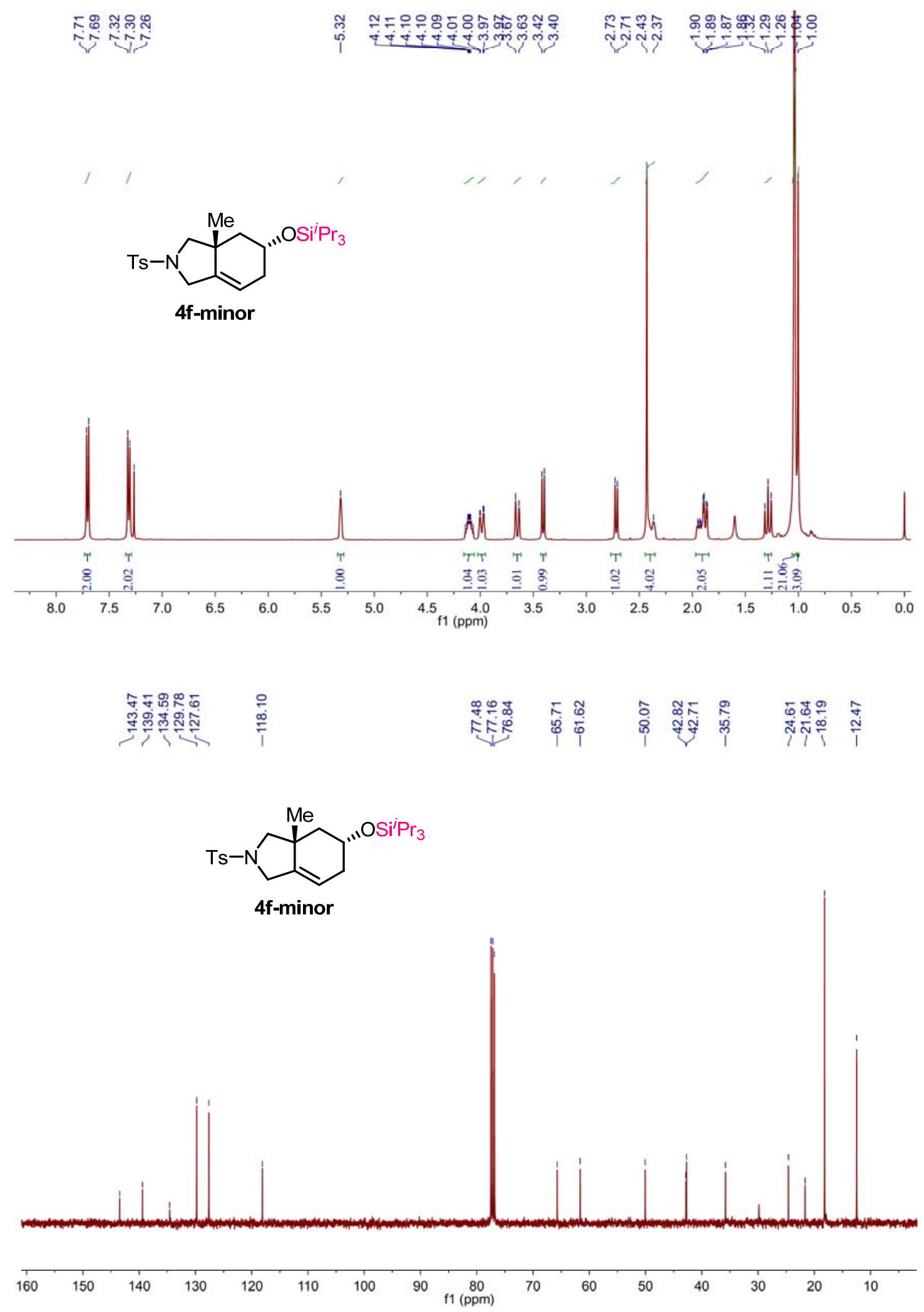




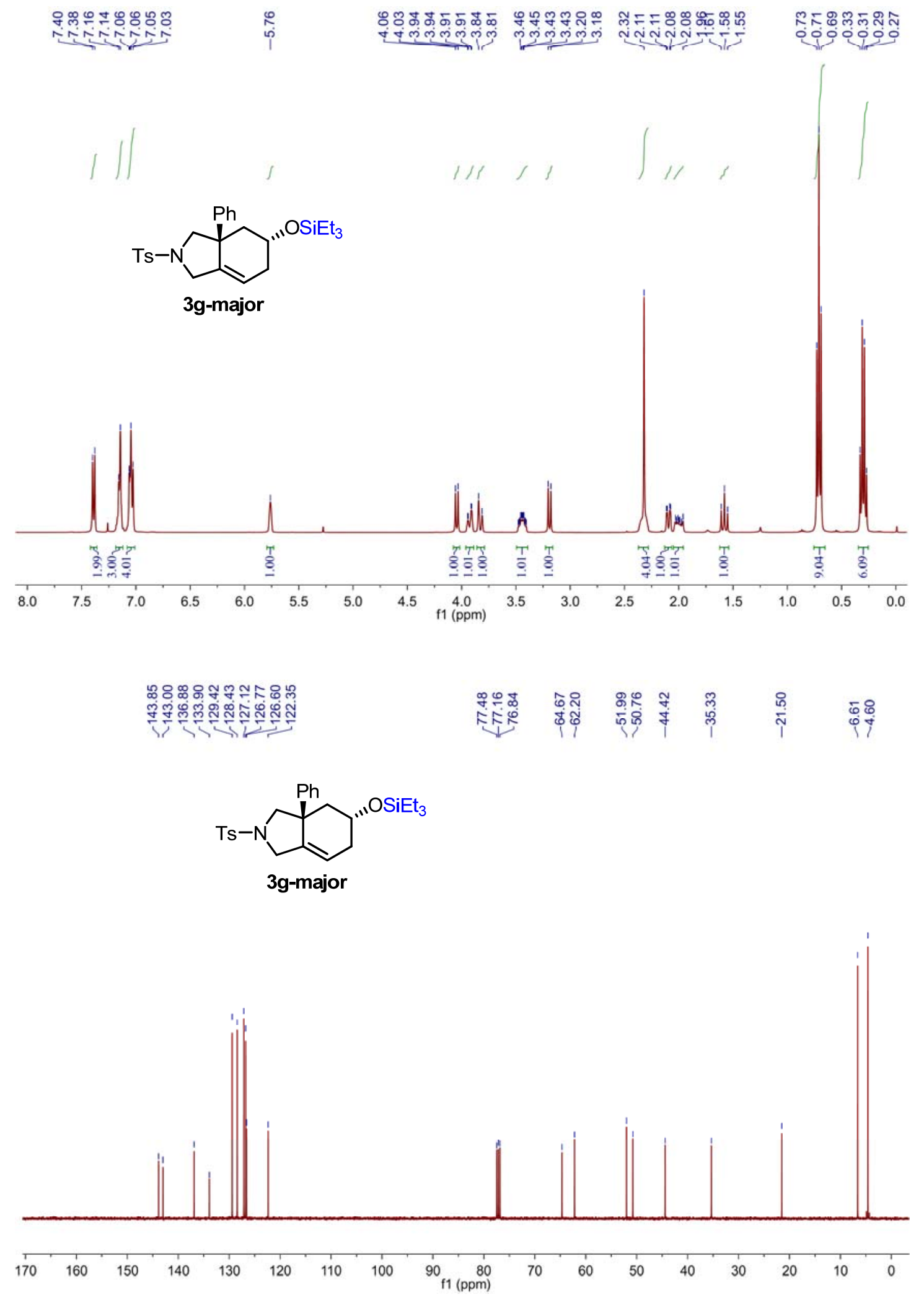




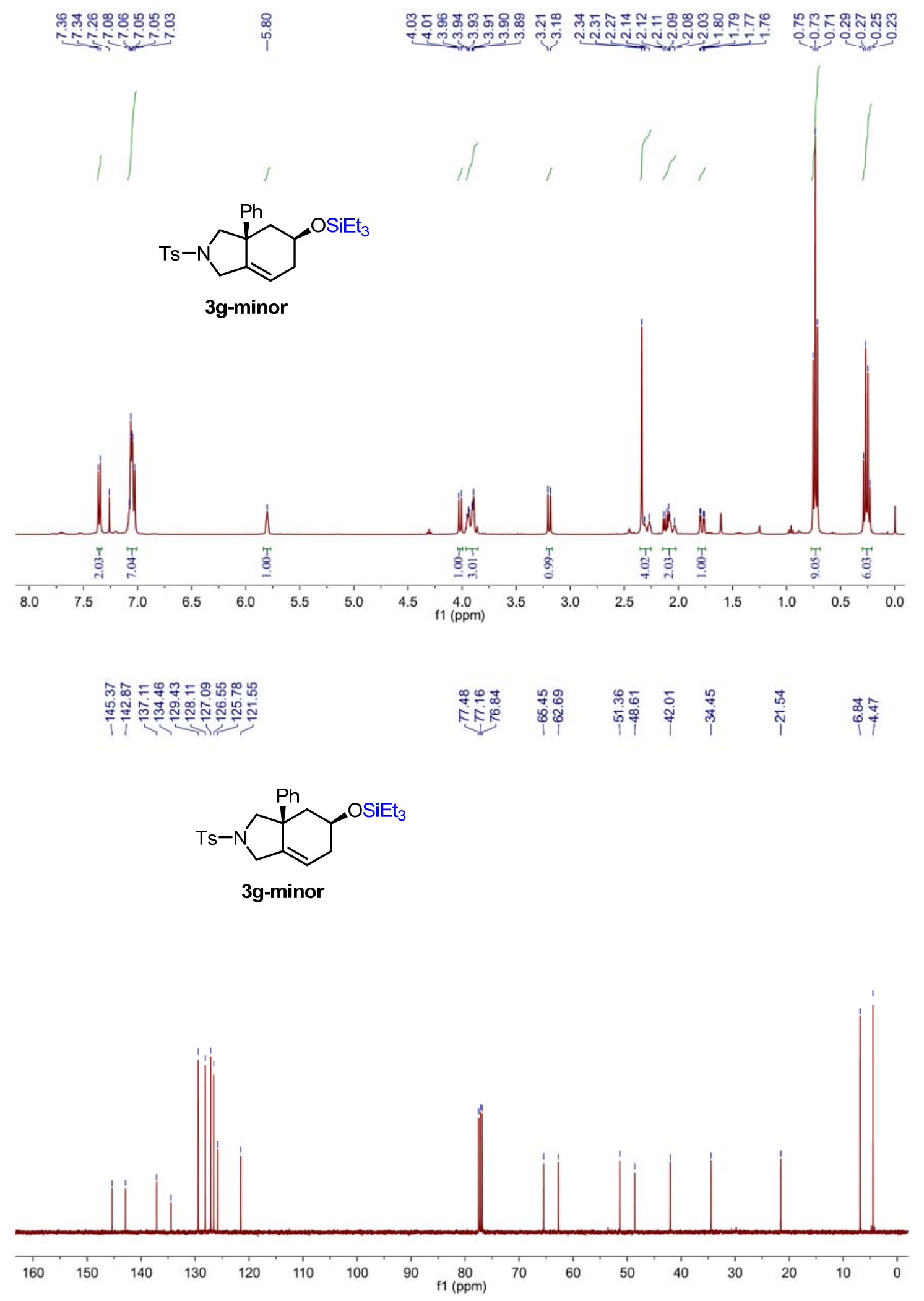




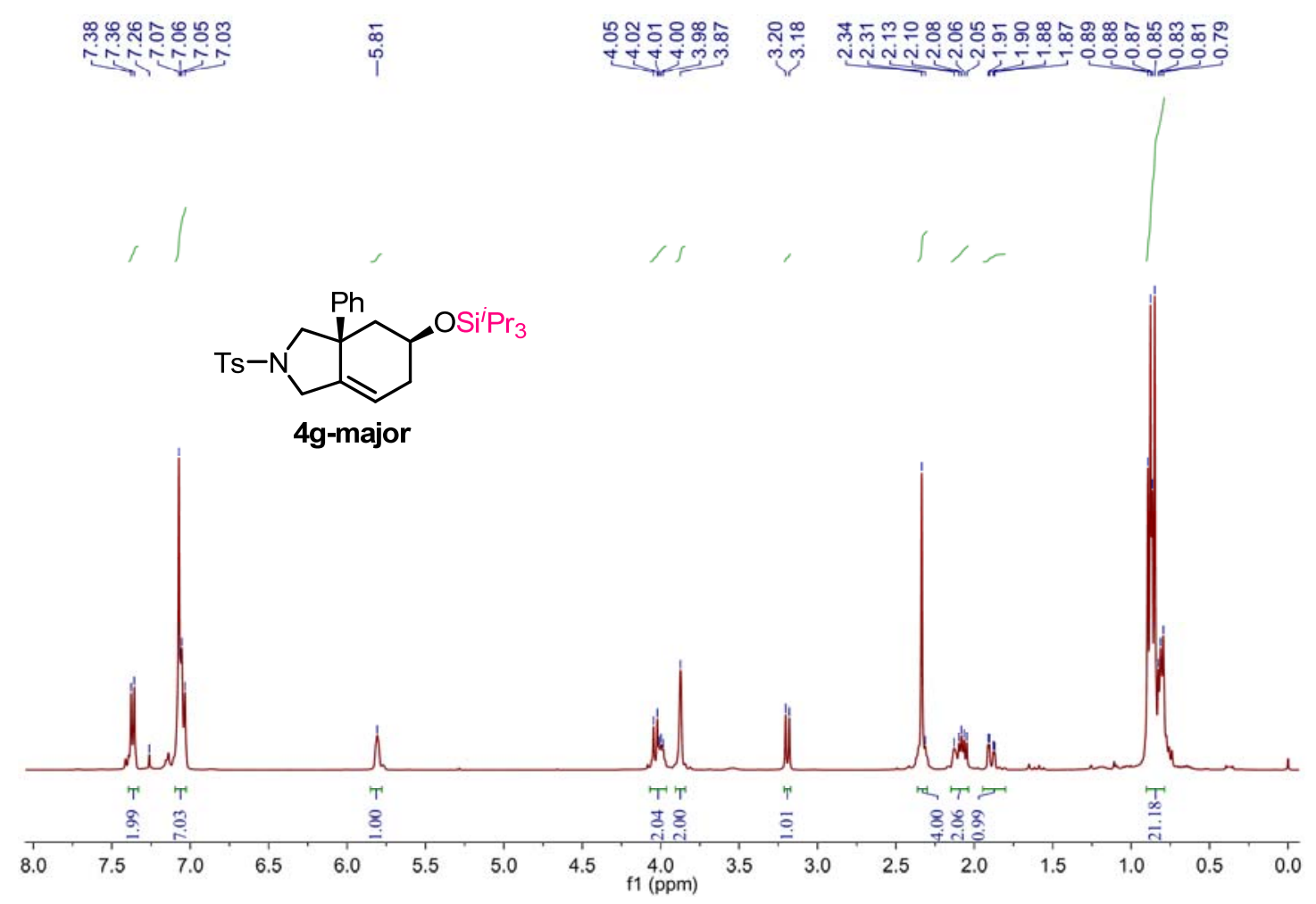

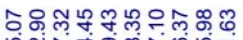

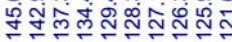

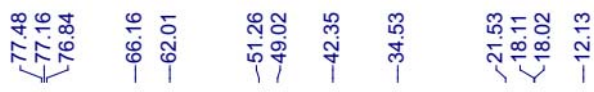
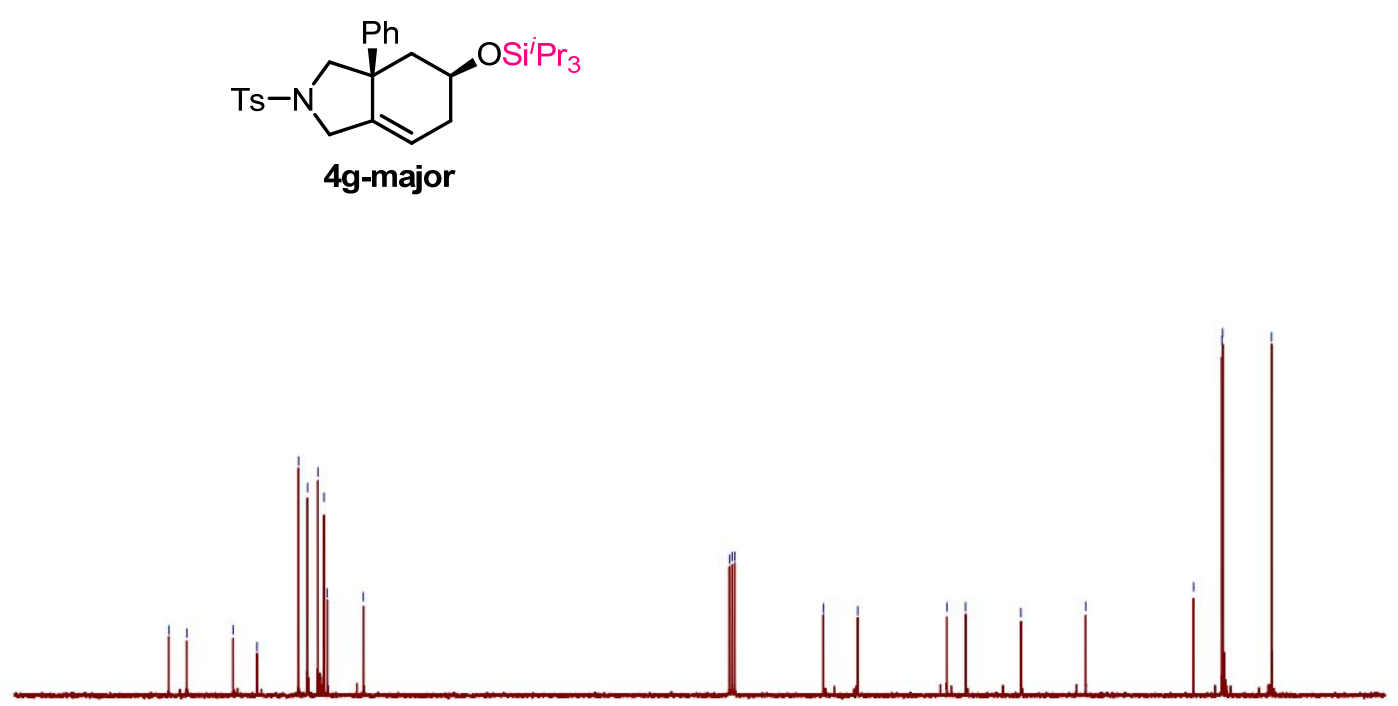

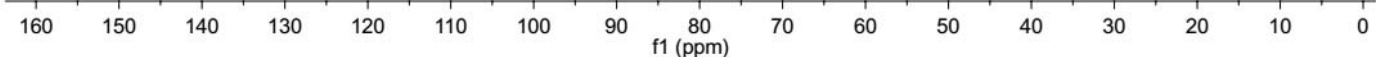



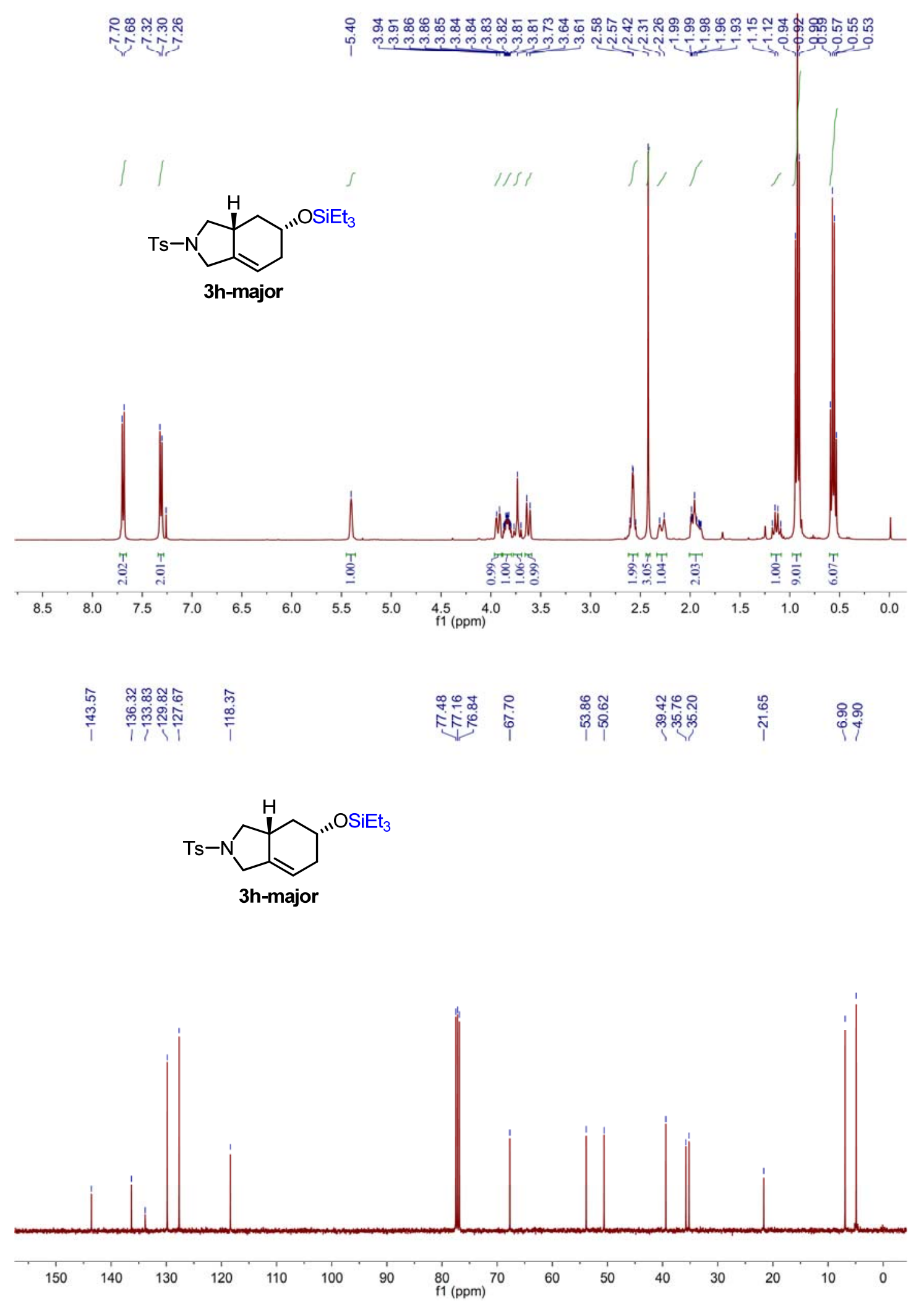


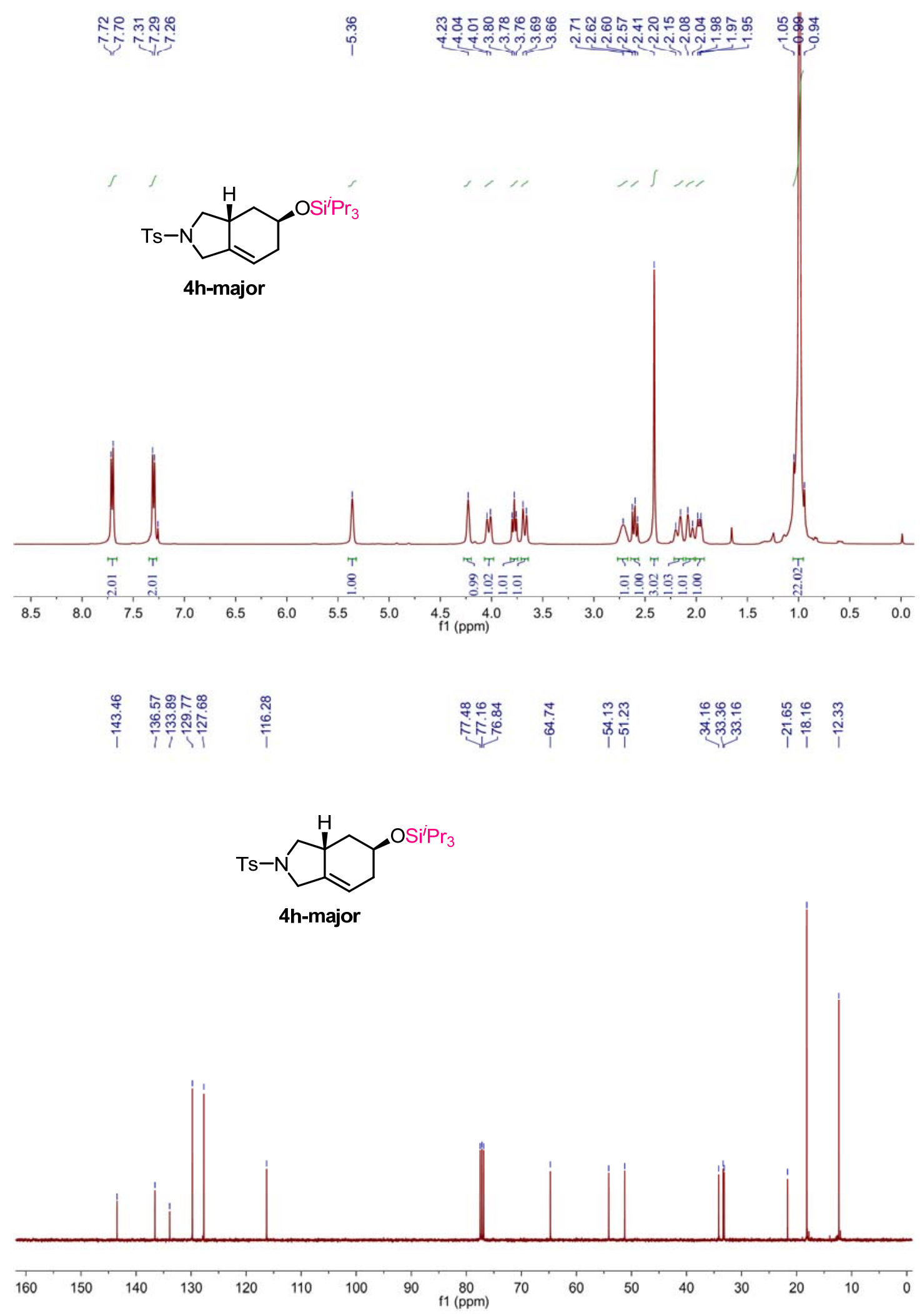




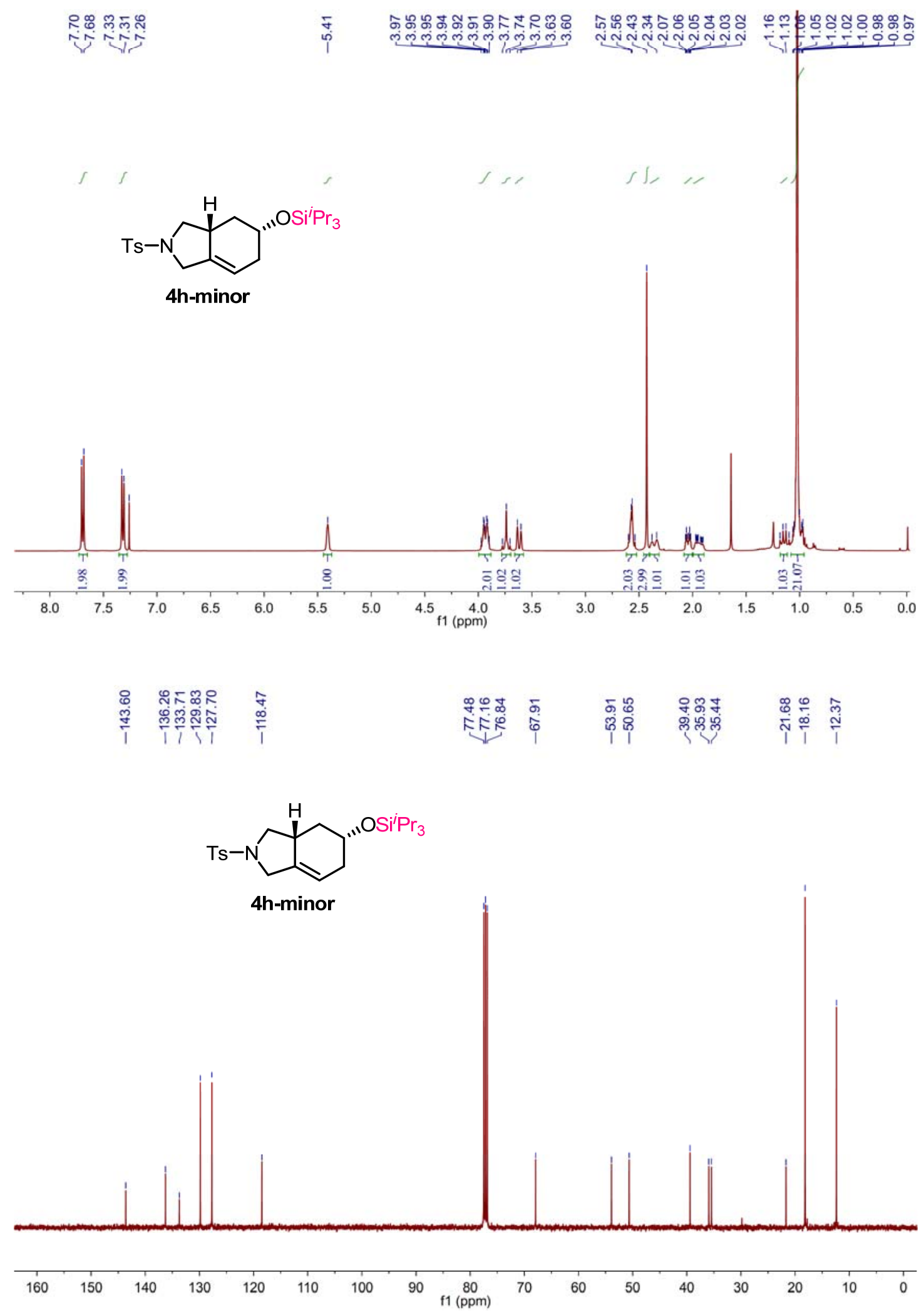




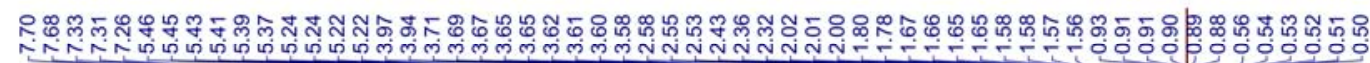

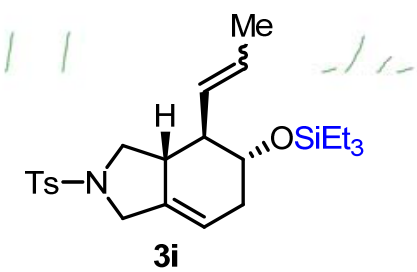

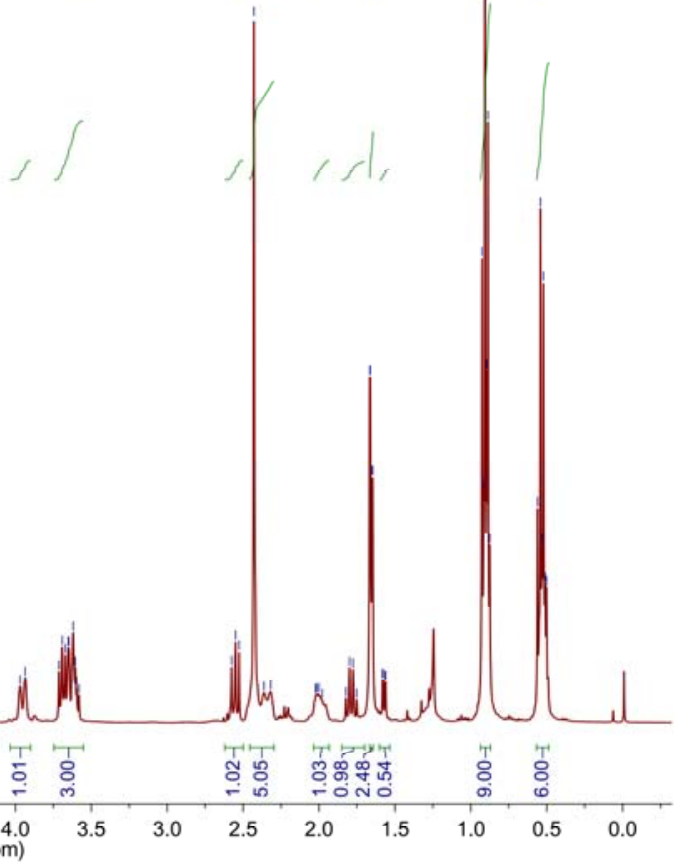

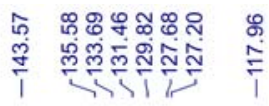

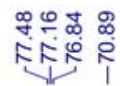

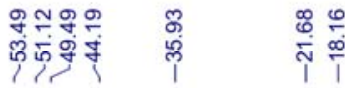

$\frac{0}{\frac{\infty}{\infty}} \quad$ \&5<smiles>[Y5]N1CC2=CC[C@H](OCC)[C@H](C=C[Y4]([H])=O)[C@H]2C1</smiles>

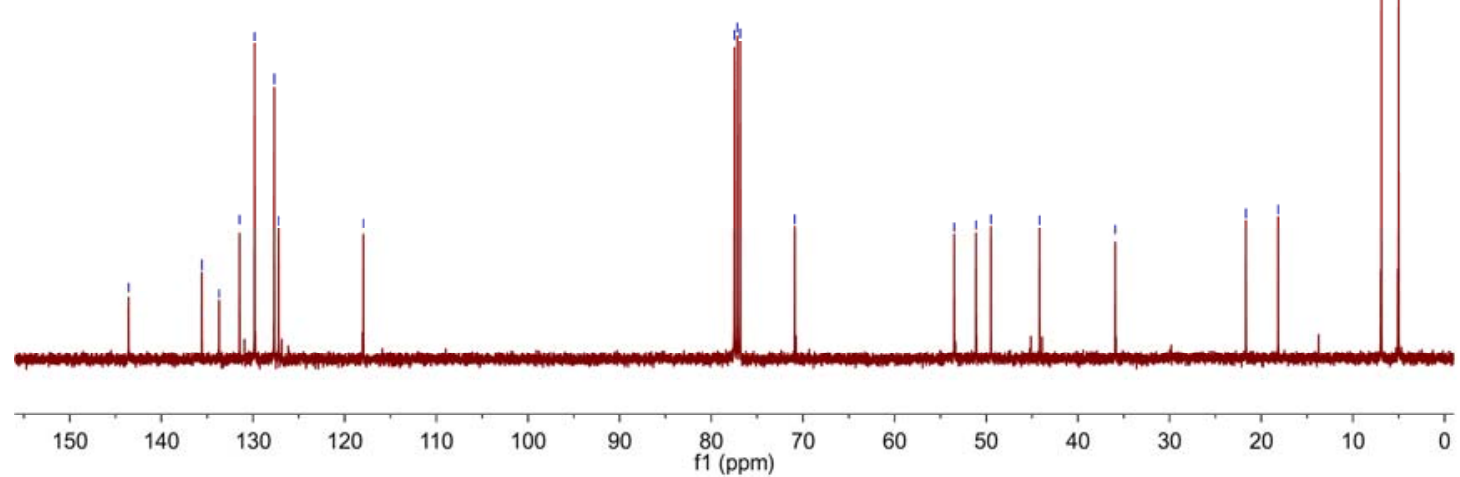




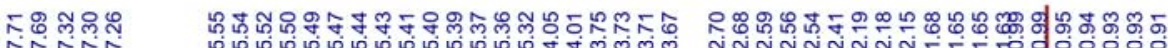

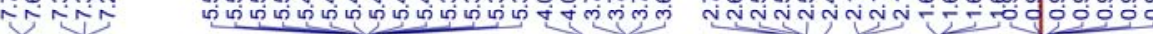<smiles>CC=C[C@@H]1[C@@H]2CN([AsH])CC2=CC[C@H]1O[GaH2]</smiles>

4i

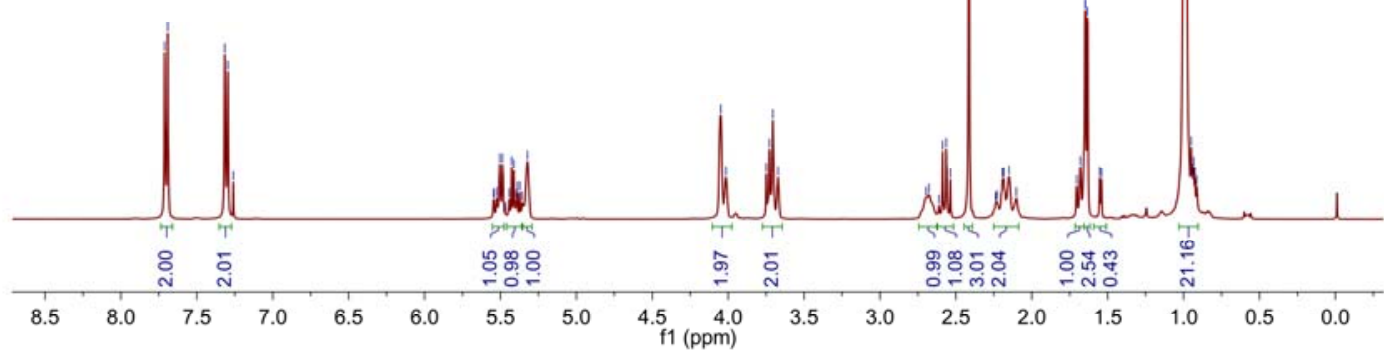

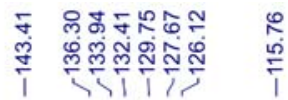

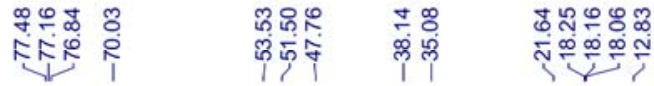<smiles>CC=C[C@@H]1[C@@H]2CN([As])CC2=CC[C@H]1O[Al-]</smiles>

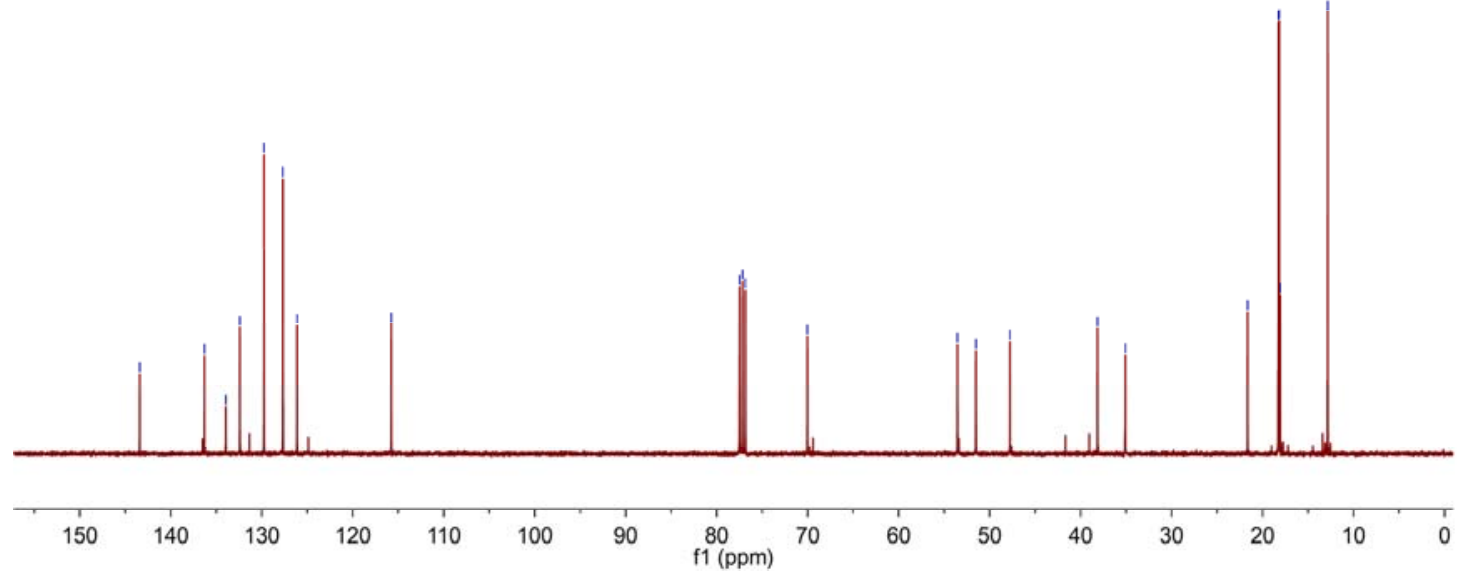




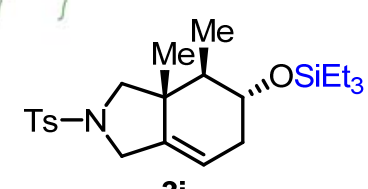

3j
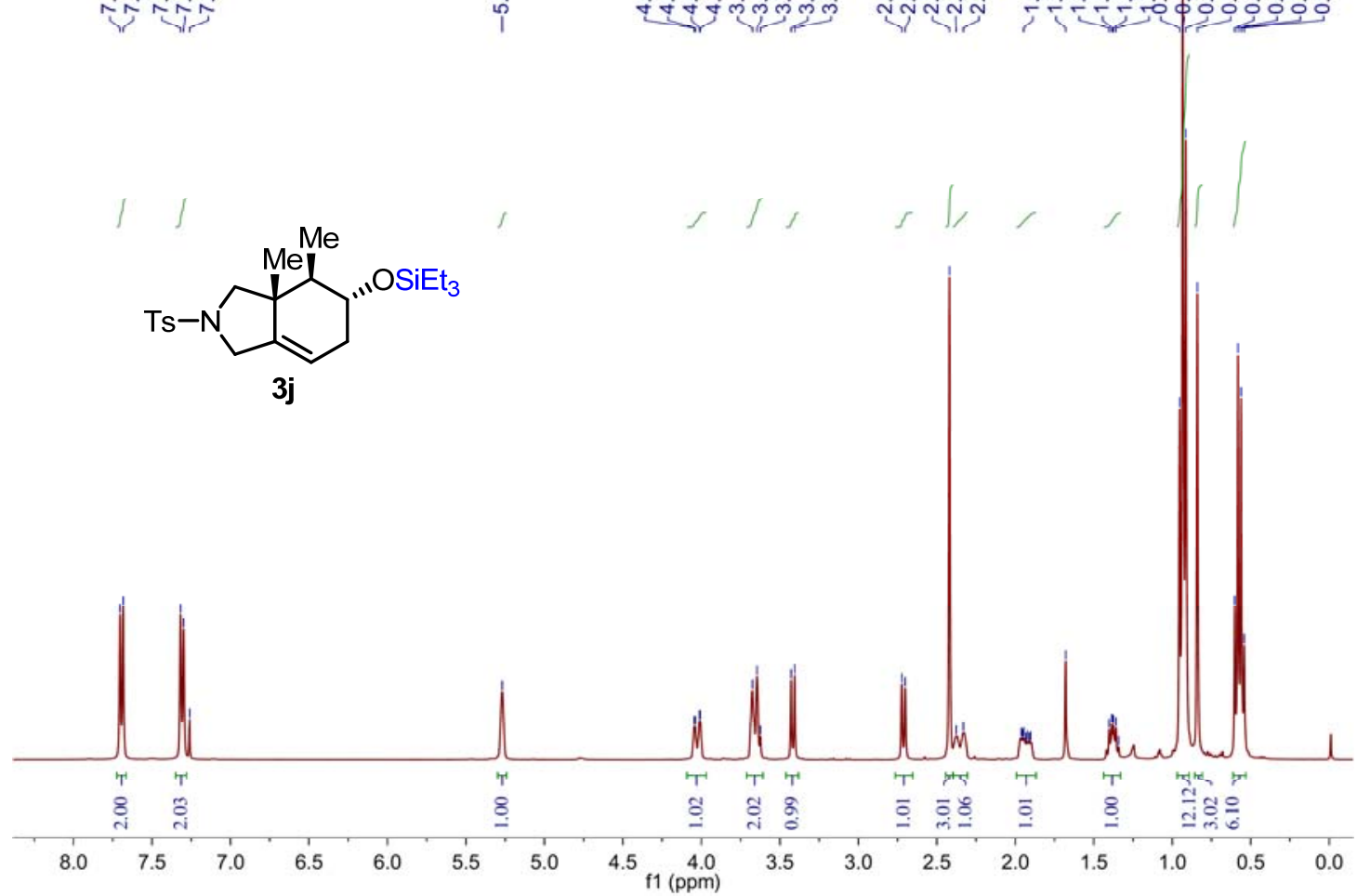

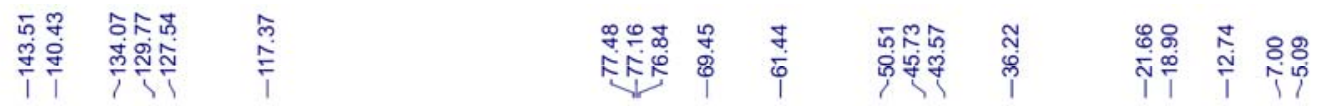<smiles>[3H]N1CC2=CC[C@H](OCC)C(C)C2(C)C1</smiles>

3j

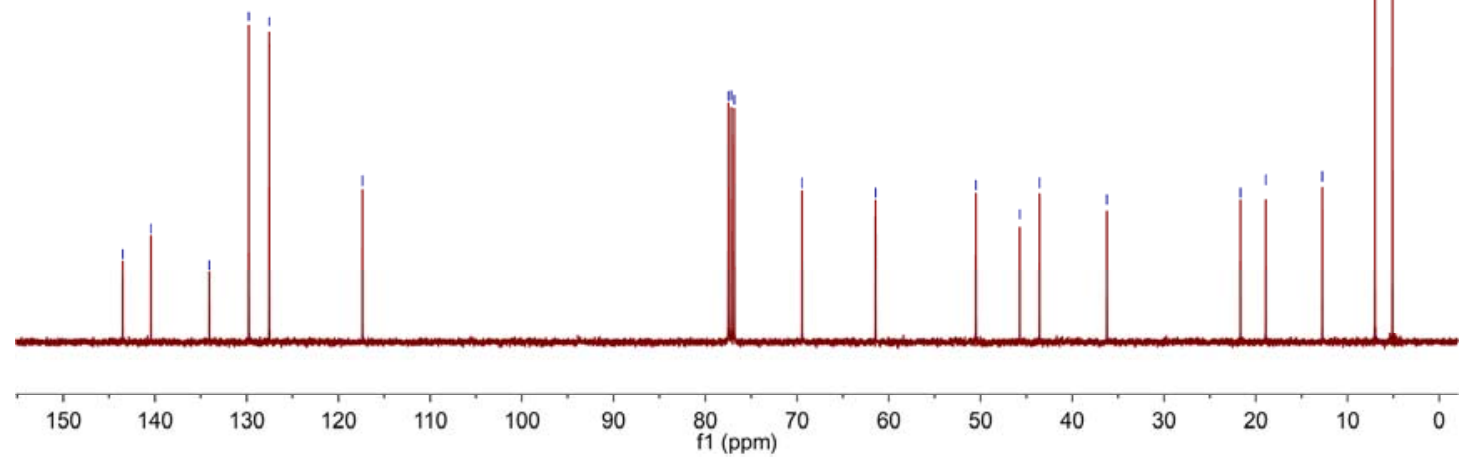




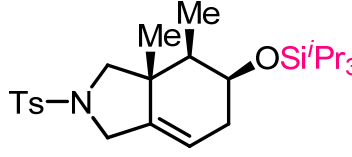

4j

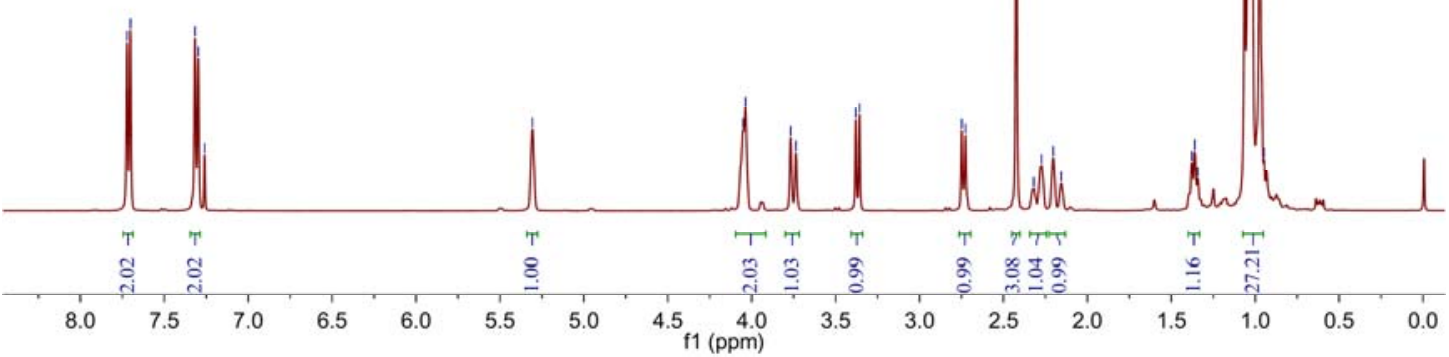

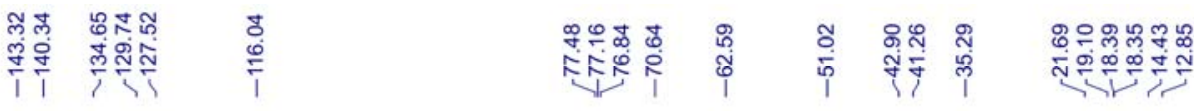<smiles>[M]C12CN([AsH])CC1=CCC(O[SiH2])C2C</smiles>

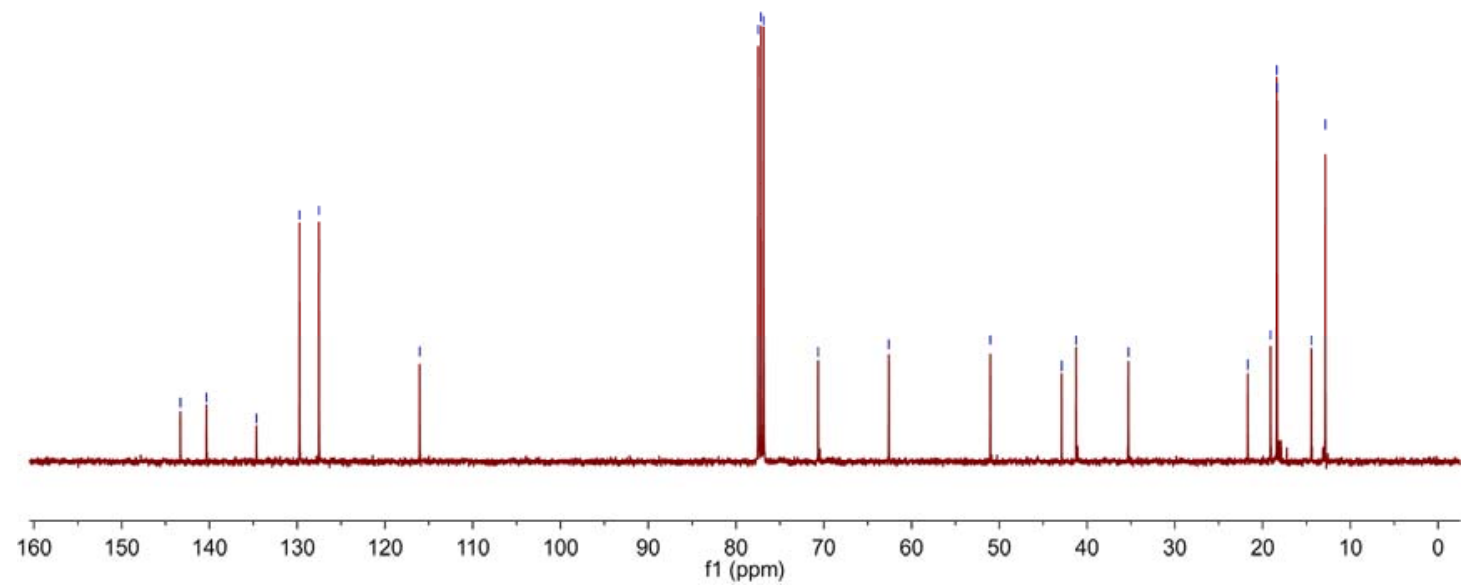


<smiles>[3H]N1CC2=CC[C@H](OCC)C(c3ccccc3)[C@@H]2C1</smiles>

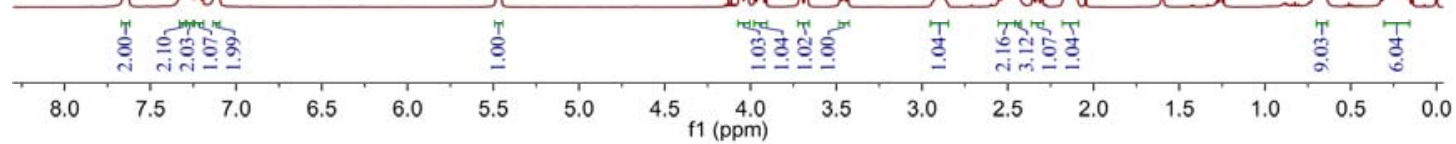

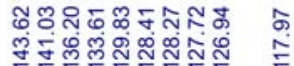<smiles>[3H]N1CC2=CC[C@H](OCC)[C@@H](c3ccccc3)C(c3ccccc3)[C@@H]2C1</smiles>

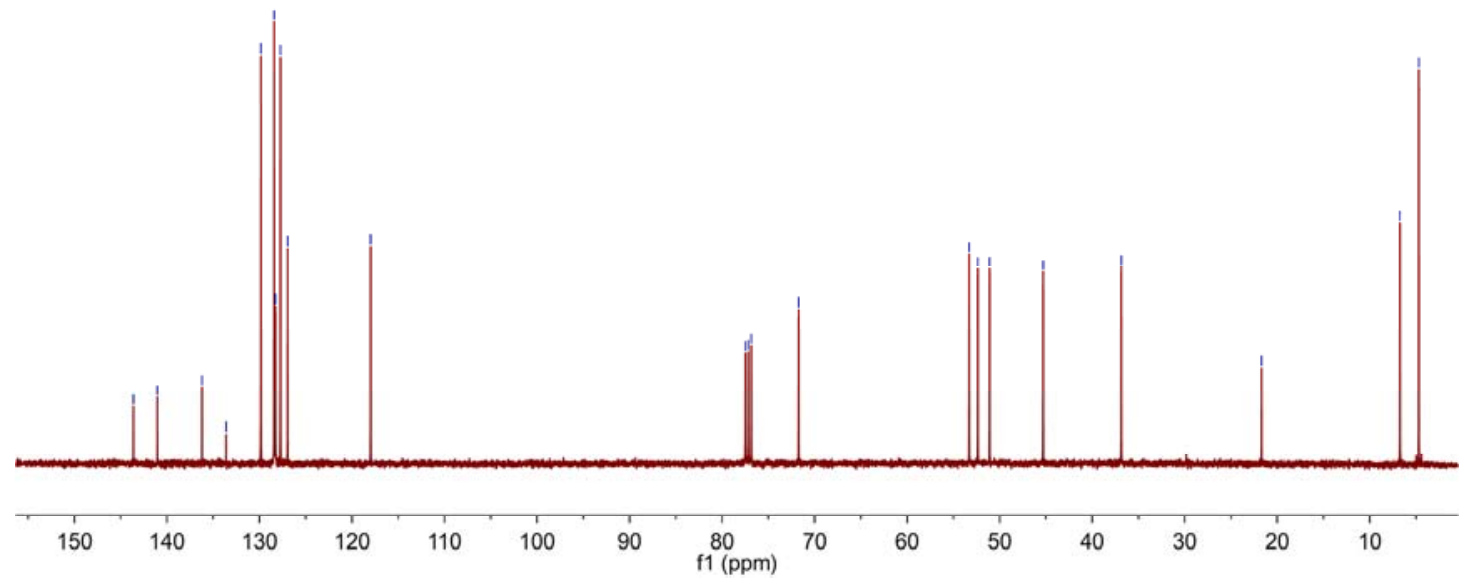



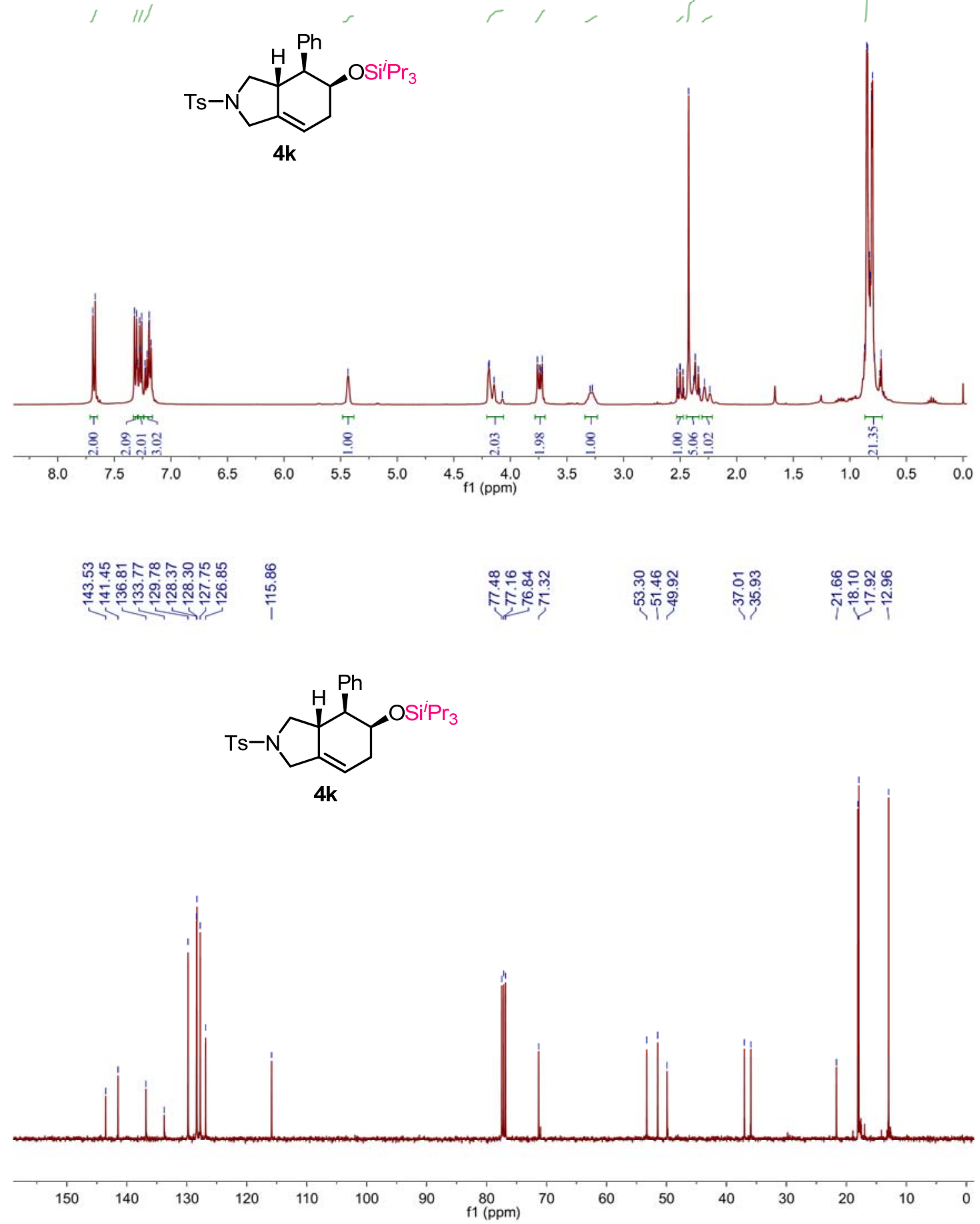
$1|1|$<smiles>CCO[C@H]1CC=C2CN([AsH])C[C@@]2(c2ccc(Oc3ccccc3)cc2)C1</smiles>

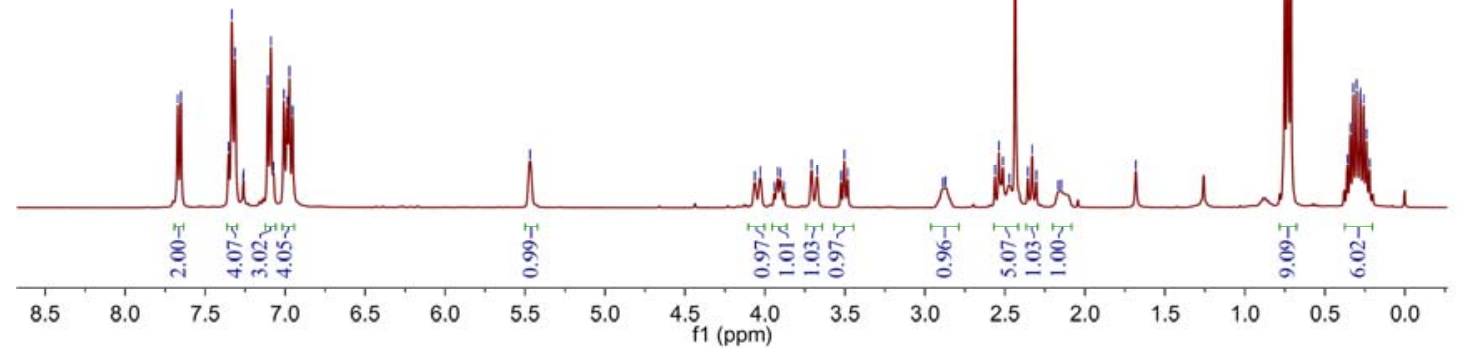

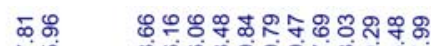

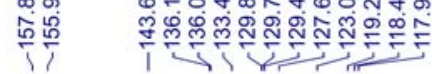<smiles>[3H]N1CC2=CC[C@H](OCC)[C@H](c3ccc(Oc4ccccc4)cc3)[C@@H]2C1</smiles>

31

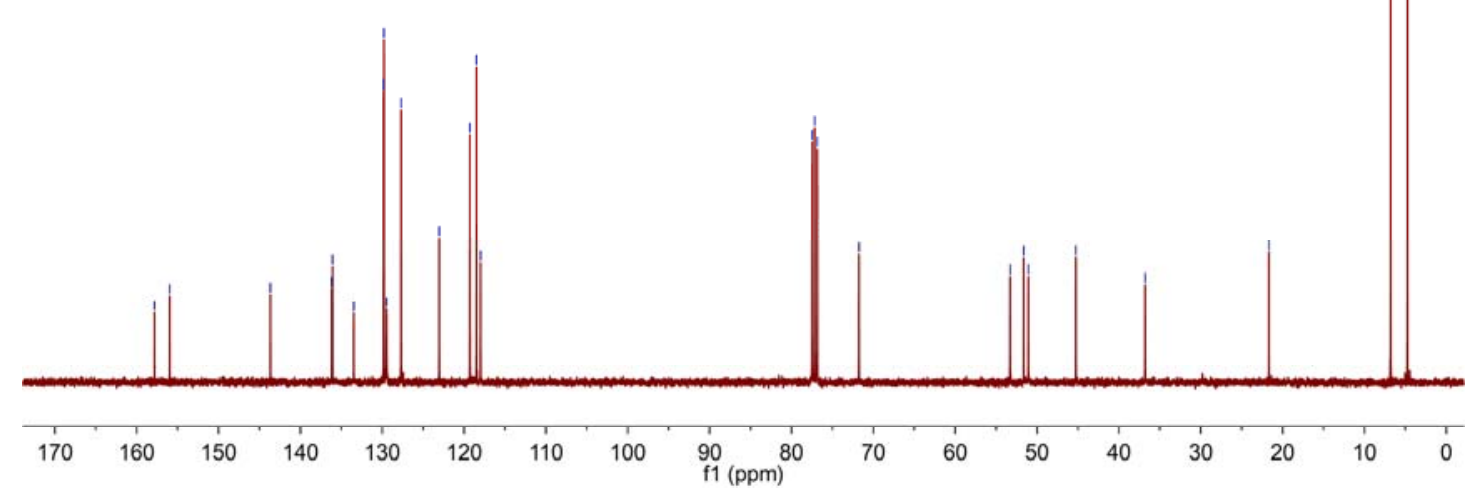



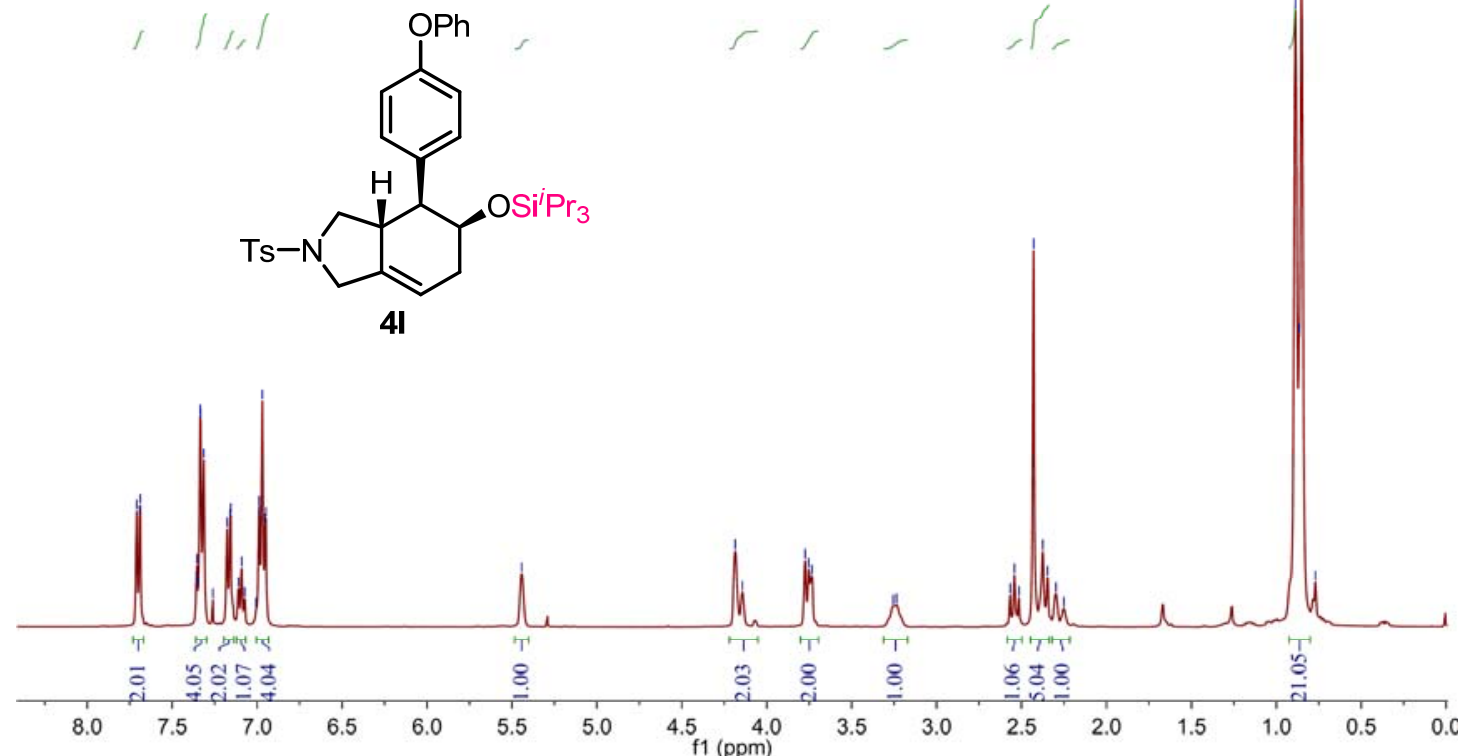

œ

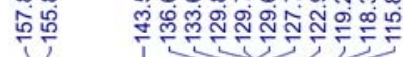

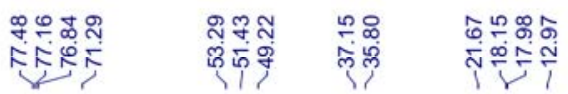

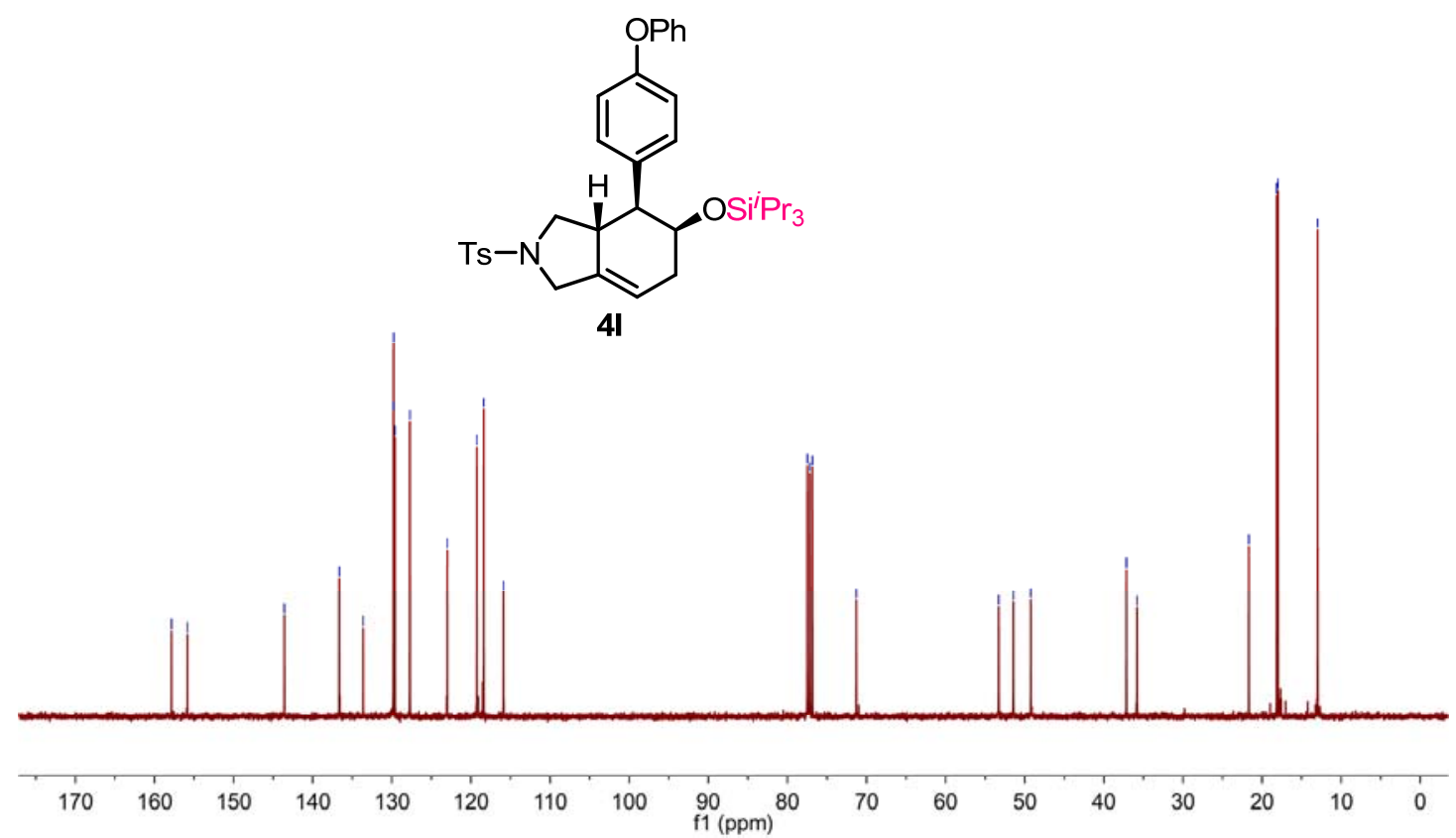



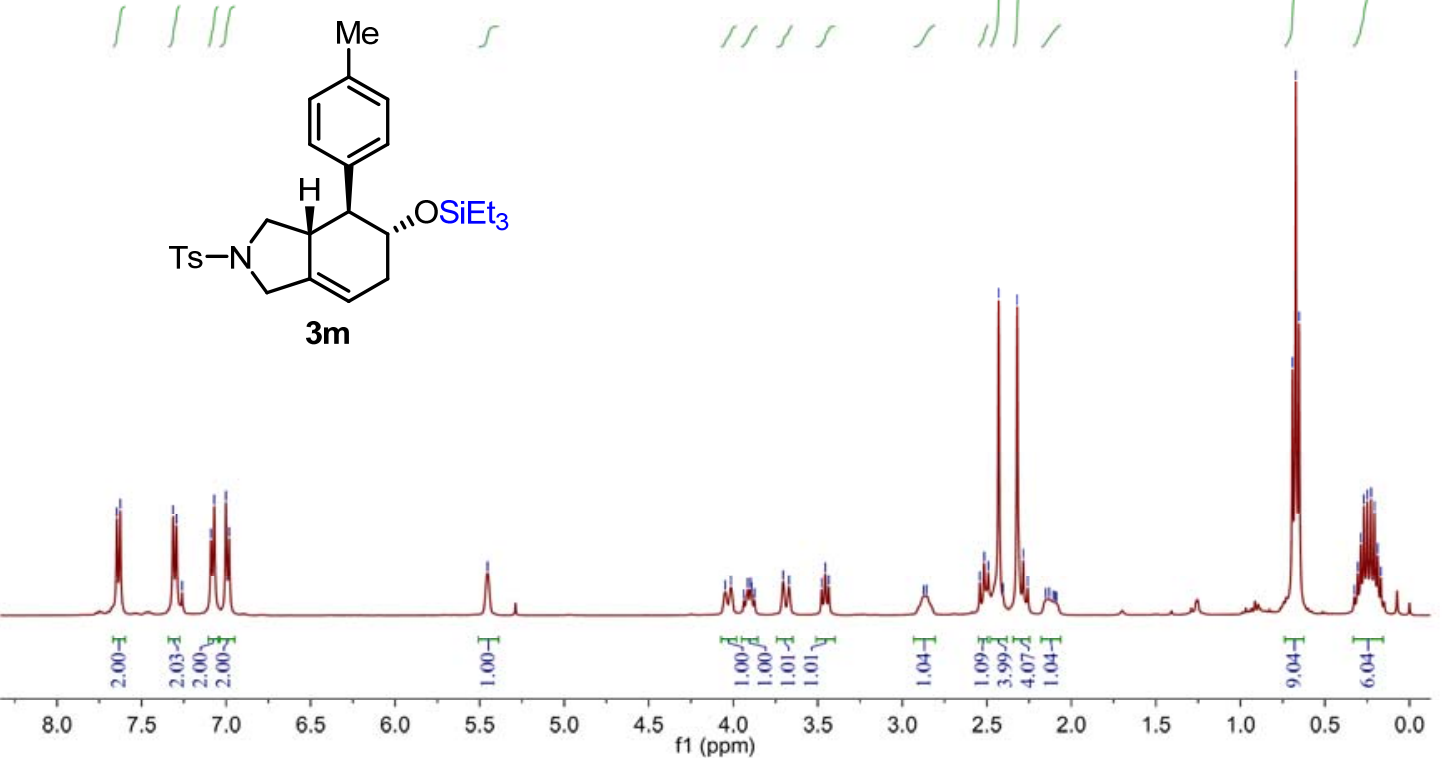

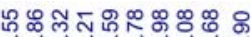

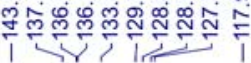

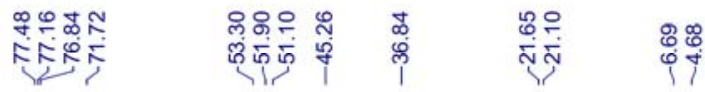<smiles>[3H]N1C[C@@H]2CC[C@H](OCC)[C@H](c3ccccc3)[C@]2(c2ccc(C)cc2)C1</smiles>

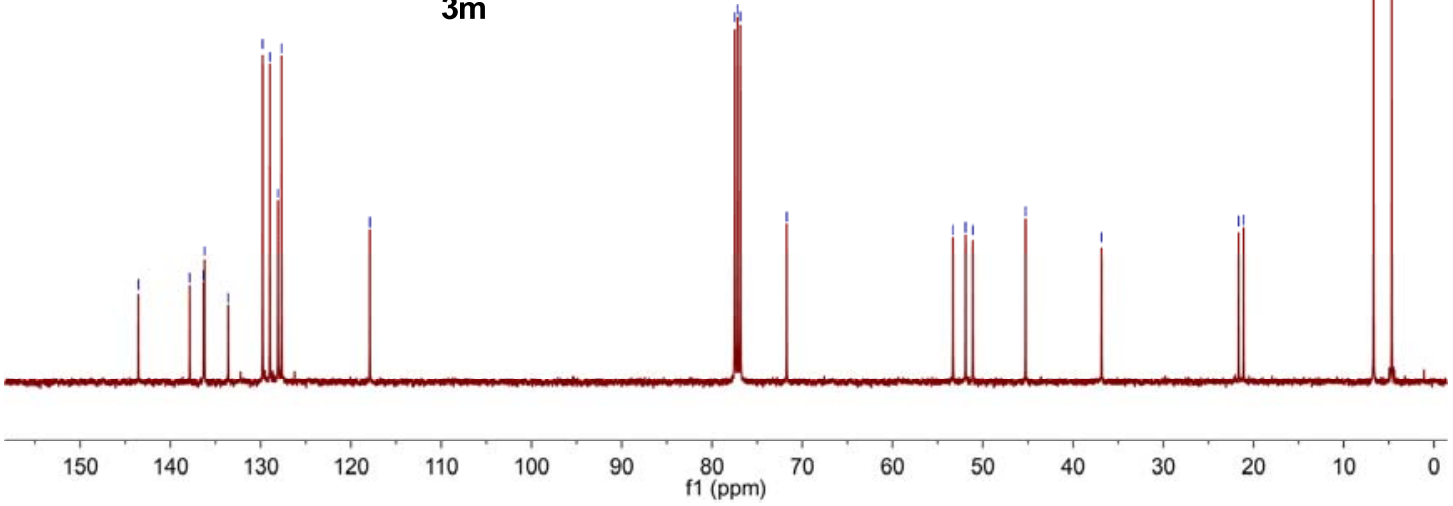




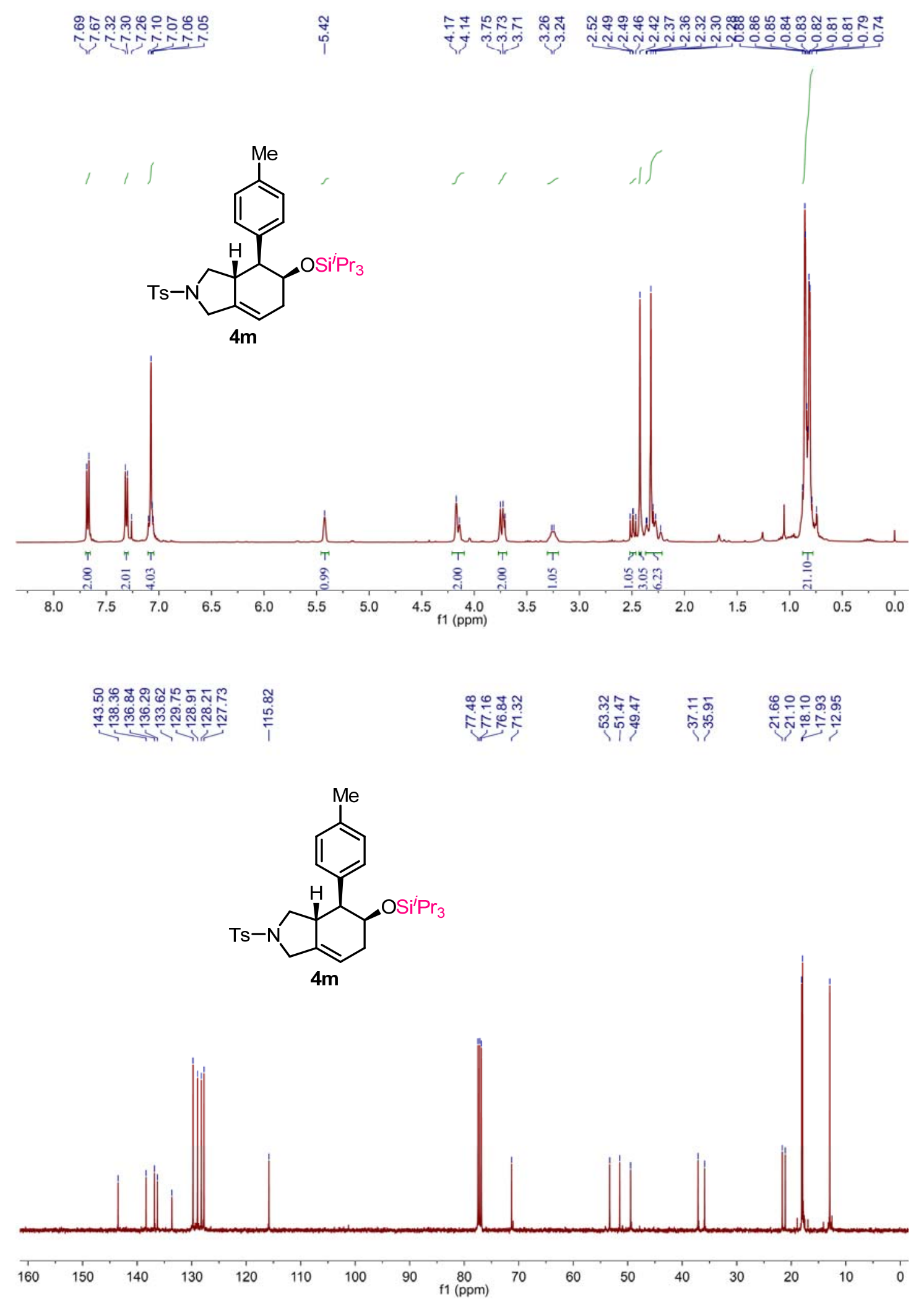




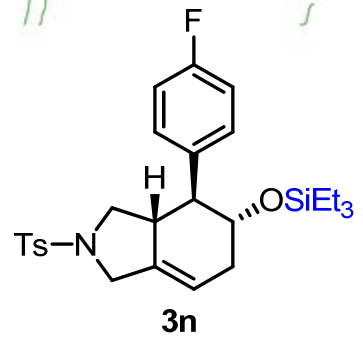
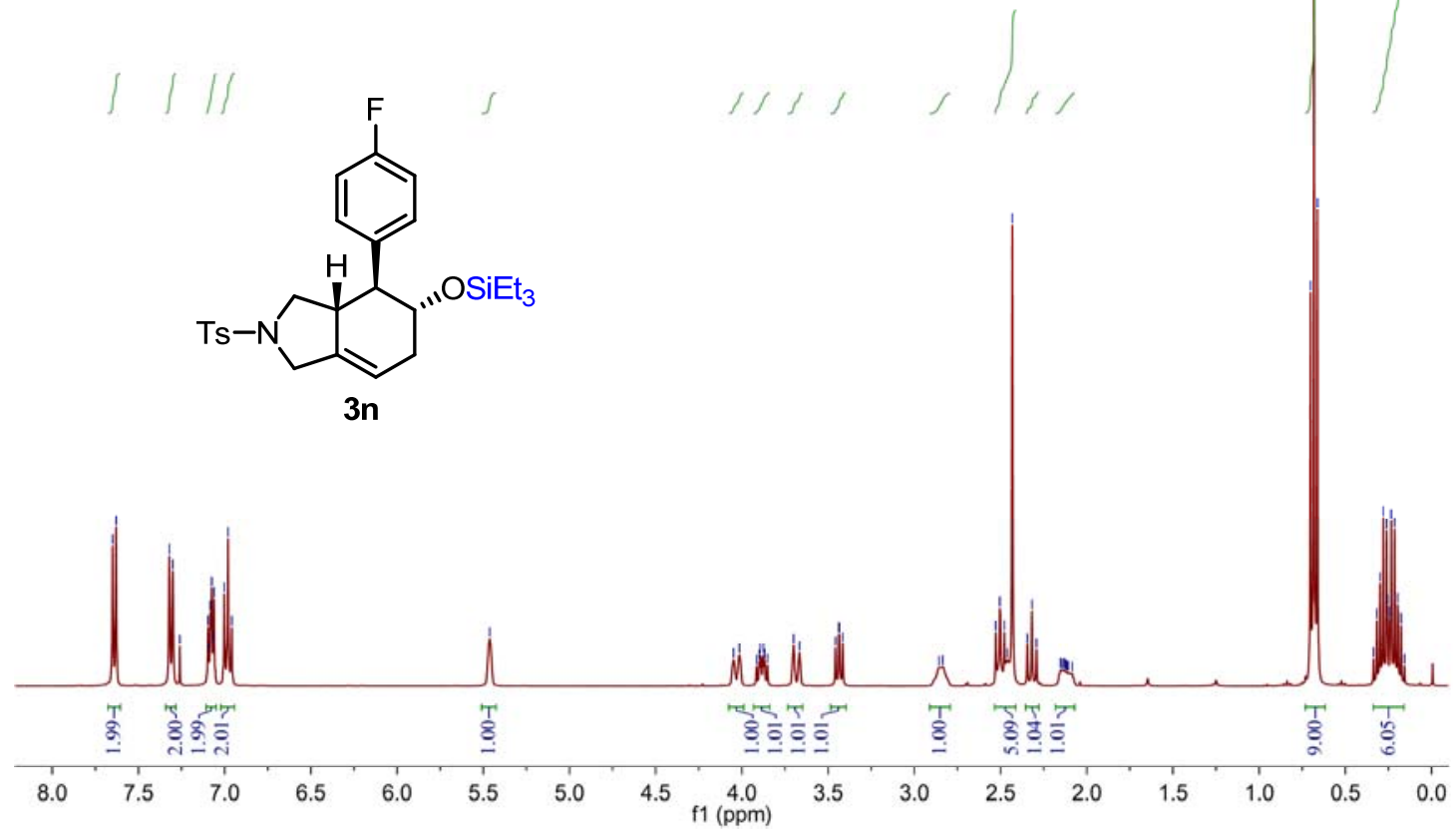

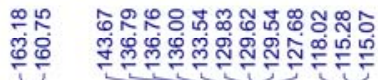

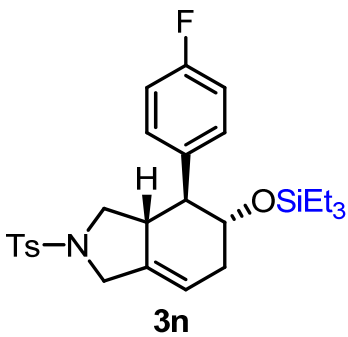

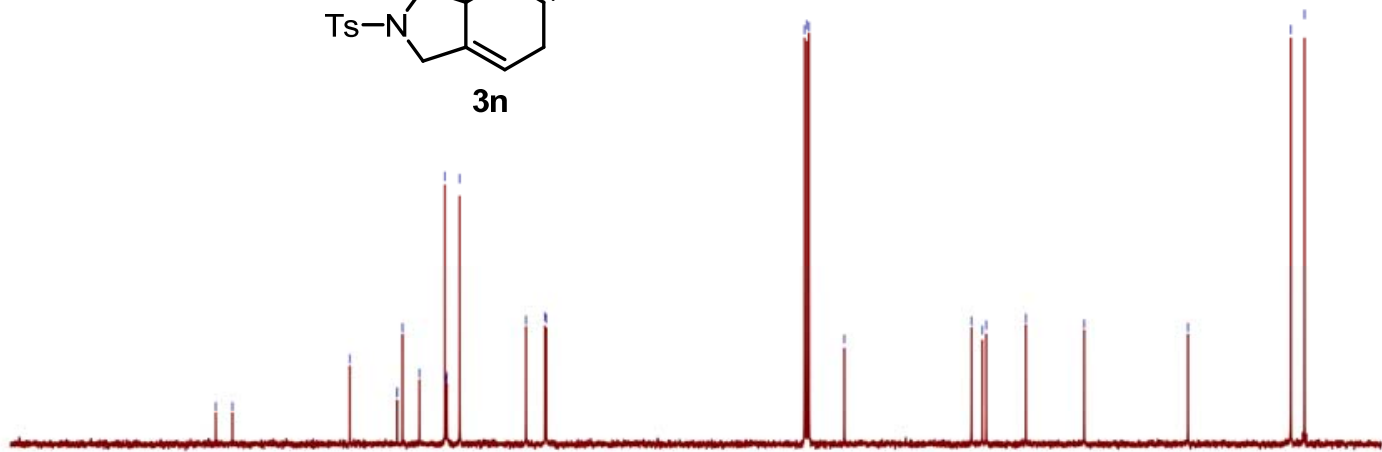

$\begin{array}{llllllllll}190 & 180 & 170 & 160 & 150 & 140 & 130 & 120 & 110 & 100 \\ \mathrm{f} 1(\mathrm{ppm})\end{array}$ 

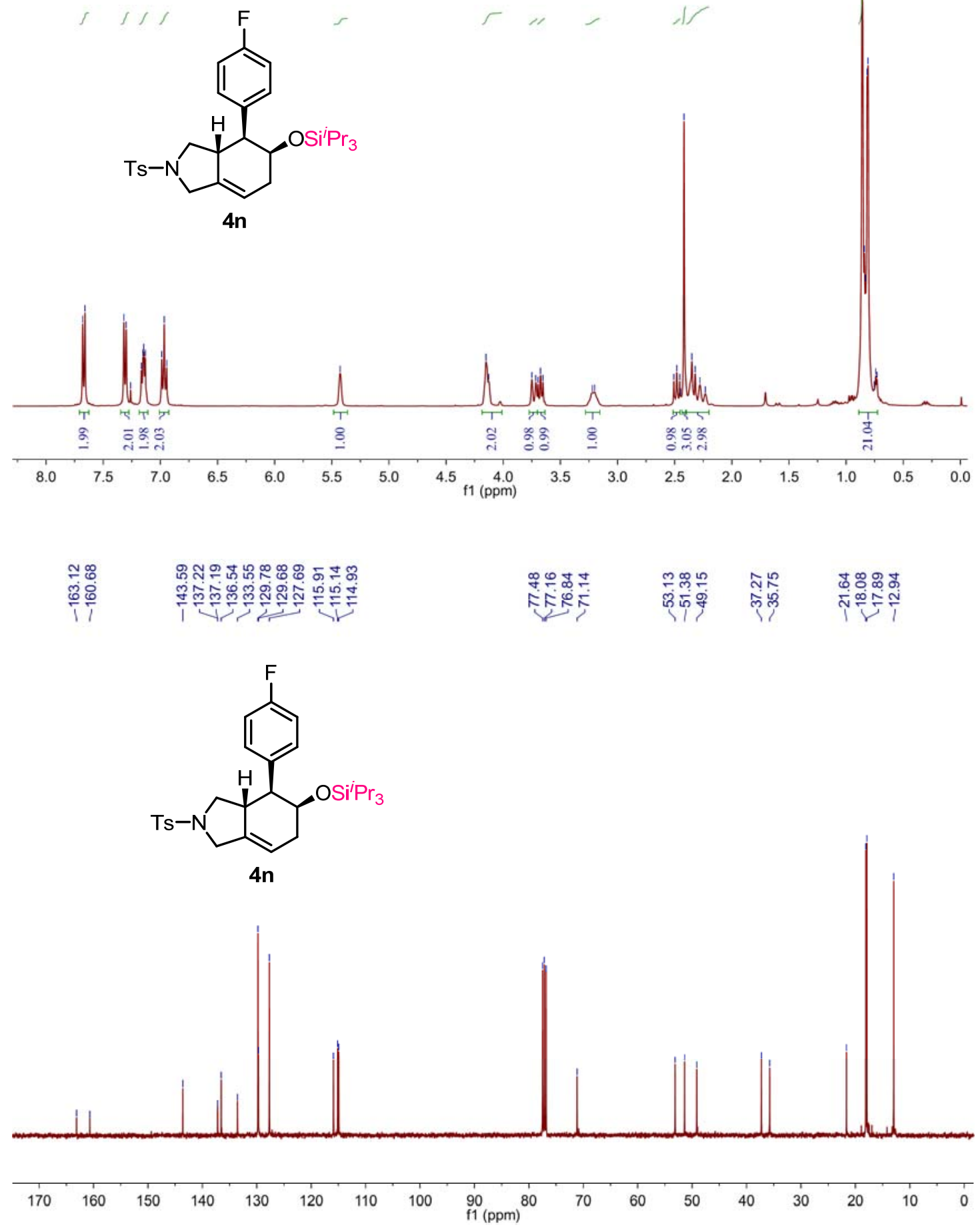

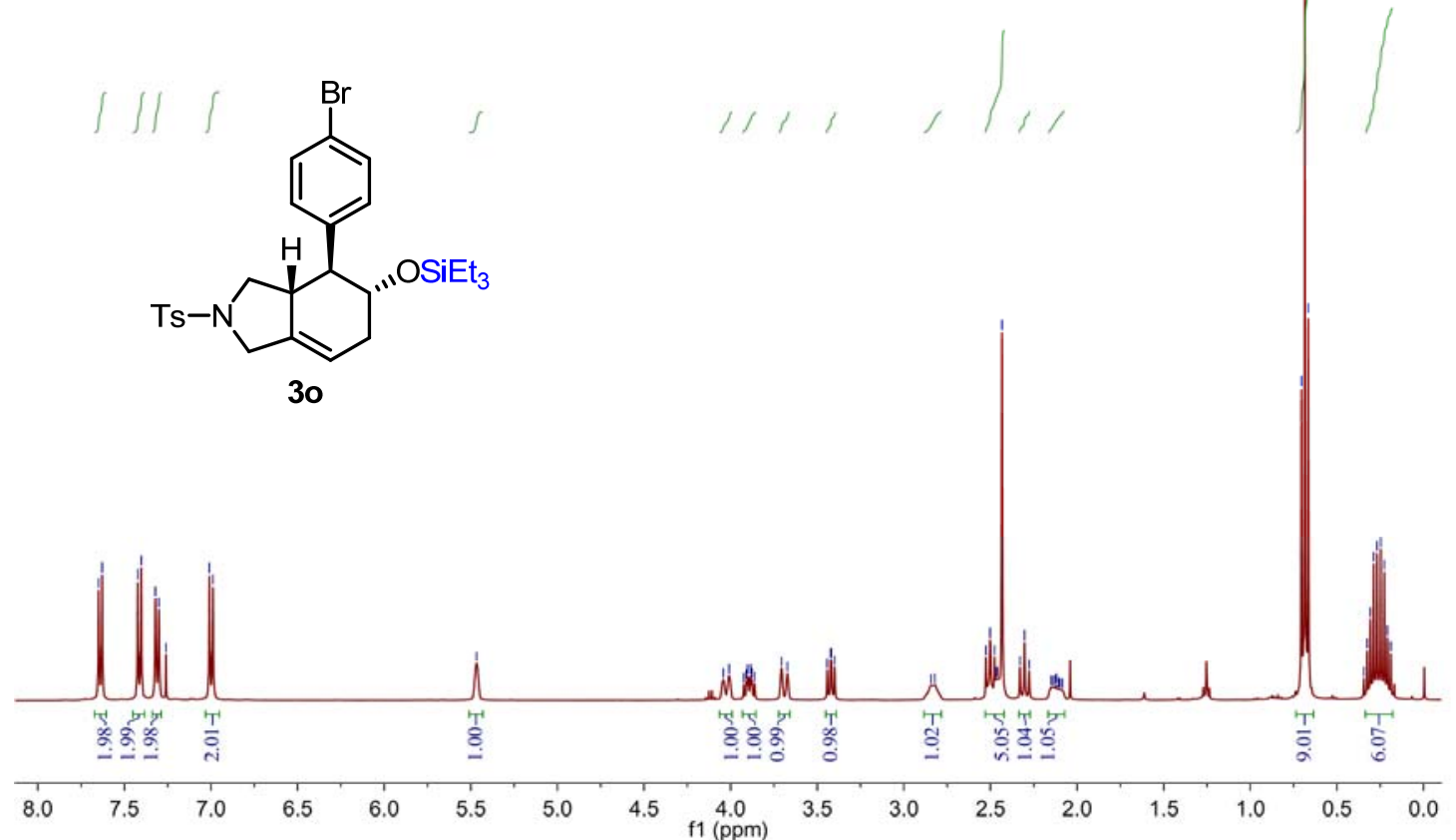

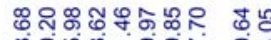

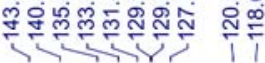

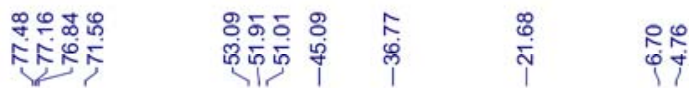<smiles>[3H]N1CC2=CC[C@H](OCC)C[C@]2(c2ccc(Br)cc2)C1</smiles>

30

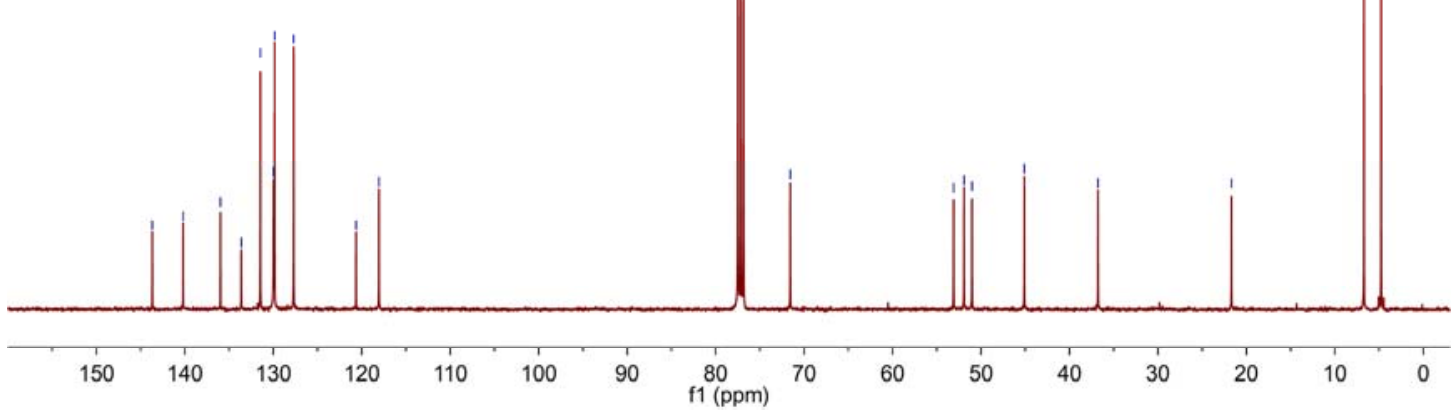



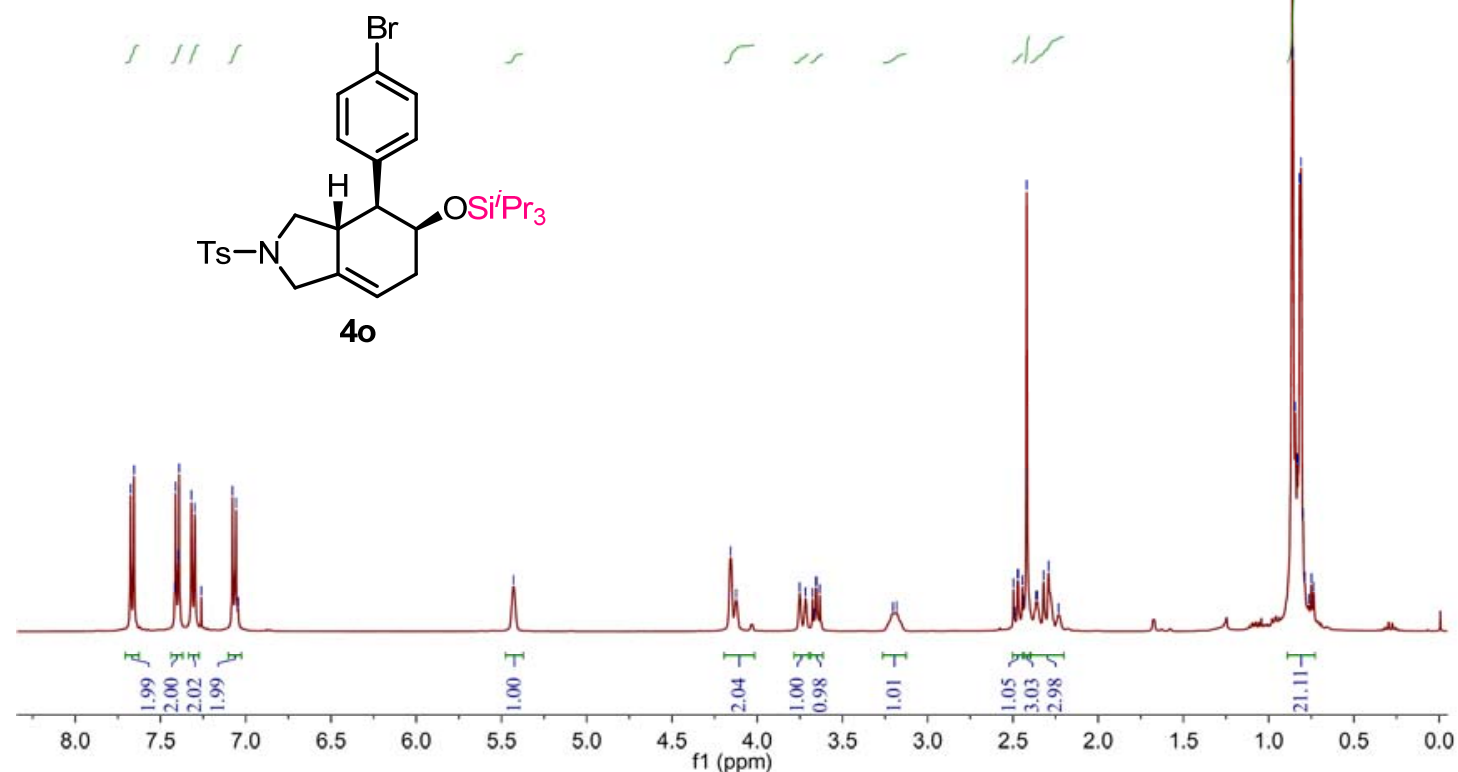

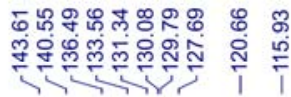

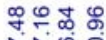

NR̂

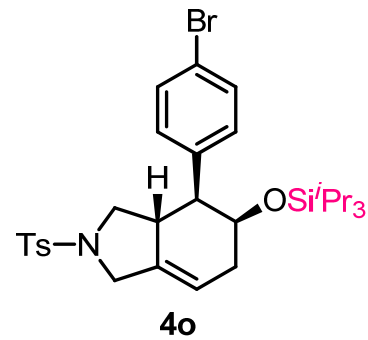

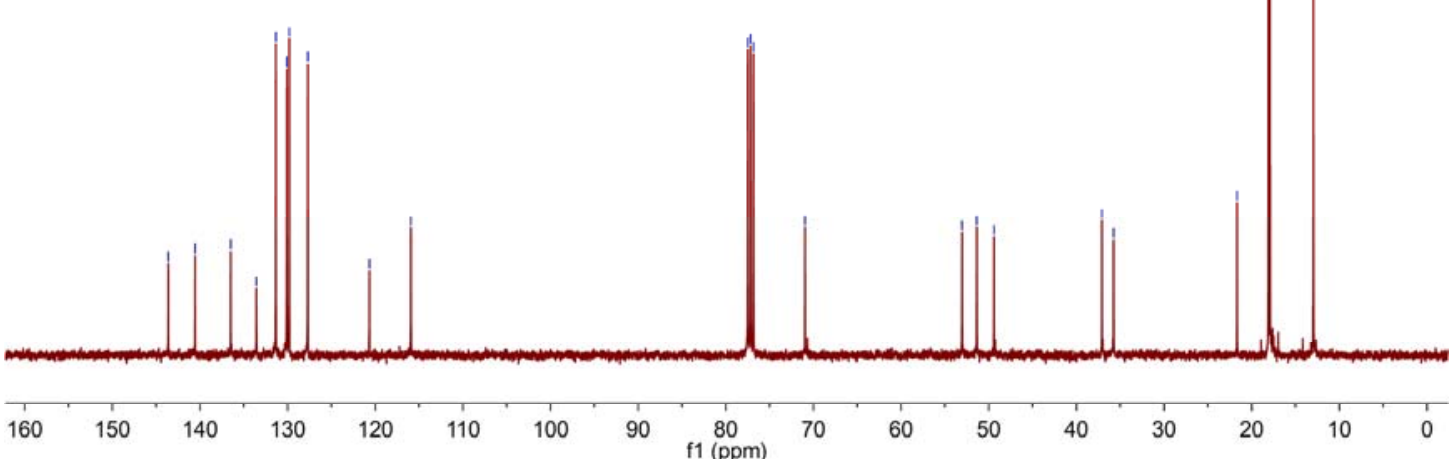


\|\|<smiles>CCO[C@H]1CC=C2CN([AsH])C[C@@H]2[C@H]1c1ccc(C(F)(F)F)cc1</smiles>

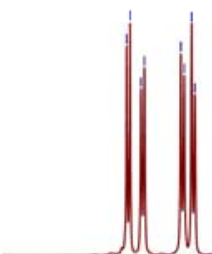

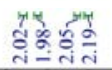

8.0

$7.5 \quad 7.0$
0

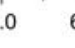

6.56 .0

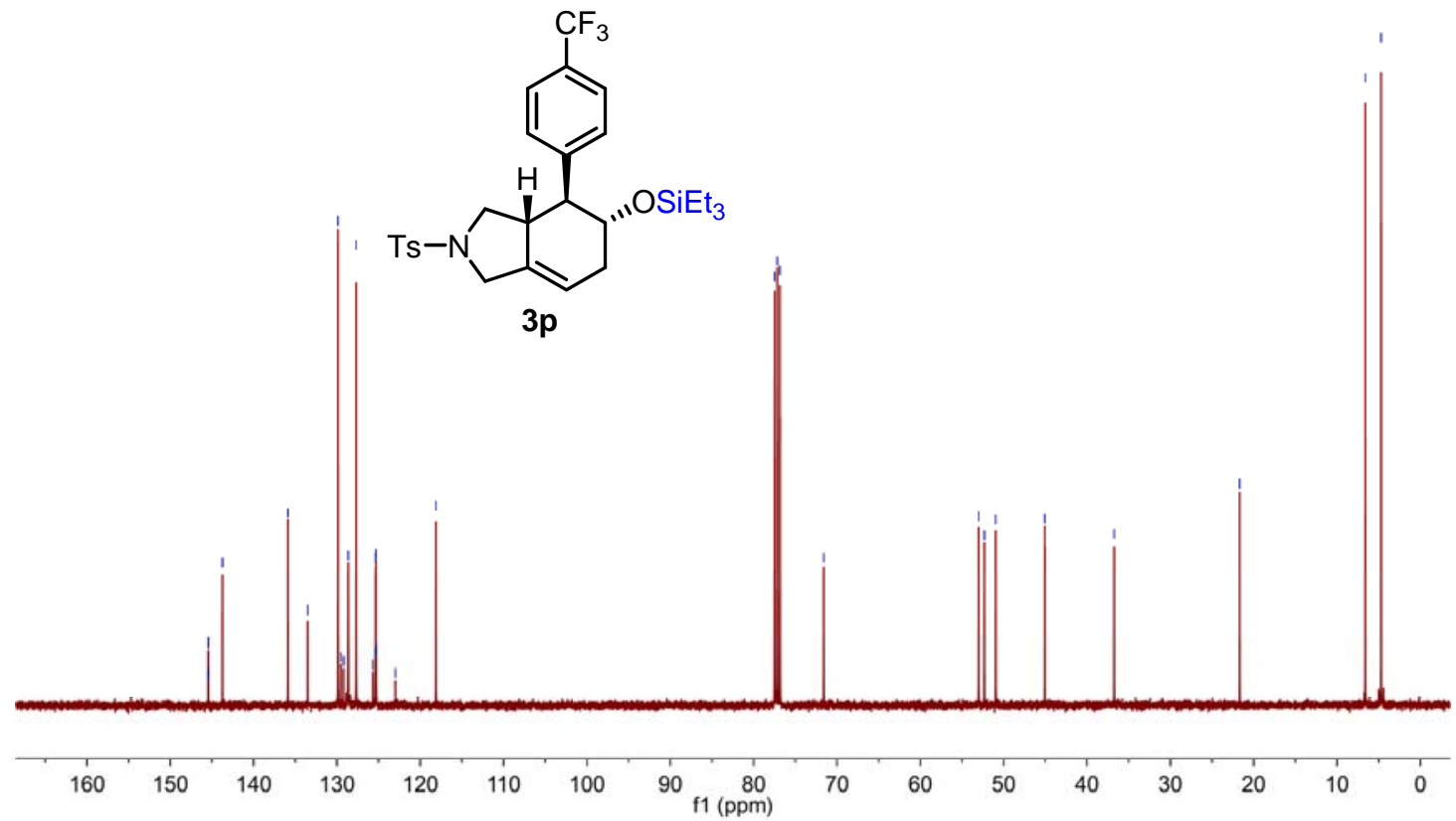




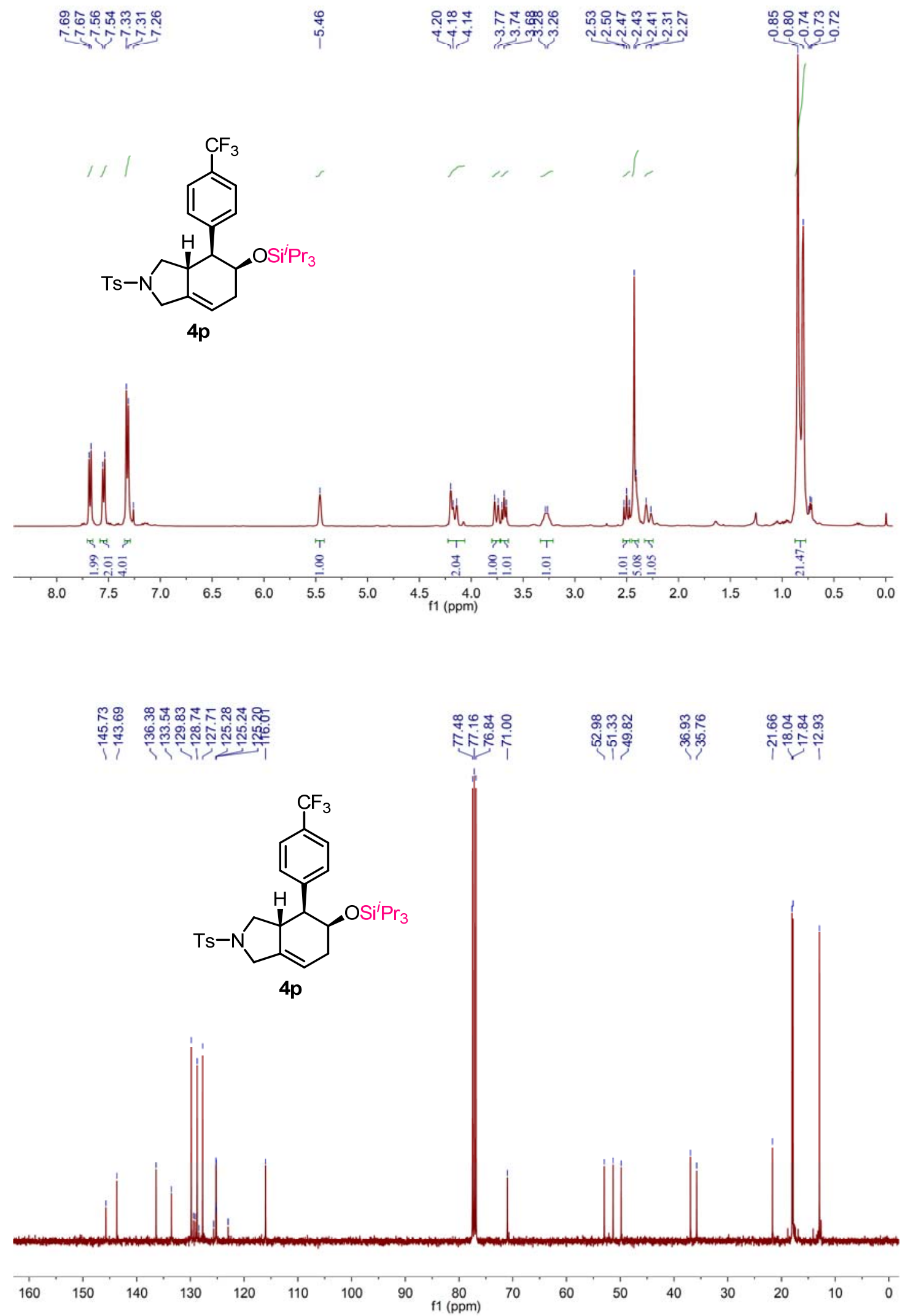




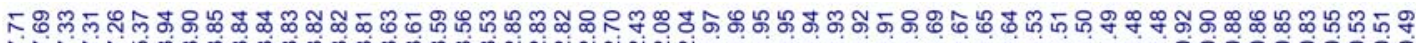

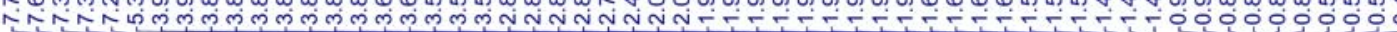<smiles>[3H]N1CC2=CC[C@H](OCC)C(CC)[C@H]2C[C@H]1[Hg]</smiles>

$3 q$

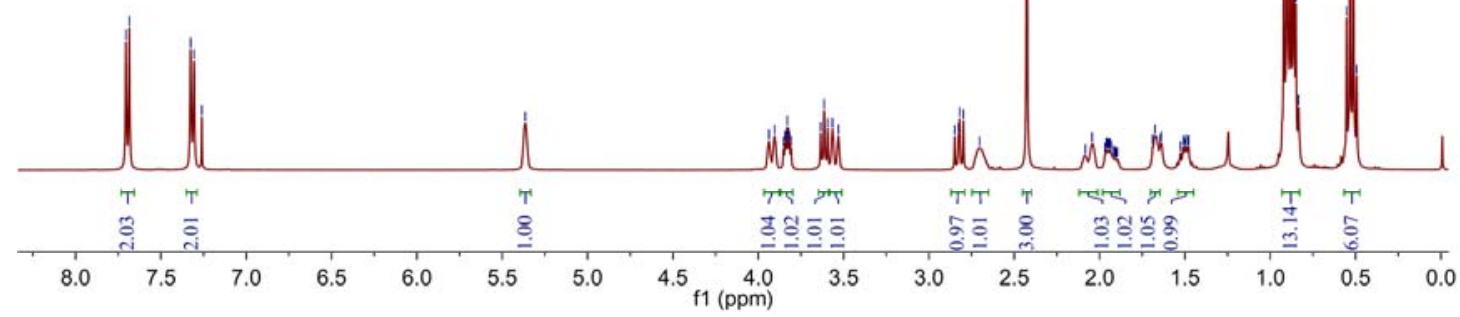

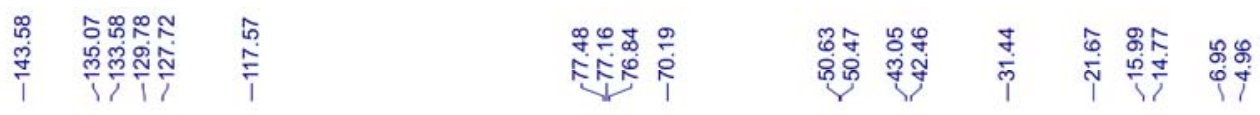<smiles>[B]N1CC2=CC[C@H](OCC)[C@H](CC)[C@H]2C1</smiles>

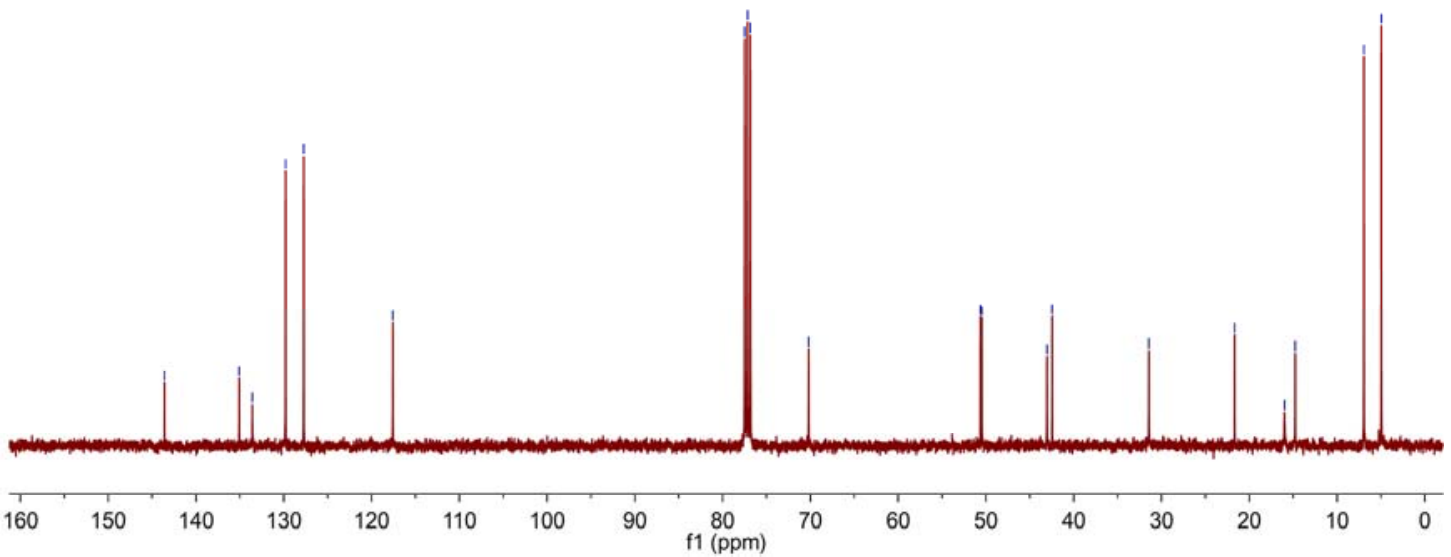




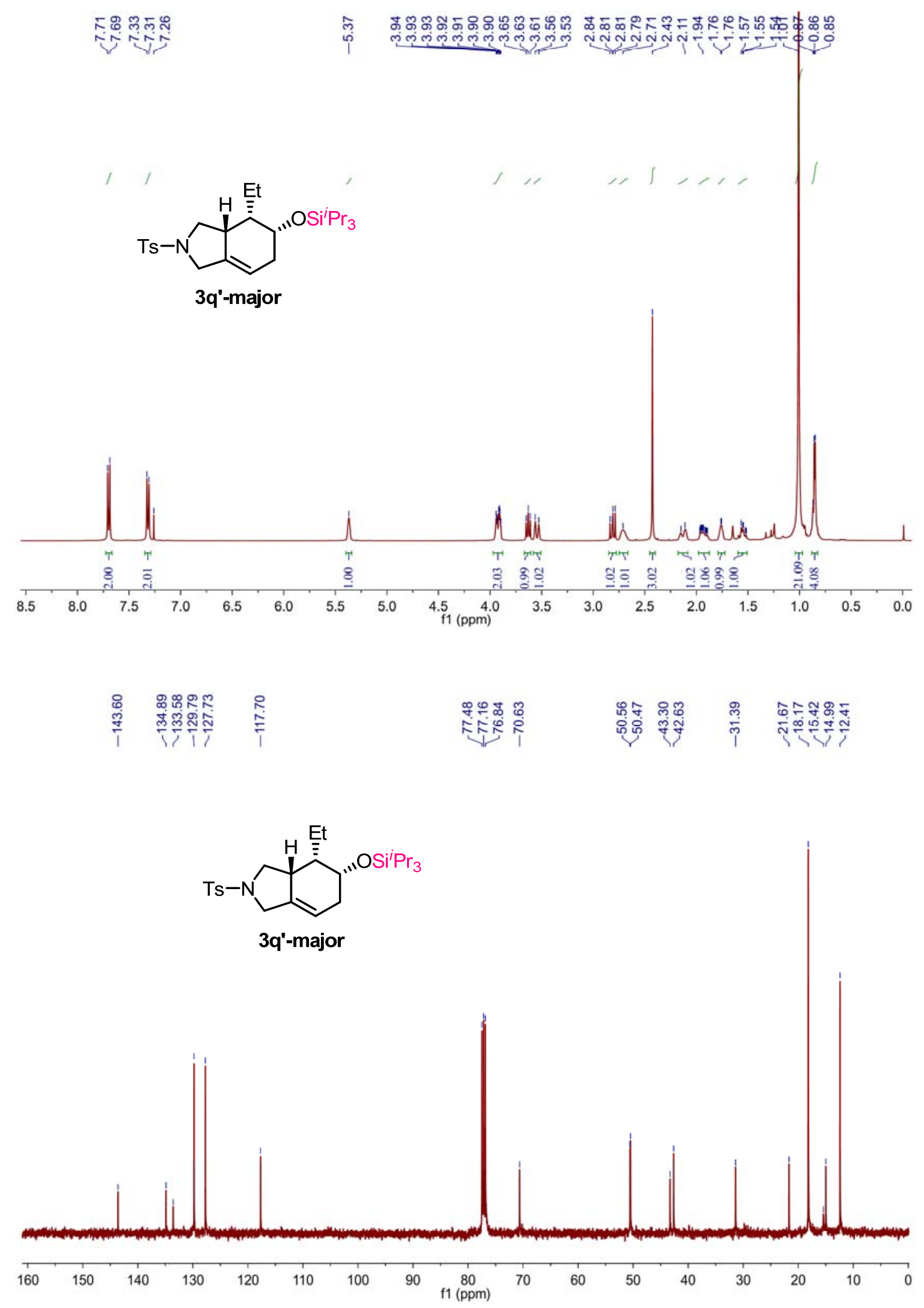



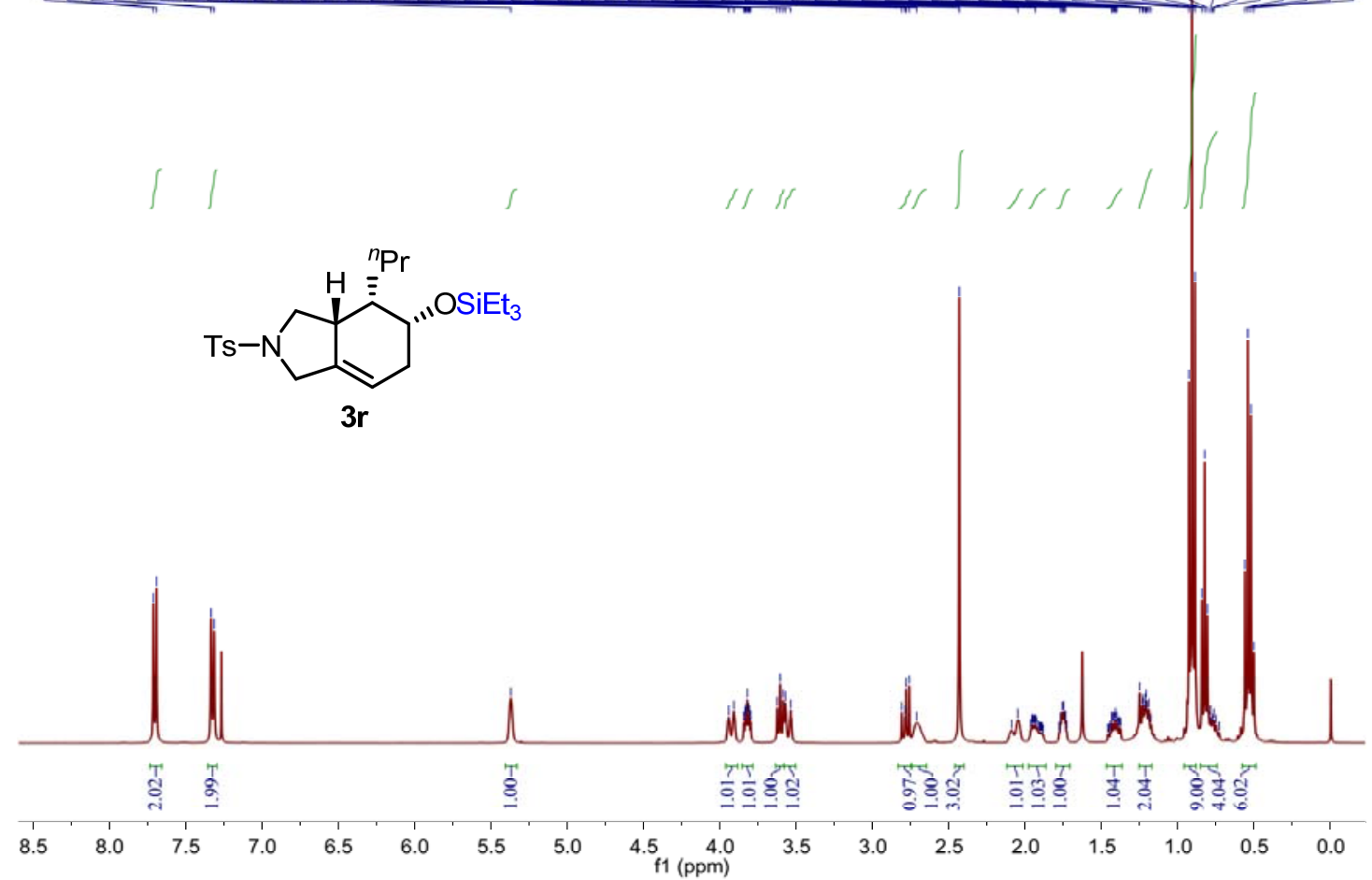<smiles>[3H]N1CC2=CC[C@H](OCC)[C@H](C(C)C)[C@@H]2C1</smiles>
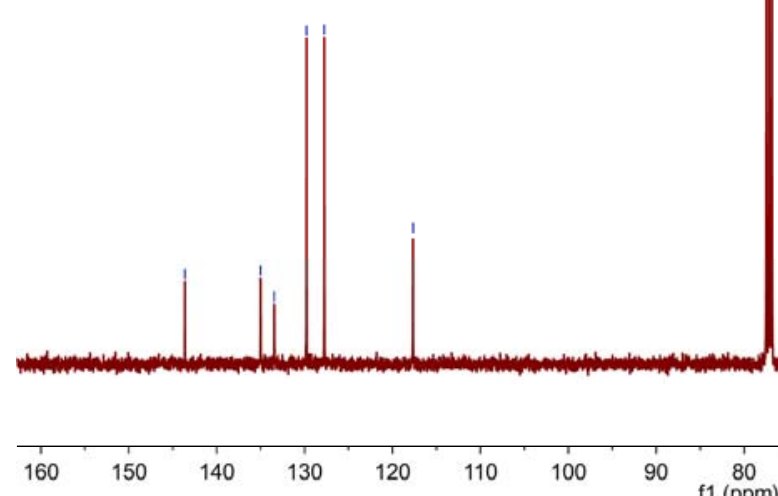

90 


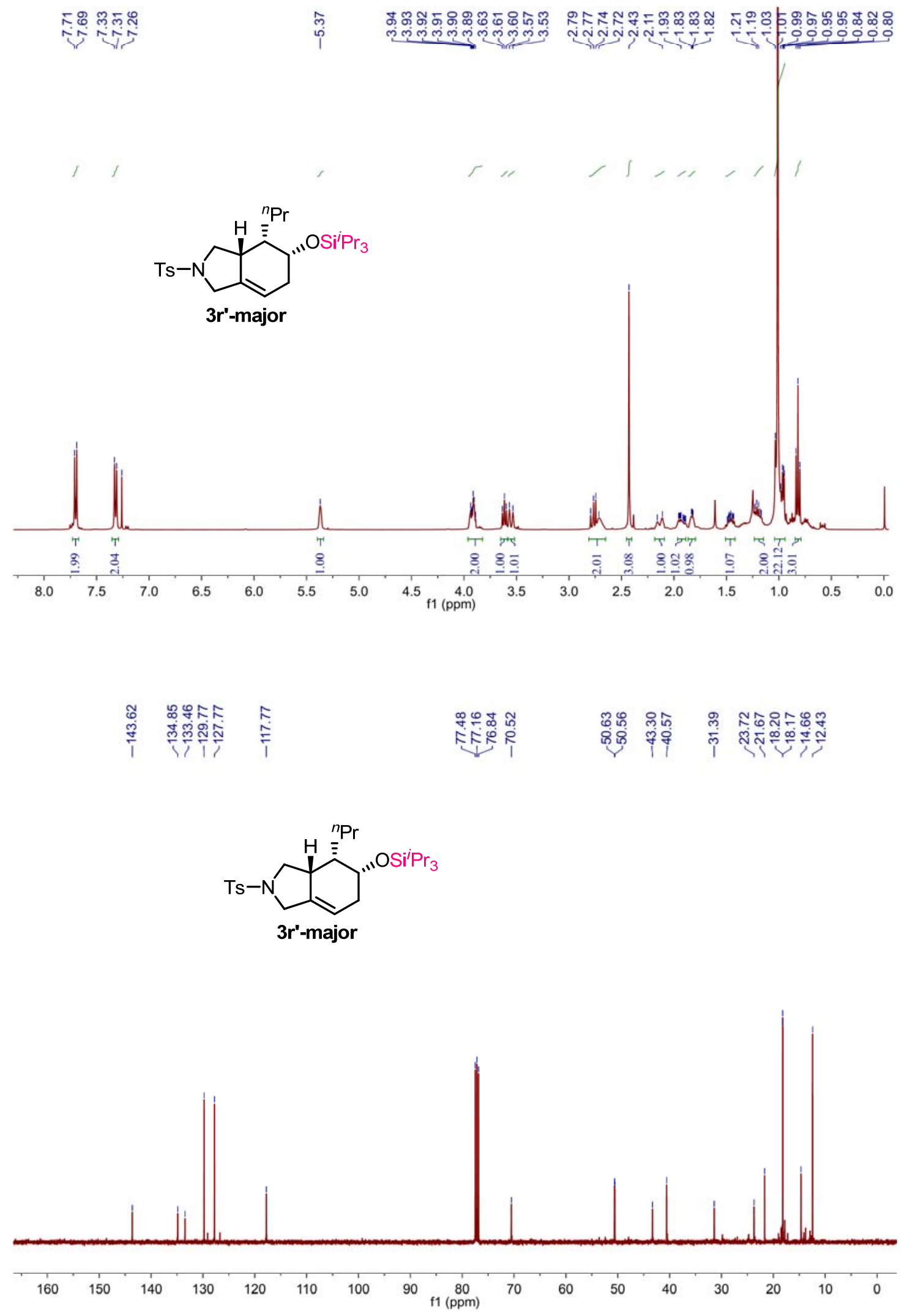




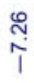

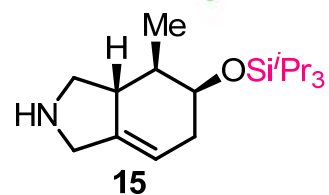

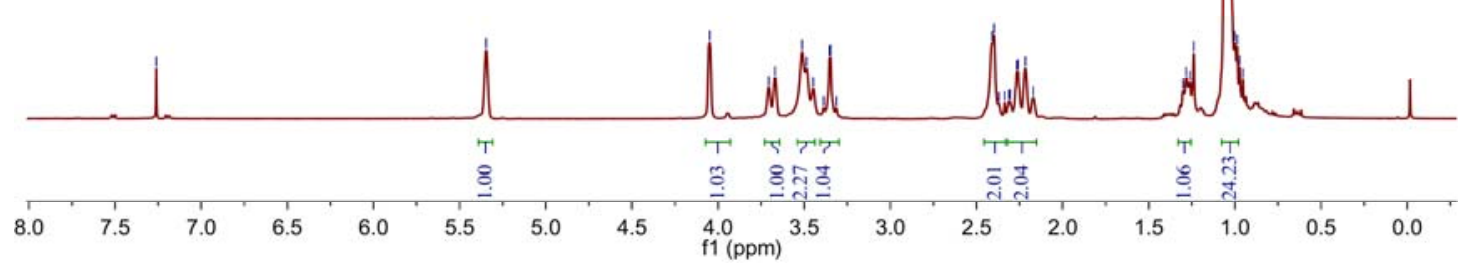

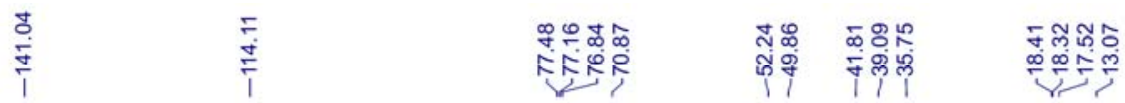<smiles>CCC[SiH2]O[C@H]1CC=C2CNC[C@H]2C(C)[C@H]1C</smiles>

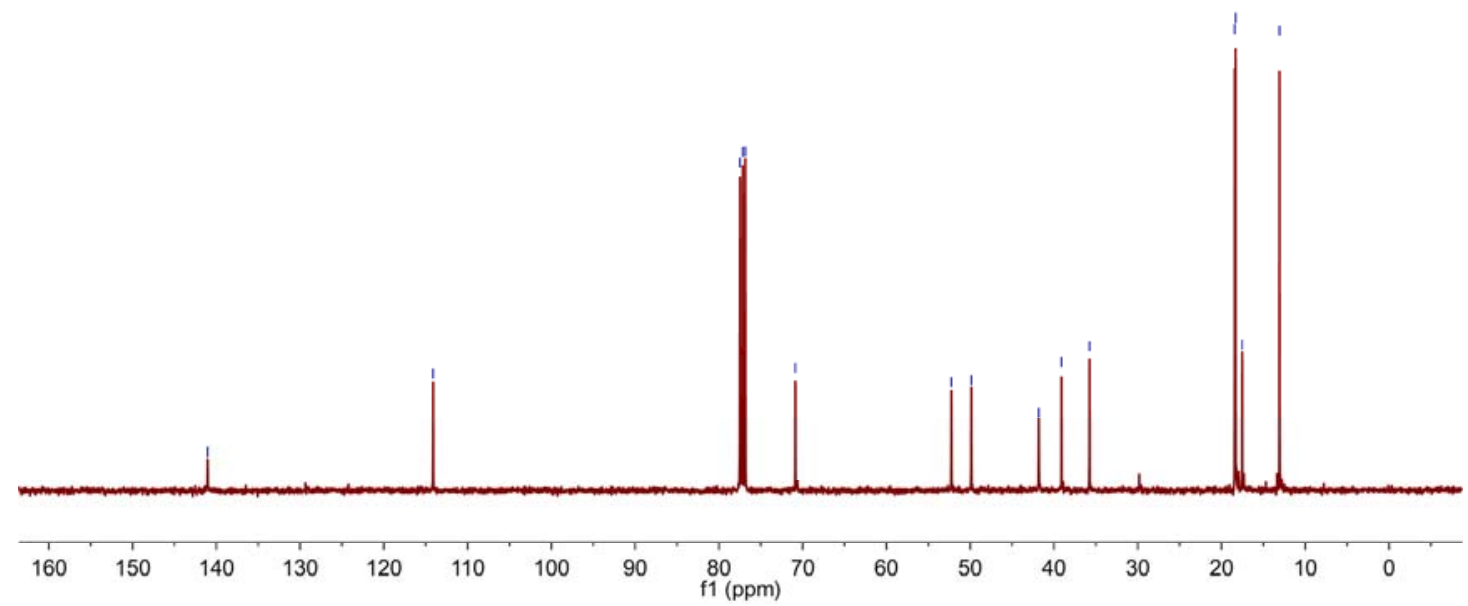




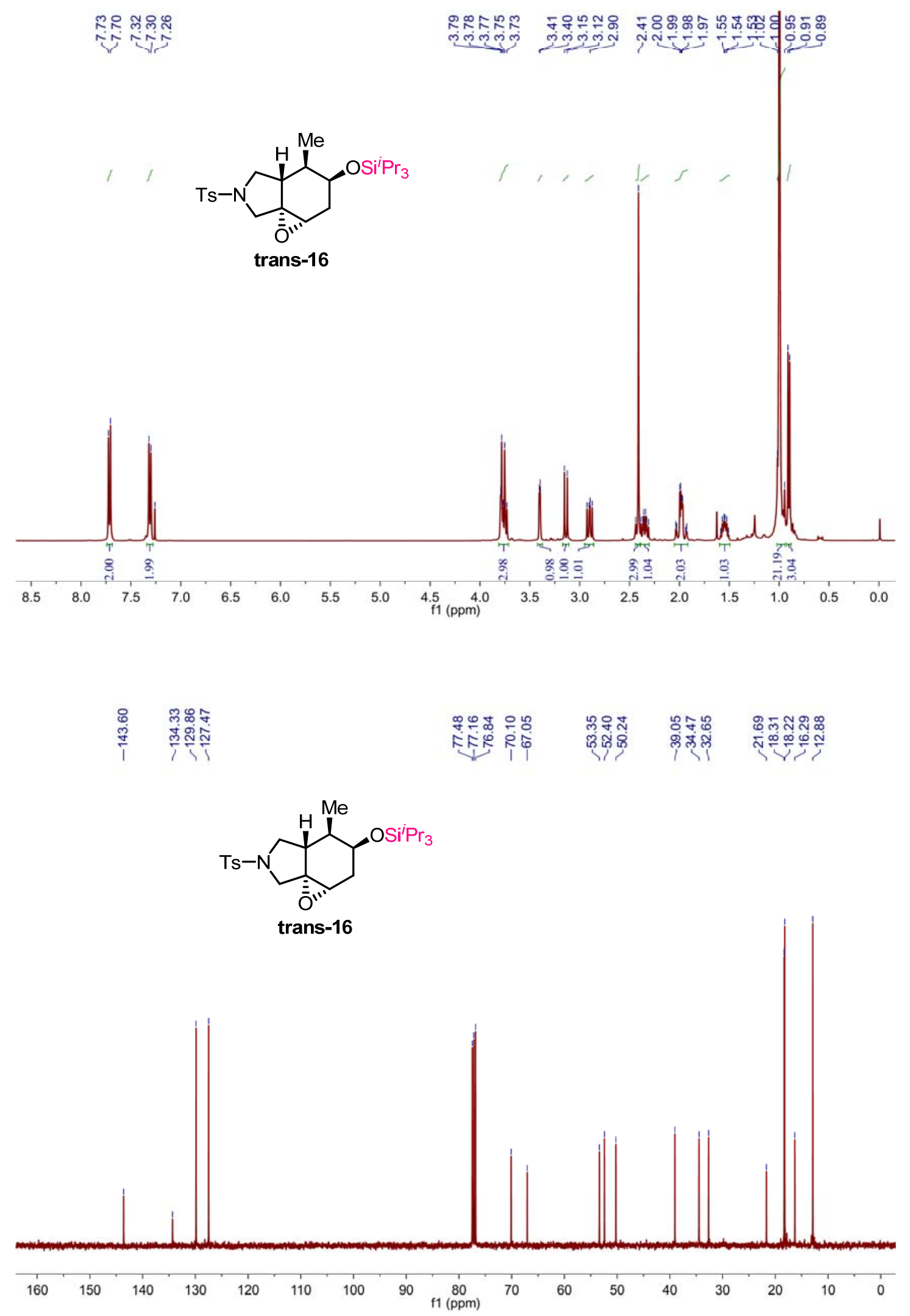



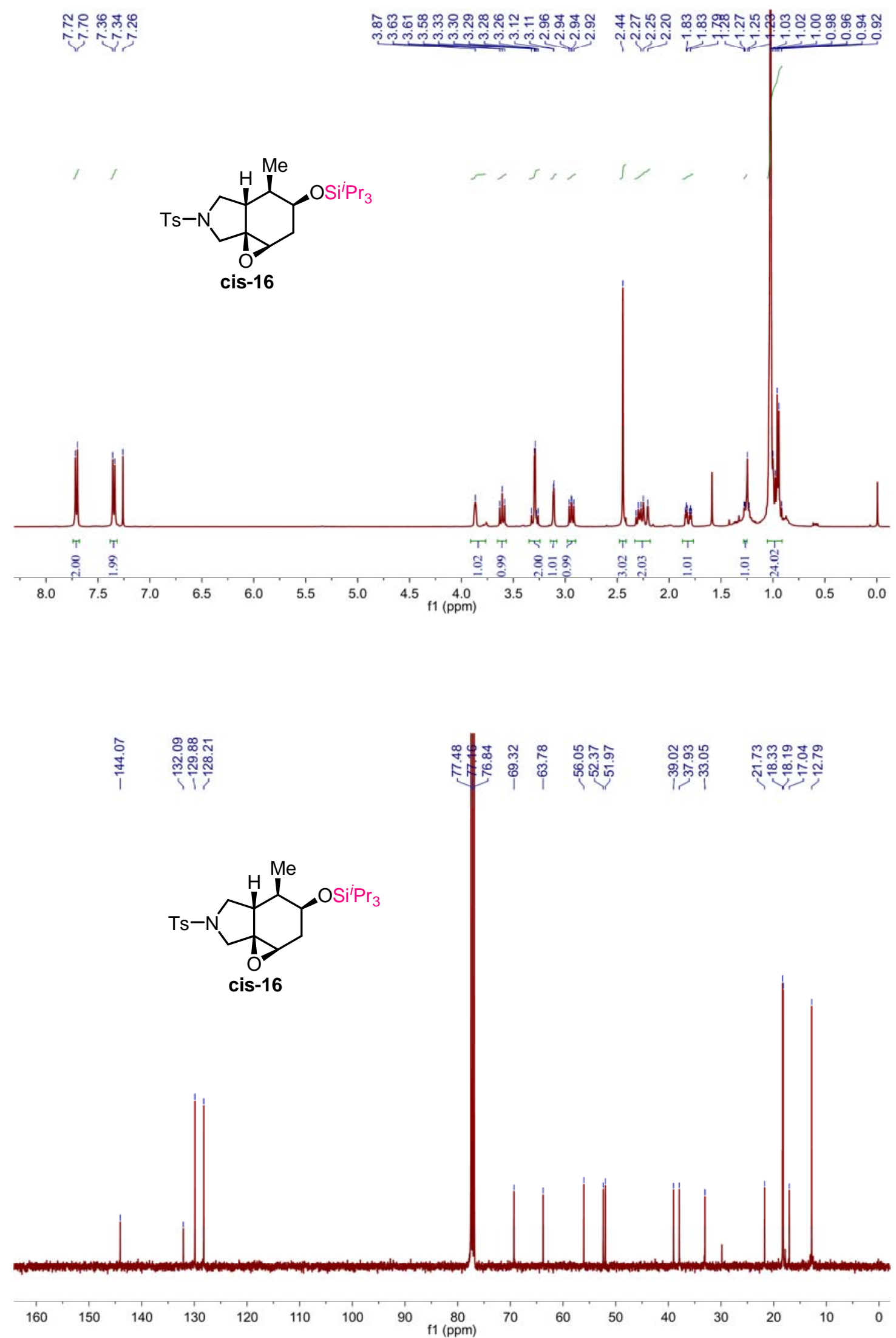

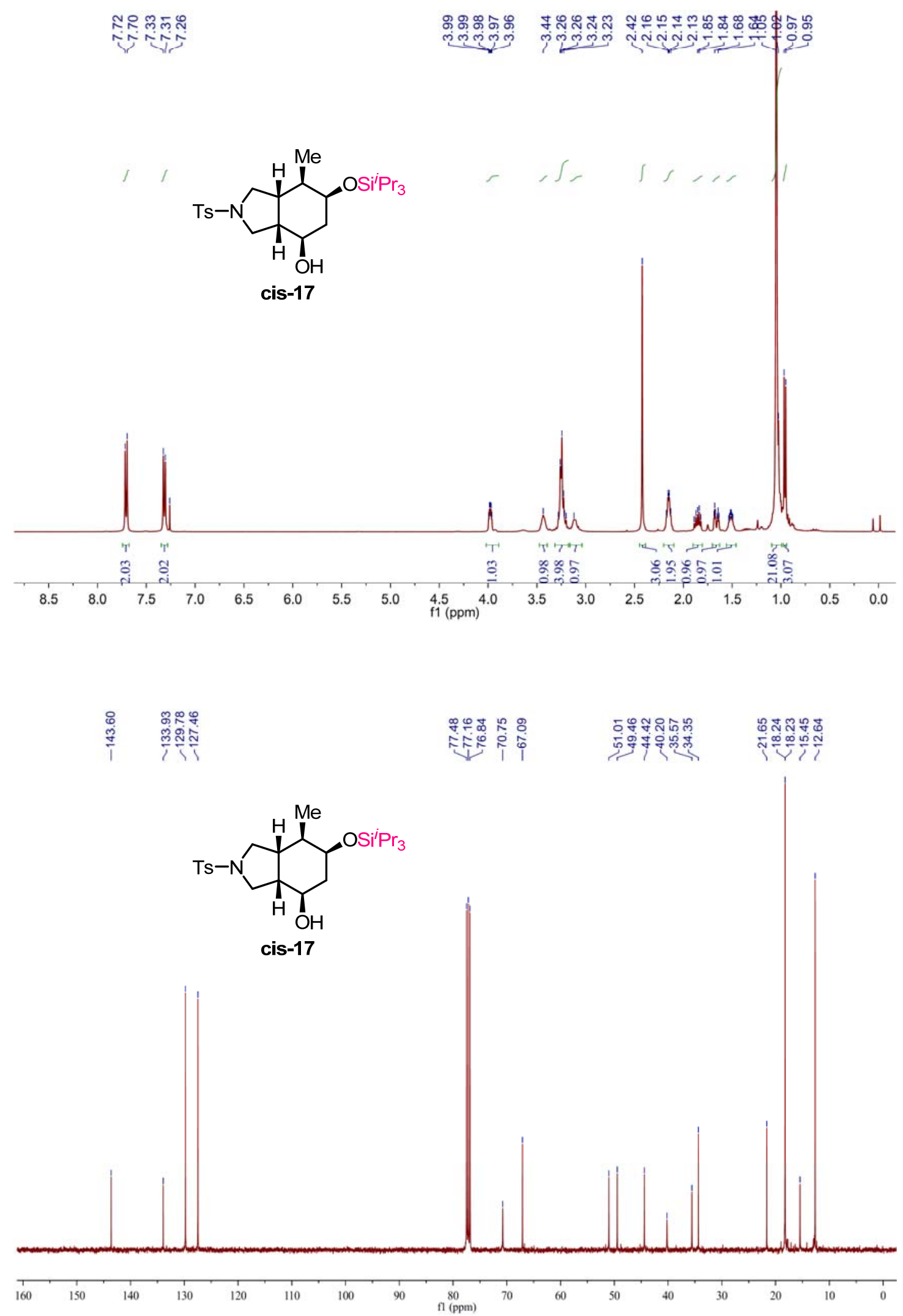


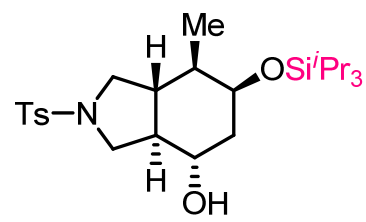

trans-17
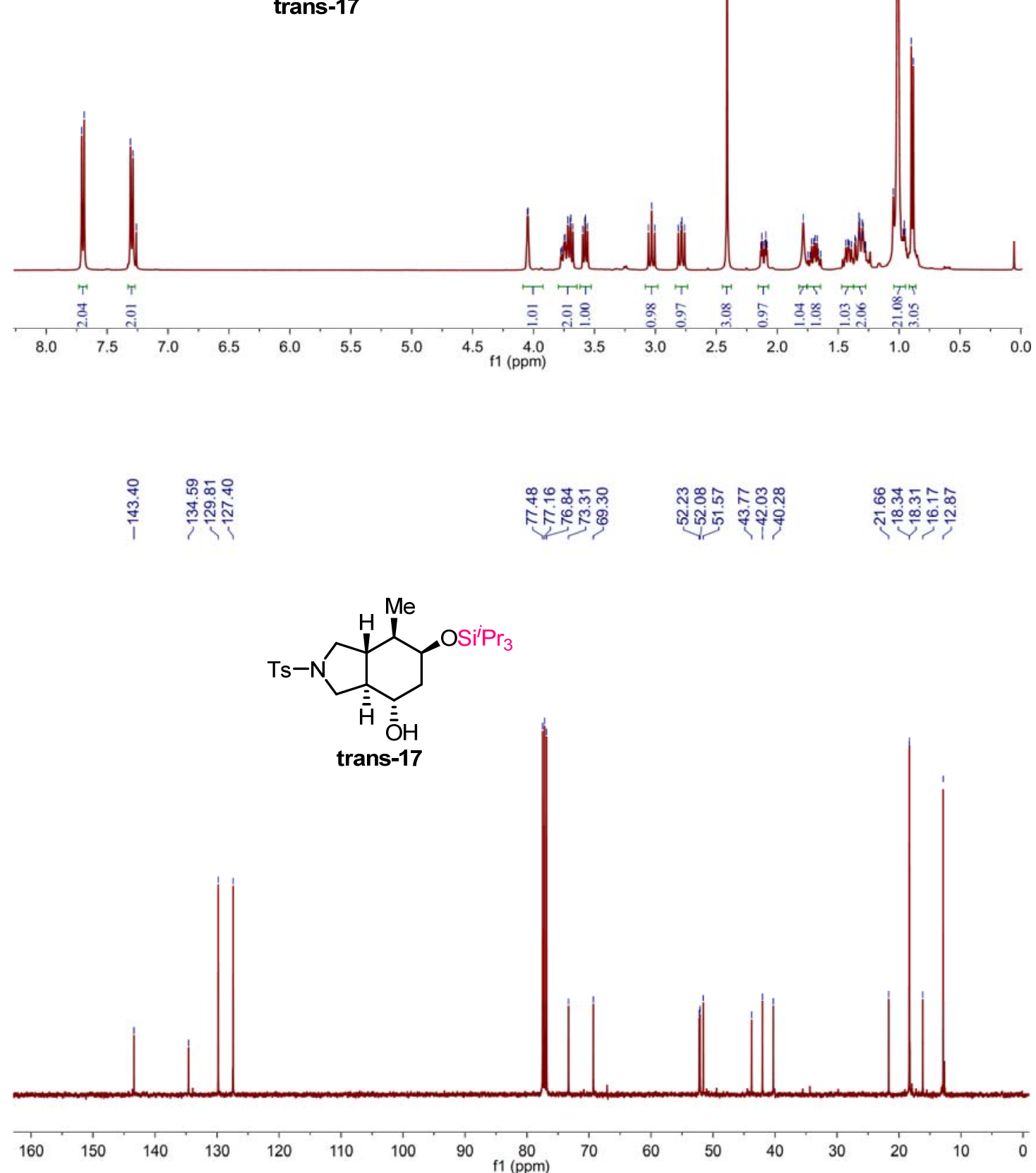


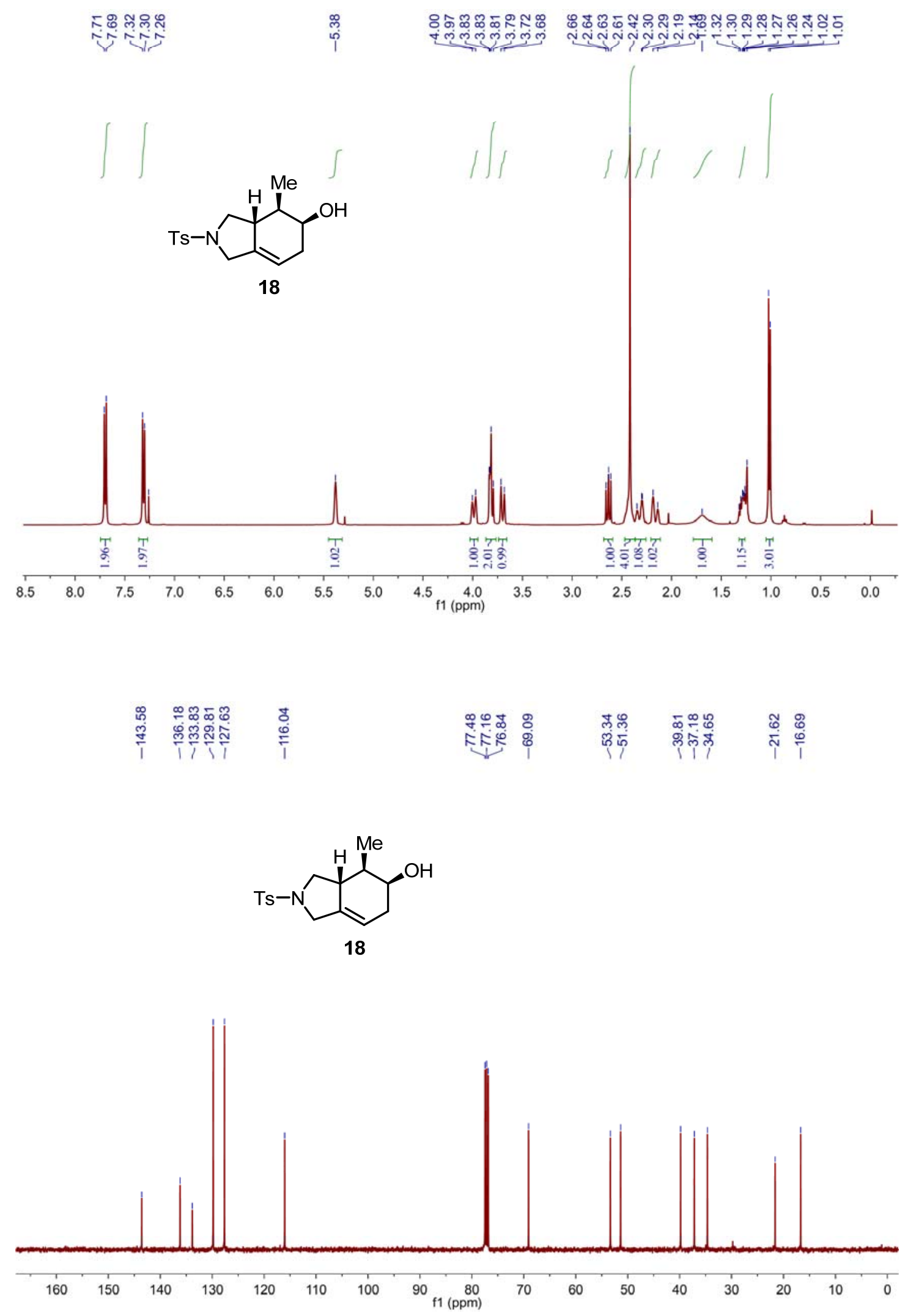




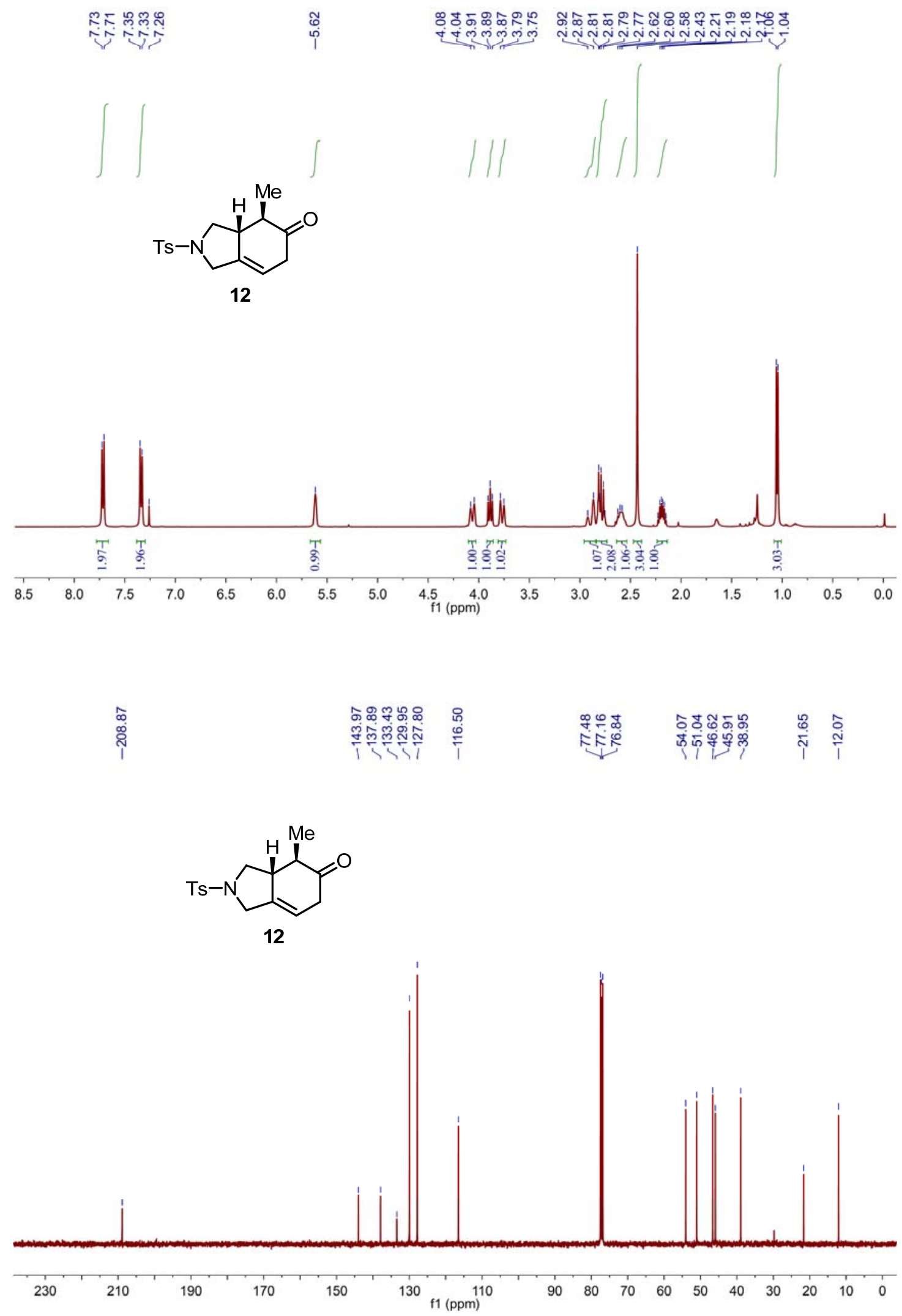



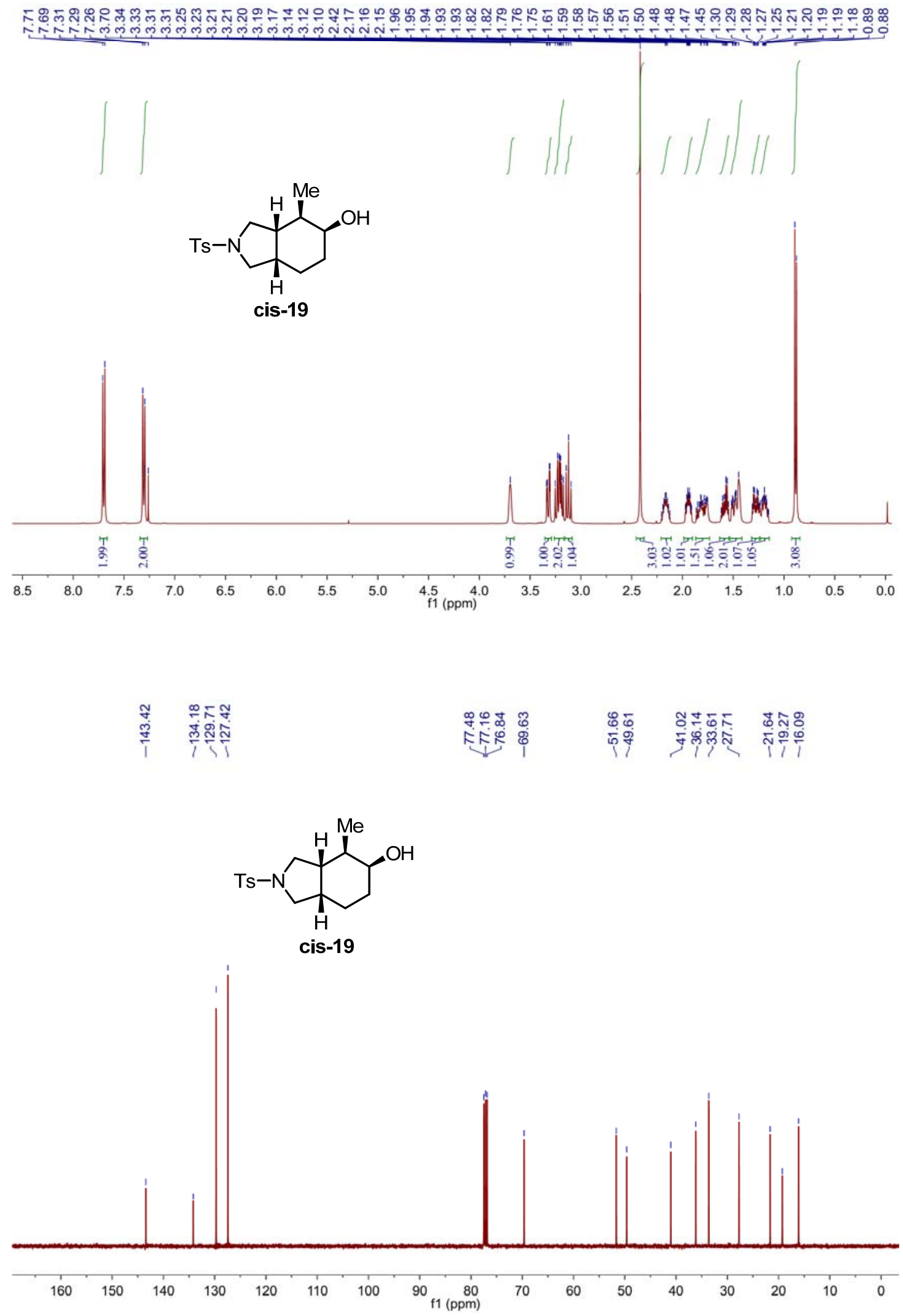